\title{
Nilálgebras comutativas de potências associativas
}

\author{
Mary Luz Rodiño Montoya
}

TESE APRESENTADA

$\mathrm{AO}$

INSTITUTO DE MATEMÁTICA E ESTATÍSTICA

DA

UNIVERSIDADE DE SÃO PAULO

PARA

OBTENÇÃO DO TÍTULO

$\mathrm{DE}$

DOUTOR EM CIENCIAS

Programa: Matemática.

Orientador: Prof. Dr. Juan Carlos Gutiérrez Fernández Co-Orientador: Prof. Dr. Alexandre Grichkov 


\title{
Nilálgebras comutativas de potências associativas
}

\author{
Este exemplar corresponde à redação \\ final da tese devidamente corrigida \\ e defendida por Mary Luz Rodiño Montoya \\ e aprovada pela Comissão Julgadora.
}

Banca Examinadora:

- Prof. Dr. Juan Carlos Gutiérrez Fernández (orientador) -IME-USP.

- Prof. Dr. Alexandre Grichkov (co-orientador) -IME-USP.

- Prof. Dr. Alexandre Ananin -UNICAMP.

- Prof. Dr. Plamen Emilov Koshlukov-UNICAMP.

- Prof. Dr. Aleksandr Pozhidaev -IM SB RAS. 
À minha Família, especialmente à minha Mãe. 


\section{Agradecimentos}

Agradeço ao professor Juan Carlos Gutiérrez Fernández, meu orientador, e ao professor Alexandre Grichkov, meu co-orientador, pela paciência, dedicação e pelos importantes aportes na elaboração desta tese. Também agradeço aos professores da banca de defesa pela valiosa contribuição com suas correções e sugestões, ao Instituto de Matemática e Estatística pela formação acadêmica e à possibilidade de utilização de seus recursos para meus estudos, à Cristina e ao Alexandre pela amizade e ajuda com o português na redação desta tese e a todos meus amigos pelo apoio e carinho. 


\section{Abstract}

The aim of this work is to study the structure of the modules over a trivial algebra of dimension two in the variety $\mathfrak{M}$ of commutative and powerassociative algebras. In particular we classify the irreducible modules. These results enables us to understand better the structure of finite-dimensional power-associative nilalgebras of nilindex 4 .

Finally, we classify, up to isomorphism, commutative power associative nilalgebras of nilindex $n$ and dimension $n$.

Keywords: Power associative algebras, nilalgebras, irreducible bimodules, power associative bimodules. 


\section{Resumo}

O objetivo deste trabalho é estudar a estrutura dos módulos sobre uma álgebra trivial de dimensão dois na variedade $\mathfrak{M}$ das álgebras comutativas de potências associativas. Em particular classificamos os módulos irredutíveis. Estes resultados nos permitem compreender melhor a estrutura das nilálgebras comutativas de dimensão finita e nilíndice 4.

Finalmente classificamos, sob isomorfismos, as nilálgebras comutativas de potências associativas de dimensão $n$ e nilíndice $n$.

Palavras-chave: Álgebras de potências associativas, nilálgebras, bimódulos irredutíveis, bimódulos de potências associativas. 


\section{Sumário}

$\begin{array}{ll}\text { Introdução } & 1\end{array}$

1 Preliminares $\quad 6$

2 Bimódulos de potências associativas $\quad 17$

2.1 Definição dos $\mathcal{A}$-submódulos $W_{\alpha}$. . . . . . . . . . . . . . . 19

$2.2 \mathcal{A}$-módulos irredutíveis. . . . . . . . . . . . . . . . . . . . 24

2.3 Decomposição de $M$ como soma dos $W_{\alpha} \ldots \ldots \ldots$

$3 \quad$ Nilálgebras de potências associativas e nilíndice $4 \quad 31$

3.1 O produto $W_{\alpha} \cdot W_{\beta}$ para $\alpha, \beta \in F^{*} \ldots \ldots . \ldots 33$

3.2 Os produtos $W_{\alpha}^{i} \cdot W_{\beta}^{j}$ para $\alpha, \beta \in F^{*} \ldots \ldots . \ldots 40$

3.2.1 Produtos para $i+j=3 \ldots \ldots \ldots . \ldots . \ldots 40$

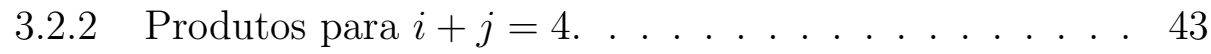

3.2.3 Produtos para $i+j=5$. . . . . . . . . . . . 49

3.2.4 Produtos para $i+j \geq 6$. . . . . . . . . 50

3.3 O produto $W_{0} \cdot W_{\alpha}$ para $\alpha \in F \ldots \ldots \ldots . \ldots . \ldots 5$

3.4 Algumas nilálgebras que não são simples. . . . . . . . . . . . 56

4 Dimensão e nilíndice $n$. $\quad 64$

4.1 Dimensão menor ou igual a quatro. . . . . . . . . . . . . 66 
4.2 Classificação para dimensão cinco. . . . . . . . . . . . . . . . 67

4.3 Classificação para dimensão seis. . . . . . . . . . . . . . . . . 87

4.4 Classificação para dimensão sete. . . . . . . . . . . . . . . . 110

4.5 Classificação para dimensão oito. . . . . . . . . . . . . . . . 130

4.6 Classificação para dimensão maior ou igual a nove. . . . . . . . 140

Referências Bibliográficas 165

$\begin{array}{lc}\text { Índice Remissivo } & 168\end{array}$ 


\section{Introdução}

As álgebras não-associativas são objeto de importantes estudos matemáticos. Paralelamente, elas têm despertado o interesse de pesquisadores de outras áreas, tais como a física teórica e a biomatemática. Por exemplo, as álgebras de Jordan foram introduzidas na mecânica quântica, as álgebras de Lie são utilizadas para descrever a estrutura local dos grupos topológicos e as álgebras báricas desempenham um papel central na teoria das álgebras genéticas. Focaremos nosso trabalho nas nilálgebras comutativas de potências associativas. Observe-se que as álgebras de Jordan são de potências associativas.

Em 1948, A. Albert levantou em [2] algumas questões, uma das quais sobre a existência de exemplos de nilálgebras simples de potências associativas. A seguinte conjectura ficou conhecida como o Problema de Albert: Toda nilálgebra comutativa de potências associativas e de dimensão finita é solúvel. Isto é equivalente a afirmar que, dentro da classe das nilálgebras comutativas de potências associativas, não existem álgebras simples de dimensão finita. Em 1972, D. Suttles [23] apresentou um exemplo de uma nilálgebra comutativa de potências associativas de dimensão cinco e nilíndice quatro que é solúvel mas não é nilpotente.

Exemplo de Suttles: Seja $\mathcal{B}$ uma álgebra comutativa com base $\left\{e_{1}, \ldots, e_{5}\right\}$ e produtos não nulos dados por

$$
e_{1} e_{2}=e_{2} e_{4}=-e_{1} e_{5}=e_{3}, \quad e_{1} e_{3}=e_{4}, \quad e_{2} e_{3}=e_{5} .
$$


Observamos que $\mathcal{B}^{2} \mathcal{B}^{2}=0$, logo $\mathcal{B}$ é solúvel de índice de solubilidade 2 , porém $\mathcal{B}^{2}=\mathcal{B}^{m} \neq 0$ para todo inteiro positivo $m$, o que mostra que $\mathcal{B}$ não é nilpotente. Assim, temos uma nilálgebra comutativa de potências associativas de nilíndice 4 que é solúvel mas não é nilpotente.

Deste modo, no problema de Albert, a condição de solubilidade não pode ser trocada pela condição de nilpotência.

M. Gerstenhaber em [13], num trabalho que faz parte de uma série de três artigos publicados entre 1958 e 1960, obteve o seguinte resultado sobre a estrutura das nilálgebras comutativas de potências associativas.

Teorema. (1) Seja $\mathcal{B}$ uma nilálgebra comutativa de potências associativas, sobre um corpo de característica zero ou de característica suficientemente grande se comparada ao indice de nilpotência $t$ de $b \in \mathcal{B}$. Então a álgebra gerada por $R_{b^{i}}$, com $i \in \mathbb{N}$, é nilpotente de indice menor ou igual a $2(t-1)^{2}+1$, sendo $R_{b}$ a multiplicação à direita de b por um elemento qualquer de $\mathcal{B}$. (2) $S e \mathcal{B}$ é uma nilálgebra comutativa de característica zero e nilíndice $t$, então $R_{b}^{2 t-3}=0$, para todo b em $\mathcal{B}$.

Em um trabalho de 1975 [12], M. Gerstenhaber e H. Myung realizaram um estudo das nilálgebras de dimensão pequena, mostrando a nilpotência da álgebra quando a dimensão é menor ou igual a quatro e determinando as classes de isomorfismos de tais álgebras.

Nos últimos dez anos, publicou-se uma série de artigos que forneceu uma solução parcial ao problema de Albert. Estes estudos podem ser classificados em duas linhas de pesquisa: uma delas aborda o problema por meio do estudo da dimensão da nilálgebra, no caso de dimensões pequenas, e a outra aborda o problema por meio do estudo do nilíndice quando ele é pequeno ou quando ele é grande com relação a dimensão. No caso do estudo da solubilidade (ou nilpotência) das nilálgebra de p.a. por meio de sua dimensão, foi mostrado por I. Correa e L. Peresi [7] que, para dimensão cinco, a álgebra é solúvel de índice menor ou igual a três, e que quando o nilíndice é três, a álgebra é nilpotente de índice de nilpotência menor ou igual a quatro. Para dimensão seis, I. Correa, I. Henzel e L. Peresi [6] mostraram a solubilidade da álgebra, 
e para dimensão sete, a solubilidade foi provada em [11] por L. Elgueta e A. Suazo. Neste mesmo trabalho foi mostrada a solubilidade da álgebra para dimensão oito para álgebra de nilíndice menor ou igual a quatro e, em [16], J.C. Gutiérrez Fernández e A. Suazo finalmente mostraram a solubilidade para nilíndice cinco ficando demonstrada a solubilidade para dimensão oito. Assim, podemos resumir todos estes resultados no seguinte teorema.

Teorema. Seja $\mathcal{B}$ uma nilálgebra comutativa de potências associativas, sobre um corpo de característica diferente de dois 2 e 3. Se $\mathcal{B}$ tem dimensão menor ou igual a 8, então $\mathcal{B}$ é solúvel.

Em função da nilpotência, quando o nilíndice é menor ou igual a três, o resultado é devido a Albert [21] já que, neste caso, a álgebra é de Jordan e, portanto, nilpotente se de dimensão finita. Para nilíndice $n$, sendo $n$ a dimensão da álgebra, I. Correa e A. Suazo [8] mostraram que a álgebra é nilpotente de índice de nilpotência $n$ (este resultado foi uma generalização do método utilizado por M. Gerstenhaber e H. Myung [12]). Eles encontraram também uma condição necessária e suficiente para que tais álgebras sejam de Jordan, e neste caso fizeram a classificação, sob a condição de que a característica não fosse um divisor de $n-2$. Para nilíndice maior ou igual a $n-2$, J.C. Gutiérrez Fernández [15] mostrou que a álgebra é solúvel, sendo neste caso a característica do corpo zero ou suficientemente grande se comparada ao nilíndice. Tais resultados podem ser condensados no seguinte teorema.

Teorema. Seja $\mathcal{B}$ uma nilálgebra comutativa de potências associativas, de dimensão finita e característica zero: (1) Se $\mathcal{B}$ tem nilíndice menor ou igual a 3, então $\mathcal{B}$ é nilpotente; (2) Se $\mathcal{B}$ tem dimensão n e nilíndice maior ou igual a $n-2$, então $\mathcal{B}$ é solúvel.

Além dos resultados anteriores, foram obtidos outros num contexto mais geral, como por exemplo o caso não comutativo, que foi considerado por I. Correa e I. Hentzel [4], os quais mostraram o seguinte: Se $\mathcal{B}$ é uma nilálgebra não comutativa de potências associativas de dimensão $n$ e nilíndice $n$ sobre um corpo de característica diferente de 2 , então $\mathcal{B}$ é solúvel e $\mathcal{B}^{2}$ é nilpo- 
tente. Para todo $n>2$, eles apresentaram dois exemplos de nilálgebras não comutativas de potências associativas de dimensão $n$. No primeiro exemplo, a álgebra tem nilíndice $n$ e não é nilpotente; no segundo, a álgebra tem nilíndice $n-1$ e não é solúvel.

J.C. Gutiérrez Fernández mostrou [15] que as nilálgebras comutativas (não necessariamente de potências associativas) de dimensão menor ou igual a 6 são solúveis se a característica do corpo for diferente de 2,3,5. Finalmente, I. Correa estabeleceu [3] que o índice de nilpotência de uma nilálgebra de Jordan de nilíndice $n-1$ e dimensão $n$ é $n-1$.

Considerando-se a profundidade e a complexidade do problema em questão, faz sentido continuar seu estudo em alguns casos particulares como tem sido feito até agora, para dimensões e nilíndices pequenos. Assim, na primeira parte desta tese (Capítulos 2 e 3), focamos nosso trabalho no estudo das nilálgebras de nilíndice 4. Mais explicitamente, consideremos $F$ um corpo algebricamente fechado de característica diferente de 2 e de 3 e $\mathfrak{M}$ a variedade das álgebras comutativas e de nilíndice 4 , sobre o corpo $F$. É claro que as $\mathfrak{M}$-álgebras são álgebras de potências associativas. Observamos que mesmo para as $\mathfrak{M}$-álgebras o problema de Albert está em aberto. É bom tentar aplicar métodos clássicos desenvolvidos para análise das álgebras de Lie, de Jordan, de Malcev, etc. para o estudo da estrutura das $\mathfrak{M}$-álgebras e para a construção de exemplos. O principal obstáculo que temos para o estudo da estrutura das $\mathfrak{M}$-álgebras é a ausência do análogo do Teorema de Engel na teoria das $\mathfrak{M}$-álgebras, que pode ser formulado da seguinte forma: Conjectura. Seja $\mathcal{B}$ uma $\mathfrak{M}$-álgebra de dimensão finita. Então $\mathcal{B}$ é nilpotente se, e somente se, para cada par de elementos $a, b \in \mathcal{B}$, tal que $a^{2}=b^{2}=a b=0$, a álgebra gerada pelos operadores $L_{a}$ e $L_{b}$ é nilpotente.

Esta conjectura mostra a importância do estudo dos módulos sobre a $\mathfrak{M}$-álgebra

$$
\mathcal{A}=\left\langle a, b \mid a^{2}=b^{2}=a b=0\right\rangle,
$$

isto é, a classificação dos $\mathcal{A}$-módulos na variedade $\mathfrak{M}$.

Desta maneira, no Capítulo 1, damos algumas definições básicas e resul- 
tados necessários para a compreensão do restante do trabalho. No Capítulo 2, desenvolvemos a teoria $\operatorname{dos} \mathcal{A}$-módulos $M$ de dimensão finita na classe $\mathfrak{M}$, caracterizando os irredutíveis. Definimos, para todo $\alpha \in F$, os $\mathcal{A}$-submódulos $W_{\alpha}$ e construímos uma base para tais módulos. Finalmente, demonstramos que $M$ pode ser decomposto como soma direta destes $W_{\alpha}$.

Uma vez que toda álgebra $\mathcal{B} \in \mathfrak{M}$ contém uma subálgebra trivial $\mathcal{A}$, é possível estudar a álgebra $\mathcal{B}$ por meio de sua estrutura de $\mathcal{A}$-módulo. Assim no Capítulo 3, estudamos a estrutura de $\mathcal{B}$ por meio de sua decomposição como soma direta dos $\mathcal{A}$-submódulos $W_{\alpha}$, com $\alpha \in F$. Estudaremos como são em $\mathcal{B}$ os produtos dos $\mathcal{A}$-submódulos $W_{\alpha}$.

Intentaremos nos aproximar do problema de Albert através dos módulos estudados no Capítulo 2. Isto nos permitirá construir alguns exemplos e buscar, se existir, algum contra-exemplo. Por exemplo, podemos analisar as álgebras em $\mathfrak{M}$ que são da forma $A+M$ onde $A$ é uma álgebra trivial de dimensão 2, $A M \subset M$ e $M$ é um $A$-módulo via $(x, m)=x m$ com $x \in A$ e $m \in M$.

Finalmente, no Capítulo 4, estudamos as nilálgebras comutativas de potências associativas de dimensão $n$ e nilíndice $n$ e damos uma classificação a menos de isomorfismo. 


\section{Capítulo 1}

\section{Preliminares}

Neste capítulo daremos alguns conceitos básicos das álgebras não-associativas, nilálgebras, álgebras de potências associativas e bimódulos. Alguns teoremas que serão utilizados nos outros capítulos serão apresentados sem sua demonstração, indicando sua respectiva referência.

Sejam $F$ um corpo, $\mathcal{B}$ um espaço vetorial sobre $F$ e

$$
\begin{aligned}
\mathcal{B} \times \mathcal{B} & \longrightarrow \mathcal{B} \\
(x, y) & \longmapsto x y
\end{aligned}
$$

uma aplicação bilinear tal que $\alpha(x y)=(\alpha x) y=x(\alpha y)$, para todo $x, y \in \mathcal{B}$ e $\alpha \in F$. O espaço vetorial $\mathcal{B}$, junto com a multiplicação definida através da aplicação bilinear, forma uma álgebra $\mathcal{B}$ sobre o corpo $F$. Se a multiplicação em $\mathcal{B}$ verifica que $x y=y x$, para todo $x, y \in \mathcal{B}$, então $\mathcal{B}$ chama-se comutativa e, se cumpre que $(x y) z=x(y z)$, para todo $x, y, z \in \mathcal{B}$, então $\mathcal{B}$ chama-se associativa. O termo álgebras não-associativas, será usado para fazer referência a álgebras não necessariamente associativas.

Seja $\Phi=\left\{e_{1}, \ldots, e_{n}\right\}$ uma base de uma álgebra $\mathcal{B}$ de dimensão finita. A multiplicação em $\mathcal{B}$ está determinada por $e_{i} e_{j}=\sum_{k=1}^{n} \gamma_{i j k} e_{k}$, com $1 \leq$ $i, j \leq n$. Os escalares $\gamma_{i j k} \in F$ são denominados constantes de estrutura da álgebra $\mathcal{B}$ com respeito à base dada $\Phi$. Se $V$ é um espaço vetorial sobre 
$F$, com base $\left\{e_{1}, \ldots, e_{n}\right\}$ e $\gamma_{i j k}(1 \leq i, j, k \leq n)$ são escalares, a expressão $e_{i} e_{j}=\sum_{k=1}^{n} \gamma_{i j k} e_{k}$ define uma multiplicação $x y=\sum_{k=1}^{n}\left(\sum_{i, j=1}^{n} \alpha_{i} \beta_{j} \gamma_{i j k}\right) e_{k}$ em que $x=\sum_{i=1}^{n} \alpha_{i} e_{i}$ e $y=\sum_{j=1}^{n} \beta_{j} e_{j}$ e munido desta multiplicação $V$ é uma álgebra sobre $F$. Observe que $V$ é comutativa se cumpre a identidade $\gamma_{i j k}=\gamma_{j i k}$, para todo $i, j, k$.

Sejam $\mathfrak{M}$ uma classe de álgebras sobre o corpo $F, A \in \mathfrak{M}, M$ é um espaço vetorial sobre $F$ e $A \times M \rightarrow M$ e $M \times A \rightarrow M$ duas aplicações bilineares dadas por $(a, m) \mapsto a m$ e $(m, a) \mapsto m a$. Então a soma direta $A \oplus M$ dos espaços vetoriais $A$ e $M$ é uma álgebra sobre $F$ com a multiplicação em $A \oplus M$ definida por:

$$
\left(a_{1}+m_{1}\right)\left(a_{2}+m_{2}\right):=a_{1} a_{2}+\left(m_{1} a_{2}+a_{1} m_{2}\right) .
$$

Se a álgebra $A \oplus M$ está em $\mathfrak{M}$, então $M$ é um bimódulo (no sentido de Eilenberg) sobre a álgebra $A$ (ou um $A$-bimódulo) na classe $\mathfrak{M}$.

Notemos que, se $\mathfrak{M}$ é a classe de todas as álgebras não-associativas sobre o corpo $F$, então nenhuma outra condição, fora da bilinearidade assumida originalmente será imposta às aplicações $A \times M \rightarrow M$ e $M \times A \rightarrow M$. Se a classe $\mathfrak{M}$ está definida por um conjunto de identidades multilineares $\left\{f_{i}\left(x_{1}, \ldots, x_{n_{i}}\right)=0 / i \in I\right\}$, então não é difícil ver que $M$ é um bimódulo para $A \in \mathfrak{M}$ na classe $\mathfrak{M}$ se e, somente se, as seguintes condições são satisfeitas:

$$
f_{i}\left(a_{1}, \ldots, a_{k-1}, m, a_{k+1}, \ldots, a_{n_{i}}\right)=0
$$

para todo $i \in I, k \in\left\{1, \ldots, n_{i}\right\}, a_{j} \in A, m \in M$.

Um subconjunto $N$ de $M$ é um $A$-subbimódulo de $M$ se ele mesmo é um bimódulo sobre $A$ na classe $\mathfrak{M}$. Um bimódulo $M$ é irredutível se não contém subbimódulos próprios, isto é seus únicos subbimódulos são 0 e $M$.

Um subespaço vetorial $S$ de uma álgebra $\mathcal{B}$, fechado sob a multiplicação, chama-se subálgebra de $\mathcal{B}$. Um subespaço $I$ de $\mathcal{B}$ fechado sob a multiplicação por elementos de $\mathcal{B}$, isto é, $x y, y x \in I$ para todo $x \in \mathcal{B}$ e $y \in I$, é um ideal de $\mathcal{B}$. Notemos que todo ideal é uma subálgebra, mas o contrário não é 
válido. A subálgebra $\mathcal{B}^{2}$, gerada como espaço vetorial pelos produtos $x y$ tal que $x, y \in \mathcal{B}$, é um ideal de $\mathcal{B}$. Uma álgebra $\mathcal{B}$ é simples quando $\mathcal{B}^{2} \neq 0$ e seus únicos ideais são os triviais, isto é, 0 e $\mathcal{B}$. Assim, se uma álgebra $\mathcal{B}$ é simples, então $\mathcal{B}=\mathcal{B}^{2}$. Seja $I$ um ideal de $\mathcal{B}$, o conjunto $\mathcal{B} / I=\{\bar{x}=x+I: x \in \mathcal{B}\}$ munido das operações $\bar{x}+\bar{y}=\overline{x+y}, \bar{x} \bar{y}=\overline{x y}$ e $\alpha \bar{x}=\overline{\alpha x}$, para todo $x, y \in \mathcal{B}$ e $\alpha \in F$ é a álgebra quociente de $\mathcal{B}$ por $I$. Observe que a álgebra $\mathcal{B} / \mathcal{B}^{2}$ tem multiplicação zero. Se $I, J$ são ideais de $\mathcal{B}$ tal que $I \cap J=0$ e sua soma é todo $\mathcal{B}$, então $\mathcal{B}$ é soma direta de $I$ e $J$ e é denotado por $\mathcal{B}=I \oplus J$. Note que para cada $x, y \in I$ e $z, w \in J$ temos que $(x+z)(y+w)=x y+z w$, pois $x w, z y \in$ $I \cap J=0$. O anulador de uma álgebra $\mathcal{B}$, que denotaremos por $\operatorname{Anul}(\mathcal{B})$, está formado pelos elementos de $\mathcal{B}$ cujo produto com qualquer outro elemento de $\mathcal{B}$ é nulo, isto é $\operatorname{Anul}(\mathcal{B})=\{x \in \mathcal{B} \mid x y=y x=0$, para todo $y \in \mathcal{B}\}$.

Uma transformação linear $h$ entre as $F$-álgebras $\mathcal{B}$ e $\mathcal{D}$, é um homomorfismo de álgebras, se $h(x y)=h(x) h(y)$, para todo $x, y \in \mathcal{B}$. É claro que o núcleo e a imagem de $h$, são subálgebras de $\mathcal{B}$ e $\mathcal{D}$ respectivamente, e que o núcleo é um ideal de $\mathcal{B}$. Se $I$ é um ideal de $\mathcal{B}$, então a inclusão $h: I \longrightarrow \mathcal{B}$, e a projeção $\pi: \mathcal{B} \longrightarrow \mathcal{B} / I$, são exemplos de homomorfismos de álgebras.

Se $x$ é um elemento de $\mathcal{B}$, então o operador linear $R_{x}: \mathcal{B} \longrightarrow \mathcal{B}$, definido por $R_{x}(y)=y x$, é denominado multiplicação à direita por $x$. De maneira análoga temos $L_{x}$, a multiplicação à esquerda por $x$, isto é, $L_{x}(y)=x y$ para todo $y \in \mathcal{B}$. Observemos que em geral, $R_{x}, L_{x}$ não são homomorfismos de álgebras. É claro que em álgebras comutativas, estes operadores coincidem, isto é $L_{x}=R_{x}$. Notemos que no caso de um $A$-bimódulo $M$ na classe $\mathfrak{M}$, as aplicações $\left.R_{x}\right|_{M}: m \mapsto m x$ e $\left.L_{x}\right|_{M}: m \mapsto x m$ para $x \in A$ e $m \in M$, são transformações lineares em $M$.

Uma transformação linear $f: \mathcal{B} \longrightarrow \mathcal{B}$ é nilpotente se existir um inteiro $k \geq 0$ tal que $f^{k} \equiv 0$. O menor $k$, cumprindo esta identidade, chama-se índice de nilpotência. Uma álgebra $\mathcal{B}$ chama-se de Engel se toda multiplicação à esquerda e à direita de $\mathcal{B}$ é nilpotente, isto é, os operadores $L_{x}$ e $R_{x}$ são nilpotentes para todo $x \in \mathcal{B}$. Uma álgebra $\mathcal{J}$ é de Jordan se é comutativa e satisfaz a seguinte lei de associatividade, chamada de identidade de Jordan, 
$x^{2}(y x)=\left(x^{2} y\right) x$, para todo $x, y$ em $\mathcal{J}$.

Até aqui, quase todos os conceitos apresentados são bem familiares, uma vez que eles são válidos em álgebras associativas. As principais diferenças entre as definições nas álgebras associativas e as não-associativas, estão naquelas que envolvem a multiplicação da álgebra. Assim uma das grandes diferenças está na definição das potências de uma álgebra e de um elemento, sendo que nas álgebras não associativas temos várias definições para as potências que coincidem quando a álgebra é associativa. Na continuação daremos algumas delas.

Seja $\mathcal{B}$ uma álgebra não-associativa. Se $C$ e $D$ são subespaços de $\mathcal{B}$, então $C D$ denota o subespaço vetorial gerado por todos os produtos $c d$, com $c \in C$ e $d \in D$. As seguintes potências são definidas por indução:

$$
\mathcal{B}^{1}=\mathcal{B}^{(0)}=\mathcal{B}
$$

e para $r \geq 2$ e $s \geq 0$

$$
\mathcal{B}^{r}=\sum_{i+j=r} \mathcal{B}^{i} \mathcal{B}^{j} \quad \text { e } \quad \mathcal{B}^{(s+1)}=\mathcal{B}^{(s)} \mathcal{B}^{(s)}
$$

Da definição anterior, é fácil ver por indução sobre $r$, para $r \geq 0$, que

$$
\left(\mathcal{B}^{(s)}\right)^{(r)}=\mathcal{B}^{(s+r)} .
$$

Em termos destas potências podem-se definir os seguintes conceitos: uma álgebra $\mathcal{B}$ é nilpotente se existir um inteiro $r$ tal que $\mathcal{B}^{r}=0$, e é solúvel se existir um inteiro $s$ tal que $\mathcal{B}^{(s)}=0$. Os menores $r$ e $s$ cumprindo estas propriedades chamam-se indice de nilpotência e indice de solubilidade, respectivamente. É fácil ver que uma álgebra $\mathcal{B}$ é nilpotente de índice $r$ se, e somente se, o produto de $r$ ou mais elementos com qualquer arranjo de parênteses é zero e existe um produto não nulo de $r-1$ elementos. Definimos também as potências de um elemento $x \in \mathcal{B}$, assim:

$$
x^{1}=x \quad e \quad x^{i+1}=x^{i} x \quad \text { para } \quad i=1,2, \ldots
$$


Notemos que toda álgebra nilpotente é solúvel uma vez que $\mathcal{B}^{(r)} \subseteq \mathcal{B}^{2^{r}}$. Podemos verificar a inclusão anterior por indução sobre $r$ assim: Se $r=0$, $\mathcal{B}^{(0)} \subseteq \mathcal{B}^{2^{0}}$, e supondo para valores menores ou iguais a $r-1$, temos para $r$ que $\mathcal{B}^{(r)}=\mathcal{B}^{(r-1)} \mathcal{B}^{(r-1)} \subseteq \mathcal{B}^{2^{r-1}} \mathcal{B}^{2^{r-1}} \subseteq \mathcal{B}^{2^{r}}$, cumprindo-se a inclusão. Por outro lado, temos que a solubilidade não implica a nilpotência, basta tomar a álgebra $\mathcal{B}$ gerada pelo conjunto $\left\{e_{1}, e_{2}\right\}$, onde $e_{1}^{2}=e_{2}^{2}=0$ e $e_{1} e_{2}=-e_{2} e_{1}=$ $e_{1}$. Como $\mathcal{B}^{2}=\left\langle e_{1}\right\rangle, \mathcal{B}^{2} \mathcal{B}^{2}=0$ e $\mathcal{B}^{(2)}=\mathcal{B}^{(1)} \mathcal{B}^{(1)}=\mathcal{B}^{2} \mathcal{B}^{2}=0$. Assim, $\mathcal{B}$ é solúvel de índice 2 , mas $\mathcal{B B}^{2}=\mathcal{B}^{2}, \operatorname{logo} \mathcal{B}^{2}=\mathcal{B}^{3}=\ldots=\mathcal{B}^{r}=\ldots \neq 0$. Portanto, $\mathcal{B}$ não é nilpotente.

Se $\mathcal{B}$ é uma álgebra de dimensão finita, então $\mathcal{B}$ contém um único ideal maximal solúvel $S$. Portanto, a álgebra quociente $\mathcal{B} / S$ não contém ideais solúveis não nulos. O ideal $S$ chama-se o radical solúvel da álgebra $\mathcal{B}$ e é denotado por $\operatorname{Sol}(\mathcal{B})$.

Um elemento $x$ em $\mathcal{B}$ é nilpotente se a subálgebra gerada por ele é nilpotente. A partir deste conceito podemos introduzir a definição de nilálgebra, que é uma das principais características das álgebras que vamos estudar nesta tese. Uma álgebra $\mathcal{B}$ é uma nilálgebra se todos seus elementos são nilpotentes. Assim, se $\mathcal{B}$ é uma nilálgebra, então para cada $x \in \mathcal{B}$, existe um inteiro $k$ tal que o produto de $x, k$ ou mais vezes, com qualquer associação, é zero. Se $k$ pode ser escolhido independente de $x$, então diz-se que $\mathcal{B}$ tem nilíndice $k$, sendo $k$ o menor inteiro satisfazendo esta propriedade.

Introduziremos na continuação a noção de álgebras de potências associativas, assim como alguns teoremas a respeito desta classe de álgebras.

Uma álgebra $\mathcal{B}$ é de potências associativas, (p.a.), se para todo $x \in \mathcal{B}$, a subálgebra gerada por $x$ é associativa, assim definidas as potências dos elementos $x$ de $\mathcal{B}$ por

$$
x^{1}=x \quad e \quad x^{i+1}=x^{i} x \quad \text { para } \quad i=1,2, \ldots
$$


temos que $\mathcal{B}$ é de potências associativas, se para todo $x \in \mathcal{A}$

$$
x^{i} x^{j}=x^{i+j} \quad \text { para } \quad i, j=1,2, \ldots
$$

Numa álgebra de p.a. temos que

$$
\left(x^{r}\right)^{s}=x^{r s}, \quad x^{r} x^{s}=x^{r+s} .
$$

Assim definidas as potências de um elemento, temos que numa álgebra de p.a. um elemento $x$ é nilpotente se $x^{k}=0$, para algum $k$.

Se $\mathcal{B}$ é uma álgebra comutativa de p.a. de dimensão finita, então $\mathcal{B}$ contém um único nilideal maximal $\operatorname{Nil}(\mathcal{B})$, chamado de nilradical da álgebra $\mathcal{B}$. Em uma álgebra $\mathcal{B}$ de p.a. de dimensão finita, tem-se que $S(\mathcal{B}) \subseteq \operatorname{Nil}(\mathcal{B})$, isto é, o radical solúvel de $\mathcal{B}$ está contido no nilradical de $\mathcal{B}$.

Assim neste contexto, o Problema de Albert pode ser reformulado em termos de radicais: na classe das álgebras comutativas de potências associativas de dimensão finita o radical solúvel é igual ao nilradical.

Seja $\mathcal{B}$ uma álgebra comutativa de p.a. sobre um corpo de característica diferente de 2 e 3 . Então linearizando a identidade (ver teoria de linearização em K. Zevlakov [25], pag. 14)

$$
x^{4}=x^{2} x^{2}
$$

obtemos as seguintes novas identidades:

$$
\begin{gathered}
2 x(x(x y))+x\left(x^{2} y\right)+x^{3} y=4 x^{2}(x y), \\
2 y(x(x y))+2 x(y(x y))+x\left(x y^{2}\right)+y\left(x^{2} y\right)=4(x y)^{2}+2 x^{2} y^{2}, \\
2 x(x(y z))+2 x(z(x y))+2 z(x(x y))+2 x(y(x z)) \\
+z\left(x^{2} y\right)+2 y(x(x z))+y\left(x^{2} z\right)=4 x^{2}(y z)+8(x y)(x z),
\end{gathered}
$$

para quaisquer elementos $x, y, z$ em $\mathcal{B}$. 
O seguinte resultado, que caracteriza a álgebra gerada pelos operadores $L_{x^{i}}$, foi apresentado por Albert em [2].

Lema 1.1 Seja $\mathcal{B}$ uma álgebra comutativa de p.a. sobre um corpo de característica diferente de 2 e 3 . Então, para cada $x \in \mathcal{B}$, a álgebra gerada pelos operadores $L_{x^{i}}$ com $i \geq 1$, é gerada por $L_{x}$ e $L_{x^{2}}$.

Prova: Trocando $z$ por $x^{r}$ em (1.3) temos que

$$
\begin{aligned}
2 x\left(x\left(x^{r} y\right)\right)+2 x( & \left.x^{r}(x y)\right)+2 x^{r}(x(x y))+2 x\left(x^{r+1} y\right) \\
+x^{r}\left(x^{2} y\right)+3 x^{r+2} y & =4 x^{2}\left(x^{r} y\right)+8 x^{r+1}(x y),
\end{aligned}
$$

ou equivalentemente

$$
\begin{aligned}
3 L_{x^{r+2}}= & 4 L_{x^{2}} L_{x^{r}}+8 L_{x^{r+1}} L_{x}-2 L_{x}^{2} L_{x^{r}}-2 L_{x} L_{x^{r}} L_{x} \\
& -2 L_{x} L_{x^{r+1}}-2 L_{x^{r}} L_{x}^{2}-L_{x^{r}} L_{x^{2}} .
\end{aligned}
$$

De (1.4) obtemos por indução sobre $r$, que $L_{x^{r+2}} \in\left\langle L_{x}, L_{x^{2}}\right\rangle$ para $r \geq 1$.

Utilizando indução e as identidades (1.1), (1.3) e (1.4) é possível demonstrar o seguinte teorema. Para mais detalhes veja [2].

Teorema 1.2 . Seja $\mathcal{B}$ uma álgebra comutativa sobre um corpo $F$ de característica diferente de 2,3 e 5 . Então $\mathcal{B}$ é de p.a. se, e somente se, $x^{4}=x^{2} x^{2}$, para todo $x \in \mathcal{B}$.

Em [1], Albert mostrou que toda álgebra de característica zero ou prima com 30 que satisfaz as identidades $x x^{2}=x^{2} x$ e $x^{2} x^{2}=\left(x^{2} x\right) x$ é de potências associativas. Neste caso a álgebra não é necessariamente comutativa. É importante ressaltar que a restrição na característica é necessária. Três exemplos de álgebras comutativas que cumprem a identidade $x^{2} x^{2}=x^{4}$ e não são de potências associativas podem ser vistos em [1].

O seguinte resultado para espaços vetoriais foi apresentado por Gerstenhaber em [14]. Uma outra demonstração do lema foi dada por Mathes, Omladic e Radjavi em [19]. 
Lema 1.3 Seja $V$ um espaço vetorial de dimensão n sobre um corpo com pelo menos $n$ elementos, e $H$ um subespaço vetorial de endomorfismos nilpotentes de $V . \operatorname{Então} \operatorname{dim}(H) \leq n(n-1) / 2$, e se $\operatorname{dim}(H)=n(n-1) / 2$, então o espaço vetorial $H$ é uma álgebra nilpotente de índice $n$.

Um outro resultado, relacionado com o anterior, foi dado por Gutiérrez Fernández em [15].

Lema 1.4 Sejam $V$ um espaço vetorial de dimensão 3 sobre um corpo $F$ com pelo menos 3 elementos. Se $H$ é um subespaço vetorial de endomorfismos nilpotentes de End $(V)$, então verifica-se uma das seguintes possibilidades:

(i) Existe um elemento não nulo $v \in V$ no núcleo de todos os $h \in H$.

(ii) $\operatorname{dim}(H)=2$. Para todo $0 \neq h \in H$, tem-se posto $(h)=2$. Se $H=$ $\langle f, g\rangle$, então existe $\left\{v_{1}, v_{2}, v_{3}\right\}$ base de $V$ tal que: $f\left(v_{1}\right)=v_{2}, f\left(v_{2}\right)=$ $v_{3}, f\left(v_{3}\right)=0$ e $g\left(v_{1}\right)=0, g\left(v_{2}\right)=\beta v_{1}, g\left(v_{3}\right)=-\beta v_{2}$, com $\beta \neq 0$.

Prova: Seja $H$ um espaço vetorial de aplicações lineares nilpotentes sobre um espaço vetorial $V$ de dimensão 3, que não satisfaz a condição (i). Pelo Lema $1.3, \operatorname{dim}(H)=2$. Sejam $f, g$ tais que $H=\langle f, g\rangle$. Como $f, g$ são nilpotentes, $\operatorname{ker}(f) \neq\{0\}$ e $\operatorname{ker}(g) \neq\{0\}$; por outro lado, $\operatorname{dim}(V)=3=$ $\operatorname{dim}(\operatorname{ker}(f))+\operatorname{dim}(i m(f))$ (igualmente para $g$ ), logo $\operatorname{dim}(k e r(f))=1$ ou $\operatorname{dim}(\operatorname{ker}(g))=1$, portanto, posto $(f)=2$ ou posto $(g)=2$.

Suponhamos que posto $(f)=2$. Usando a forma canônica de Jordan de $f$, o endomorfismo $f$ pode ser representado, relativo a uma base adequada $\Phi=\left\{v_{1}, v_{2}, v_{3}\right\}$ de $V$ como $f\left(x_{1}, x_{2}, x_{3}\right)=\left(x_{2}, x_{3}, 0\right)$, isto é,

$$
\mathbf{A}=\left(\begin{array}{lll}
0 & 1 & 0 \\
0 & 0 & 1 \\
0 & 0 & 0
\end{array}\right)
$$

é a matriz de $f$ com respeito à base $\Phi$, e então obviamente $v_{1} \in \operatorname{ker}(f)$. A representação anterior de $f$ é invariante a respeito de bases da forma 
$\left\{\gamma v_{1}, \beta v_{1}+\gamma v_{2}, \alpha v_{1}+\beta v_{2}+\gamma v_{3}\right\}, \operatorname{com} \alpha, \beta, \gamma \in F$ e $\gamma \neq 0$. Seja $B=\left[\beta_{i j}\right]$ a matriz de $g$ com respeito à base $\Phi$. Para todo $\lambda \in F, \lambda f+g \in H$, e portanto $\lambda f+g: V \longrightarrow V$ é nilpotente, e uma vez que $\operatorname{dim}(V)=3$, temos que seu polinômio característico é $P(\lambda f+g):=t^{3}$. Por outro lado, $P(\lambda f+g)=\operatorname{det}(t I-\lambda A-B)=t^{3}-s_{2} t^{2}+s_{1} t+s_{0}$, onde

$$
\begin{aligned}
& s_{2}=\beta_{11}+\beta_{22}+\beta_{33}, \\
& s_{1}=\left|\begin{array}{cc}
\beta_{11} & \lambda+\beta_{12} \\
\beta_{21} & \beta_{22}
\end{array}\right|+\left|\begin{array}{cc}
\beta_{11} & \beta_{13} \\
\beta_{31} & \beta_{33}
\end{array}\right|+\left|\begin{array}{cc}
\beta_{22} & \lambda+\beta_{23} \\
\beta_{32} & \beta_{33}
\end{array}\right|, \\
& s_{0}=\left|\begin{array}{ccc}
\beta_{11} & \lambda+\beta_{12} & \beta_{13} \\
\beta_{21} & \beta_{22} & \lambda+\beta_{23} \\
\beta_{31} & \beta_{32} & \beta_{33}
\end{array}\right| .
\end{aligned}
$$

Para $i=0,1,2$, podemos exprimir $s_{i}$ como polinômios em $\lambda$ da seguinte maneira

$$
s_{i}=s_{i 2} \lambda^{2}+s_{i 1} \lambda+s_{i 0}
$$

onde

$$
\begin{aligned}
& s_{22}=s_{21}=0, \quad s_{20}=\beta_{11}+\beta_{22}+\beta_{33}, \quad s_{12}=0, \\
& s_{11}=\beta_{32}+\beta_{21}, \quad s_{10}=\left|\begin{array}{ll}
\beta_{11} & \beta_{12} \\
\beta_{21} & \beta_{22}
\end{array}\right|+\left|\begin{array}{ll}
\beta_{11} & \beta_{13} \\
\beta_{31} & \beta_{33}
\end{array}\right|+\left|\begin{array}{cc}
\beta_{22} & \beta_{23} \\
\beta_{32} & \beta_{33}
\end{array}\right|, \\
& s_{02}=\beta_{31}, \quad s_{01}=\beta_{32} \beta_{11}+\beta_{21} \beta_{33}-\beta_{31} \beta_{12}-\beta_{23} \beta_{31}, \\
& s_{00}=-\beta_{33}\left|\begin{array}{ll}
\beta_{11} & \beta_{12} \\
\beta_{21} & \beta_{22}
\end{array}\right|+\beta_{23}\left|\begin{array}{cc}
\beta_{11} & \beta_{12} \\
\beta_{31} & \beta_{32}
\end{array}\right|-\beta_{13}\left|\begin{array}{cc}
\beta_{21} & \beta_{22} \\
\beta_{31} & \beta_{32}
\end{array}\right| .
\end{aligned}
$$

Agora, percorrendo $\lambda$ pelo menos três elementos diferentes de $F$ obtemos que 
$s_{i j}=0$, para todo $i, j \in\{0,1,2\}$. Em particular, de $s_{02}=s_{11}=s_{20}=0$ temos que

$$
\beta_{31}=0, \quad \beta_{21}+\beta_{32}=0, \quad \beta_{11}+\beta_{22}+\beta_{33}=0 .
$$

A seguir, a prova será dividida em dois casos.

- Caso 1: Se $k e r(g) \nsubseteq\left\langle v_{1}, v_{2}\right\rangle$, podemos assumir que $v_{3} \in k e r(g)$. Assim, de (1.5) temos que a matriz $B$ tem a forma

$$
\mathbf{B}=\left(\begin{array}{ccc}
\beta_{11} & \beta_{12} & 0 \\
\beta_{21} & -\beta_{11} & 0 \\
0 & -\beta_{21} & 0
\end{array}\right) .
$$

Dado que, $s_{01}=0$ e $\beta_{33}=\beta_{31}=0$, segue que $\beta_{32} \beta_{11}=0$, isto é,

$$
\beta_{21} \beta_{11}=0 \text {. }
$$

Por outro lado temos que $s_{10}=0$ e $\beta_{13}=\beta_{23}=\beta_{33}=0$, logo $\beta_{11} \beta_{22}-$ $\beta_{12} \beta_{21}=0$, isto é,

$$
\beta_{11}^{2}+\beta_{12} \beta_{21}=0 \text {. }
$$

De (1.6) e (1.7), e usando a hipótese de $v_{1} \notin k e r(g)$, obtemos que $\beta_{11}=\beta_{12}=0$ e $\beta_{21} \neq 0$. Assim, chamando $\beta_{21}$ de $\beta$, temos que $g$ está representado com respeito à base $\Phi$ como $g\left(x_{1}, x_{2}, x_{3}\right)=\beta\left(0, x_{1},-x_{2}\right)$, $\operatorname{com} \beta \neq 0$.

- Caso 2: Se $k e r(g) \subseteq\left\langle v_{1}, v_{2}\right\rangle$, então como $v_{1} \notin k e r(g)$, podemos assumir $v_{2} \in \operatorname{ker}(g), \log 0 \beta_{32}=0$ e por (1.5) temos que $\beta_{21}=0$. Portanto a matriz $B$ tem a seguinte forma

$$
\mathbf{B}=\left(\begin{array}{ccc}
\beta_{11} & 0 & \beta_{13} \\
0 & 0 & \beta_{23} \\
0 & 0 & -\beta_{11}
\end{array}\right)
$$

Como $B$ é nilpotente e triangular superior $\beta_{11}=0$, e isto implica que $v_{1} \in \operatorname{ker}(g)$, contradizendo a hipótese. Portanto, este caso não é possível. 
Como mostramos que posto $(f)=\operatorname{posto}(g)=2$, podemos repetir o processo utilizando primeiro o endomorfismo $g$ e mostrando que $f, g$ podem ser representadas como $f\left(x_{1}, x_{2}, x_{3}\right)=\gamma\left(0, x_{1},-x_{2}\right)$ com $\gamma \neq 0$ e $g\left(x_{1}, x_{2}, x_{3}\right)=\left(x_{2}, x_{3}, 0\right)$, para uma base adequada de $V$. 


\section{Bimódulos de potências} associativas

Neste capítulo desenvolveremos a teoria dos bimódulos de potências associativas, sobre uma álgebra de multiplicação zero. Para tal, seja $F$ um corpo algebricamente fechado de característica diferente de 2 e 3 e seja $\mathfrak{M}$ a variedade das álgebras comutativas de potências associativas. Seja $\mathcal{A} \in \mathfrak{M}$ uma álgebra de multiplicação zero e dimensão 2, isto é

$$
\mathcal{A}=\left\langle a, b \mid a^{2}=b^{2}=a b=0\right\rangle
$$

e $M$ um $\mathcal{A}$-bimódulo, na classe $\mathfrak{M}$. Notemos que nesta classe, os conceitos de módulo e bimódulo coincidem, pois as álgebras são comutativas, portanto de agora em diante nos referiremos a $M$ simplesmente como um $\mathcal{A}$-módulo e a $\mathcal{A}$ como a álgebra trivial de dimensão dois definida acima. Ao longo deste capítulo, $F, \mathfrak{M}, \mathcal{A}$ e $M$ são fixos e $n=\operatorname{dim} M$.

Neste capítulo, caracterizaremos os $\mathcal{A}$-módulos irredutíveis em $\mathfrak{M}$, definiremos os $\mathcal{A}$-submódulos $W_{\alpha}$ para cada $\alpha \in F$, construiremos uma base para tais $\mathcal{A}$-submódulos e demonstraremos finalmente que todo $\mathcal{A}$-módulo $M$, de dimensão finita, se decompõe como soma direta dos $W_{\alpha}$, com $\alpha \in F$.

Para começar este estudo, seja $x \in \mathcal{A}$ e $L_{x}$ a restrição em $M$ da transformação linear multiplicação à esquerda na álgebra $\mathcal{A} \oplus M$, isto é, $L_{x}$ : $M \longrightarrow M$ tal que $m \mapsto x m$ para todo $m \in M$. Como estamos trabalhando 
com álgebras comutativas, as transformações multiplicação à esquerda e multiplicação à direita coincidem, portanto utilizaremos apenas $L_{x}$.

Sabemos que todos os elementos das álgebras em $\mathfrak{M}$, satisfazem as identidades

$$
x y=y x \quad \text { e } \quad x^{4}=x^{2} x^{2} .
$$

Se linearizamos a segunda identidade, obtemos

$$
2 x(x(x y))+x\left(x^{2} y\right)+x^{3} y=4 x^{2}(x y),
$$

que em termos do operador $L_{x}$ é equivalente a

$$
2 L_{x}^{3}+L_{x} L_{x^{2}}+L_{x^{3}}=4 L_{x^{2}} L_{x} .
$$

Uma vez que $\mathcal{A}^{2}=0$, temos de $(2.1)$ que $x(x(x m))=0$ ou $L_{x}^{3}=0$, para todo $x \in \mathcal{A}$ e todo $m \in M$, e linearizando esta identidade obtemos:

$$
x(x(y m))+x(y(x m))+y(x(x m))=0,
$$

para todo $x, y \in \mathcal{A}$, isto é,

$$
L_{x}^{2} L_{y}+L_{x} L_{y} L_{x}+L_{y} L_{x}^{2}=0
$$

Em particular, lembrando que temos $a, b \in \mathcal{A}$ fixos,

$$
L_{a}^{3}=L_{b}^{3}=0, \quad L_{a}^{2} L_{b}+L_{a} L_{b} L_{a}+L_{b} L_{a}^{2}=0 \quad \text { e } \quad L_{b}^{2} L_{a}+L_{b} L_{a} L_{b}+L_{a} L_{b}^{2}=0 .
$$

Definimos agora

$$
V:=\operatorname{ker} L_{a} .
$$

Observamos que $V \neq 0$, pois $L_{a}^{3}=0$, e como para todo $v \in V$,

$$
L_{a}^{2} L_{b}(v)=-L_{a} L_{b} L_{a}(v)-L_{b} L_{a}^{2}(v)=0,
$$

segue que $V$ é invariante pelo operador $L_{a} L_{b}$. Portanto, podemos definir a seguinte transformação linear em $V$

$$
\begin{aligned}
\varphi: V & \longrightarrow V \\
v & \mapsto a b v
\end{aligned}
$$


isto é $\varphi=L_{a} L_{b}$ restrita a $V$. Para simplificar a notação, se $x_{1}, \ldots, x_{k} \in \mathcal{A}$ e $v \in V$, então $\mathrm{m} x_{1} \ldots x_{k} v$ representa o produto principal $x_{1}\left(x_{2}\left(\ldots\left(x_{k} v\right) \ldots\right)\right.$ ).

Para cada $\alpha \in F$ definimos

$$
V_{\alpha}:=\left\{v \in V \mid(\varphi-\alpha I)^{n}(v)=0\right\}
$$

e para $k=0, \ldots, n$, definimos

$$
V_{\alpha, k}:=\operatorname{ker}(\varphi-\alpha I)^{k}
$$

É claro da definição que $V_{\alpha, n}=V_{\alpha} \quad$ e

$$
0=V_{\alpha, 0} \subset V_{\alpha, 1} \subset \cdots \subset V_{\alpha, n}=V_{\alpha}
$$

Temos também que $v_{k} \in V_{\alpha, k}$, se, e somente se,

$$
\varphi\left(v_{k}\right)=\alpha v_{k}+v_{k-1},
$$

com $v_{k-1} \in V_{\alpha, k-1}$, para $k \geq 1$.

Notemos que $V_{\alpha}=0$ se, e somente se, $\alpha$ não é autovalor de $\varphi$. Como $F$ é algebricamente fechado, temos pelo Teorema de Decomposição Primária para endomorfismos sobre um espaço de dimensão finita que

$$
V=\bigoplus_{\alpha \in F} V_{\alpha}=\bigoplus_{\alpha \in \Delta} V_{\alpha}
$$

onde $\Delta$ é o conjunto finito formado por todos os autovalores de $\varphi$.

Para todo inteiro não negativo $k$, temos que $V_{\alpha, k}$ é invariante por $\varphi$ e se $\alpha \neq 0$, então $\varphi$ restrita a $V_{\alpha, k}$ é injetora para todo $k \geq 1$.

\subsection{Definição dos $\mathcal{A}$-submódulos $W_{\alpha}$}

Agora que conhecemos algumas propriedades dos espaços vetoriais $V_{\alpha, k}$, estamos em condição de definir os $\mathcal{A}$-submódulos $W_{\alpha}$, para cada $\alpha \in F$. Esta 
definição será feita discriminando os casos $\alpha \neq 0$ e $\alpha=0$. Denotaremos por $F^{*}$ o conjunto dos escalares em $F$ diferentes de zero. Se $\alpha \in F^{*}$, então

$$
W_{\alpha}:=V_{\alpha}+b V_{\alpha}+b b V_{\alpha}
$$

e para $k=0, \ldots, n$, definimos

$$
W_{\alpha, k}:=V_{\alpha, k}+b V_{\alpha, k}+b b V_{\alpha, k} .
$$

Da definição segue imediatamente que $W_{\alpha, n}=W_{\alpha}$ e que

$$
0=W_{\alpha, 0} \subset W_{\alpha, 1} \subset \cdots \subset W_{\alpha, n}=W_{\alpha} .
$$

Notemos que $W_{\alpha, k}$ é invariante pela multiplicação por $a$ e $b$, pois $L_{a}\left(V_{\alpha, k}\right)=$ $0, L_{a}\left(b V_{\alpha, k}\right)=\varphi\left(V_{\alpha, k}\right) \subset V_{\alpha, k}$ e $L_{a}\left(b b V_{\alpha, k}\right)=L_{a} L_{b}^{2}\left(V_{\alpha, k}\right) \subset-L_{b}^{2} L_{a}\left(V_{\alpha, k}\right)-$ $L_{b} L_{a} L_{b}\left(V_{\alpha, k}\right)=-b \varphi\left(V_{\alpha, k}\right) \subset b V_{\alpha, k}$, isto é $L_{a}\left(W_{\alpha, k}\right) \subset W_{\alpha, k}$ e claramente $L_{b}\left(W_{\alpha, k}\right) \subset W_{\alpha, k}$. Logo $W_{\alpha, k}$ é um $\mathcal{A}$-submódulo de $M$, para $k=0, \ldots, n$.

Definimos assim, para $\alpha \in F^{*}$, os $\mathcal{A}$-submódulos $W_{\alpha}$ e, para todo $k \geq 1$, os $\mathcal{A}$-submódulos $W_{\alpha, k}$. Queremos agora construir uma base para os $\mathcal{A}$ submódulos $W_{\alpha, k}$, e portanto para $W_{\alpha}=W_{\alpha, n}$. Dado que definimos $W_{\alpha}$ a partir dos espaços vetoriais $V_{\alpha}$, para os quais temos conhecidas e importantes propriedades, queremos definir una base para $W_{\alpha}$ a partir de uma base conhecida de $V_{\alpha}$, o que de fato é possível, como demonstraremos no seguinte teorema. Antes de passar ao teorema, notemos que dada uma base para $V_{\alpha, k-1}$, é sempre possível construir uma base para $V_{\alpha, k}$ contendo a base de $V_{\alpha, k-1}$, para todo $k \geq 2$.

Teorema 2.1 Sejam $\alpha \in F^{*}, k \geq 1$ e $\left\{u_{1}, u_{2}, \ldots, u_{t_{k}}\right\}$ uma base para $V_{\alpha, k}$. Então o conjunto $\left\{u_{1}, b u_{1}, b b u_{1}, u_{2}, b u_{2}, b b u_{2}, \ldots, u_{t_{k}}, b u_{t_{k}}, b b u_{t_{k}}\right\}$ é uma base para $W_{\alpha, k}$.

Prova: Uma vez que $W_{\alpha, k}=V_{\alpha, k}+b V_{\alpha, k}+b b V_{\alpha, k}$, é claro que se $\left\{u_{1}, u_{2}, \ldots, u_{t_{k}}\right\}$, é uma base para $V_{\alpha, k}$, então o conjunto

$$
\left\{u_{1}, b u_{1}, b b u_{1}, u_{2}, b u_{2}, b b u_{2}, \ldots, u_{t_{k}}, b u_{t_{k}}, b b u_{t_{k}}\right\}
$$


é um conjunto gerador para $W_{\alpha, k}$. Portanto resta apenas mostrar que este conjunto é linearmente independente e portanto uma base para $W_{\alpha, k}$.

Mostraremos primeiro que, se o conjunto $\left\{u_{1}, u_{2}, \ldots, u_{t}\right\} \subset V_{\alpha, k}$ é linearmente independente, então o conjunto $\left\{u_{1}, b u_{1},, u_{2}, b u_{2}, \ldots, u_{t}, b u_{t}\right\}$ é linearmente independente. Sejam $\beta_{i}, \lambda_{i} \in F$ tal que

$$
\sum_{i=1}^{t} \beta_{i} u_{i}+\lambda_{i} b u_{i}=0 .
$$

Então multiplicando por $a$,

$$
\sum_{i=1}^{t} \beta_{i} a u_{i}+\lambda_{i} a b u_{i}=\sum_{i=1}^{t} \varphi\left(\lambda_{i} u_{i}\right)=\varphi\left(\sum_{i=1}^{t} \lambda_{i} u_{i}\right)=0,
$$

pois $a u_{i}=0$, para todo $i$, e como $\sum_{i=1}^{t} \lambda_{i} u_{i} \in V_{\alpha, k}$ e $\left.\varphi\right|_{V_{\alpha, k}}$ é injetora, para $\alpha \in F^{*}$, segue que $\sum_{i=1}^{t} \lambda_{i} u_{i}=0$. E portanto $\lambda_{i}=0$ para $i=1, \ldots, t$, logo $\sum_{i=1}^{t} \beta_{i} u_{i}=0$, de onde $\beta_{i}=0$, para $i=1, \ldots, t$.

Vejamos agora que, se o conjunto $\left\{u_{1}, u_{2}, \ldots, u_{t}\right\} \subset V_{\alpha, k}$ é linearmente independente, então o conjunto $\left\{u_{1}, b u_{1}, b b u_{1}, u_{2}, b u_{2}, b b u_{2}, \ldots, u_{t}, b u_{t}, b b u_{t}\right\}$ é linearmente independente. Para isto, sejam $\beta_{i}, \lambda_{i}, \rho_{i} \in F$ tal que

$$
\sum_{i=1}^{t} \beta_{i} u_{i}+\lambda_{i} b u_{i}+\rho_{i} b b u_{i}=0
$$

Então, multiplicando duas vezes por $a$, temos que

$$
\sum_{i=1}^{t} \beta_{i} a a u_{i}+\lambda_{i} a a b u_{i}+\rho_{i} a a b b u_{i}=0
$$

$\operatorname{mas} a a u_{i}=0, a a b u_{i}=-a b a u_{i}-b a a u_{i}=0$ e $a a b b u_{i}=-a b a b u_{i}-a b b a u_{i}=$ $-a b a b u_{i}$, pois $u_{i} \in V_{\alpha, k}, \log \mathrm{O}$

$$
\sum_{i=1}^{t} \rho_{i} a a b b u_{i}=-\sum_{i=1}^{t} \rho_{i} a b a b u_{i}=-\varphi^{2}\left(\sum_{i=1}^{t} \rho_{i} u_{i}\right)=0
$$


e como $\sum_{i=1}^{t} \rho_{i} u_{i} \in V_{\alpha, k}$, segue da injetividade de $\left.\varphi\right|_{V_{\alpha, k}}$, que $\sum_{i=1}^{t} \rho_{i} u_{i}=0$. Logo concluímos que $\rho_{i}=0$, para $i=1, \cdots, t$. Assim

$$
\sum_{i=1}^{t} \beta_{i} u_{i}+\lambda_{i} b u_{i}=0 .
$$

Segue da primeira parte da demonstração que $\beta_{i}=\lambda_{i}=0$, para $i=1, \ldots, t$.

Portanto, dada uma base $\left\{u_{1}, u_{2}, \ldots, u_{t_{k}}\right\}$ para $V_{\alpha, k}$, temos que o conjunto $\left\{u_{1}, b u_{1}, b b u_{1}, u_{2}, b u_{2}, b b u_{2}, \ldots, u_{t_{k}}, b u_{t_{k}}, b b u_{t_{k}}\right\}$ é um conjunto linearmente independente que gera $W_{\alpha, k}$, o que mostra que é uma base para $W_{\alpha, k}$.

Teorema 2.2 Seja $\Phi=\left\{u_{1}, u_{2}, \ldots, u_{t_{\alpha}}\right\}$ uma base para $V_{\alpha}$ com $\alpha \neq$ 0 e $A=\left[\alpha_{i j}\right]$ a matriz da restrição de $\varphi$ em $V_{\alpha}$ em relação à base $\Phi$. Então a matriz da restrição de $L_{a}$ e $L_{b}$ em $W_{\alpha}$ em relação à base $\Phi^{\prime}=$ $\left\{u_{1}, b u_{1}, b b u_{1}, u_{2}, \ldots, b b u_{t_{\alpha}}\right\}$ é respectivamente

$$
A \otimes\left(\begin{array}{rrr}
0 & 1 & 0 \\
0 & 0 & -1 \\
0 & 0 & 0
\end{array}\right), \quad I_{t_{\alpha}} \otimes\left(\begin{array}{lll}
0 & 0 & 0 \\
1 & 0 & 0 \\
0 & 1 & 0
\end{array}\right),
$$

onde $I_{t_{\alpha}}$ é a matriz identidade de ordem $t_{\alpha} \times t_{\alpha}$.

Prova: Temos que $a u_{j}=0, a b u_{j}=\varphi\left(u_{j}\right)=\sum_{i=1}^{t_{\alpha}} \alpha_{i j} u_{i}$ e $a b b u_{j}=-b a b u_{j}-$ $b b a u_{j}=-b a b u_{j}=-b\left(\varphi\left(u_{j}\right)\right)=-b\left(\sum_{i=1}^{t_{\alpha}} \alpha_{i j} u_{i}\right)=\sum_{i=1}^{t_{\alpha}}-\alpha_{i j} b u_{i}$. Isto prova o teorema.

Tendo em vista que os $W_{\alpha}$ foram definidos para $\alpha \in F^{*}$, passaremos agora a definir $W_{0}$. Definiremos uma cadeia de $\mathcal{A}$-submódulos $W_{0, k}$, para $k \geq 1$, e finalmente a partir destes, definiremos $W_{0}$. Sejam

$$
\begin{aligned}
W_{0,0} & :=0, \\
W_{0,1} & :=\{x \in M \mid a x=b x=0\}, \\
& \vdots \\
W_{0, k+1} & :=\left\{x \in M \mid a x, b x \in W_{0, k}\right\} .
\end{aligned}
$$


Então definimos

$$
W_{0}:=\bigcup_{k=0}^{n} W_{0, k}
$$

onde $n$ é a dimensão de $M$. Segue imediatamente da definição que $W_{0}$ é invariante por $L_{a}$ e $L_{b}$, ou seja, $W_{0}$ é um $\mathcal{A}$-submódulo, e

$$
0=W_{0,0} \subset W_{0,1} \subset \cdots \subset W_{0, n}=W_{0} .
$$

Diferente do caso $W_{\alpha}$, para $\alpha \neq 0$, não é verdade que $W_{0}$ possa ser escrito como a soma $V_{0}+b V_{0}+b b V_{0}$, neste caso temos apenas a inclusão $V_{0}+b V_{0}+b b V_{0} \subseteq W_{0}$, pois podem existir elementos $x \in W_{0}$ tal que $x \notin$ $V_{0}+b V_{0}+b b V_{0}$. De fato no exemplo de Suttles, existe um elementos $x \in W_{0}$ tal que $x \notin V_{0}+b V_{0}+b b V_{0}$, o qual exibiremos abaixo. Lembremos que neste exemplo temos uma álgebra com base $\left\{e_{1}, e_{2}, e_{3}, e_{4}, e_{5}\right\}$ e os produtos não nulos são dados por

$$
e_{1} e_{2}=e_{2} e_{4}=-e_{1} e_{5}=e_{3}, \quad e_{1} e_{3}=e_{4} \quad \text { e } \quad e_{2} e_{3}=e_{5}
$$

Seja $\mathcal{A}=\left\langle e_{4}, e_{5}\right\rangle$ sendo $a=e_{4}$ e $b=e_{5}$. Então é claro que $V=$ $\left\langle e_{1}, e_{3}, e_{4}, e_{5}\right\rangle$ e dado que $e_{5} e_{4} e_{1}=e_{5} e_{4} e_{3}=e_{5} e_{4} e_{4}=e_{5} e_{4} e_{5}=0$, ou seja, $a b e_{i}=0$, para $i \neq 2$, temos que $V=V_{0}, b V_{0}=\left\{e_{3}\right\}$ e $b b V_{0}=\{0\}$, isto é, $V=V_{0}+b V_{0}+b b V_{0}$. Como $a a e_{2}=e_{4} e_{4} e_{2}=e_{4} e_{3}=0$ e $b a e_{2}=e_{5} e_{4} e_{2}=$ $e_{5} e_{3}=0$, segue que $a e_{2} \in W_{0,1}$ e uma vez que $b e_{2}=0 \in W_{0,1}$, temos que $e_{2} \in W_{0,2}$, isto é, $e_{2} \in W_{0}$ e $e_{2} \notin V_{0}+b V_{0}+b b V_{0}$.

\section{Lema 2.3}

$$
V_{0}+b V_{0}+b b V_{0} \subset W_{0}
$$

Prova: Basta mostrar que $V_{0} \subset W_{0}$, já que $W_{0}$ é um $\mathcal{A}$-submódulo. Para mostrar que $V_{0} \subset W_{0}$, mostraremos que $V_{0, k} \subset W_{0}$ por indução sobre $k \geq 0$. $\mathrm{O}$ caso $k=0$ é trivial. Seja $k \geq 0$ e suponhamos, por hipótese de indução, que

$$
V_{0, t} \subset W_{0} \text { para } 0 \leq t \leq k,
$$


verifiquemos que $V_{0, k+1} \subset W_{0}$. Seja $u \in V_{0, k+1}$. Então $\varphi^{k+1} u=0$ e portanto $\varphi^{k}(a b u)=0$, o que implica que $a b u \in V_{0, k}$ e temos então pela hipótese de indução que $a b u \in V_{0, k} \subset W_{0}$, logo $b a b u \in b V_{0, k} \subset W_{0}$. Mas $b a b u=-a b b u$, $\operatorname{logo} a b b u \in W_{0}$ e $b b b u=0$ do que segue que $b b u \in W_{0}$. De $b b u, a b u \in W_{0}$ segue que $b u \in W_{0}$ e como $a u=0$ temos que $u \in W_{0}$. Portanto, para todo $u \in V_{0, k+1}$, temos que $u \in W_{0}$, logo $V_{0, k+1} \subset W_{0}$. Assim, $V_{0} \subset W_{0}$ e dessa forma fica mostrado que

$$
V_{0}+b V_{0}+b b V_{0} \subset W_{0}
$$

\section{$2.2 \mathcal{A}$-módulos irredutíveis.}

A seguir caracterizaremos os $\mathcal{A}$-módulos irredutíveis em $\mathfrak{M}$. Observamos que a categoria dos $\mathcal{A}$-módulos na variedade $\mathfrak{M}$ é equivalente à categoria dos módulos associativos sobre a álgebra $A s s[x, y] / I$, onde $A s s[x, y]$ é a álgebra livre associativa gerada por $x$ e $y$ e $I$ é o ideal gerado pelo conjunto $\left\{x^{3}, y^{3}, x y^{2}+x y x+y x^{2}, y x^{2}+y x y+x y^{2}\right\}$.

Notamos que se $0 \neq v \in V_{\alpha}$ e $\varphi(v)=\alpha v$, então $I=\langle v, b v, b b v\rangle$ é um submódulo de $M$, pois é claro que $I$ é invariante por $L_{b}$ e que $a b v=\alpha v \in I$ e $a b b v=-b a b v-b b a v=-\alpha b v \in I$, sendo também invariante por $L_{a}$. Como uma consequência do que fizemos anteriormente temos que se $M$ é um $\mathcal{A}$-módulo irredutível, então $\varphi$ tem apenas um autovalor $\alpha$ e $M=W_{\alpha} \subset$ $\langle v, b v, b b v\rangle$, para algum $v \in V_{\alpha}$. Portanto, um $\mathcal{A}$-módulo irredutível tem dimensão menor ou igual que 3 .

Lema 2.4 Se $M$ é um $\mathcal{A}$-módulo irredutível, então $\operatorname{dim}(M)=1$ ou $\operatorname{dim}(M)$ $=3$.

Prova: Sabemos que para autovetor $v$ de $\varphi$ o subespaço gerado por $v, b v$ e $b b v$ é um $\mathcal{A}$-submódulo de $M$. Assim, sendo que $M$ é irredutível, temos que $\varphi$ 
tem exatamente um autovalor que denotaremos por $\alpha$. Seja $v$ um autovetor de $\varphi$ associado ao autovalor $\alpha$. Pelas observações acima $\langle v, b v, b b v\rangle$ e um $\mathcal{A}$-submódulo de $M$. Já que $M$ é irredutível $M=\langle v, b v, b b v\rangle$. Analisaremos os casos $\alpha=0$ e $\alpha \neq 0$.

Se $\alpha \neq 0$, então pelo Teorema 2.1 o conjunto $\{v, b v, b b v\}$ é uma base de $M$ e $a v=0, a(b v)=\alpha v$ e $a(b b v)=-b a b v=-\alpha b v$.

Assumiremos agora que $\alpha=0$. Se $b b v$ for diferente de zero, então a família $\{v, b v, b b v\}$ é linearmente independente e dado que $a b b v=-b a b v-b b a v=$ $-b(\varphi(v))=0$ e $b(b b v)=0$ temos que $\langle b b v\rangle$ é um $\mathcal{A}$-submódulo próprio de $M$ em contradição com a hipótese. Portanto $b b v=0$. Analogamente, se $b v$ for diferente de zero, então temos que $a(b v)=\varphi(v)=0$ e $b(b v)=b b v=0$ $\operatorname{logo}\langle b v\rangle$ é um $\mathcal{A}$-submódulo próprio de $M$ em contradição com a hipótese. Assim $b v=0$. Consequentemente, $M=\langle v\rangle$, tem dimensão 1.

Corolário 2.5 Se $M$ é um $\mathcal{A}$-módulo irredutivel de dimensão 3, então para uma base adequada, as ações de a e de b são dadas por

$$
L_{a} \longrightarrow\left(\begin{array}{ccc}
0 & \alpha & 0 \\
0 & 0 & -\alpha \\
0 & 0 & 0
\end{array}\right) \quad e \quad L_{b} \longrightarrow\left(\begin{array}{ccc}
0 & 0 & 0 \\
1 & 0 & 0 \\
0 & 1 & 0
\end{array}\right)
$$

$\operatorname{com} \alpha \neq 0$.

Para cada $\alpha \in F, \alpha \neq 0$, denotaremos por $I_{\alpha}$ o $\mathcal{A}$-módulo $\langle v, b v, b b v\rangle$, onde $a v=0, a b v=\alpha v$ e $a b b v=-\alpha b v$, isto é, as matrizes de $L_{a}$ e $L_{b}$ são dadas como acima. Por $I_{0}$ denotamos um $\mathcal{A}$-módulo irredutível de dimensão 1. Observamos que para cada $\alpha \in \Delta$, existe uma cadeia de $\mathcal{A}$-submódulos de $W_{\alpha}$

$$
0=U_{\alpha, 0} \subset U_{\alpha, 1} \subset \cdots \subset U_{\alpha, n_{k}}=W_{\alpha},
$$

tal que

$$
\frac{U_{\alpha, i}}{U_{\alpha, i-1}} \cong \bigoplus I_{\alpha}
$$

para $i=1,2, \ldots, n_{k}$. Mostraremos na seguinte subseção que $W_{\alpha}$ é o submódulo maximal de $M$ que satisfaz a propriedade acima. 


\subsection{Decomposição de $M$ como soma $\operatorname{dos} W_{\alpha}$}

Queremos agora mostrar, que todo $\mathcal{A}$-módulo na classe $\mathfrak{M}$, de dimensão finita, pode ser decomposto como soma direta dos $W_{\alpha}$. Mostraremos primeiro que a soma dos $W_{\alpha}$, para todo $\alpha \in F$, é direta e logo enunciaremos dois lemas que precisaremos para a demonstração do teorema.

Nesta seção, $M$ será um $\mathcal{A}$-módulo na classe $\mathfrak{M}$ de dimensão finita $n$, e para cada inteiro não negativo $k$ e cada $\alpha$ no corpo algebricamente fechado $F$ os espaços vetoriais $V_{\alpha, k}, V_{\alpha}$ e os $\mathcal{A}$-submódulos $W_{\alpha, k}$ e $W_{\alpha}$ definidos neste capítulo. Denotaremos por $\Delta$ o conjunto finito formado por todos os autovalores de $\varphi$ e por $\Delta^{*}$ o conjunto de todos os autovalores de $\varphi$ diferentes de zero.

Lema 2.6 Temos que

$$
\sum_{\alpha \in \Delta^{*}} W_{\alpha}=\bigoplus_{\alpha \in \Delta^{*}} W_{\alpha}
$$

Prova: Seja $\Delta^{*}=\left\{\alpha_{1}, \ldots, \alpha_{k}\right\}$. Como $\sum_{i=1}^{k} W_{\alpha_{i}}=\sum_{i=1}^{k} V_{\alpha_{i}}+b V_{\alpha_{i}}+b b V_{\alpha_{i}}$, para mostrar que a soma é direta basta mostrar que se $\sum_{i=1}^{k} u_{i}+b v_{i}+b b w_{i}=$ 0 , para $u_{i}, v_{i}, w_{i} \in V_{\alpha_{i}}$, então $u_{i}=v_{i}=w_{i}=0$, para $i=1, \ldots, k$.

Vejamos primeiro que se $\sum_{i=1}^{k} u_{i}+b v_{i}=0$, para $u_{i}, v_{i} \in V_{\alpha_{i}}$, então $u_{i}=v_{i}=0$, para $i=1, \ldots, k$. Seja $\sum_{i=1}^{k} u_{i}+b v_{i}=0$. Então $0=$ $\sum_{i=1}^{k} a u_{i}+a b v_{i}=\sum_{i=1}^{k} a b v_{i}$, pois $a u_{i}=0$, para $i=1, \ldots, k . \quad \operatorname{Logo} 0=$ $\sum_{i=1}^{k} a b v_{i} \in \bigoplus_{i=1}^{k} V_{\alpha_{i}}$, donde segue que $\varphi\left(v_{i}\right)=a b v_{i}=0$, para $i=1, \ldots, k$, mas $\varphi$ é injetora em $V_{\alpha_{i}}$, pois $\alpha_{i} \neq 0, \operatorname{logo} v_{i}=0$, para $i=1, \ldots, k$. Assim $0=\sum_{i=1}^{k} u_{i}+b v_{i}=\sum_{i=1}^{k} u_{i} \in \bigoplus_{i=1}^{k} V_{\alpha_{i}}$ e portanto $u_{i}=0$, para $i=1, \ldots, k$.

Agora, seja $\sum_{i=1}^{k} u_{i}+b v_{i}+b b w_{i}=0$, para $u_{i}, v_{i}, w_{i} \in V_{\alpha_{i}} . \quad$ Então $0=\sum_{i=1}^{k} a u_{i}+a b v_{i}+a b b w_{i}=\sum_{i=1}^{k} a b v_{i}-b a b w_{i}$ e uma vez que $a b v_{i}$ e $a b w_{i}$ estão em $V_{\alpha_{i}}$, temos da primeira parte da demonstração que $a b v_{i}=a b w_{i}=0$, para $i=1, \ldots, k, \operatorname{logo} v_{i}=w_{i}=0$ pela injetividade de $\varphi$ em $V_{\alpha_{i}}$, assim $0=\sum_{i=1}^{k} u_{i} \in \bigoplus_{i=1}^{k} V_{\alpha_{i}}$ e portanto $u_{i}=0$, para $i=1, \ldots, k$. 
Lema 2.7 Temos que

$$
\sum_{\alpha \in \Delta} W_{\alpha}=\bigoplus_{\alpha \in \Delta} W_{\alpha}
$$

Prova: Pelo lema anterior, basta mostrar que

$$
W_{0} \cap\left(\sum_{i=1}^{k} W_{\alpha_{i}}\right)=0,
$$

onde $\Delta^{*}=\left\{\alpha_{1}, \ldots, \alpha_{k}\right\}$. Provaremos por indução sobre $j$ que

$$
W_{0, j} \cap\left(\sum_{i=1}^{k} W_{\alpha_{i}}\right)=0 .
$$

O caso $j=0$ é trivial. Seja agora $j>0$ e assumir por hipótese de indução que $W_{0, s} \cap\left(\sum_{i=1}^{k} W_{\alpha_{i}}\right)=0$ para todo inteiro não negativo $s<j$. Seja $x \in W_{0, j} \cap\left(\sum_{i=1}^{k} W_{\alpha_{i}}\right)=W_{0, j} \cap\left(\sum_{i=1}^{k} V_{\alpha_{i}}+b V_{\alpha_{i}}+b b V_{\alpha_{i}}\right)$. Então podemos exprimir $x$ sob a forma

$$
x=\sum_{i=1}^{k} u_{i}+b v_{i}+b b w_{i},
$$

com $u_{i}, v_{i}, w_{i} \in V_{\alpha_{i}}$. Multiplicando por $b$ duas vezes e seguidamente duas vezes por $a$, obtemos $a a b b x=\sum_{i=1}^{k} a a b b u_{i} \in W_{0, j-1} \cap\left(\sum_{i=1}^{k} W_{\alpha_{i}}\right)=0$, logo $0=\operatorname{aabb}\left(\sum_{i=1}^{k} u_{i}\right)=-\varphi^{2}\left(\sum_{i=1}^{k} u_{i}\right)$ e portanto $\sum_{i=1}^{k} u_{i}=0$ já que a restrição de $\varphi$ a $\sum_{i=1}^{k} W_{\alpha_{i}}$ é injetora. Isto implica que $u_{1}=u_{2}=\cdots=u_{k}=0$.

De maneira análoga podemos multiplicar pela esquerda ambos membros da igualdade (2.2) primeiro por $b$ e depois duas vezes por $a$ e obtemos que $a a b x=\sum_{i=1}^{k} a a b b v_{i} \in W_{0, j-1} \cap\left(\sum_{i=1}^{k} W_{\alpha_{i}}\right)=0, \operatorname{logo} 0=a a b b\left(\sum_{i=1}^{k} v_{i}\right)=$ $-\varphi^{2}\left(\sum_{i=1}^{k} v_{i}\right)$ e portanto $\sum_{i=1}^{k} v_{i}=0$ já que a restrição de $\varphi$ a $\sum_{i=1}^{k} W_{\alpha_{i}}$ é injetora. Isto implica que $v_{1}=v_{2}=\cdots=v_{k}=0$. Finalmente, multiplicando pela esquerda ambos membros da identidade (2.2) por $a$ duas vezes, podemos provar de maneira análoga que $w_{1}=w_{2}=\cdots=w_{k}=0$. Isto prova o lema. 
Lema 2.8 Seja I um $\mathcal{A}$-submódulo irredutível de $M$, tal que $I \cong I_{\alpha}$. Então

i) Se au $\in I$ para $u \in M$, então existe $s \in I$ tal que $a(u+s)=0$.

ii) Se au,bu $\in I$ para $u \in M$, então existe $s_{1} \in I$ tal que $a\left(u+s_{1}\right)=0$ e $b\left(u+s_{1}\right)=0$.

\section{Prova:}

i) Seja $a u=\lambda_{1} v+\lambda_{2} b v+\lambda_{3} b b v$ para $\lambda_{1}, \lambda_{2}, \lambda_{3} \in F$. Então $0=a a a u=$ $-\lambda_{3} \alpha^{2} v, \log \mathrm{o} \lambda_{3}=0$. Portanto se $s=-\frac{\lambda_{1}}{\alpha} b v+\frac{\lambda_{2}}{\alpha} b b v$, então $a(u+s)=0$.

ii) Seja $b u \in I$. Então se $u_{1}=u+s$ com $s \in I$, temos que $b u_{1} \in$ I. Sejam $\beta_{1}, \beta_{2}, \beta_{3} \in F$ tal que $b u_{1}=\beta_{1} v+\beta_{2} b v+\beta_{3} b b v$. Então $0=a a a b u_{1}=-\beta_{3} \alpha^{2} v, \operatorname{logo} \beta_{3}=0$. Agora $\alpha \beta_{1} v-\alpha \beta_{2} b v=\beta_{1} a b v+$ $\beta_{2} a b b v=a b\left(\beta_{1} v+\beta_{2} b v\right)=a b b u_{1}=-b a b u_{1}=-b\left(\beta_{2} \alpha v\right)=-\alpha \beta_{2} b v$, $\operatorname{logo} \beta_{1}=0$ e assim $b u_{1}=\beta_{2} b v$. Portanto $a u_{2}=b u_{2}=0$ para $u_{2}=u_{1}-\beta_{2} v$.

\section{Teorema 2.9}

$$
M=\bigoplus_{\alpha \in \Delta} W_{\alpha}
$$

Prova: Demonstraremos este teorema por indução sobre $n$, sendo $n$ a dimensão do $\mathcal{A}$-módulo $M$. A demonstração é trivial para os casos $n=0,1$. Seja $M$ um $\mathcal{A}$-módulo de potências associativas de dimensão $n>1$ e suponhamos o teorema para todo $\mathcal{A}$-módulo de potências associativas de dimensão menor a $n$. Dividiremos a prova em dois casos.

- Caso 1: Suponhamos que $0 \in \Delta$. Então existe $v \in W_{0,1}$ com $v \neq 0$. Seja $J$ o $\mathcal{A}$-submódulo $\langle v\rangle$ e $\bar{M}$ o $\mathcal{A}$-submódulo quociente de p.a. $M / J$. É fácil ver que $\bar{W}_{0}=W_{0} / J$. Mostraremos agora que para cada $\alpha \neq 0$ temos que $\bar{W}_{\alpha}=\left(W_{\alpha}+J\right) / J$, para isto basta mostrar que $\bar{V}_{\alpha}=\left(V_{\alpha}+J\right) / J$. Demonstraremos por indução sobre $k$ que $\overline{V_{\alpha, k}}=$ 
$\left(V_{\alpha, k}+J\right) / J$. Para $k=0$ a demonstração é trivial. Seja $k>0$ e suponhamos por hipótese de indução que $\bar{V}_{\alpha, k-1}=\left(V_{\alpha, k-1}+J\right) / J$. Se $u+J \in \bar{V}_{\alpha, k}$, então $\varphi(u+J)-\alpha(u+J)=(a b u-\alpha u)+J \in$ $\bar{V}_{\alpha, k-1}=\left(V_{\alpha, k-1}+J\right) / J$. Portanto $a b u=\alpha u+u_{k-1}+\lambda v$ para algum $u_{k-1} \in V_{\alpha, k-1}$ e $\lambda \in F$. Logo $u+\frac{\lambda}{\alpha} v \in V_{\alpha, k}$, do que segue que $u+J \in$ $\left(V_{\alpha, k}+J\right) / J$. Contrariamente, se $u \in V_{\alpha, k}$, então $a b(u+J)-\alpha(u+J)=$ $(a b u-\alpha u)+J \in\left(V_{\alpha, k-1}+J\right) / J=\bar{V}_{\alpha, k-1}$ e portanto $u+J \in \bar{V}_{\alpha, k}$. Por hipótese de indução temos que $\bar{M}=\sum_{\alpha \in \Delta} \bar{W}_{\alpha}=\left(W_{\alpha}+J\right) / J$. Assim $M=\sum_{\alpha \in \Delta} W_{\alpha}$, uma vez que $J \subset W_{0}$.

- Caso 2: Suponhamos que $0 \notin \Delta$. Seja $J=\langle v, b v, b b v\rangle$ um $\mathcal{A}$-submódulo irredutível de $M$ tal que $J \cong J_{\alpha}$. Denotamos por $\bar{M}$ o $\mathcal{A}$-submódulo quociente $M / J$. Pelos lemas 2.3 e 2.8 , temos que $\bar{W}_{0}=\{0\}+J=$ $\left(W_{0}+J\right) / J$ e pode-se mostrar facilmente que $\bar{W}_{\alpha}=\left(W_{\alpha}+J\right) / J$. Seja $\beta \in F \operatorname{com} \beta \neq 0, \alpha$. Provaremos por indução sobre $k$ que $\bar{V}_{\beta, k}=\left(V_{\beta, k}+J\right) / J$ para todo inteiro não negativo $k$. O caso $k=0$ é trivial. Seja $k>0$ e suponhamos por hipótese de indução que $\bar{V}_{\beta, k-1}=\left(V_{\beta, k-1}+J\right) / J$. Seja $u+J \in \bar{V}_{\beta, k}$. Então au $\in J$ e pelo Lema 2.8, existe $u_{1} \in M$ tal que $u-u_{1} \in J$ e $a u_{1}=0$. Pela hipótese de indução temos que $\varphi(u)-\beta u \in\left(V_{\beta, k-1}+J\right) / J$, logo $\varphi\left(u_{1}\right)=\beta u_{1}+u^{\prime}+\lambda_{1} v+\lambda_{2} b v+\lambda_{3} b b v$ para algum $u^{\prime} \in V_{\beta, k-1}$ e $\lambda_{1}, \lambda_{2}, \lambda_{3} \in F$. Portanto, $0=a \varphi\left(u_{1}\right)=\lambda_{2} \alpha v-\lambda_{3} \alpha b v$ e segue que $\lambda_{2}=\lambda_{3}=0$. Uma vez que é possível verificar que $u_{1}+\frac{\mu}{\alpha-\beta} v \in V_{\beta, k}$ temos que $u+J \in\left(V_{\beta, k}+J\right) / J$. Assim $\bar{V}_{\beta, k} \subset\left(V_{\beta, k}+J\right) / J$, o que implica que $\bar{W}_{\beta} \subset\left(W_{\beta}+J\right) / J$ para todo $\beta \in F$. A prova da inclusão contraria é imediata, portanto temos que $\bar{W}_{\beta}=\left(W_{\beta}+J\right) / J$. Logo pela hipótese de indução temos que $\bar{M}=\sum_{\gamma \in F} \bar{W}_{\gamma}=\sum_{\gamma \in F}\left(W_{\gamma}+J\right) / J$. Já que $J \subset W_{\alpha}$, segue que $M=\sum_{\gamma \in F} W_{\gamma}$.

Isto demonstra o teorema.

Observamos que a decomposição do $\mathcal{A}$-módulo $M$, independe da escolha 
da base de $\mathcal{A}$. Isto é, se escolhemos a base $\left\{a^{\prime}, b^{\prime}\right\}$ de $\mathcal{A}$, então

$$
\left(\begin{array}{l}
a^{\prime} \\
b^{\prime}
\end{array}\right)=P^{t}\left(\begin{array}{l}
a \\
b
\end{array}\right)
$$

com $P \in M_{2 \times 2}(F)$ invertível. Assim $V^{\prime}=\operatorname{ker} L_{a^{\prime}}, \quad \varphi^{\prime}=\left.L_{a^{\prime}} L_{b^{\prime}}\right|_{V^{\prime}}$ e os autovalores de $\varphi^{\prime}$ são dados pelo conjunto $\Delta^{\prime}=\left\{\alpha \alpha_{1}, \ldots, \alpha \alpha_{k}\right\}$, sendo que $\alpha=\operatorname{det}(P)$ e $\Delta=\left\{\alpha_{1}, \ldots, \alpha_{k}\right\}$ é o conjunto de autovalores de $\varphi$. 
Capítulo 3

\section{Nilálgebras de potências associativas e nilíndice 4}

No capítulo anterior vimos que, se $F$ é um corpo algebricamente fechado de característica diferente de 2 e $3, \mathfrak{M}$ a variedade das álgebras comutativas de potências associativas sobre $F$ e $\mathcal{A}$ é uma álgebra trivial de dimensão 2 , então todo $\mathcal{A}$-módulo $M$ de dimensão finita na variedade $\mathfrak{M}$ pode ser decomposto como soma direta dos $\mathcal{A}$-submódulos $W_{\alpha}$, com $\alpha \in F$. Neste capítulo utilizaremos este resultado para estudar a estrutura das nilálgebras comutativas de potências associativas de dimensão finita. Para tal seja $\mathfrak{M}$ a variedade das nilálgebras comutativas de potências associativas e nilíndice quatro sobre o corpo $F$, isto é a variedade das nilálgebras comutativas de potências associativas verificando a identidade

$$
x^{4}=x^{2} x^{2}=0
$$

Seja $\mathcal{B} \in \mathfrak{M}$ e $n=\operatorname{dim} \mathcal{B}$. Se o nilíndice de $\mathcal{B} \leq 3$, então $\mathcal{B}$ é nilpotente. Se o nilíndice de $\mathcal{B}=4$, então $\mathcal{B}$ possui uma subálgebra trivial de dimensão 2. Seja $\mathcal{A}$ uma subálgebra trivial de $\mathcal{B}$ de dimensão 2. É fácil ver que $\mathcal{B}$ é um $\mathcal{A}$-módulo em $\mathfrak{M}$ via o produto usual em $\mathcal{B}$, since a álgebra $\mathcal{C}=\mathcal{A} \oplus \mathcal{B}$ com produto definido por $\left(x, b_{1}\right)\left(y, b_{2}\right)=\left(x y, x b_{2}+y b_{1}\right)=\left(0, x b_{2}+y b_{1}\right)$, para todo $x, y \in \mathcal{A}$ e $b_{1}, b_{2} \in \mathcal{B}$, está na variedade $\mathfrak{M}$. Uma vez escolhida $\mathcal{A} \subset \mathcal{B}$ e fixados os elementos $a, b \in \mathcal{A}$, obtemos uma decomposição de $\mathcal{B}$ como soma 
direta dos $\mathcal{A}$-submódulos $W_{\alpha}$, isto é

$$
\mathcal{B}=\bigoplus_{\alpha \in F} W_{\alpha}
$$

Em vista disto, estamos interessados em conhecer os produtos $W_{\alpha} \cdot W_{\beta}$ em $\mathcal{B}$ para todos os $\alpha, \beta \in F$. Ao longo deste capítulo os elementos $a, b$ continuarão fixos, isto é $a, b \in \mathcal{A} \subset \mathcal{B}$ e $a^{2}=b^{2}=a b=0$.

Para todo $x \in \mathcal{B}$, temos que $x^{2} x^{2}=0$, que linearizando nos leva a

$$
x^{2}(x y)=0 .
$$

Linearizando (3.1) em $x$ temos

$$
(x y)(x z)=-\frac{1}{2} x^{2}(y z)
$$

e

$$
(x y)(z w)+(x z)(y w)+(x w)(y z)=0 .
$$

Linearizando a identidade $x^{4}=0$, obtemos as seguintes três novas identidades,

$$
\begin{aligned}
& 2 x(y(x y))+2 y(x(x y))+x\left(x y^{2}\right)+y\left(y x^{2}\right)=0, \\
& 2 x(x(y z))+2 x(y(x z))+ 2 x(z(x y))+2 y(x(x z))+ \\
& 2 z(x(x y))+y\left(z x^{2}\right)+z\left(y x^{2}\right)=0,
\end{aligned}
$$

e

$$
\begin{array}{r}
x(y(z w))+x(z(y w))+x(w(y z))+y(x(z w))+y(z(x w))+ \\
y(w(x z))+z(x(y w))+z(y(x w))+z(w(x y))+ \\
w(x(y z))+w(y(x z))+w(z(x y))=0 .
\end{array}
$$

Notemos que as identidades anteriores verificam-se para todo $x, y, z, w \in$ B. Para facilitar a notação, na ausência de parênteses entenderemos o produto da direita para a esquerda, isto é escreveremos $x y z w$ ao invés de $x(y(z w))$ e no caso $(x y)(z w)$ escreveremos $x w \cdot y z$; caso seja necessário, os parênteses serão indicados. 


\section{$3.1 \quad \mathrm{O}$ produto $W_{\alpha} \cdot W_{\beta}$ para $\alpha, \beta \in F^{*}$}

Passaremos agora ao calculo dos produtos $W_{\alpha} \cdot W_{\beta}$, onde pode ocorrer que $\alpha \beta \neq 0, \operatorname{com} \alpha \neq \beta, \alpha=\beta \neq 0, \beta=0$ e $\alpha \neq 0$ e finalmente $\alpha=\beta=0$. Assim temos que analisar estes quatro casos.

Inicialmente estudaremos o produto $W_{\alpha} \cdot W_{\beta}$, para $\alpha, \beta \neq 0$. Observamos que para todo $\alpha \in F^{*}$, e para todo inteiro não negativo, temos que

$$
W_{\alpha}=V_{\alpha}+b V_{\alpha}+b b V_{\alpha}
$$

e

$$
W_{\alpha, k}=V_{\alpha, k}+b V_{\alpha, k}+b b V_{\alpha, k} .
$$

O Teorema 2.1, nos diz que basta calcular os produtos uv, ubv, ubbv, bu . $b v, b u \cdot b b v$ e $b b u \cdot b b v$, onde $u \in U_{\alpha, k}$ e $v \in U_{\beta, m}$, sendo que o conjunto $U_{\alpha, k}=\left\{u_{11}, u_{12}, \ldots, u_{1 t_{1}}, \ldots, u_{k 1}, u_{k 2}, \ldots, u_{k t_{k}}\right\}$ é uma base para $V_{\alpha, k}$. Em algumas ocasiões será necessário tomar um elemento mais específico do conjunto $U_{\alpha, k}$. Portanto é importante ressaltar, que cada vez que seja mostrada alguma identidade e/ou propriedade para um elemento $u_{i} \in V_{\alpha, i}$, estamos considerando $u_{i}$ como um elemento do conjunto $\left\{u_{i 1}, u_{i 2}, \ldots, u_{i t_{i}}\right\}$, satisfazendo

$$
a u_{i}=0 \quad \text { e } \quad a b u_{i}=\alpha u_{i}+x_{i-1},
$$

para $i \geq 1$, sendo que $x_{i-1}$ é uma combinação linear de elementos de $U_{\alpha, i-1}$. Lembrando que $a b b u_{i}=-b a b u_{i}$, temos também a igualdade

$$
a b b u_{i}=-\alpha b u_{i}-b x_{i-1} .
$$

Das igualdades anteriores é claro que $V_{\alpha}$ e $b V_{\alpha}$ são invariantes por $\varphi$, isto é $a b u \in V_{\alpha}$ e $a b b u \in b V_{\alpha}$ para todo $u \in V_{\alpha}$.

Notemos que pela identidade (3.2) temos

$$
\begin{aligned}
b b u \cdot b b v & =-\frac{1}{2} b^{2}(b u \cdot b v)=0, \\
b b u \cdot b v & =-\frac{1}{2} b^{2} \cdot v b u=0, \\
b u \cdot b v & =-\frac{1}{2} b^{2} \cdot u v=0 .
\end{aligned}
$$


Logo

$$
b b u \cdot b b v=b b u \cdot b v=b u \cdot b v=0,
$$

para todo $u \in V_{\alpha}$ e $v \in V_{\beta}$.

Isto prova o seguinte:

Lema 3.1 Se $\alpha, \beta \in F \operatorname{com} \alpha \beta \neq 0$, então

$$
b V_{\alpha} \cdot b V_{\beta}=\{0\}, \quad b V_{\alpha} \cdot b b V_{\beta}=\{0\} \quad \text { e } \quad b b V_{\alpha} \cdot b b V_{\beta}=\{0\} .
$$

Continuaremos agora com o cálculo dos produtos $V_{\alpha} \cdot V_{\beta}$ e $V_{\alpha} \cdot b V_{\beta}$.

Lema 3.2 Se $\alpha, \beta \in F \operatorname{com} \alpha \beta \neq 0$, então

$$
V_{\alpha} \cdot V_{\beta}=\{0\} \quad \text { e } \quad V_{\alpha} \cdot b V_{\beta}=\{0\} .
$$

Prova: Como $V_{\alpha, n}=V_{\alpha}$, basta mostrar que

$$
V_{\alpha, i} \cdot V_{\beta, j}=\{0\} \quad \text { e } \quad V_{\alpha, i} \cdot b V_{\beta, j}=\{0\}, \quad \text { para } \quad i, j \geq 1 .
$$

Demonstraremos as duas igualdades anteriores por indução sobre $k=i+j$.

Seja $k=2$. Se $i=0$ ou $j=0$ então a igualdade é trivial já que por definição $V_{\alpha, 0}=0$. Consideremos agora o caso $i=j=1$. Se $u_{1} \in V_{\alpha, 1}$ e $v_{1} \in$ $V_{\beta, 1}$, então por (3.7) temos que $a u_{1}=0, a b u_{1}=\alpha u_{1}, a v_{1}=0$ e $a b v_{1}=\beta v_{1}$, logo por (3.2), (3.3) e lembrando que $a b=0$, temos que

$$
u_{1} v_{1}=\frac{1}{\alpha \beta}\left(a b u_{1}\right)\left(a b v_{1}\right)=-\frac{1}{2 \alpha \beta} a^{2}\left(b u_{1} \cdot b v_{1}\right)=0
$$

e

$$
u_{1} \cdot b v_{1}=\frac{1}{\alpha} a b u_{1} \cdot b v_{1}=-\frac{1}{\alpha}\left(a b \cdot v_{1} b u_{1}+a v_{1} \cdot b b u_{1}\right)=0 .
$$

Assim

$$
u_{1} v_{1}=u_{1} b v_{1}=0
$$


e portanto

$$
V_{\alpha, 1} \cdot V_{\beta, 1}=\{0\} \quad \text { e } \quad V_{\alpha, 1} \cdot b V_{\beta, 1}=\{0\} .
$$

Seja $k>2$ e suponhamos, por hipótese de indução, que

$$
V_{\alpha, t} \cdot V_{\beta, l}=\{0\} \quad \text { e } \quad V_{\alpha, t} \cdot b V_{\beta, l}=\{0\}, \quad \text { para } \quad 2 \leq t+l<k .
$$

Sejam $i$, e $j$ inteiros positivos tais que $i+j=k$ e considerar $u_{i} \in V_{\alpha, i}$ e $v_{j} \in V_{\beta, j}$. Então existem $x_{i-1} \in V_{\alpha, i-1}$ e $y_{j-1} \in V_{\beta, j-1}$ tais que

$$
a u_{i}=0, \quad a b u_{i}=\alpha u_{i}+x_{i-1}, \quad a v_{j}=0 \quad \text { e } \quad a b v_{j}=\beta v_{j}+y_{j-1} .
$$

Logo

$$
\begin{aligned}
u_{i} v_{j} & =\frac{1}{\alpha \beta}\left(a b u_{i}-x_{i-1}\right)\left(a b v_{j}-y_{j-1}\right) \\
& =\frac{1}{\alpha \beta}\left(a b u_{i} \cdot a b v_{j}-y_{j-1} a b u_{i}-x_{i-1} a b v_{j}+x_{i-1} y_{j-1}\right) .
\end{aligned}
$$

De (3.2) segue que $a b u_{i} \cdot a b v_{j}=0$, portanto

$$
u_{i} v_{j}=\frac{1}{\alpha \beta}\left[-y_{j-1}\left(\alpha u_{i}+x_{i-1}\right)-x_{i-1}\left(\beta v_{j}+y_{j-1}\right)+x_{i-1} y_{j-1}\right] .
$$

Então, pela hipótese de indução temos

$$
u_{i} v_{j}=-\frac{1}{\alpha \beta}\left(\alpha y_{j-1} u_{i}+\beta x_{i-1} v_{j}+x_{i-1} y_{j-1}\right)=0 .
$$

Temos também que

$$
\begin{aligned}
u_{i} b v_{j} & =\frac{1}{\alpha \beta}\left(a b u_{i}-x_{i-1}\right)\left(b a b v_{j}-b y_{j-1}\right) \\
& =-\frac{1}{\alpha \beta}\left(a b u_{i}-x_{i-1}\right)\left(a b b v_{j}+b y_{j-1}\right) \\
& =-\frac{1}{\alpha \beta}\left(a b u_{i} \cdot a b b v_{j}+a b u_{i} \cdot b y_{j-1}-x_{i-1} \cdot a b b v_{j}-x_{i-1} y_{j-1}\right) .
\end{aligned}
$$

Logo por (3.2) $a b u_{i} \cdot a b b v_{j}=0$ e pela hipótese de indução $a b u_{i} \cdot b y_{j-1}=$ $x_{i-1} \cdot b a b v_{j}=x_{i-1} y_{j-1}=0$, desta forma $u_{i} b v_{j}=0$ e temos então que

$$
V_{\alpha, i} \cdot V_{\beta, j}=\{0\} \quad \text { e } \quad V_{\alpha, i} \cdot b V_{\beta, j}=\{0\}, \quad \text { para } \quad i, j \geq 1,
$$


o que demonstra o lema.

Assim, para determinar completamente o produto $W_{\alpha} \cdot W_{\beta}$, resta apenas conhecer o produto $V_{\alpha} \cdot b b V_{\beta}$. Até aqui o produto foi calculado com a única restrição de $\alpha \beta \neq 0$, sem importar se $\alpha$ era igual ou diferente de $\beta$, mas neste produto que vamos calcular, faremos os dois casos separadamente, dado que cada um deles origina um resultado diferente, como veremos na continuação.

Lema 3.3 Se $\alpha, \beta \in F^{*}$ com $\alpha \neq \beta$, então

$$
V_{\alpha} \cdot b b V_{\beta}=\{0\} .
$$

Prova: Mostraremos, por indução sobre $k=i+j$, que

$$
V_{\alpha, i} \cdot b b V_{\beta, j}=\{0\}, \quad \text { para } i, j \geq 1
$$

Seja $k=2$, e portanto $i=j=1$. Se $u_{1} \in V_{\alpha, 1}$ e $v_{1} \in V_{\beta, 1}$, então por (3.3)

$$
\begin{gathered}
u_{1} b b v_{1}=\frac{1}{\alpha} a b u_{1} \cdot b b v_{1}=-\frac{1}{\alpha}\left[(a b)\left(b u_{1} \cdot b v_{1}\right)+a b v_{1} \cdot b b u_{1}\right] \\
=-\frac{1}{\alpha} a b v_{1} \cdot b b u_{1}=-\frac{\beta}{\alpha} v_{1} b b u_{1} .
\end{gathered}
$$

Por outro lado, temos da identidade (3.5) e os lemas 3.1 e 3.2 que

$$
\begin{aligned}
v_{1} b b u_{1} & =-\frac{1}{2}\left[2 b u_{1} b v_{1}+2 b b u_{1} v_{1}+2 b v_{1} b u_{1}+2 u_{1} b b v_{1}+u_{1} v_{1} b^{2}+v_{1} u_{1} b^{2}\right] \\
& =-u_{1} b b v_{1} .
\end{aligned}
$$

Logo $\alpha=\beta$ ou $u_{1} b b v_{1}=0$, mas por hipótese $\alpha \neq \beta$, o que nos leva a concluir que

$$
u_{1} b b v_{1}=0
$$

e portanto

$$
V_{\alpha, 1} \cdot b b V_{\beta, 1}=\{0\} .
$$

Seja $k>2$ e suponhamos, por hipótese de indução, que

$$
V_{\alpha, t} \cdot b b V_{\beta, l}=\{0\},
$$


para todos os inteiros não negativos $t$ e $l$ tais que $t+l<k$. Sejam $i$ e $j$ inteiros positivos tais que $i+j=k$ e considerar $u_{i} \in V_{\alpha, i}$ e $v_{j} \in V_{\beta, j}$. Então por (3.7) existem $x_{i-1} \in V_{\alpha, i-1}$ e $y_{j-1} \in V_{\beta, j-1}$ tais que

$$
a u_{i}=0, \quad a b u_{i}=\alpha u_{i}+x_{i-1}, a v_{j}=0 \quad \text { e } \quad a b v_{j}=\beta v_{j}+y_{j-1},
$$

e utilizando (3.3) temos que

$$
\begin{aligned}
u_{i} b b v_{j} & =\frac{1}{\alpha}\left[\left(a b u_{i}-x_{i-1}\right) b b v_{j}\right]=\frac{1}{\alpha}\left[a b u_{i} \cdot b b v_{j}-x_{i-1} b b v_{j}\right] \\
& =-\frac{1}{\alpha}\left[(a b)\left(b u_{i} \cdot b v_{j}\right)+a b v_{j} \cdot b b u_{i}+x_{i-1} b b v_{j}\right] \\
& =-\frac{1}{\alpha}\left[\left(\beta v_{j}+y_{j-1}\right) b b u_{i}+x_{i-1} b b v_{j}\right] \\
& =-\frac{1}{\alpha}\left[\beta v_{j} b b u_{i}+y_{j-1} b b u_{i}+x_{i-1} b b v_{j}\right] \\
& =-\frac{\beta}{\alpha} u_{i} b b v_{j},
\end{aligned}
$$

pois, pela hipótese de indução, $y_{j-1} b b u_{i}=x_{i-1} b b v_{j}=0$ e pelo Lema 3.1 $b u_{i} \cdot b v_{j}=0$. Temos também, da identidade (3.5) e os lemas 3.1 e 3.2, que

$$
\begin{aligned}
v_{j} b b u_{i} & =-\frac{1}{2}\left[2 b u_{i} b v_{j}+2 b b u_{i} v_{j}+2 b v_{j} b u_{i}+2 u_{i} b b v_{j}+u_{i} v_{j} b^{2}+v_{j} u_{i} b^{2}\right] \\
& =-u_{i} b b v_{j}
\end{aligned}
$$

e como $\alpha \neq \beta$, segue que

$$
u_{i} b b v_{j}=0
$$

de onde concluímos que

$$
V_{\alpha, i} \cdot b b V_{\beta, j}=\{0\}, \quad \text { para } \quad i, j \geq 1,
$$

provando o lema.

É importante notar, da demonstração anterior, que se $\alpha=\beta$, isto é $u, v \in V_{\alpha}$, então vamos ter que

$$
u b b v=-v b b u .
$$


Lembremos que por definição $W_{0,1}=\{x \in \mathcal{B} \mid a x=b x=0\}$. Queremos mostrar agora que $V_{\alpha} \cdot b b V_{\alpha} \subset W_{0,1}$. Antes de mostrar isto, é importante ressaltar que este produto, em geral, não é nulo, como mostra o seguinte exemplo.

Exemplo 3.1 Seja $\mathcal{B}=\left\langle v_{1}, v_{2}, v_{3}, v_{4}, v_{5}, v_{6}, v_{7}, v_{8}, v_{9}\right\rangle, \quad \mathcal{A}=\left\langle v_{1}, v_{2}\right\rangle, \alpha \neq 0$ e os produtos em $\mathcal{B}$, não nulos, dados por

$$
\begin{gathered}
v_{4} v_{9}=-v_{6} v_{7}=v_{3}, \quad v_{1} v_{5}=\alpha v_{4}, \quad v_{2} v_{4}=v_{5}, \quad v_{1} v_{6}=-\alpha v_{5}, \\
v_{2} v_{5}=v_{6}, \quad v_{1} v_{8}=\alpha v_{7}, \quad v_{2} v_{7}=v_{8}, \quad v_{1} v_{9}=-\alpha v_{8} \quad \text { e } v_{2} v_{8}=v_{9} .
\end{gathered}
$$

Então $\mathcal{B}$ é uma nilálgebra comutativa de potências associativas de nilíndice 4 (uma vez que é possível verificar que para todo $x \in \mathcal{B}$ cumpre-se que $x^{4}=x^{2} x^{2}=0$.)

Notamos que $\mathcal{B}$ se decompõe como $\mathcal{B}=W_{0} \oplus W_{\alpha}$, onde $W_{0}=\left\langle v_{1}, v_{2}, v_{3}\right\rangle$ e $W_{\alpha}=\left\langle v_{4}, v_{5}, v_{6}, v_{7}, v_{8}, v_{9}\right\rangle$. Da definição, é claro que

$$
0 \neq V_{\alpha} \cdot b b V_{\alpha}=\left\langle v_{3}\right\rangle \subset W_{0,1} .
$$

Lema 3.4 Se $\alpha \in F$ com $\alpha \neq 0$, então

$$
V_{\alpha} \cdot b b V_{\alpha} \subset W_{0,1} .
$$

Prova: Sejam $u \in V_{\alpha, i}$ e $v \in V_{\alpha, j}$. Então pela identidade (3.6), temos que

$$
\begin{aligned}
a u b b v=-[ & a b u b v+a(b v \cdot b u)+u a b b v+u b a b v+u(b v \cdot a b)+b a u b v \\
& +b u a b v+b(b v \cdot a u)+b v \cdot a b u+b v \cdot u a b+b v \cdot b a u] .
\end{aligned}
$$

É claro que

$$
u(b v \cdot a b)=b(b v \cdot a u)=b v \cdot u a b=b v \cdot b a u=0
$$

e pelos lemas 3.1 e 3.2 ,

$$
a b u b v=a(b v \cdot b u)=b a u b v=0 .
$$


Além disso, temos que $a b b v+b a b v=-b b a v=0$, portanto

$$
u a b b v+u b a b v=0,
$$

de onde segue que

$$
a u b b v=-[b u a b v+b v \cdot a b u] .
$$

Mas pelo Lema 3.2, $u \cdot a b v \in V_{\alpha}^{2}=\{0\}$ e $a b u \cdot b v \in V_{\alpha} \cdot b V_{\alpha}=\{0\}$, logo $a u b b v=0$. Agora pelos lemas 3.1 e 3.2 e por (3.5) temos que

$$
\begin{aligned}
b u b b v= & -\frac{1}{2}[2 b b u b v+2 b(b v \cdot b u)+2 u b b b v+2 b v \cdot b b u \\
& \left.+u\left(b v \cdot b^{2}\right)+b v \cdot u b^{2}\right] \\
= & 0 .
\end{aligned}
$$

Assim $a u b b v=b u b b v=0$, o que demonstra o lema.

Decorre, dos lemas anteriores, o seguinte resultado.

Proposição 3.5 Se $\alpha, \beta \in F$ com $\alpha \beta \neq 0$ e $\alpha \neq \beta$, então

$$
W_{\alpha} \cdot W_{\beta}=0 \quad e \quad W_{\alpha} \cdot W_{\alpha}=V_{\alpha} \cdot b b V_{\alpha} \subset W_{0,1} .
$$

O lema anterior nos diz então que para $\alpha \in F^{*}$

$$
W_{\alpha}^{2}=V_{\alpha} \cdot b b V_{\alpha} \subset W_{0,1},
$$

isto é

$$
W_{\alpha}^{2}=\left\langle u b b v, \quad \operatorname{com} u, v \in V_{\alpha} \text { e } a u b b v=b u b b v=0\right\rangle .
$$




\subsection{Os produtos $W_{\alpha}^{i} \cdot W_{\beta}^{j}$ para $\alpha, \beta \in F^{*}$}

Exploraremos agora, um pouco mais, os produtos $W_{\alpha}^{i} \cdot W_{\beta}^{j}$, para $i+j \geq 3$, lembrando que pela definicão dada no Capítulo 1 , quando $\alpha=\beta$ obtemos na verdade as potências de $W_{\alpha}$ que definimos inductivamente por

$$
W_{\alpha}^{k}=\sum_{i+j=k} W_{\alpha}^{i} \cdot W_{\alpha}^{j}
$$

\subsubsection{Produtos para $i+j=3$.}

Vejamos como é o produto $W_{\alpha}^{2} \cdot W_{\beta}$, ou seja, o produto $\left(V_{\alpha} \cdot b b V_{\alpha}\right) \cdot W_{\beta}$. Sejam $u, v \in V_{\alpha}$ e $w \in V_{\beta}$. Então pela identidade (3.3) temos

$$
u b b v \cdot b b w=-[(b u)(b b v \cdot b w)+(b b b v)(u b w)]=0 .
$$

Portanto $\left(V_{\alpha} \cdot b b V_{\alpha}\right) \cdot b b V_{\beta}=\{0\}$, isto é,

$$
W_{\alpha}^{2} \cdot b b V_{\beta}=\{0\}, \quad \text { para } \quad \alpha \beta \neq 0,
$$

e se $\alpha \neq \beta$,

$$
u b b v \cdot b w=-[b u \cdot w b b v+b b b v \cdot u w]=0,
$$

pois $w b b v \in V_{\beta} \cdot b b V_{\alpha}=0$, para $\alpha \neq \beta$. Logo

$$
W_{\alpha}^{2} \cdot b V_{\beta}=\{0\}, \quad \text { para } \quad \alpha \neq \beta \quad \text { e } \quad \alpha \beta \neq 0 .
$$

Lema 3.6 Se $\alpha, \beta \in F^{*}$, então

$$
W_{\alpha}^{2} \cdot V_{\beta}=\{0\} .
$$

Prova: Mostraremos, por indução sobre $k$, que

$$
W_{\alpha}^{2} \cdot V_{\beta, k}=\{0\}, \text { para } k \geq 1 .
$$

Para $k=1$, sejam $u, v \in V_{\alpha}$ e $w_{1} \in V_{\beta, 1}$. Então $a b w_{1}=\beta w_{1}$ e $a b b w_{1}=$ - $\beta b w_{1}$, logo por (3.3) e o Lema 3.2 temos

$$
w_{1} \cdot u b b v=\frac{1}{\beta} a b w_{1} \cdot u b b v=-\frac{1}{\beta}\left[(a u)\left(b w_{1} \cdot b b v\right)+a b b v \cdot u b w_{1}\right]=0 .
$$


Assim

$$
W_{\alpha}^{2} \cdot V_{\beta, 1}=\{0\}
$$

Seja $k>1$ e suponhamos, por hipótese de indução, que

$$
W_{\alpha}^{2} \cdot V_{\beta, t}=\{0\}
$$

para todo inteiro não negativo $t \operatorname{com} t<k$. Sejam $u, v \in V_{\alpha}$ e $w_{k} \in V_{\alpha, k}$. Então $a b w_{k}=\beta w_{k}+x_{k-1}, \quad \operatorname{com} x_{k-1} \in V_{\beta, k-1}, \quad w_{k}=\frac{1}{\beta}\left(a b w_{k}-x_{k-1}\right) \mathrm{e}$ $b w_{k}=-\frac{1}{\beta}\left(a b b w_{k}+b x_{k-1}\right)$. Logo por (3.3) e pela hipótese de indução, temos

$$
\begin{aligned}
w_{k} \cdot u b b v & =\frac{1}{\beta}\left[\left(a b w_{k}-x_{k-1}\right) \cdot u b b v\right]=\frac{1}{\beta}\left[a b w_{k} \cdot u b b v-x_{k-1} u b b v\right] \\
& =\frac{1}{\beta}\left[a b w_{k} \cdot u b b v\right]=-\frac{1}{\beta}\left[(a u)\left(b w_{k} \cdot b b v\right)+(a b b v)\left(u b w_{k}\right)\right]=0 .
\end{aligned}
$$

Assim $W_{\alpha}^{2} \cdot V_{\beta, k}=\{0\}$, para todo $k \geq 1$, isto é

$$
w \cdot u b b v=0
$$

para $w \in V_{\beta}$ e $u, v \in V_{\alpha}$, o que demonstra o lema.

Calculemos agora a potência $W_{\alpha}^{3}$, para $\alpha \in F^{*}$. Segue claramente de (3.9) e do lema anterior que $W_{\alpha}^{2} \cdot V_{\alpha}=\{0\}$ e $W_{\alpha}^{2} \cdot b b V_{\alpha}=\{0\}$, restando apenas calcular $W_{\alpha}^{2} \cdot b V_{\alpha}$. Antes disto, daremos um exemplo que mostra que este produto, em geral, não é nulo.

Exemplo 3.2 Seja $\mathcal{B}=\left\langle v_{1}, v_{2}, \ldots, v_{15}\right\rangle$ e $\mathcal{A} \subset \mathcal{B}$, uma álgebra trivial tal que $\mathcal{A}=\left\langle v_{1}, v_{2}\right\rangle$. Se $\alpha \neq 0$ e os produtos não nulos em $\mathcal{B}$, são dados por

$$
\begin{gathered}
v_{7} v_{12}=-v_{9} v_{10}=v_{3}, \quad v_{10} v_{15}=-v_{12} v_{13}=v_{4}, \quad v_{7} v_{15}=-v_{9} v_{13}=v_{5} \\
v_{3} v_{14}=v_{4} v_{8}=-v_{5} v_{11}=v_{6}, \quad v_{1} v_{8}=\alpha v_{7}, \quad v_{2} v_{7}=v_{8}, \quad v_{1} v_{9}=-\alpha v_{8}, \\
v_{2} v_{8}=v_{9}, \quad v_{1} v_{11}=\alpha v_{10}, \quad v_{2} v_{10}=v_{11}, \quad v_{1} v_{12}=-\alpha v_{11}, \quad v_{2} v_{11}=v_{12}, \\
v_{1} v_{14}=\alpha v_{13}, \quad v_{2} v_{13}=v_{14}, \quad v_{1} v_{15}=-\alpha v_{14}, \quad v_{2} v_{14}=v_{15},
\end{gathered}
$$


então $\mathcal{B}$ é uma nilálgebra comutativa de potências associativas de nilíndice 4 , pois para todo $x \in \mathcal{B}$ é possível verificar que $x^{4}=x^{2} x^{2}=0$. Uma vez que $\mathcal{A}=\left\langle v_{1}, v_{2}\right\rangle$, é claro que

$$
W_{0}=\left\langle v_{1}, v_{2}, \ldots, v_{6}\right\rangle \quad \text { e } \quad W_{\alpha}=\left\langle v_{7}, v_{8}, \ldots, v_{15}\right\rangle,
$$

tendo-se portanto a decomposição $\mathcal{B}=W_{0} \oplus W_{\alpha}$. Notemos também que

$$
W_{\alpha}^{2}=\left\langle v_{3}, v_{4}, v_{5}\right\rangle \subset W_{0,1} \quad \text { e } \quad W_{\alpha}^{2} \cdot b V_{\alpha}=\left\langle v_{6}\right\rangle \subset W_{0,1}
$$

Este exemplo, além de nos mostrar que o produto não é nulo, nos dá a idéia de que $W_{\alpha}^{2} \cdot b V_{\alpha} \subset W_{0,1}$, o que de fato acontece.

Lema 3.7 Se $\alpha \in F^{*}$, então

$$
W_{\alpha}^{3}=W_{\alpha}^{2} \cdot b V_{\alpha}=b V_{\alpha} \cdot\left(V_{\alpha} \cdot b b V_{\alpha}\right) \subset W_{0,1} .
$$

Prova: Sejam $u \in V_{\alpha, i}, \quad v \in V_{\alpha, j}$ e $w \in V_{\alpha, t}$. Então fazendo $x=a, y=$ $b w, z=u$ e $w=b b v$ na identidade (3.6) temos que

$$
\begin{aligned}
-a(b w \cdot u b b v)= & a(u(b w \cdot b b v))+a((b b v)(u \cdot b w))+(b w)(u \cdot a b b v) \\
& +(b w)(a \cdot u b b v)+(b w)(b b v \cdot a u)+u(a(b w \cdot b b v)) \\
& +u(b w \cdot a b b v)+u(b b v \cdot a b w)+b b v \cdot a u b w \\
& +(b b v)(b w \cdot a u)+b b v \cdot u a b w
\end{aligned}
$$

e como $u \cdot a b b v \in V_{\alpha} \cdot b V_{\alpha}=\{0\}, u \cdot a b w \in V_{\alpha}^{2}=\{0\}, b w \cdot a b b v \in\left(b V_{\alpha}\right)^{2}=\{0\}$ e $u(b b v \cdot a b w) \in V_{\alpha} \cdot W_{\alpha}^{2}=\{0\}$, segue que

$$
a(b w \cdot u b b v)=0 .
$$

Igualmente, fazendo $x=b, y=b w, z=u$ e $w=b b v$ em (3.6), temos

$$
\begin{aligned}
-b(b w \cdot u b b v)= & b(u(b w \cdot b b v))+b(b b v(u \cdot b w))+(b w)(u \cdot b b b v)+(b w)(b \cdot u b b v) \\
& +(b w)(b b v \cdot b u)+u(b(b w \cdot b b v))+u(b w \cdot b b b v)+u(b b v \cdot b b w) \\
& +b b v \cdot b u b w+(b b v)(b w \cdot b u)+b b v \cdot u b b w \\
= & 0
\end{aligned}
$$


pois por (3.9), $b b v \cdot u b b w \in b b V_{\alpha} \cdot W_{\alpha}^{2}=\{0\} . \operatorname{Logo}$

$$
b(b w \cdot u b b v)=0,
$$

provando o lema.

Podemos então enunciar o seguinte lema, cuja prova decorre das equações (3.9), (3.10) e dos lemas 3.6 e 3.7.

Proposição 3.8 Se $\alpha, \beta \in F$ com $\alpha \beta \neq 0$ e $\alpha \neq \beta$, então

$$
W_{\alpha}^{2} \cdot W_{\beta}=\{0\} \quad \text { e } \quad W_{\alpha}^{3}=W_{\alpha}^{2} \cdot b V_{\alpha} \subset W_{0,1} .
$$

É bom ressaltar que para $\alpha \neq 0$,

$$
W_{\alpha}^{3}=\left\langle b w \cdot u b b v, \quad \mid \quad u, v, w \in V_{\alpha}\right\rangle
$$

e para todo $u, v, w \in V_{\alpha}$, temos

$$
a(b w \cdot u b b v)=b(b w \cdot u b b v)=0 .
$$

\subsubsection{Produtos para $i+j=4$.}

Passemos agora ao caso $i+j=4$, isto é vamos calcular os produtos $W_{\alpha}^{2} \cdot W_{\beta}^{2}$ e $W_{\alpha}^{3} \cdot W_{\beta}$. Iniciaremos com o caso $\alpha \neq \beta \neq 0$.

Lema 3.9 Se $\alpha, \beta \in F^{*} \operatorname{com} \alpha \neq \beta$, então

$$
W_{\alpha}^{2} \cdot W_{\beta}^{2}=\{0\} \quad \text { e } \quad W_{\alpha}^{3} \cdot W_{\beta}=\{0\} .
$$

Prova: Sejam $u, v \in V_{\alpha}$ e $t, w \in V_{\beta}$. Então por (3.3) temos

$$
u b b v \cdot t b b w=-(u t)(b b v \cdot b b w)-u b b w \cdot t b b v=0,
$$

pois $u b b w \in W_{\alpha} \cdot W_{\beta}=\{0\} . \operatorname{Logo}$

$$
W_{\alpha}^{2} \cdot W_{\beta}^{2}=\{0\}
$$


Sejam $u, v, w \in V_{\alpha}$ e $t \in V_{\beta}$. Lembremos que os únicos produtos não nulos em $W_{\alpha}^{3}$ são os $b w \cdot u b b v$, assim temos que calcular os produtos $t(b w$. $u b b v),(b t)(b w \cdot u b b v)$ e $(b b t)(b w \cdot u b b v)$. Por $(3.3)$ temos

$$
(b t)(b w \cdot u b b v)=-(b b w)(t \cdot u b b v)-(b \cdot u b b v)(t \cdot b w)=0
$$

e

$$
(b \cdot b t)(b w \cdot u b b v)=-(b b w)(b t \cdot u b b v)-(b \cdot u b b v)(b t \cdot b w)=0,
$$

pois $b t \cdot u b b v \in b V_{\beta} \cdot W_{\alpha}^{2}=\{0\}$. Fazendo $x=t, \quad y=b w, \quad z=u$ e $w=b b v$ em (3.6) obtemos que

$$
\begin{aligned}
t(b w \cdot u b b v)= & -[t(u(b w \cdot b b v))+t(b b v \cdot u b w)+(b w)(t \cdot u b b v)+(b w)(u \cdot t b b v) \\
& +(b w)(b b v \cdot u t)+u(t(b w \cdot b b v))+u(b w \cdot t b b v)+u(b b v \cdot t b w) \\
& +(b b v)(t \cdot u b w)+b b v \cdot u t b w+(b b v)(b w \cdot u t)]=0
\end{aligned}
$$

pois $t b b v \in W_{\alpha} \cdot W_{\beta}=\{0\}$. Assim

$$
W_{\alpha}^{3} \cdot W_{\beta}=\{0\}
$$

Notemos que na demonstração anterior obtivemos que

$$
(b t)(b w \cdot u b b v)=0
$$

sem impor a condição de $\alpha$ ser diferente de $\beta$, portanto esta igualdade é válida também para todo $u, v, w, t \in V_{\alpha}$.

Passemos agora ao caso $\alpha=\beta$, isto é calculemos a potência

$$
W_{\alpha}^{4}=W_{\alpha}^{2} \cdot W_{\alpha}^{2}+W_{\alpha}^{3} \cdot W_{\alpha} .
$$

Antes de calcularmos esta potência, apresentaremos o seguinte exemplo que mostra que tal potência não é necessariamente nula. 
Exemplo 3.3 Seja $\mathcal{A}=\left\langle v_{1}, v_{2}\right\rangle, W_{0}=\left\langle v_{1}, \ldots, v_{12}\right\rangle$ e para $\alpha \neq 0, W_{\alpha}=$ $\left\langle v_{13}, \ldots, v_{24}\right\rangle$ e $W_{-\alpha}=\left\langle v_{25}, v_{26}, v_{27}\right\rangle$. Se os produtos, não nulos, entre estes elementos são definidos assim

$$
\left.\begin{array}{c}
v_{13} v_{18}=-v_{15} v_{16}=v_{3}, \quad v_{13} v_{21}=-v_{15} v_{19}=v_{4}, \\
v_{13} v_{24}=-v_{15} v_{22}=v_{5}, \quad v_{16} v_{21}=-v_{18} v_{19}=v_{6}, \\
v_{16} v_{24}=-v_{18} v_{22}=v_{7}, \quad v_{19} v_{24}=-v_{21} v_{22}=v_{8},
\end{array}\right\} \in W_{\alpha}^{2}
$$

então a álgebra $\mathcal{B}=W_{0} \oplus W_{\alpha} \oplus W_{-\alpha}$ é uma nilálgebra comutativa de p.a. de nilíndice quatro. Observamos dos produtos anteriores que

$$
W_{\alpha}^{3}=\left\langle v_{9}, v_{10}, v_{11}, v_{12}\right\rangle \subset W_{0}, \quad W_{\alpha}^{2} \cdot W_{\alpha}^{2}=\left\langle v_{26}\right\rangle \subset b V_{-\alpha} \subset W_{-\alpha}
$$

e

$$
W_{\alpha} \cdot W_{\alpha}^{3}=\left\langle v_{25}, v_{27}\right\rangle \subset V_{-\alpha}+b V_{-\alpha} \subset W_{-\alpha},
$$

portanto

$$
\{0\} \neq W_{\alpha}^{4} \subset W_{-\alpha} .
$$


Antes de enunciar o próximo lema, referente a potência $W_{\alpha}^{4}$, faremos alguns cálculos que facilitarão sua demonstração.

Sejam $u, v, w, t \in V_{\alpha}$ e $\bar{s}=u b b v$. Então pela identidade (3.6) e pelos lemas anteriores temos que

$$
\begin{aligned}
a(t(b w \cdot \bar{s}))= & -[a(b w \cdot t \bar{s})+a(\bar{s} \cdot t b w)+t(a(b w \cdot \bar{s}))+t(b w \cdot a \bar{s}) \\
& +t(\bar{s} \cdot a b w)+(b w)(a \cdot t \bar{s})+(b w)(t \cdot a \bar{s})+(b w)(\bar{s} \cdot a t) \\
& +\bar{s}(a \cdot t b w)+\bar{s}(t \cdot a b w)+\bar{s}(b w \cdot a t)], \\
a(\bar{s}(t \cdot b b w))= & -[a(t(\bar{s} \cdot b b w))+a(b b w \cdot \bar{s} t)+\bar{s}(a \cdot t b b w)+\bar{s}(t \cdot a b b w) \\
& +\bar{s}(b b w \cdot a t)+t(a(\bar{s} \cdot b b w))+t(\bar{s} \cdot a b b w)+t(b b w \cdot a \bar{s}) \\
& +(b b w)(a \cdot \bar{s} t)+(b b w)(\bar{s} \cdot a t)+(b b w)(t \cdot a \bar{s})], \\
b(\bar{s}(t \cdot b b w))= & -[b(t(\bar{s} \cdot b b w))+b(b b w \cdot \bar{s} t)+\bar{s}(b \cdot t b b w)+\bar{s}(t \cdot b b b w) \\
& +\bar{s}(b b w \cdot b t)+t(b(\bar{s} \cdot b b w))+t(\bar{s} \cdot b b b w)+t(b b w \cdot b \bar{s}) \\
& +(b b w)(b \cdot \bar{s} t)+(b b w)(\bar{s} \cdot b t)+(b b w)(t \cdot b \bar{s})]
\end{aligned}
$$

e

$$
\begin{aligned}
\bar{s} \cdot t b b w= & -[\bar{s}(b \cdot t b w)+\bar{s}(b w \cdot b t)+t(\bar{s} \cdot b b w)+t(b(\bar{s} \cdot b w)) \\
& +t(b w \cdot b \bar{s})+(b w)(\bar{s} \cdot b t)+(b w)(t \cdot b \bar{s})+(b w)(b \cdot t \bar{s}) \\
& +b(\bar{s} \cdot t b w)+b(t(b w \cdot \bar{s}))+b(b w \cdot t \bar{s})] .
\end{aligned}
$$

Uma vez que $\bar{s} \in W_{\alpha}^{2} \subset W_{0,1}$, temos que $a \bar{s}=b \bar{s}=0$. Também temos por (3.9) e pelo Lema 3.6 que $\bar{s} \cdot t=\bar{s} \cdot a b w=\bar{s} \cdot b b w=0$, pois $a b w \in V_{\alpha}$.

Assim $a(t(b w \cdot \bar{s}))=-t(a(b w \cdot \bar{s})), a(\bar{s} \cdot t b b w)=-t(\bar{s} \cdot a b b w), b(\bar{s} \cdot t b b w)=$ $-(b b w)(b t \cdot \bar{s})$ e $\bar{s} \cdot t b b w=-t(b(b w \cdot \bar{s}))-(b w)(b t \cdot \bar{s})-b(t(b w \cdot \bar{s}))$. E novamente de $b w \cdot \bar{s} \in W_{\alpha}^{3} \subset W_{0,1}$, segue que $a(b w \cdot \bar{s})=b(b w \cdot \bar{s})=0$ e de (3.11) 
$(b w)(b t \cdot \bar{s})=0 . \quad \log O$

$$
\begin{aligned}
a(t(b w \cdot \bar{s})) & =0 \\
a(\bar{s} \cdot t b b w) & =-t(a b b w \cdot \bar{s}) \\
b(\bar{s} \cdot t b b w) & =(-b b w)(b t \cdot \bar{s}) \\
\bar{s} \cdot t b b w & =-b(t(b w \cdot \bar{s}))
\end{aligned}
$$

Com estas igualdades podemos então mostrar o seguinte lema.

Lema 3.10 Se $\alpha \in F^{*}$, então

$$
\begin{gathered}
V_{\alpha} \cdot W_{\alpha}^{3} \subset V_{-\alpha}, \quad b V_{\alpha} \cdot W_{\alpha}^{3}=\{0\}, \quad b b V_{\alpha} \cdot W_{\alpha}^{3} \subset b b V_{-\alpha} \quad e \\
W_{\alpha}^{2} \cdot W_{\alpha}^{2} \subset b V_{-\alpha} .
\end{gathered}
$$

Prova: De (3.11) é claro que $b V_{\alpha} \cdot W_{\alpha}^{3}=\{0\}$.

Para os outros produtos sejam $u, v, w, t \in V_{\alpha}$. Vejamos primeiro que $V_{\alpha} \cdot W_{\alpha}^{3} \subset V_{-\alpha}$, isto é que $t(b w \cdot u b b v) \in V_{-\alpha}$. Mostraremos, por indução sobre $i$, que se $w_{i} \in V_{\alpha, i}$, então

$$
t\left(b w_{i} \cdot u b b v\right) \in V_{-\alpha, i}, \quad \text { para } \quad i \geq 1 .
$$

Notemos primeiramente que por (3.12)

$$
a\left(t\left(b w_{i} \cdot u b b v\right)\right)=0
$$

e por (3.13) e (3.15)

$$
u b b v \cdot t b b w_{i}=-b\left(t\left(b w_{i} \cdot u b b v\right)\right) \quad \text { e } \quad a\left(u b b v \cdot t b b w_{i}\right)=-t\left(a b b w_{i} \cdot u b b v\right)
$$

$\log 0$

$$
a\left(b\left(t\left(b w_{i} \cdot u b b v\right)\right)\right)=t\left(a b b w_{i} \cdot u b b v\right) .
$$

Para $i=1$, seja $w_{1} \in V_{\alpha, 1}$. Então $a b w_{1}=\alpha w_{1}$ e $a b b w_{1}=-\alpha b w_{1}, \log 0$

$$
a\left(b\left(t\left(b w_{1} \cdot u b b v\right)\right)\right)=t\left(a b b w_{1} \cdot u b b v\right)=-\alpha t\left(b w_{1} \cdot u b b v\right)
$$


e assim

$$
a\left(t\left(b w_{1} \cdot u b b v\right)\right)=0 \quad \text { e } \quad a\left(b\left(t\left(b w_{1} \cdot u b b v\right)\right)\right)=-\alpha t\left(b w_{1} \cdot u b b v\right) .
$$

Portanto

$$
t\left(b w_{1} \cdot u b b v\right) \in V_{-\alpha, 1} .
$$

Suponhamos, por hipótese de indução, que se $w_{k} \in V_{\alpha, k}$, então

$$
t\left(b w_{k} \cdot u b b v\right) \in V_{-\alpha, k} \quad \text { para } \quad 1 \leq k \leq i-1
$$

e verifiquemos a relação para $k=i$.

Seja $w_{i} \in V_{\alpha, i}$. Então $a b w_{i}=\alpha w_{i}+x_{i-1} \operatorname{com} x_{i-1} \in V_{\alpha, i-1}$, e portanto $a b b w_{i}=-\alpha b w_{i}-b x_{i-1}$. Assim

$$
a\left(b\left(t\left(b w_{i} \cdot u b b v\right)\right)\right)=t\left(a b b w_{i} \cdot u b b v\right)=-\alpha t\left(b w_{i} \cdot u b b v\right)-t\left(b x_{i-1} \cdot u b b v\right) .
$$

Uma vez que pela hipótese de indução temos $y_{i-1}=-t\left(b x_{i-1} \cdot u b b v\right) \in$ $V_{-\alpha, i-1}$, segue que

$$
a\left(t\left(b w_{i} \cdot u b b v\right)\right)=0 \quad \text { e } \quad a\left(b\left(t\left(b w_{i} \cdot u b b v\right)\right)\right)=-\alpha t\left(b w_{i} \cdot u b b v\right)+y_{i-1}
$$

com $y_{i-1} \in V_{-\alpha, i-1}$. Portanto

$$
t\left(b w_{i} \cdot u b b v\right) \in V_{-\alpha, i}, \quad \text { para } \quad i \geq 1
$$

o que mostra que

$$
t(b w \cdot u b b v) \in V_{-\alpha} .
$$

Decorre da parte anterior e de (3.15) que

$$
u b b v \cdot t b b w \in b V_{-\alpha},
$$

ou seja,

$$
V_{\alpha} \cdot W_{\alpha}^{3} \subset V_{-\alpha} \quad \text { e } \quad W_{\alpha}^{2} \cdot W_{\alpha}^{2} \subset b V_{-\alpha} .
$$

Temos também por (3.14) e (3.15) que

$$
(b b w)(b t \cdot u b b v)=-b(u b b v \cdot t b b w)=b(b(t(b w \cdot u b b v)))
$$


isto é

$$
(b b w)(b t \cdot u b b v)=b(b(t(b w \cdot u b b v))) \in b b V_{-\alpha} .
$$

Portanto

$$
b b V_{\alpha} \cdot W_{\alpha}^{3} \subset b b V_{-\alpha}
$$

Temos então do lema anterior que

$$
W_{\alpha}^{4} \subset W_{-\alpha}
$$

isto é

$$
W_{\alpha}^{4}=\left\langle r, b r, b b r, \quad \text { com } r \in V_{-\alpha} \text { e } r=t(b w \cdot u b b v) \quad \text { com } t, w, u, v \in V_{\alpha}\right\rangle
$$

Proposição 3.11 Se $\alpha, \beta \in F^{*} \operatorname{com} \alpha \neq \beta$, então para $i+j=4$

$$
W_{\alpha}^{i} \cdot W_{\beta}^{j}=\{0\} \quad \text { e } \quad W_{\alpha}^{4} \subset W_{-\alpha} .
$$

\subsubsection{Produtos para $i+j=5$.}

Continuando com o cálculo dos produtos $W_{\alpha}^{i} \cdot W_{\beta}^{j}$, passemos ao caso $i+j=5$ lembrando que neste caso temos os produtos

$$
W_{\alpha}^{4} \cdot W_{\beta} \quad \text { e } \quad W_{\alpha}^{2} \cdot W_{\beta}^{3}
$$

Lema 3.12 Sejam $\alpha, \beta \in F^{*}$. Então

$$
W_{\alpha}^{2} \cdot W_{\beta}^{3}=\{0\} \quad \text { e } \quad W_{\alpha}^{4} \cdot W_{\beta}=\{0\} .
$$

Prova: Sejam $u, v \in V_{\alpha}$ e $w, t, s \in V_{\beta}$. Então por (3.3) $(u b b v)(b w \cdot t b b s)=-(u \cdot b w)(b b v \cdot t b b s)-(u \cdot t b b s)(b b v \cdot b w)=0$ 
$\log \mathrm{O}$

$$
W_{\alpha}^{2} \cdot W_{\beta}^{3}=\{0\}
$$

Como $W_{\alpha}^{4} \subset W_{-\alpha}$, segue que $W_{\alpha}^{4} \cdot W_{\beta} \subset W_{-\alpha} \cdot W_{\beta}$. Portanto se $\beta \neq-\alpha$, então $W_{\alpha}^{4} \cdot W_{\alpha}=\{0\}$ e se $\beta=-\alpha$, então vamos ter que $W_{\alpha}^{4} \cdot W_{\beta} \subset W_{-\alpha}^{2}$, cujos únicos produtos não nulos são os $u b b v \operatorname{com} u, v \in V_{-\alpha}$, mas neste caso $u \in V_{-\alpha} \cap W_{\alpha}^{4}$, portanto $u=t(b w \cdot r b b s)$ com $t, w, r, s \in V_{\alpha}$. Logo $u b b v=(t(b w \cdot r b b s))(b b v)$ e por $(3.3)$

$$
(t(b w \cdot r b b s))(b b v)=-(b t)(b w \cdot b v)-(t \cdot b v)(b(b w \cdot r b b s))=0,
$$

e desta forma

$$
W_{\alpha}^{4} \cdot W_{\beta}=\{0\}
$$

Como consequência imediata do lema anterior temos que para $\alpha \in F^{*}$

$$
W_{\alpha}^{5}=\{0\}
$$

\subsubsection{Produtos para $i+j \geq 6$.}

Notemos que para $\alpha, \beta \in F^{*}$ e $i, j \geq 5, W_{\alpha}^{i} \cdot W_{\beta}^{j}=\{0\}$, portanto a igualdade anterior é valida também se $i+j \geq 9$, restando então calcular o produto para $6 \leq i+j \leq 8$.

Lema 3.13 Sejam $\alpha, \beta \in F^{*}$. Então

$$
W_{\alpha}^{3} \cdot W_{\beta}^{3}=\{0\} \quad \text { e } \quad W_{\alpha}^{4} \cdot W_{\beta}^{2}=\{0\} .
$$

Prova: Sejam $u, v, w \in V_{\alpha}$ e $r, s, t \in V_{\beta}$. Então por (3.3)

$$
(b w \cdot u b b v)(b t \cdot r b b s)=-(b w \cdot b t)(u b b v \cdot r b b s)-(b t \cdot u b b v)(b w \cdot r b b s),
$$

isto é,

$$
(b w \cdot u b b v)(b t \cdot r b b s)=(b t \cdot u b b v)(b w \cdot r b b s) .
$$


Se $\alpha \neq \beta$, então $b t \cdot u b b v \in W_{\beta} \cdot W_{\alpha}^{2}=\{0\}$ e se $\alpha=\beta$, então $b t \cdot u b b v \in W_{\alpha}^{3}$. Seja $\alpha=\beta$ e $\bar{p}=b t \cdot u b b v$. Então $(b t \cdot u b b v)(b w \cdot r b b s)=\bar{p}((b w)(r \cdot b b s))$ e por (3.6) e os lemas anteriores temos

$$
\begin{aligned}
\bar{p}((b w)(r \cdot b b s))= & -[\bar{p}(r(b w \cdot b b s))+\bar{p}(b b s \cdot r b w)+(b w)(\bar{p} \cdot r b b s)+ \\
& (b w)(r(\bar{p} \cdot b b s))+(b w)(b b s \cdot \bar{p} r)+r(\bar{p}(b w \cdot b b s))+ \\
& r((b w)(\bar{p} \cdot b b s))+r(b b s \bar{p} \cdot b w)+(b b s)(\bar{p} \cdot r b w) \\
& +(b b s)(b w \cdot \bar{p} r)+(b b s)(r(\bar{p} \cdot b w))] \\
= & -[b w(\bar{p} \cdot r b b s)+(b w)(r(\bar{p} \cdot b b s))+(b w)(b b s \cdot \bar{p} r)+ \\
& r((b w)(\bar{p} \cdot b b s))+r(b b s(\bar{p} \cdot b w))+(b b s)(b w \cdot \bar{p} r)+ \\
& (b b s)(r(\bar{p} \cdot b w))] .
\end{aligned}
$$

Como $\bar{p} \cdot b b s, \bar{p} r, \bar{p} \cdot b w \in W_{\alpha}^{3} \cdot W_{\alpha} \subset W_{-\alpha}$, temos que $r(\bar{p} \cdot b b s), b w(\bar{p} \cdot b b s), b b s \cdot$ $\bar{p} r, b w \cdot \bar{p} r, r(\bar{p} \cdot b w),(b b s)(\bar{p} \cdot b w) \in W_{\alpha} \cdot W_{-\alpha}=\{0\}$ e $\bar{p} \cdot r b b s \in W_{\alpha}^{3} \cdot W_{\alpha}^{2} \subset$ $W_{\alpha}^{5}=\{0\} . \operatorname{Logo} \bar{p}(b w \cdot r b b s)=0$, o que mostra que $W_{\alpha}^{3} \cdot W_{\alpha}^{3}=\{0\}$ e portanto

$$
W_{\alpha}^{3} \cdot W_{\beta}^{3}=\{0\} .
$$

Uma vez que $W_{\alpha}^{4} \cdot W_{\beta}^{2} \subset W_{-\alpha} \cdot W_{\beta}^{2}$ temos que analisar apenas o caso $\beta=-\alpha$, pois se $\beta \neq-\alpha$ é claro que $W_{\alpha}^{4} \cdot W_{\beta}^{2}=\{0\}$. Lembrando que os únicos produtos não nulos de $W_{-\alpha}^{3}$ são $b w \cdot u b b v$, temos que calcular apenas este produto, mas neste caso temos que $w \in V_{-\alpha} \cap W_{\alpha}^{4}$. Logo $w=t(b q \cdot r b b s)$ e segue de (3.3) e (3.16) que

$$
b w \cdot u b b v=-(b u)(w \cdot b b v)-(b b b v)(w \cdot u)=-(b u)[(t(b q \cdot r b b s))(b b v)]=0 .
$$

Assim

$$
W_{\alpha}^{4} \cdot W_{\beta}^{2}=\{0\}
$$

Para o caso $i+j=7$, temos que estudar somente o produto $W_{\alpha}^{3} \cdot W_{\beta}^{4}$. Devido ao fato de $W_{\alpha}^{3} \cdot W_{\beta}^{4} \subset W_{\alpha}^{3} \cdot W_{-\beta}$, temos que nos preocupar apenas com o caso $\beta=-\alpha$, pois se $\beta \neq-\alpha$, temos que $W_{\alpha}^{3} \cdot W_{-\beta}=\{0\}$ e portanto $W_{\alpha}^{3} \cdot W_{\beta}^{4}=\{0\}$. 
Lema 3.14 Sejam $\alpha, \beta \in F^{*}$. Então

$$
W_{\alpha}^{3} \cdot W_{\beta}^{4}=\{0\}
$$

Prova: Seja $\beta=-\alpha$. Queremos calcular o produto $W_{\alpha}^{3} \cdot W_{-\alpha}^{4} \subset W_{\alpha}^{4}$ e dado que os elementos de $W_{\alpha}^{3}$ são da forma $b w \cdot u b b v$, com $w, u, v \in V_{\alpha}$ e $W_{-\alpha}^{4} \subset W_{\alpha}$, temos de calcular os produtos $r(b w \cdot u b b v),(b r)(b w \cdot u b b v) \mathrm{e}$ $(b b r)(b w \cdot u b b v)$, com $r \in V_{\alpha} \cap W_{-\alpha}^{4}$. Lembremos que por (3.11) $(b r)(b w$. $u b b v)=0$, restando calcular os outros dois produtos. Como $r=t(b s \cdot p b b q)$, com $t, s, p, q \in V_{-\alpha}$, temos por (3.3) que

$$
\begin{aligned}
r(b w \cdot u b b v) & =(t(b s \cdot p b b q))(b w \cdot u b b v) \\
& =-(t \cdot b w)((u b b v)(b s \cdot p b b q))-(t \cdot u b b v)((b w)(b s \cdot p b b q))=0
\end{aligned}
$$

e

$$
(b b r)(b w \cdot u b b v)=-(b \cdot b w)(b r \cdot u b b v)-(b \cdot u b b v)(b r \cdot b w)=0,
$$

pois $b r \cdot u b b v \in W_{-\alpha}^{4} \cdot W_{\alpha}^{2}=\{0\}$. Assim $W_{\alpha}^{3} \cdot W_{-\alpha}^{4}=\{0\}$ e do comentário anterior ao lema tínhamos que $W_{\alpha}^{3} \cdot W_{\beta}^{4}=\{0\}$ para $\beta \neq-\alpha$. Em decorrência disto, temos

$$
W_{\alpha}^{3} \cdot W_{\beta}^{4}=\{0\},
$$

para todo $\alpha, \beta \in F^{*}$, ficando provado o lema.

Segue imediatamente do lema anterior que

$$
W_{\alpha}^{7}=\{0\}
$$

Chegamos finalmente ao caso $i+j=8$ para o qual devemos calcular somente o produto $W_{\alpha}^{4} \cdot W_{\beta}^{4}$, notando que se $\alpha \neq \beta$, então $W_{\alpha}^{4} \cdot W_{\beta}^{4} \subset W_{-\alpha}$. $W_{-\beta}=\{0\}$. Portanto analisemos apenas o caso $\alpha=\beta$, isto é $W_{\alpha}^{4} \cdot W_{\alpha}^{4} \subset$ $W_{-\alpha}^{2}$. Mas os únicos produtos não nulos de $W_{-\alpha}^{2}$ são $u b b v$, com $u, v \in V_{-\alpha}$, e como neste caso $u=t(b r \cdot p b b q)$, com $t, r, p, q \in V_{\alpha}$ segue de (3.3) e (3.11) que

$u b b v=(t(b r \cdot p b b q))(b b v)=(b t)((b v)(b r \cdot p b b q))-(t \cdot b v)(b(b r \cdot p b b q))=0$. 
Portanto $W_{\alpha}^{4} \cdot W_{\alpha}^{4}=\{0\}$, de onde podemos concluir que

$$
W_{\alpha}^{4} \cdot W_{\beta}^{4}=\{0\} \quad \text { e } \quad W_{\alpha}^{8}=\{0\} .
$$

Lema 3.15 Sejam $\alpha, \beta \in F^{*}$. Então

$$
W_{\alpha}^{4} \cdot W_{\beta}^{4}=\{0\}
$$

Podemos agora, utilizando todos os resultados anteriores, enunciar os seguintes dois teoremas

Teorema 3.16 Se $\alpha \in F^{*}$, então

$$
W_{\alpha}^{k}=\{0\}, \quad \text { para todo } \quad k \geq 5 \text {. }
$$

Prova: Para $k \geq 5$,

$$
W_{\alpha}^{k}=\sum_{5 \leq i+j \leq k} W_{\alpha}^{i} \cdot W_{\alpha}^{j}=\sum_{i, j \leq 4} W_{\alpha}^{i} \cdot W_{\alpha}^{j}=\sum_{5 \leq i+j \leq 8} W_{\alpha}^{i} \cdot W_{\alpha}^{j}=0 .
$$

Teorema 3.17 Sejam $\alpha, \beta \in F^{*}$, com $\alpha \neq \beta$. Então

$$
W_{\alpha}^{i} \cdot W_{\beta}^{j}=\{0\}, \quad \text { para } \quad i, j \geq 1
$$

Com isto caracterizamos os produtos $W_{\alpha}^{i} \cdot W_{\beta}^{j}$ sempre que $\alpha, \beta \in F^{*}$.

\subsection{O produto $W_{0} \cdot W_{\alpha}$ para $\alpha \in F$.}

Gostaríamos de caracterizar os produtos $W_{\alpha}^{i} \cdot W_{\beta}^{j}$, da mesma forma como fizemos na seção anterior, para o caso em que um dos dois, ou ambos escalares, são nulos, isto é caracterizar os produtos $W_{\alpha}^{i} \cdot W_{0}^{j}$ e $W_{0}^{k}$, lembrando que para $l \geq 1, W_{0, l}=\left\{w \in \mathcal{B}: a w, b w \in W_{0, l-1}\right\}$ e $W_{0}=\bigcup_{k=0}^{n} W_{0, k}$. Mas no 
caso mais simples, isto é, para $i, j=1$ e $k=2$ não temos o mesmo comportamento como no caso dos escalares não nulos. No que segue apresentaremos quatro exemplos, os quais nos mostram que os produtos

$$
W_{0} \cdot W_{0} \quad \text { e } \quad W_{0} \cdot W_{\alpha}
$$

são arbitrários.

Exemplo 3.4 Seja $\mathcal{B}=\left\langle v_{1}, v_{2}, v_{3}, v_{4}, v_{5}, v_{6}\right\rangle$ com produtos não nulos dados por

$$
v_{1} v_{5}=\alpha v_{4}, \quad v_{2} v_{4}=v_{3}^{2}=v_{5}, \quad v_{1} v_{6}=-\alpha v_{5} \quad \text { e } \quad v_{2} v_{5}=v_{6},
$$

com $\alpha \neq 0$. Então se escolhermos $\mathcal{A} \subset \mathcal{B}$ como $\mathcal{A}=\left\langle v_{1}, v_{2}\right\rangle$, temos que $\mathcal{B}$ é uma nilálgebra comutativa de potências associativas de nilíndice 4 com a decomposição $\mathcal{B}=W_{0} \oplus W_{\alpha}$, onde $W_{0}=\left\langle v_{1}, v_{2}, v_{3}\right\rangle$ e $W_{\alpha}=\left\langle v_{4}, v_{5}, v_{6}\right\rangle$. Desta forma,

$$
W_{0} \cdot W_{0} \subset W_{\alpha} \quad \text { e } \quad W_{0} \cdot W_{\alpha}=W_{\alpha} .
$$

Convém ressaltar que $\mathcal{B}$ não é nilpotente, pois $\mathcal{B}^{r}=\mathcal{B}^{2} \neq\{0\}$, para $r \geq 3$, mas é solúvel, de índice de solubilidade 2 , uma vez que $\mathcal{B}^{2}=W_{\alpha}$ e $W_{\alpha}^{2}=$ $\{0\}$, isto é, $\mathcal{B}^{(2)}=\{0\}$.

No exemplo anterior vimos que para $\alpha \in F^{*}$, os produtos $W_{\alpha} \cdot W_{0}$ e $W_{0}^{2}$ estavam contidos em $W_{\alpha}$, isto nem sempre ocorre, como mostram os seguinte exemplos.

Exemplo 3.5 Seja $\mathcal{B}=\left\langle v_{1}, v_{2}, \ldots, v_{8}\right\rangle$ e $\mathcal{A} \subset \mathcal{B}$ uma álgebra trivial tal que $\mathcal{A}=\left\langle v_{1}, v_{2}\right\rangle$ e os produtos em $\mathcal{B}$, não nulos, são dados por

$$
v_{3}^{2}=v_{4} v_{7}=v_{5}, \quad v_{1} v_{7}=\alpha v_{6}, \quad v_{2} v_{6}=v_{7}, \quad v_{1} v_{8}=-\alpha v_{7} \quad \text { e } \quad v_{2} v_{7}=v_{8},
$$

com $\alpha \neq 0$. Então $\mathcal{B}$ é uma nilálgebra comutativa de potências associativas de nilíndice 4 e $\mathcal{B}=W_{0} \oplus W_{\alpha}$, onde $W_{0}=\left\langle v_{1}, v_{2}, v_{3}, v_{4}, v_{5}\right\rangle$ e $W_{\alpha}=$ $\left\langle v_{6}, v_{7}, v_{8}\right\rangle$. Assim

$$
W_{0} \cdot W_{0} \subset W_{0} \quad \text { e } \quad W_{0} \cdot W_{\alpha} \subset W_{0} \oplus W_{\alpha} .
$$


Observamos que $\mathcal{B}^{2}=\left\langle v_{5}, v_{6}, v_{7}, v_{8}\right\rangle$. Portanto $\mathcal{B}^{2} \mathcal{B}^{2}=0$ o que mostra que $\mathcal{B}$ é solúvel de índice 2 , mas não é nilpotente, pois $\mathcal{B}^{r}=\mathcal{B}^{2}$, para todo $r \geq 3$.

Uma outra idéia, que poderíamos ter, é a de que sempre o produto $W_{0} \cdot W_{\alpha}$ está inteiramente contido na soma $W_{0} \oplus W_{\alpha}$. Isto não é verdade, uma vez que podem existir $\beta_{1}, \ldots, \beta_{i} \in F$, todos não nulos e diferentes de $\alpha$, tais que uma parte do produto $W_{0} \cdot W_{\alpha}$ esteja contido na $\bigoplus W_{\beta_{i}}$. O seguinte exemplo ilustra este caso.

Exemplo 3.6 Seja $\mathcal{B}=\left\langle v_{1}, v_{2}, v_{3}, \ldots, v_{11}\right\rangle$. Se $\mathcal{A}=\left\langle v_{1}, v_{2}\right\rangle$ e os produtos em $\mathcal{B}$, não nulos, são dados por

$$
\begin{gathered}
v_{3} v_{7}=v_{4}, \quad v_{1} v_{7}=\alpha v_{6}, \quad v_{2} v_{6}=v_{7}, \quad v_{1} v_{8}=-\alpha v_{7}, \quad v_{2} v_{7}=v_{8}, \quad v_{5} v_{7}=v_{9}, \\
v_{1} v_{10}=\beta v_{9}, \quad v_{2} v_{9}=-v_{5} v_{8}=v_{10}, \quad v_{1} v_{11}=-\beta v_{10}, \quad v_{2} v_{10}=v_{11},
\end{gathered}
$$

com $\alpha \neq \beta \neq 0$. Então $W_{0}=\left\langle v_{1}, v_{2}, v_{3}, v_{4}, v_{5}\right\rangle, W_{\alpha}=\left\langle v_{6}, v_{7}, v_{8}\right\rangle$ e $W_{\beta}=$ $\left\langle v_{9}, v_{10}, v_{11}\right\rangle$. Desta forma temos que $\mathcal{B}=W_{0} \oplus W_{\alpha} \oplus W_{\beta}$ é uma nilálgebra comutativa de potências associativas de nilíndice 4 e

$$
W_{0} \cdot W_{\alpha} \subset W_{0} \oplus W_{\alpha} \oplus W_{\beta}
$$

Ao calcular as potências para $\mathcal{B}$ achamos que $\mathcal{B}^{2}=\left\langle v_{4}, v_{6}, v_{7}, v_{8}, v_{9}, v_{10}, v_{11}\right\rangle$ e $\mathcal{B}^{3}=\left\langle v_{6}, v_{7}, v_{8}, v_{9}, v_{10}, v_{11}\right\rangle$, tendo-se então que

$$
\mathcal{B}^{m}=\left\{\begin{array}{l}
\mathcal{B}^{2}, \text { se } n \text { par } \geq 4 \\
\mathcal{B}^{3}, \text { se } n \text { ímpar } \geq 5,
\end{array}\right.
$$

o que nos mostra que $\mathcal{B}$ é solúvel de índice 2, mas não é nilpotente.

Finalizamos esta seção com o seguinte exemplo. 
Exemplo 3.7 Seja $\mathcal{B}=\left\langle v_{1}, v_{2}, v_{3}, v_{4}, v_{5}, v_{6}, v_{7}, v_{8}, v_{9}, v_{10}, v_{11}\right\rangle$ e $\mathcal{A} \subset \mathcal{B}$, uma álgebra trivial tal que $\mathcal{A}=\left\langle v_{1}, v_{2}\right\rangle$ e os produtos em $\mathcal{B}$, não nulos, são dados por:

$$
\begin{gathered}
v_{1} v_{5}=v_{4}, \quad v_{1} v_{7}=\alpha v_{6}, \quad v_{2} v_{6}=v_{3}^{2}=v_{7}, \quad v_{1} v_{8}=-\alpha v_{7}, \quad v_{2} v_{7}=v_{8}, \\
v_{1} v_{10}=\beta v_{9}, \quad v_{2} v_{9}=v_{5}^{2}=v_{10}, \quad v_{1} v_{11}=-\beta v_{10} \quad \text { e } \quad v_{2} v_{10}=v_{11},
\end{gathered}
$$

$\operatorname{com} \alpha \neq \beta \neq 0$. Então $\mathcal{B}$ é uma nilálgebra comutativa de potências associativas de nilíndice 4 e $\mathcal{B}=W_{0} \oplus W_{\alpha} \oplus W_{\beta}$, onde $W_{0}=\left\langle v_{1}, v_{2}, v_{3}, v_{4}, v_{5}\right\rangle$, $W_{\alpha}=\left\langle v_{6}, v_{7}, v_{8}\right\rangle$ e $W_{\beta}=\left\langle v_{9}, v_{10}, v_{11}\right\rangle$. Assim

$$
W_{0} \cdot W_{0} \subset W_{0} \oplus W_{\alpha} \oplus W_{\beta}
$$

Notemos que $\mathcal{B}^{2}=\left\langle v_{4}, v_{6}, v_{7}, v_{8}, v_{9}, v_{10}, v_{11}\right\rangle$ e $\mathcal{B}^{3}=\left\langle v_{6}, v_{7}, v_{8}, v_{9}, v_{10}, v_{11}\right\rangle$ de onde segue que $\mathcal{B}^{2} \mathcal{B}^{2}=0$ e $\mathcal{B}^{r}=\mathcal{B}^{3}$ para $r \geq 4$, o que nos mostra que $\mathcal{B}$ é solúvel de índice 2 , mas não é nilpotente.

Os exemplos anteriores nos fornecem as possíveis inclusões para $W_{0}^{2}$ e $W_{0} \cdot W_{\alpha}$ quando $\alpha \neq 0$, isto é

$$
W_{0} \cdot W_{0} \subset\left\{\begin{array} { l } 
{ W _ { 0 } } \\
{ W _ { \alpha } } \\
{ W _ { 0 } \oplus W _ { \alpha } \oplus W _ { \beta } }
\end{array} \quad \text { e } \quad W _ { 0 } \cdot W _ { \alpha } \subset \left\{\begin{array}{l}
W_{\alpha} \\
W_{0} \oplus W_{\alpha} \\
W_{0} \oplus W_{\alpha} \oplus W_{\beta}
\end{array}\right.\right.
$$

sendo que $\beta \in F^{*}$ e $\alpha \neq \beta$. É claro que a única inclusão que não é possível é $W_{0} \cdot W_{\alpha} \subset W_{0}$.

\subsection{Algumas nilálgebras que não são simples.}

Utilizando alguns dos resultados anteriores, caracterizaremos duas classes de álgebras que não são simples. Lembremos que estamos trabalhando com o fato de $\mathcal{B}$ ser uma nilálgebra comutativa de potências associativas de dimensão $n$ e nilíndice 4 , que se decompõe como $\mathcal{B}=\bigoplus_{\alpha \in F} W_{\alpha}$. 
Tínhamos no Lema 3.5 que $W_{\alpha} \cdot W_{\alpha} \subset W_{0,1}$. Mostraremos que

$$
W_{\alpha} \cdot W_{\alpha} \not \subset \mathcal{A} \text {. }
$$

Se $W_{\alpha}^{2} \subset \mathcal{A}$, então existem $u, v \in V_{\alpha}$, tal que $u b b v \in \mathcal{A}$, isto é $u b b v=$ $\lambda_{1} a+\lambda_{2} b$. Por (3.9), $0=b b u \cdot u b b v=\lambda_{1} a b b u+\lambda_{2} b b b u=\lambda_{1} a b b u$ e como $a b b u \neq 0$, segue que $\lambda_{1}=0$, portanto $u b b v=\lambda_{2} b$.

Pelo Lema 3.6, temos que $0=u \cdot u b b v=\lambda_{2} b u$ e dado que $b u \neq 0$, segue que $\lambda_{2}=0, \operatorname{logo} u b b v=0$. Portanto se $W_{0,1}=\mathcal{A}$, então para $\alpha \in F^{*}$, $W_{\alpha}^{2}=\{0\}$.

Como uma consequência dos fatos anteriores temos o seguinte resultado, que nos mostra que não é possível (neste contexto) construir um contraexemplo para a conjectura de Albert, se $W_{0}=\mathcal{A}$, isto é, a dimensão de $W_{0}$ é dois.

Teorema 3.18 Não existem nilálgebras comutativas de potências associativas de nilíndice 4, simples, com a seguinte forma

$$
\mathcal{B}=\mathcal{A} \oplus\left(\bigoplus_{0 \neq \alpha \in F} W_{\alpha}\right)
$$

Prova: Sabemos que $\mathcal{A} \cdot W_{\alpha} \subset W_{\alpha}$ e $W_{\alpha} \cdot W_{\beta}=\{0\}$ para $\alpha, \beta \in F^{*}$ e $\alpha \neq \beta$. Neste caso $W_{\alpha}^{2}=\{0\}$ para todo $\alpha \in F^{*}$, portanto temos que $\mathcal{B}^{2} \subset \oplus_{\alpha \neq 0} W_{\alpha}$, o que mostra que $\mathcal{B}^{2} \neq \mathcal{B}$, isto é $\mathcal{B}$ não é simples.

O seguinte lema nos diz que, se $W_{0}$ tem dimensão 3 e existe $\alpha \in F^{*}$ tal que $W_{\alpha} \neq 0$, então $W_{0}=W_{0,1}$.

Lema 3.19 Seja $W_{0}=\mathcal{A} \oplus\langle w\rangle \quad$ e $\alpha \in F^{*}$ tal que $W_{\alpha} \neq 0$. Então $\mathcal{A} w=\{0\}$.

Prova: Como $W_{0}$ é um $\mathcal{A}$-módulo, sabemos que $a w, b w \in W_{0}$. Seja $a w=$ $\lambda_{1} a+\lambda_{2} b+\lambda_{3} w$, então da nilpotência do operador $L_{a}$ segue que $0=L_{a}^{3}(w)=$ 
$\lambda_{3}^{2} a w$, de onde concluímos que $\lambda_{3}=0$. Assim $a w=\lambda_{1} a+\lambda_{2} b$ e analogamente $b w=\gamma_{1} a+\gamma_{2} b$, ou seja,

$$
a w=\lambda_{1} a+\lambda_{2} b \quad \text { e } \quad b w=\gamma_{1} a+\gamma_{2} b .
$$

Seja $u \in V_{\alpha .}$ então por (3.3),

$$
b u \cdot a w=-(b a)(u w)-(b w)(a u)=0,
$$

$\operatorname{logo} b u \cdot a w=\lambda_{1} a b u+\lambda_{2} b b u=0$ e portanto $\lambda_{1}=\lambda_{2}=0$. Da mesma forma por (3.3) temos

$$
b u \cdot b w=-\frac{1}{2} b^{2}(u w)=0 .
$$

Desta forma $b u \cdot b w=\gamma_{1} a b u+\gamma_{2} b b u=0$, de onde segue que $\gamma_{1}=\gamma_{2}=0$. Assim $a w=b w=0$, isto é

$$
w \mathcal{A}=\{0\}
$$

Antes de passarmos ao próximo lema, calcularemos um outro produto, que nos será útil na próxima demonstração.

Sejam $w \in \mathcal{B}$, e $u, v \in V_{\alpha}$. Então

$$
u \cdot w v=0 .
$$

Mostraremos, por indução sobre $i$, que para $u_{i} \in V_{\alpha, i}$,

$$
u_{i} \cdot w v=0, \quad \text { para } \quad i \geq 1
$$

Para $i=1$, seja $u_{1} \in V_{\alpha, 1}$. Então por (3.3) segue

$$
u_{1} \cdot w v=\frac{1}{\alpha}\left(a b u_{1} \cdot w v\right)=-\frac{1}{\alpha}\left[(a w)\left(v \cdot b u_{1}\right)+(a v)\left(w b u_{1}\right)\right]=0 .
$$

Suponhamos, por hipótese de indução, que

$$
u_{s} \cdot w v=0, \quad \text { para } s=1, \ldots, i-1
$$


e verifiquemos a igualdade para $s=i$. Seja $u_{i} \in V_{\alpha, i}$, isto é $a b u_{i}=\alpha u_{i}+$ $x_{i-1}$, com $x_{i-1} \in V_{\alpha, i-1}$. Então por (3.3) e pela hipótese de indução, temos que

$$
\begin{aligned}
u_{i} \cdot w v & =\frac{1}{\alpha}\left[\left(a b u_{i}-x_{i-1}\right) \cdot w v\right]=\frac{1}{\alpha}\left[a b u_{i} \cdot w v-x_{i-1} \cdot w v\right] \\
& =\frac{1}{\alpha}\left[a b u_{i} \cdot w v\right]=-\frac{1}{\alpha}\left[a w \cdot v b u_{i}+a v \cdot w b u_{i}\right]=0
\end{aligned}
$$

Portanto $u_{i} \cdot w v=0$, para todo $i \geq 1$, o que mostra a igualdade.

O seguinte teorema nos mostra que não é possível construir (neste contexto) um contra-exemplo para a conjectura de Albert, quando a dimensão de $W_{0}$ é três.

Teorema 3.20 Não existem nilálgebras comutativas de potências associativas de nilíndice 4 , simples, com a seguinte forma

$$
\mathcal{B}=\mathcal{A} \oplus\langle w\rangle \oplus\left(\bigoplus_{0 \neq \alpha \in F} W_{\alpha}\right)
$$

sendo que $W_{0}=\mathcal{A} \oplus\langle w\rangle$ e algum $W_{\alpha} \neq 0$ com $\alpha \neq 0$.

Prova: Sabemos que $\mathcal{A} \cdot W_{\alpha} \subset W_{\alpha}, W_{\alpha}^{2} \subset W_{0,1}$ e $W_{\alpha} \cdot W_{\beta}=\{0\}$, para $\alpha \neq \beta \neq 0$ e pela observação anterior temos que neste caso $w \cdot \mathcal{A}=0$, logo nos resta calcular os produtos $w^{2}$ e $w \cdot W_{\alpha}$, para todo $\alpha \in F^{*}$. Dividiremos a prova em dois casos.

- Caso1: Existe $\alpha \neq 0$ tal que $W_{\alpha}^{2} \neq\{0\}$.

Sejam $u, v \in V_{\alpha}$ tal que $0 \neq u b b v \in W_{0,1}$. Então $u b b v=\theta a+\rho b+\lambda w$, com $\lambda \neq 0$, caso contrário $u b b v \in \mathcal{A}$ o que não é possível.

Uma vez que, $(u b b v)^{2}=-\frac{1}{2} u_{i}^{2}\left(b b v_{i}\right)^{2}=0$, por (3.2), temos que

$$
w^{2}=0
$$

Pelo Lema 3.6 temos, $0=u \cdot u b b v=\rho b u+\lambda w u$, logo

$$
w u=-\frac{\rho}{\lambda} b u \in W_{\alpha} .
$$


Da identidade $(3.2)$ segue, $b u \cdot u b b v=-\frac{1}{2} u^{2} \cdot b b b v=0 . \operatorname{Logo} 0=$ $b u \cdot u b b v=\theta a b u+\rho b b u+\lambda w b u$, portanto

$$
w b u=-\frac{\theta}{\lambda} a b u_{i}-\frac{\theta}{\lambda} b b u_{i} \in W_{\alpha} .
$$

E pela identidade (3.5)

$$
w b b u=-\frac{1}{2}\left[2 b b w u+2 b w b u+2 b u b w+2 u b b w+w u b^{2}+u w b^{2}\right]
$$

isto é

$$
w b b u_{i}=-b b w u_{i}-b w b u_{i} \in W_{\alpha} .
$$

Assim $w u, w b u, w b b u \in W_{\alpha}$.

Notemos que para qualquer $t \in V$, incluindo $t \in V_{\beta} \operatorname{com} \beta \in F^{*}$ e $\alpha \neq \beta$, temos que $t \cdot u b b v=0$, portanto

$$
w t=-\frac{\rho}{\lambda} b u_{t}, \quad \text { para todo } t \in V,
$$

e se existir $t, s \in V$, tal que tbbs $\neq 0$ e tbbs $=\theta_{t} a+\rho_{t} b+\lambda_{t} w$, então

$$
w t=-\frac{\rho_{t}}{\lambda_{t}} b t \text { para todo } t \in V
$$

portanto

$$
\frac{\rho}{\lambda}=\frac{\rho_{t}}{\lambda_{t}}
$$

para todo $t \in V$, como dado acima, se definimos

$$
\tilde{w}=\rho b+\lambda w,
$$

então é claro que $a \tilde{w}=b \tilde{w}=\tilde{w}^{2}=0$, e para qualquer $t \in V$,

$$
\begin{aligned}
\tilde{w} t & =\rho b t+\lambda w t=\rho b t-\lambda \frac{\rho}{\lambda} b t=0 \\
\tilde{w} b t & =\rho b b t+\lambda w b t=\rho b b t+\lambda\left(\frac{\theta_{t}}{\lambda_{t}} a b u_{t}-\frac{\rho_{t}}{\lambda_{t}} b b t\right) \\
& =\rho b b t-\lambda \frac{\theta_{t}}{\lambda_{t}} a b t-\lambda \frac{\rho}{\lambda} b b t=-\lambda \frac{\lambda_{t}}{\lambda_{t}} a b t, \\
\tilde{w} b b t & =-b b \tilde{w} t-b \tilde{w} b t=-\lambda \frac{\theta_{t}}{\lambda_{t}} a b b t,
\end{aligned}
$$


$\mathrm{e}$

$$
\begin{aligned}
t b b s & =\theta_{t} a+\rho_{t} b+\lambda_{t} w=\theta_{t} a+\lambda_{t} \frac{\rho}{\lambda} b+\lambda_{t} w \\
& =\theta_{t} a+\frac{\lambda_{t}}{\lambda}(\rho b+\lambda w)=\theta_{t} a+\frac{\lambda_{t}}{\lambda} \tilde{w} .
\end{aligned}
$$

Podemos concluir então, que para uma base adequada,

$$
w^{2}=w u=0, \quad w b b, w b u \in W_{\alpha} \quad \text { e } u b b v=\theta a+\lambda w
$$

para todo $u, v \in V_{\alpha}$, o que nos mostra que $b \notin \mathcal{B}^{2}$ e portanto

$$
\mathcal{B}^{2} \subsetneq \mathcal{B}
$$

- Caso 2: Para todo $\alpha \in F^{*}, W_{\alpha}^{2}=\{0\}$.

Seja $w u=\lambda_{1} a+\lambda_{2} b+\lambda_{3} w+\lambda_{4} x$, com $x \in \oplus_{0 \neq \alpha \in F} W_{\alpha}$ e $u \in V_{\alpha}$. Então por (3.17)

$$
0=u \cdot w u=\lambda_{2} b u+\lambda_{3} w u
$$

i) Se $\lambda_{3} \neq 0$, então

$$
w u=-\frac{\lambda_{2}}{\lambda_{3}} b u
$$

Analogamente ao caso anterior temos que $w b b u=-b b w u-b w b u$ e como $b b w u=0$, segue que

$$
w b b u=-b w b u
$$

Seja

$$
w b u=\gamma_{1} a+\gamma_{2} b+\gamma_{3} w+\gamma_{4} y,
$$

com $y \in \oplus_{0 \neq \alpha \in F} W_{\alpha}$. Então pela identidade (3.3) temos que $b u$. $w b u=-(b w)(u \cdot b u)-(b b u)(w u)=0, \log 0$

$$
0=\gamma_{1} a b u+\gamma_{2} b b u+\gamma_{3} w b u
$$


* Se $\gamma_{3} \neq 0$, então

$$
w b u=-\frac{\gamma_{1}}{\gamma_{3}} a b u-\frac{\gamma_{2}}{\gamma_{3}} b b u
$$

e temos então por (3.18), (3.19) e (3.20) que

$$
w u, w b u \text { e } w b b u \in W_{\alpha} .
$$

Seja $w^{2}=\theta_{1} a+\theta_{2} b+\theta_{3} w+\theta_{4} d, \quad \operatorname{com} d \in \oplus_{0 \neq \alpha \in F} W_{\alpha}$. Então $0=w^{2} w^{2}=\theta_{3}^{2} w^{2}+\tilde{d}$, com $\tilde{d} \in \oplus_{0 \neq \alpha \in F} W_{\alpha}$. Se $\theta_{3} \neq 0$, então $w^{2} \in \oplus_{0 \neq \alpha \in F} W_{\alpha}$, isto é

$$
w^{2}, w u, w b u \text { e } w b b u \in \oplus_{0 \neq \alpha \in F} W_{\alpha},
$$

o que mostra que $\mathcal{B}^{2} \subsetneq \mathcal{B}$. Se $\theta_{3}=0$, então $w^{2} \in \mathcal{B} \backslash\langle w\rangle$, isto é

$$
w^{2}, w u, w b u \text { e } w b b u \in \mathcal{B} \backslash\langle w\rangle,
$$

o que prova que $\mathcal{B}^{2} \subsetneq \mathcal{B}$.

$*$ Se $\gamma_{3}=0$, então $\gamma_{1}=\gamma_{2}=0, \operatorname{logo} w b u \in \oplus_{0 \neq \alpha \in F} W_{\alpha}$ e segue de (3.19) que $w b b u \in \oplus_{0 \neq \alpha \in F} W_{\alpha}$, portanto

$$
w u, w b u \text { e } w b b u \in \oplus_{0 \neq \alpha \in F} W_{\alpha}
$$

e analogamente ao caso anterior podemos concluir que

$$
w^{2}, w u, w b u \text { e } w b b u \in \oplus_{0 \neq \alpha \in F} W_{\alpha}
$$

e $\operatorname{assim} \mathcal{B}^{2} \subsetneq \mathcal{B}$.

ii) Se $\lambda_{3}=0$, então $\lambda_{2}=0$, de onde segue que $w u=\lambda_{1} a+\lambda_{4} v$. Seja

$$
w b u=\gamma_{1} a+\gamma_{2} b+\gamma_{3} w+\gamma_{4} v,
$$

com $v \in \oplus_{0 \neq \alpha \in F} W_{\alpha}$. Então pela identidade (3.3) temos que

$$
b u \cdot w b u=-(b w)(u \cdot b u)-(b b u)(w u)=\lambda_{1} a b b u,
$$

de onde segue que

$$
\gamma_{1} a b u+\gamma_{2} b b u+\gamma_{3} w b u-\lambda_{1} a b b u=0 .
$$

Portanto: 
* Se $\gamma_{3} \neq 0$, então $w b u_{i} \in W_{\alpha}$ e uma vez que $-w b b u=b b w u+$ bwbu, segue que $w b b u \in \oplus_{0 \neq \alpha \in F} W_{\alpha}$ e assim

$$
w u, w b u \text { e } w b b u \in\langle a\rangle \oplus\left(\bigoplus_{0 \neq \alpha \in F} W_{\alpha}\right) .
$$

Logo se $w^{2}=\theta_{1} a+\theta_{2} b+\theta_{3} w+\theta_{4} d, \quad \operatorname{com} d \in \oplus_{0 \neq \alpha \in F} W_{\alpha}$, então $0=w^{2} w^{2}=\theta_{3}^{2} w^{2}+\tilde{d}$, $\operatorname{com} \tilde{d} \in\langle a\rangle \oplus\left(\oplus_{0 \neq \alpha \in F} W_{\alpha}\right)$. Se $\theta_{3} \neq 0$, então $w^{2} \in\langle a\rangle \oplus\left(\oplus_{0 \neq \alpha \in F} W_{\alpha}\right)$, isto é

$$
w^{2}, w u, w b u \text { e } w b b u \in\langle a\rangle \oplus\left(\bigoplus_{0 \neq \alpha \in F} W_{\alpha}\right),
$$

o que mostra que $\mathcal{B}^{2} \subsetneq \mathcal{B}$. Se $\theta_{3}=0$, então $w^{2} \in \mathcal{B} \backslash\langle w\rangle$, isto é

$$
w^{2}, w u, w b u \text { e } w b b u \in \mathcal{B} \backslash\langle w\rangle,
$$

o que prova que $\mathcal{B}^{2} \subsetneq \mathcal{B}$.

* Se $\gamma_{3}=0$, então $\gamma_{1}=\gamma_{2}=\lambda_{1}=0$, portanto $w u, w b u \in$ $\oplus_{0 \neq \alpha \in F} W_{\alpha}$ e como $-w b b u=b b w u+b w b u$, segue que $w b b u \in$ $\oplus_{0 \neq \alpha \in F} W_{\alpha}$ e assim

$$
w u, w b u \text { e } w b b u \in \oplus_{0 \neq \alpha \in F} W_{\alpha},
$$

e da mesma forma como nos casos anteriores concluímos que $\mathcal{B}^{2} \subsetneq \mathcal{B}$.

Assim em qualquer dos casos vemos que

$$
\mathcal{B}^{2} \subsetneq \mathcal{B}
$$

Finalizamos esta seção com a seguinte conjectura.

Conjectura: Seja $\mathcal{B}$ uma nilálgebra comutativa de potências associativas de dimensão finita e nilíndice 4, sobre um corpo algebricamente fechado e de característica diferente de 2 e de 3 . Se existir uma decomposição $\mathcal{B}=$ $\bigoplus_{\alpha \in F} W_{\alpha}$ com algum $\alpha \neq 0$, então $\mathcal{B}$ não é simples. 
CAPÍTUlo 4

\section{Dimensão e nilíndice $n$.}

Neste capítulo daremos continuidade ao estudo das nilálgebras comutativas de potências associativas de dimensão e nilíndice $n$. Em [8], Correa e Suazo demonstraram a nilpotência de uma nilálgebra comutativa $\mathcal{B}$, de potências associativas de dimensão e nilíndice $n$, sobre um corpo de característica diferente de 2 e 3 . Neste trabalho classificaremos estas álgebras a menos de isomorfismo.

Estudaremos por separado os casos de dimensão 3,4,5,6 e 7. Para dimensão maior ou igual que 8, observamos um comportamento padrão em ditos produtos, o que nos levou a um resultado geral.

Para o restante do capítulo, $F$ denotará um corpo de característica diferente de 2,3 e 5 ou suficientemente grande comparada com $n$ e $\mathcal{B}$ uma nilálgebra sobre o corpo $F$. Se $x$ é um elemento de $\mathcal{B}$ tal que, $x^{k} \neq 0$ e $x^{k+1}=0$, para um inteiro positivo $k$, então $A_{x}$ denotará a subálgebra de $\mathcal{B}$, gerada por $\left\langle x, x^{2}, \ldots, x^{k}\right\rangle$.

Lema 4.1 Seja $\mathcal{B}$ uma nilálgebra comutativa de p.a. de dimensão n e nilíndice $n+1$. Se $x$ é um elemento em $\mathcal{B}$ tal que $x^{n} \neq 0$, então $\left\{x, x^{2}, \ldots, x^{n}\right\}$ é uma base de $\mathcal{B}$, e cada subálgebra própria de $\mathcal{B}$ está contida em $\mathcal{B}^{2}$.

Prova: Como $\mathcal{B}$ tem nilíndice $n+1$, existe $x \in \mathcal{B}$ tal que $x^{n} \neq 0$ e já que $\left\{x, x^{2}, \ldots, x^{n}\right\}$ é um conjunto linearmente independente, é claro que é uma base de $\mathcal{B}$. Seja $D$ uma subálgebra de $\mathcal{B}$ tal que $D \nsubseteq \mathcal{B}^{2}$. Logo, existe um 
elemento $d \in D$ tal que $d \notin \mathcal{B}^{2}$. O elemento $d$ exprime-se em função da base como $d=\alpha_{1} x+\ldots+\alpha_{n} x^{n}$ com $\alpha_{1} \neq 0$. Então $d^{s}=\alpha_{1}^{s} x^{s}+f_{s}(x) x^{s+1}$ para $s \geq 1$, onde $f_{s}(x)$ é uma expressão polinomial em $x$. Logo, $d^{n}=\alpha_{1}^{n} x^{n} \neq 0$ e então o conjunto $\left\{d, d^{2}, \ldots, d^{n}\right\}$ é linearmente independente, $\operatorname{logo} D$ tem dimensão $n$ e portanto $D=\mathcal{B}$.

Teorema 4.2 [8] Seja $\mathcal{B}$ uma nilálgebra comutativa de p.a. de dimensão e nilíndice $n$, sobre um corpo de característica diferente de 2 e de 3. Se a é um elemento em $\mathcal{B}$ tal que $a^{n-1} \neq 0$, então $\mathcal{B}^{k}=A_{a}^{k}$ para todo $k \geq 2$. Portanto $\mathcal{B}$ é nilpotente de indice $n$.

Observamos que para cada $x \in \mathcal{B}$ o elemento $x^{n-1}$ está no anulador da álgebra $\mathcal{B}$.

Corolário 4.3 Seja $\mathcal{B}$ uma nilálgebra comutativa de p.a. de dimensão e nilíndice $n$, sobre um corpo de característica diferente de 2 e de 3. Se a é um elemento em $\mathcal{B}$ tal que $a^{n-1} \neq 0$, então existe $b \in \mathcal{B} \backslash A_{a}$ tal que ba $=0$. $O$ elemento b está determinado de maneira única, a menos de multiplicação por escalares diferentes de zero e somas de elementos em $\left\langle a^{n-1}\right\rangle$, isto é, se $b_{1}$ é outro elemento com a mesma propriedade, então existe $\lambda, \mu \in F, \lambda \neq 0$ tal que $b_{1}=\lambda b+\mu a^{n-1}$.

Prova: Seja $c \in \mathcal{B} \backslash A_{a}$. Então $\left\{c, a, a^{2}, \ldots, a^{n-1}\right\}$ é uma base de $\mathcal{B}$ e $c a$ é da forma $c a=\alpha_{2} a^{2}+\cdots+\alpha_{n-1} a^{n-1}$, onde $\alpha_{2}, \ldots, \alpha_{n-1} \in F$. Seja $b=\lambda_{0} c+\lambda_{1} a+\lambda_{2} a^{2}+\cdots+\lambda_{n-1} a^{n-1}$ um elemento arbitrário em $\mathcal{B}$. Então $b \in \mathcal{B} \backslash A_{a}$ e $b a=0$ se e somente se $\lambda_{0} \neq 0$ e $0=b a=\lambda_{0} c a+\sum_{i=1}^{n-2} \lambda_{i} a^{i+1}$. Portanto, $\lambda_{0} \neq 0$ e $c a=-\left(\sum_{i=1}^{n-2} \frac{\lambda_{i}}{\lambda_{0}} a^{i+1}\right)$.

Denotaremos por $\mathcal{P}(\mathcal{B})$, o conjunto formado pelos seguintes pares de elementos,

$$
\mathcal{P}(\mathcal{B})=\left\{(a, b) \in \mathcal{B} \times \mathcal{B} \quad \mid \quad a^{n-1} \neq 0, \quad a b=0 \quad \text { e } \quad b \notin A_{a}\right\} .
$$


Fazendo $x=a$ e $y=b$ em (1.2), (1.1) e (1.4), e utilizando o fato de $a b=0$ obtemos as seguintes identidades

$$
\begin{aligned}
a^{2} b^{2} & =\frac{1}{2}\left[a\left(a b^{2}\right)+b\left(a^{2} b\right)\right], \\
a^{3} b & =-a\left(a^{2} b\right), \\
a^{4} b & =\frac{1}{3}\left[3 a^{2}\left(a^{2} b\right)-2 a\left(a^{3} b\right)-2 a\left(a\left(a^{2} b\right)\right)\right], \\
a^{5} b & =\frac{1}{3}\left[4 a^{2}\left(a^{3} b\right)-a^{3}\left(a^{2} b\right)-2 a\left(a^{4} b\right)-2 a\left(a\left(a^{3} b\right)\right)\right], \\
a^{r+2} b & =\frac{1}{3}\left[4 a^{2}\left(a^{r} b\right)-a^{r}\left(a^{2} b\right)-2 a\left(a^{r+1} b\right)-2 a\left(a\left(a^{r} b\right)\right)\right] .
\end{aligned}
$$

\subsection{Dimensão menor ou igual a quatro.}

Para dimensão menor ou igual a 4, Gerstenhaber e Myung [12] mostraram a nilpotência destas álgebras determinando suas classes de isomorfismos.

Para dimensão 3, os autores do trabalho citado acima, acharam uma família de álgebras associativas. Para cada $\alpha \in F, \mathcal{B}(\alpha)$ é uma nilálgebra comutativa com base $\left\{a, a^{2}, b\right\}$, onde $b^{2}=\alpha a^{2}$, e os outros produtos são nulos, isto é, $a^{2}$ está no anulador da álgebra. Para cada $\alpha, \alpha^{\prime} \in F$, cumpre-se que $\mathcal{B}(\alpha) \cong \mathcal{B}\left(\alpha^{\prime}\right)$ se, e somente se, existir $\rho \in F^{*}$ tal que $\alpha^{\prime}=\rho^{2} \alpha$.

Para dimensão 4, Gerstenhaber e Myung acharam a família de álgebras, definidas a seguir. Para cada $\alpha \in F$, a nilálgebra comutativa $\mathcal{C}(\alpha)$ tem uma base da forma $\left\{a, a^{2}, a^{3}, b\right\}$, tal que $b A_{a}^{2}=0$ e

$$
a b=0 \quad \text { e } \quad b^{2}=\alpha a^{2}
$$

Esta familia de álgebras está parametrizada por elementos de $F^{*} /\left(F^{*}\right)^{2}$. Também cinco álgebras individuais com base $\left\{a, a^{2}, a^{3}, b\right\}$ tal que $b A_{a}^{2}=0$ e os produtos $a b$ e $b^{2}$ são dados por um da seguinte lista: 


$$
\begin{aligned}
& \mathcal{C}_{1}: \quad a b=0, \quad b^{2}=0 ; \\
& \mathcal{C}_{2}: \quad a b=0, \quad b^{2}=a^{3} ; \\
& \mathcal{C}_{3}: \quad a b=a^{2}, \quad b^{2}=0 ; \\
& \mathcal{C}_{4}: \quad a b=a^{2}, \quad b^{2}=a^{3} ; \\
& \mathcal{C}_{5}: \quad a b=a^{2}, \quad b^{2}=a^{2} .
\end{aligned}
$$

Observamos que somente são associativas as álgebras determinadas por $\mathcal{C}_{1}$ e $\mathcal{C}_{2}$. Este resultado pode ser reformulado, usando diferentes bases, no seguinte teorema.

Teorema 4.4 Uma nilálgebra comutativa de p.a. de dimensão e nilíndice 4, é isomorfa a uma e somente uma álgebra com base $\left\{b, a, a^{2}, a^{3}\right\}$, e produtos não triviais e não zero conforme um dos casos que aparecem na seguinte lista:

$$
\begin{array}{lll}
\mathcal{A}_{1}: & b^{2}=-a^{2}+a^{3}, & a^{2} b=a^{3} . \\
\mathcal{A}_{2}: & b^{2}=-a^{2}, & a^{2} b=a^{3} ; \\
\mathcal{A}_{3}: & & a^{2} b=a^{3} ;
\end{array}
$$

$\mathcal{A}_{4}: \quad b^{2}=a^{3}$

$$
\mathcal{A}_{5}(\alpha): \quad b^{2}=\alpha a^{2}, \quad\left[\rho^{2}\right] ;
$$

A matriz da direita dá a condição necessária e suficiente para que duas álgebras na classe sejam isomorfas, isto é $\mathcal{A}_{5}(\alpha) \cong \mathcal{A}_{5}\left(\alpha^{\prime}\right)$ se e somente se, existir $\rho \in F^{*}$ tal que $\alpha^{\prime}=\rho^{2} \alpha$. Por produtos não triviais, entendemos os produtos entre as potências de $a$.

\subsection{Classificação para dimensão cinco.}

Nesta seção estudaremos as nilálgebras comutativas de p.a. de dimensão e nilíndice 5. Daremos a tabela de multiplicação para uma base da forma 
$\left\{b, a, a^{2}, a^{3}, a^{4}\right\}$, com $a b=0$, e também uma classificação a menos de isomorfismos.

O seguinte teorema foi provado por Elgueta e Suazo em [10] e Correa e Suazo em [8].

Teorema 4.5 Seja $\mathcal{B}$ é uma nilálgebra comutativa, de p.a. de dimensão e nilíndice 5 , e $(a, b) \in \mathcal{P}(\mathcal{B})$. Então:

(i) Se $\mathcal{B}$ não é de Jordan, então existe $(a, b) \in \mathcal{P}(\mathcal{B})$, tal que

$$
b^{2}+a^{2} \in A_{a}^{3}, b a^{2}-a^{3} \in A_{a}^{4}, b a^{3}+a^{4}=0 .
$$

(ii) $S e \mathcal{B}$ é de Jordan, então existe uma base da forma $\left\{b, a, a^{2}, a^{3}, a^{4}\right\}$ tal que

$$
b^{2} \in A_{a}^{3}, b a \in A_{a}^{3} .
$$

Vejamos agora o seguinte resultado.

Teorema 4.6 Seja $\mathcal{B}$ uma nilálgebra comutativa de p.a. de dimensão e nilíndice 5 e seja $(a, b) \in \mathcal{P}(\mathcal{B})$. Então para a base $\left\{b, a, a^{2}, a^{3}, a^{4}\right\}$ de $\mathcal{B}$, os produtos não triviais são dados por:

$$
\begin{aligned}
b^{2} & =-\gamma_{3}^{2} a^{2}+\alpha_{3} a^{3}+\alpha_{4} a^{4}, \\
a^{2} b & =\gamma_{3} a^{3}+\gamma_{4} a^{4}, \\
a^{3} b & =-\gamma_{3} a^{4} .
\end{aligned}
$$

Reciprocamente, se $\mathcal{B}$ é uma nilálgebra comutativa com base $\left\{b, a, a^{2}, a^{3}, a^{4}\right\}$ e produtos $a b=a^{k}=0, a^{i} a^{j}=a^{i+j}$ para todo $k \geq 5$ e para todos os inteiros positivos $i$ e $j$, e (4.6), então $\mathcal{B}$ é de p.a. e nilíndice 5 .

Prova: Seja $\mathcal{B}$ uma nilálgebra comutativa de p.a. de dimensão e nilíndice 5 e $(a, b) \in \mathcal{P}(\mathcal{B})$. Por Teorema 4.2, temos que

$$
b^{2}=\alpha_{2} a^{2}+\alpha_{3} a^{3}+\alpha_{4} a^{4} \quad \text { e } \quad a^{2} b=\gamma_{3} a^{3}+\gamma_{4} a^{4} .
$$

Usando relações (4.2) e (4.1), obtemos que

$$
a^{3} b=-a\left(\gamma_{3} a^{3}+\gamma_{4} a^{4}\right)=-\gamma_{3} a^{4}
$$


e

$$
a^{2} b^{2}=\frac{1}{2}\left[\alpha_{2} a^{4}+\gamma_{3} a^{3} b+\gamma_{4} a^{4} b\right]=\frac{1}{2}\left[\alpha_{2} a^{4}-\gamma_{3}^{2} a^{4}\right] .
$$

Por outro lado, temos que

$$
a^{2} b^{2}=\alpha_{2} a^{4}
$$

$\operatorname{logo} \alpha_{2}=-\gamma_{3}^{2}$. Assim na base anterior, os produtos não triviais, são dados por

$$
b^{2}=-\gamma_{3}^{2} a^{2}+\alpha_{3} a^{3}+\alpha_{4} a^{4}, \quad a^{2} b=\gamma_{3} a^{3}+\gamma_{4} a^{4} \quad \text { e } \quad a^{3} b=-\gamma_{3} a^{4} .
$$

Demonstraremos agora que, se $\mathcal{B}$ tem base $\left\{b, a, a^{2}, a^{3}, a^{4}\right\}$, com produtos dados por $a b=a^{k}=0, a^{i} a^{j}=a^{i+j}$, para todo $k \geq 5$ e $i, j \geq 1$, e relações (4.6), então $\mathcal{B}$ é de p.a. e nilíndice 5. Para tal seja $x=\lambda b+\sum_{i=1}^{4} \lambda_{i} a^{i}$ um elemento arbitrário da álgebra. Então

$$
\begin{aligned}
x^{2}= & \left(-\lambda^{2} \gamma_{3}^{2}+\lambda_{1}^{2}\right) a^{2}+\left(\lambda^{2} \alpha_{3}+2 \lambda \lambda_{2} \gamma_{3}+2 \lambda_{1} \lambda_{2}\right) a^{3}+\left(\lambda^{2} \alpha_{4}+2 \lambda \lambda_{2} \gamma_{4}\right. \\
& \left.-2 \lambda \lambda_{3} \gamma_{3}+2 \lambda_{1} \lambda_{3}+\lambda_{2}^{2}\right) a^{4}, \\
x^{3}= & \left(-\lambda^{3} \gamma_{3}^{3}+\lambda \lambda_{1}^{2} \gamma_{3}-\lambda_{1} \lambda^{2} \gamma_{3}^{2}+\lambda_{1}^{3}\right) a^{3}+\left(\lambda^{3} \gamma_{3}^{2} \gamma_{4}+\lambda \lambda_{1}^{2} \gamma_{4}-\lambda^{3} \gamma_{3} \alpha_{3}\right. \\
& \left.-3 \lambda^{2} \lambda_{2} \gamma_{3}^{2}+\lambda_{1} \lambda^{2} \alpha_{3}+3 \lambda_{1}^{2} \lambda_{2}\right) a^{4}, \\
x^{4}= & \left(\lambda^{4} \gamma_{3}^{4}-2 \lambda^{2} \lambda_{1}^{2} \gamma_{3}^{2}+\lambda_{1}^{4}\right) a^{4}=\left(\lambda^{2} \gamma_{3}^{2}-\lambda_{1}^{2}\right)^{2} a^{4}, \\
x^{5}= & 0, \\
x^{2} x^{2}= & \left(\lambda^{2} \gamma_{3}^{2}-\lambda_{1}^{2}\right)^{2} a^{4},
\end{aligned}
$$

portanto $x^{4}-x^{2} x^{2}=0$, ficando claro que $\mathcal{B}$ é de p.a. e nilíndice 5 .

Denotaremos a álgebra do enunciado do lema anterior por $\mathcal{B}\left(\alpha_{3}, \alpha_{4}, \gamma_{3}, \gamma_{4}\right)$. Observamos que a matriz coordenada por linhas de $L_{b}$, em relação à base $\left\{b, a, a^{2}, a^{3}, a^{4}\right\}$ é

$$
\left(\begin{array}{ccccc}
0 & 0 & -\gamma_{3}^{2} & \alpha_{3} & \alpha_{4} \\
0 & 0 & 0 & 0 & 0 \\
0 & 0 & 0 & \gamma_{3} & \gamma_{4} \\
0 & 0 & 0 & 0 & -\gamma_{3} \\
0 & 0 & 0 & 0 & 0
\end{array}\right) .
$$


A seguir, faremos uma análise que nos permitirá determinar as classes de isomorfismo das álgebras da forma $\mathcal{B}\left(\alpha_{3}, \alpha_{4}, \gamma_{3}, \gamma_{4}\right)$.

Lema 4.7 Sejam $\lambda$ e $\beta$ escalares não nulos. Então

$$
\mathcal{B}\left(\alpha_{3}, \alpha_{4}, \gamma_{3}, \gamma_{4}\right) \cong \mathcal{B}\left(\frac{\beta^{2}}{\lambda^{3}} \alpha_{3}, \frac{\beta^{2}}{\lambda^{4}} \alpha_{4}, \frac{\beta}{\lambda} \gamma_{3}, \frac{\beta}{\lambda^{2}} \gamma_{4}\right) .
$$

Prova: Seja $\mathcal{B}$ a álgebra $\mathcal{B}\left(\alpha_{3}, \alpha_{4}, \gamma_{3}, \gamma_{4}\right)$, em relação à base $\left\{b, a, a^{2}, a^{3}, a^{4}\right\}$. Considerar $\lambda$ e $\beta$ em $F^{*}$ e os elementos em $\mathcal{B}$ dados por $a_{1}:=\lambda a$ e $b_{1}:=\beta b$. Obviamente $\left(a_{1}, b_{1}\right) \in \mathcal{P}(\mathcal{B})$. Determinemos agora as constantes de estrutura de $\mathcal{B}$ em relação à base de $\mathcal{B}$ determinada pelo par $\left(a_{1}, b_{1}\right)$. Temos que $a_{1}^{2}=\lambda^{2} a^{2}, a_{1}^{3}=\lambda^{3} a^{3}$ e $a_{1}^{4}=\lambda^{4} a^{4}, \log \mathrm{O}$

$$
b_{1}^{2}=\beta^{2} b^{2}=-\beta^{2} \gamma_{3}^{2} \frac{1}{\lambda^{2}} a_{1}^{2}+\beta^{2} \alpha_{3} \frac{1}{\lambda^{3}} a_{1}^{3}+\beta^{2} \alpha_{4} \frac{1}{\lambda^{4}} a_{1}^{4},
$$

e também

$$
a_{1}^{2} b_{1}=\lambda^{2} \beta a^{2} b=\frac{\beta}{\lambda} \gamma_{3} a_{1}^{3}+\frac{\beta}{\lambda^{2}} \gamma_{4} a_{1}^{4}
$$

o que demonstra o lema.

Lema 4.8 Para todo $\rho$ em F,

$$
\mathcal{B}\left(\alpha_{3}, \alpha_{4}, \gamma_{3}, \gamma_{4}\right) \cong \mathcal{B}\left(\alpha_{3}, \alpha_{4}-3 \rho \alpha_{3}, \gamma_{3}, \gamma_{4}-6 \rho \gamma_{3}\right) .
$$

Prova: Seja $\mathcal{B}$ a álgebra $\mathcal{B}\left(\alpha_{3}, \alpha_{4}, \gamma_{3}, \gamma_{4}\right)$ em relação à base determinada pelo par $(a, b)$ em $\mathcal{P}(\mathcal{B})$. Seja $\rho \in F$. Definamos os elementos em $\mathcal{B}$,

$$
a_{1}:=a+\rho a^{2} \text { e } b_{1}:=b-\rho \gamma_{3} a^{2}+\rho\left(\rho \gamma_{3}-\gamma_{4}\right) a^{3} .
$$

Então $a_{1}^{2}=a^{2}+2 \rho a^{3}+\rho^{2} a^{4}, a_{1}^{3}=a^{3}+3 \rho a^{4}, a_{1}^{4}=a^{4} \mathrm{e}$

$$
\begin{aligned}
a_{1} b_{1} & =-\rho \gamma_{3} a^{3}+\rho\left(\rho \gamma_{3}-\gamma_{4}\right) a^{4}+\rho a^{2} b-\rho^{2} \gamma_{3} a^{4} \\
& =-\rho \gamma_{3} a^{3}+\rho\left(\rho \gamma_{3}-\gamma_{4}\right) a^{4}+\rho \gamma_{3} a^{3}+\rho \gamma_{4} a^{4}-\rho^{2} \gamma_{3} a^{4} \\
& =\left(\rho^{2} \gamma_{3}-\rho \gamma_{4}+\rho \gamma_{4}-\rho^{2} \gamma_{3}\right) a^{4} \\
& =0
\end{aligned}
$$


Portanto, provamos que $\left(a_{1}, b_{1}\right) \in \mathcal{P}(\mathcal{B})$. Determinemos agora as constantes de estrutura de $\mathcal{B}$ em relação à base $\left\{b_{1}, a_{1}, a_{1}^{2}, a_{1}^{3}, a_{1}^{4}\right\}$. Temos que

$$
\begin{aligned}
b_{1}^{2} & =b^{2}-2 \rho \gamma_{3} a^{2} b+2 \rho\left(\rho \gamma_{3}-\gamma_{4}\right) a^{3} b+\rho^{2} \gamma_{3}^{2} a^{4} \\
& =-\gamma_{3}^{2} a^{2}+\alpha_{3} a^{3}+\alpha_{4} a^{4}-2 \rho \gamma_{3}^{2} a^{3}-2 \rho \gamma_{3} \gamma_{4} a^{4}-2 \rho \gamma_{3}\left(\rho \gamma_{3}-\gamma_{4}\right) a^{4}+\rho^{2} \gamma_{3}^{2} a^{4} \\
& =-\gamma_{3}^{2} a^{2}+\left(\alpha_{3}-2 \rho \gamma_{3}^{2}\right) a^{3}+\left(\alpha_{4}-\rho^{2} \gamma_{3}^{2}\right) a^{4},
\end{aligned}
$$

e

$$
\begin{aligned}
a_{1}^{2} b_{1} & =b a^{2}+2 \rho a^{3} b-\rho \gamma_{3} a^{4}=\gamma_{3} a^{3}+\gamma_{4} a^{4}-2 \rho \gamma_{3} a^{4}-\rho \gamma_{3} a^{4} \\
& =\gamma_{3} a^{3}+\left(\gamma_{4}-3 \rho \gamma_{3}\right) a^{4} .
\end{aligned}
$$

Usando agora as relações $a^{3}=a_{1}^{3}-3 \rho a_{1}^{4}$ e $a^{2}=a_{1}^{2}-2 \rho\left(a_{1}^{3}-3 \rho a_{1}^{4}\right)-\rho^{2} a_{1}^{4}=$ $a_{1}^{2}-2 \rho a_{1}^{3}+5 \rho^{2} a_{1}^{4}$, segue que

$$
\begin{aligned}
b_{1}^{2}= & -\gamma_{3}^{2}\left(a_{1}^{2}-2 \rho a_{1}^{3}+5 \rho^{2} a_{1}^{4}\right)+\left(\alpha_{3}-2 \rho \gamma_{3}^{2}\right)\left(a_{1}^{3}-3 \rho a_{1}^{4}\right)+\left(\alpha_{4}-\rho^{2} \gamma_{3}^{2}\right) a_{1}^{4} \\
= & -\gamma_{3}^{2} a_{1}^{2}+2 \gamma_{3}^{2} \rho a_{1}^{3}-5 \rho^{2} \gamma_{3}^{2} a_{1}^{4}+\left(\alpha_{3}-2 \rho \gamma_{3}^{2}\right) a_{1}^{3}-3 \rho\left(\alpha_{3}-2 \rho \gamma_{3}^{2}\right) a_{1}^{4} \\
& +\left(\alpha_{4}-\rho^{2} \gamma_{3}^{2}\right) a_{1}^{4}=-\gamma_{3}^{2} a_{1}^{2}+\alpha_{3} a_{1}^{3}+\left(\alpha_{4}-3 \rho \alpha_{3}\right) a_{1}^{4},
\end{aligned}
$$

e

$$
a_{1}^{2} b_{1}=\gamma_{3}\left(a_{1}^{3}-3 \rho a_{1}^{4}\right)+\left(\gamma_{4}-3 \rho \gamma_{3}\right) a_{1}^{4}=\gamma_{3} a_{1}^{3}+\left(\gamma_{4}-6 \rho \gamma_{3}\right) a_{1}^{4}
$$

demonstrando o lema.

Observamos que pelo Lema 4.7, se $\gamma_{3} \neq 0$, então tomando $\beta=\frac{1}{\gamma_{3}}$ e $\lambda=1$, obtemos que

$$
\mathcal{B}\left(\alpha_{3}, \alpha_{4}, \gamma_{3}, \gamma_{4}\right) \cong \mathcal{B}\left(\frac{\alpha_{3}}{\gamma_{3}^{2}}, \frac{\alpha_{4}}{\gamma_{3}^{2}}, 1, \frac{\gamma_{4}}{\gamma_{3}}\right)
$$

Portanto podemos supor, a menos de isomorfismos, que $\gamma_{3}=0$ ou $\gamma_{3}=1$. Analisemos estes dois casos separadamente.

- Caso 1: $\left(\gamma_{3}=0\right.$.) Temos a álgebra $\mathcal{B}\left(\alpha_{3}, \alpha_{4}, 0, \gamma_{4}\right)$, que pelo Lema 4.8, é isomorfa à álgebra $\mathcal{B}\left(\alpha_{3}, \alpha_{4}-3 \rho \alpha_{3}, 0, \gamma_{4}\right)$, para todo $\rho$ em $F$. 
- Se $\alpha_{3} \neq 0$, então fazendo $\rho=\frac{\alpha_{4}}{3 \alpha_{3}}$, temos que $\mathcal{B}\left(\alpha_{3}, \alpha_{4}, 0, \gamma_{4}\right)$, é isomorfa à álgebra $\mathcal{B}\left(\alpha_{3}, 0,0, \gamma_{4}\right)$. Por outro lado, temos do Lema 4.7, que se $\beta=\lambda=\alpha_{3} \neq 0$, então $\mathcal{B}\left(\alpha_{3}, 0,0, \gamma_{4}\right)$ é isomorfa à álgebra $\mathcal{B}\left(1,0,0, \gamma_{4}^{\prime}\right)$, onde $\gamma_{4}^{\prime}=\gamma_{4} / \alpha_{3}$.

* Se $\gamma_{4}^{\prime} \neq 0$, então considerando $\lambda=\gamma_{4}^{\prime 2}$ e $\beta=\gamma_{4}^{\prime 3}$, no Lema 4.7 , obtemos que

$$
\mathcal{B}\left(1,0,0, \gamma_{4}^{\prime}\right) \cong \mathcal{B}(1,0,0,1)
$$

$*$ Se $\gamma_{4}^{\prime}=0$, então

$$
\mathcal{B}\left(1,0,0, \gamma_{4}^{\prime}\right) \cong \mathcal{B}(1,0,0,0)
$$

- Se $\alpha_{3}=0$, temos a álgebra $\mathcal{B}\left(0, \alpha_{4}, 0, \gamma_{4}\right)$.

* Se $\gamma_{4} \neq 0$, então tomando $\beta=\lambda=\gamma_{4}$, no Lema 4.7, obtemos que

$$
\mathcal{B}\left(0, \alpha_{4}, 0, \gamma_{4}\right) \cong \mathcal{B}\left(0, \alpha_{4}^{\prime}, 0,1\right),
$$

onde $\alpha_{4}^{\prime}=\alpha_{4} / \gamma_{4}^{2}$.

* Se $\gamma_{4}=0$, temos a álgebra $\mathcal{B}\left(0, \alpha_{4}, 0,0\right)$.

- Caso 2: $\left(\gamma_{3}=1\right.$.) Então temos a álgebra $\mathcal{B}\left(\alpha_{3}, \alpha_{4}, 1, \gamma_{4}\right)$, que pelo Lema 4.8, é isomorfa à álgebra $\mathcal{B}\left(\alpha_{3}, \alpha_{4}-3 \rho \alpha_{3}, 1, \gamma_{4}-6 \rho\right)$, para todo $\rho$ em $F$. Logo, podemos assumir a menos de isomorfismo, que $\gamma_{4}=0$.

- Se $\alpha_{3}=0$, temos a álgebra $\mathcal{B}\left(0, \alpha_{4}, 1,0\right)$.

- Se $\alpha_{3} \neq 0$, então fazendo $\beta=\lambda=\alpha_{3} \neq 0$, no Lema 4.7, obtemos que a álgebra $\mathcal{B}\left(\alpha_{3}, \alpha_{4}, 1,0\right)$, é isomorfa à álgebra $\mathcal{B}\left(1, \alpha_{4}^{\prime}, 1,0\right)$, onde $\alpha_{4}^{\prime}=\frac{\alpha_{4}}{\alpha_{3}^{2}}$.

Podemos concluir, de todo o anterior que, se $\mathcal{B}$ é uma nilálgebra comutativa de p.a. de dimensão e nilíndice 5 , então existe uma base da forma $\left\{b, a, a^{2}, a^{3}, a^{4}\right\}$, cujos produtos são dados por uma da seguinte lista: 


$$
\begin{aligned}
& \mathcal{B}_{1}(\alpha): \quad b^{2}=-a^{2}+a^{3}+\alpha a^{4}, \quad a^{2} b=a^{3}, \quad a^{3} b=-a^{4}, \quad \alpha \in F ; \\
& \mathcal{B}_{2}(\alpha): \quad b^{2}=-a^{2}+\alpha a^{4}, \quad a^{2} b=a^{3}, \quad a^{3} b=-a^{4}, \quad \alpha \in F ; \\
& \mathcal{B}_{3}(\alpha): \quad b^{2}=\alpha a^{4}, \quad \quad a^{2} b=a^{4}, \quad \alpha \in F ; \\
& \mathcal{B}_{4}(\alpha): \quad b^{2}=\alpha a^{4}, \quad \alpha \neq 0 ; \\
& \mathcal{B}_{5}: \quad b^{2}=a^{3}, \quad a^{2} b=a^{4} ; \\
& \mathcal{B}_{6}: \quad b^{2}=a^{3}
\end{aligned}
$$

e os outros produtos não triviais são zero. Portanto toda álgebra desta classe é isomorfa a alguma da lista anterior. Analisemos agora as condições de isomorfismo entre as álgebras da lista dada acima.

Observamos que $\operatorname{Dim}\left(\operatorname{Anul}\left(\mathcal{B}_{7}\right)\right)=\operatorname{Dim}\left(\left\langle b, a^{4}\right\rangle\right)=2$ e $\operatorname{Dim}\left(\operatorname{Anul}\left(\mathcal{B}_{i}\right)\right)=$ $\operatorname{Dim}\left(\left\langle a^{4}\right\rangle\right)=1$, para $i \neq 7$, e portanto podemos concluir que,

$$
\mathcal{B}_{7} \not \mathcal{B}_{i}, \quad \text { para } i \neq 7 \text {. }
$$

Pelo Teorema 4.5, temos que as álgebras $\mathcal{B}_{1}(\alpha)$ e $\mathcal{B}_{2}(\alpha)$ não são de Jordan e que $\mathcal{B}_{3}(\alpha), \mathcal{B}_{4}(\alpha), \mathcal{B}_{5}$ e $\mathcal{B}_{6}$, são álgebras de Jordan. Portanto podemos separar as seis primeiras álgebras da lista anterior, em dois conjuntos de álgebras não isomorfas, isto é, uma álgebra de um conjunto não é isomorfa a nenhuma outra álgebra do outro conjunto.

Seja $\overline{\mathcal{B}}_{i}=\mathcal{B}_{i} /\left(\operatorname{Anul}\left(\mathcal{B}_{i}\right)\right)$. Então para $i=5,6$, a dimensão do anulador de $\overline{\mathcal{B}}_{i}$ é 1 e para $j=3,4$, a dimensão do anulador de $\overline{\mathcal{B}}_{j}$ é 2 . Portanto o segundo conjunto fica separado em dois subconjuntos de álgebras não isomorfas. Lembrando que a álgebra $\mathcal{B}_{7}$ não é isomorfa a nenhuma da lista anterior, temos as restantes álgebras desta lista separadas em três conjuntos de álgebras não isomorfas, a saber:

$$
C 1: \mathcal{B}_{1}(\alpha), \mathcal{B}_{2}(\alpha), \quad C 2: \mathcal{B}_{3}(\alpha), \mathcal{B}_{4}(\alpha), \quad C 3: \mathcal{B}_{5}, \mathcal{B}_{6}
$$

Analisemos então cada conjunto por separado. 
Observamos no conjunto $C 1$ que a álgebra $\overline{\mathcal{B}}_{2}(\alpha)$ corresponde a álgebra $\mathcal{A}_{3}$ e a álgebra $\overline{\mathcal{B}}_{1}(\alpha)$ corresponde a álgebra $\mathcal{A}_{1}$, do Teorema 4.4 , e já que estas álgebras não são isomorfas, segue que para todo $\alpha$ e $\alpha^{\prime}$ em $F$

$$
\mathcal{B}_{1}(\alpha) \neq \mathcal{B}_{2}\left(\alpha^{\prime}\right)
$$

Podemos concluir de todo o anterior que

$$
\mathcal{B}_{1} ¥ \mathcal{B}_{i}, \quad \text { para } i \neq 1 \quad \text { e } \quad \mathcal{B}_{2} ¥ \mathcal{B}_{j} \text {, para } j \neq 2 \text {. }
$$

As álgebras do conjunto $C 3$, são isomorfas como demonstramos no seguinte lema.

Lema 4.9 Temos que

$$
\mathcal{B}_{5} \cong \mathcal{B}_{6}
$$

Prova: Seja $\left\{b, a, a^{2}, a^{3}, a^{4}\right\}$ a base $\mathcal{B}_{6}$, onde $b^{2}=a^{3}$ e os outros produtos não triviais são zero. Se definimos

$$
a_{1}:=-b+a \quad \text { e } \quad b_{1}:=b+a^{2},
$$

então $a_{1} b_{1}=-b^{2}-a^{2} b+a b+a^{3}=-b^{2}+a^{3}=0, a_{1}^{2}=b^{2}-2 a b+a^{2}=a^{2}+a^{3}$, $a_{1}^{3}=a^{3}+a^{4}$ e $a_{1}^{4}=a^{4} \neq 0$. É claro da definição de $b_{1}$ que $b_{1} \notin A_{a_{1}}$, portanto podemos concluir, de todo o anterior, que $\left(a_{1}, b_{1}\right) \in \mathcal{P}\left(\mathcal{B}_{6}\right)$. Como

$$
\begin{aligned}
b_{1}^{2} & =b^{2}+2 a^{2} b+a^{4}=a^{3}+a^{4}=a_{1}^{3}, \\
a_{1}^{2} b_{1} & =a^{4}=a_{1}^{4}, \\
a_{1}^{3} b_{1} & =0,
\end{aligned}
$$

temos que $\mathcal{B}_{5}$ e $\mathcal{B}_{6}$ são isomorfas.

Observamos que

$$
\mathcal{B}_{6} \not \mathcal{B}_{i}, \quad \text { para } i \neq 5,6 \text {. }
$$

Vejamos agora que para cada $\alpha$ e $\alpha^{\prime}$ em $F$, a álgebra $\mathcal{B}_{3}(\alpha)$ não é isomorfa à álgebra $\mathcal{B}_{4}\left(\alpha^{\prime}\right)$, o que será uma consequência do seguinte lema. 
Lema 4.10 Seja $\alpha$ em $F^{*}$. Se $\left(a_{1}, b_{1}\right)$ está em $\mathcal{P}\left(\mathcal{B}_{4}(\alpha)\right)$, então existe $\rho \in F^{*}$ tal que

$$
b_{1}^{2}=\rho^{2} \alpha a_{1}^{4} \quad e \quad a_{1}^{2} b_{1}=0,
$$

e os outros produtos não triviais são zero.

Prova: Seja $\left\{b, a, a^{2}, a^{3}, a^{4}\right\}$ uma base para $\mathcal{B}_{4}(\alpha)$, tal que $b^{2}=\alpha a^{4}$ e $a^{2} b=$ 0 . Se $\left(a_{1}, b_{1}\right) \in \mathcal{P}\left(\mathcal{B}_{4}(\alpha)\right)$, então $a_{1}$ e $b_{1}$ são da forma $a_{1}=\lambda b+\lambda_{1} a+\lambda_{2} a^{2}+$ $\lambda_{3} a^{3}+\lambda_{4} a^{4}$ e $b_{1}=\beta b+\beta_{1} a+\beta_{2} a^{2}+\beta_{3} a^{3}+\beta_{4} a^{4}$, e uma vez que $a_{1}$ tem nilíndice máximo, temos que

$$
\begin{aligned}
a_{1}^{2} & =\lambda^{2} b^{2}+\lambda_{1}^{2} a^{2}+2 \lambda_{1} \lambda_{2} a^{3}+2 \lambda_{1} \lambda_{3} a^{4}+\lambda_{2}^{2} a^{4} \\
& =\lambda_{1}^{2} a^{2}+2 \lambda_{1} \lambda_{2} a^{3}+\left(\lambda^{2} \alpha+2 \lambda_{1} \lambda_{3}+\lambda_{2}^{2}\right) a^{4}, \\
a_{1}^{3} & =\lambda_{1}^{3} a^{3}+2 \lambda_{1}^{2} \lambda_{2} a^{4}+\lambda_{2} \lambda_{1}^{2} a^{4}=\lambda_{1}^{3} a^{3}+3 \lambda_{1}^{2} \lambda_{2} a^{4}, \\
a_{1}^{4} & =\lambda_{1}^{4} a^{4} \neq 0,
\end{aligned}
$$

portanto $\lambda_{1} \neq 0$. Agora,

$$
\begin{aligned}
a_{1} b_{1} & =\lambda \beta b^{2}+\lambda_{1} \beta_{1} a^{2}+\lambda_{1} \beta_{2} a^{3}+\lambda_{1} \beta_{3} a^{4}+\lambda_{2} \beta_{1} a^{3}+\lambda_{2} \beta_{2} a^{4}+\lambda_{3} \beta_{1} a^{4} \\
& =\lambda_{1} \beta_{1} a^{2}+\left(\lambda_{1} \beta_{2}+\lambda_{2} \beta_{1}\right) a^{3}+\left(\lambda \beta \alpha+\lambda_{1} \beta_{3}+\lambda_{2} \beta_{2}+\lambda_{3} \beta_{1}\right) a^{4}=0
\end{aligned}
$$

$\operatorname{logo} \lambda_{1} \beta_{1}=0$ e como $\lambda_{1} \neq 0$, segue que $\beta_{1}=0$, igualmente $\beta_{2}=0$ e portanto $\beta_{3}=\frac{-\lambda \beta \alpha}{\lambda_{1}}$. Assim

$$
b_{1}=\beta b-\frac{\lambda \beta \alpha}{\lambda_{1}} a^{3}+\beta_{4} a^{4}, \quad \text { com } \quad \beta \neq 0,
$$

pois se $\beta=0$, então $b_{1}=\beta_{4} a^{4} \in \mathcal{B}^{2}$, o que não é possível. Logo

$$
b_{1}^{2}=\beta^{2} b^{2}=\beta^{2} \alpha a^{4}=\beta^{2} \frac{1}{\lambda_{1}^{4}} \alpha a_{1}^{4}
$$

e $a_{1}^{2} b_{1}=0$, portanto

$$
b_{1}^{2}=\frac{\beta^{2}}{\lambda_{1}^{4}} \alpha a_{1}^{4} \quad \text { e } \quad a_{1}^{2} b_{1}=0, \quad \text { com } \quad \lambda_{1}, \beta \neq 0,
$$

o que demonstra o lema. 
Corolário 4.11 Sejam $\alpha$ e $\alpha^{\prime}$ em $F^{*}$. Então

$$
\mathcal{B}_{4}(\alpha) \cong \mathcal{B}_{4}\left(\alpha^{\prime}\right)
$$

se, e somente se, existir um $\rho$ não nulo tal que, $\alpha^{\prime}=\alpha \rho^{2}$.

Como consequência imediata do lema anterior, temos também que para $\alpha$ diferente de zero e $\alpha^{\prime}$ em $F$,

$$
\mathcal{B}_{4}(\alpha) \approx \mathcal{B}_{3}\left(\alpha^{\prime}\right)
$$

Portanto podemos concluir de todo o anterior que $\mathcal{B}_{5} \cong \mathcal{B}_{6} \mathrm{e}$

$$
\mathcal{B}_{i} \neq \mathcal{B}_{j}, \quad(2 \leq i<j \leq 7)
$$

Passaremos agora a estudar as classes dadas por $\mathcal{B}_{1}(\alpha), \mathcal{B}_{2}(\alpha)$ e $\mathcal{B}_{3}(\alpha)$, para um $\alpha$ qualquer em $F$.

Lema 4.12 Para todo $\alpha$ e $\alpha^{\prime}$ em F,

$$
\mathcal{B}_{3}(\alpha) \cong \mathcal{B}_{3}\left(\alpha^{\prime}\right)
$$

se, e somente se, $\alpha^{\prime}=\alpha$

Prova: Seja $\alpha$ um elemento em $F$ e $(a, b) \in \mathcal{P}\left(\mathcal{B}_{3}(\alpha)\right)$ tal que $b^{2}=\alpha a^{4}$ e $a^{2} b=a^{4}$. Analisemos como são os pares $\left(a_{1}, b_{1}\right)$ de $\mathcal{P}\left(\mathcal{B}_{3}(\alpha)\right)$. Cada elemento do par anterior, exprime-se de maneira única na forma $a_{1}=\lambda b+\lambda_{1} a+\lambda_{2} a^{2}+$ $\lambda_{3} a^{3}+\lambda_{4} a^{4}$ e $b_{1}=\beta b+\beta_{1} a+\beta_{2} a^{2}+\beta_{3} a^{3}+\beta_{4} a^{4}$, e cumpre que

$$
\begin{aligned}
a_{1}^{2} & =\lambda^{2} b^{2}+2 \lambda \lambda_{2} a^{2} b+\lambda_{1}^{2} a^{2}+2 \lambda_{1} \lambda_{2} a^{3}+2 \lambda_{1} \lambda_{3} a^{4}+\lambda_{2}^{2} a^{4} \\
& =\lambda_{1}^{2} a^{2}+2 \lambda_{1} \lambda_{2} a^{3}+\left(\lambda^{2} \alpha+2 \lambda \lambda_{2}+2 \lambda_{1} \lambda_{3}+\lambda_{2}^{2}\right) a^{4}, \\
a_{1}^{3} & =\lambda \lambda_{1}^{2} a^{2} b+\lambda_{1}^{3} a^{3}+2 \lambda_{1}^{2} \lambda_{2} a^{4}+\lambda_{2} \lambda_{1}^{2} a^{4}=\lambda_{1}^{3} a^{3}+\left(\lambda \lambda_{1}^{2} \alpha+3 \lambda_{1}^{2} \lambda_{2}\right) a^{4}, \\
a_{1}^{4} & =\lambda_{1}^{4} a^{4} \neq 0,
\end{aligned}
$$


pois $a_{1}$ tem nilíndice máximo, $\operatorname{logo} \lambda_{1} \neq 0$. Como,

$$
\begin{aligned}
a_{1} b_{1}= & \lambda \beta b^{2}+\lambda \beta_{2} a^{2} b+\lambda_{1} \beta_{1} a^{2}+\lambda_{1} \beta_{2} a^{3}+\lambda_{1} \beta_{3} a^{4}+\lambda_{2} \beta a^{2} b+\lambda_{2} \beta_{1} a^{3} \\
& +\lambda_{2} \beta_{2} a^{4}+\lambda_{3} \beta_{1} a^{4} \\
= & \lambda_{1} \beta_{1} a^{2}+\left(\lambda_{1} \beta_{2}+\lambda_{2} \beta_{1}\right) a^{3}+\left(\lambda \beta \alpha+\lambda \beta_{2}+\lambda_{1} \beta_{3}+\lambda_{2} \beta+\lambda_{2} \beta_{2}\right. \\
& \left.+\lambda_{3} \beta_{1}\right) a^{4} \\
= & 0
\end{aligned}
$$

segue que, $\lambda_{1} \beta_{1}=0$ e dado que $\lambda_{1} \neq 0$, temos que $\beta_{1}=0$, igualmente $\beta_{2}=0$ e portanto $\beta_{3}=\frac{-\beta\left(\lambda \alpha+\lambda_{2}\right)}{\lambda_{1}}$. Assim

$$
b_{1}=\beta b-\frac{\beta\left(\lambda \alpha+\lambda_{2}\right)}{\lambda_{1}} a^{3}+\beta_{4} a^{4}, \quad \text { com } \quad \beta \neq 0,
$$

caso contrário $b_{1} \in \mathcal{B}^{2}$, que levaria a uma contradição. Logo

$$
b_{1}^{2}=\beta^{2} b^{2}=\beta^{2} \alpha a^{4}=\beta^{2} \frac{1}{\lambda_{1}^{4}} \alpha a_{1}^{4}
$$

$\mathrm{e}$

$$
a_{1}^{2} b_{1}=\lambda_{1}^{2} \beta a^{2} b=\lambda_{1}^{2} \beta a^{4}=\lambda_{1}^{2} \beta \frac{1}{\lambda_{1}^{4}} a_{1}^{4}
$$

Portanto

$$
b_{1}^{2}=\frac{\beta^{2}}{\lambda_{1}^{4}} \alpha a_{1}^{4} \quad \text { e } \quad a_{1}^{2} b_{1}=\frac{\beta}{\lambda_{1}^{2}} a_{1}^{4}, \quad \text { com } \quad \lambda_{1}, \beta \neq 0 .
$$

Assim, podemos concluir que dado um $\alpha^{\prime}$ de $F$, segue que $\mathcal{B}_{3}(\alpha) \cong \mathcal{B}_{3}\left(\alpha^{\prime}\right)$, se e somente se, existir $\lambda_{1}, \beta \in F^{*}$ tais que $\alpha^{\prime}=\frac{\beta^{2}}{\lambda_{1}^{4}} \alpha$ e $\frac{\beta}{\lambda_{1}^{2}}=1, \log \sigma \beta=\lambda_{1}^{2}$. Consequentemente, $\alpha^{\prime}=\frac{\beta^{2}}{\lambda_{1}^{4}} \alpha=\frac{\lambda_{1}^{4}}{\lambda_{1}^{4}} \alpha=\alpha$, o que demonstra o lema

Lema 4.13 Se $\alpha$ e $\alpha^{\prime}$ estão em $F$, então

$$
\mathcal{B}_{2}(\alpha) \cong \mathcal{B}_{2}\left(\alpha^{\prime}\right)
$$

se, e somente se, $\alpha^{\prime}=\rho^{2} \alpha$ com $\rho \in F^{*}$. 
Prova: Seja $\alpha$ um elemento de $F$. Considerar $(a, b) \in \mathcal{P}\left(\mathcal{B}_{2}(\alpha)\right)$ tal que $b^{2}=-a^{2}+\alpha a^{4}$ e $a^{2} b=a^{3}$. Se $\left(a_{1}, b_{1}\right) \in \mathcal{P}\left(\mathcal{B}_{2}(\alpha)\right)$, então podemos exprimir $a_{1}$ e $b_{1}$ sob a forma $a_{1}=\lambda b+\lambda_{1} a+\lambda_{2} a^{2}+\lambda_{3} a^{3}+\lambda_{4} a^{4}, b_{1}=\beta b+\beta_{1} a+$ $\beta_{2} a^{2}+\beta_{3} a^{3}+\beta_{4} a^{4}$. As potências de $a_{1}$ são dadas por

$$
\begin{aligned}
a_{1}^{2}= & \lambda^{2} b^{2}+2 \lambda \lambda_{2} a^{2} b+2 \lambda \lambda_{3} a^{3} b+\lambda_{1}^{2} a^{2}+2 \lambda_{1} \lambda_{2} a^{3}+2 \lambda_{1} \lambda_{3} a^{4}+\lambda_{2}^{2} a^{4} \\
= & \left(\lambda_{1}^{2}-\lambda^{2}\right) a^{2}+\left(2 \lambda \lambda_{2}+2 \lambda_{1} \lambda_{2}\right) a^{3}+\left(\lambda^{2} \alpha-2 \lambda \lambda_{3}+2 \lambda_{1} \lambda_{3}+\lambda_{2}^{2}\right) a^{4}, \\
= & \left(\lambda_{1}^{2}-\lambda^{2}\right) a^{2}+2 \lambda_{2}\left(\lambda+\lambda_{1}\right) a^{3}+\left[2 \lambda_{3}\left(\lambda_{1}-\lambda\right)+\left(\lambda^{2} \alpha+\lambda_{2}^{2}\right)\right] a^{4}, \\
a_{1}^{3}= & \lambda\left(\lambda_{1}^{2}-\lambda^{2}\right) a^{2} b+2 \lambda_{2} \lambda\left(\lambda+\lambda_{1}\right) b a^{3}+\lambda_{1}\left(\lambda_{1}^{2}-\lambda^{2}\right) a^{3}+2 \lambda_{1} \lambda_{2}\left(\lambda+\lambda_{1}\right) a^{4} \\
& +\lambda_{2}\left(\lambda_{1}^{2}-\lambda^{2}\right) a^{4} \\
= & \lambda\left(\lambda_{1}^{2}-\lambda^{2}\right) a^{3}-2 \lambda_{2} \lambda\left(\lambda+\lambda_{1}\right) a^{4}+\lambda_{1}\left(\lambda_{1}^{2}-\lambda^{2}\right) a^{3}+2 \lambda_{1} \lambda_{2}\left(\lambda+\lambda_{1}\right) a^{4} \\
& +\lambda_{2}\left(\lambda_{1}^{2}-\lambda^{2}\right) a^{4} \\
= & \left(\lambda+\lambda_{1}\right)\left(\lambda_{1}^{2}-\lambda^{2}\right) a^{3}+\left[2 \lambda_{2} \lambda^{2}-2 \lambda_{2} \lambda \lambda_{1}+2 \lambda_{1} \lambda_{2} \lambda+2 \lambda_{1}^{2} \lambda_{2}+\right. \\
& \left.\lambda_{2}\left(\lambda_{1}^{2}-\lambda^{2}\right)\right] a^{4}, \\
= & \left(\lambda+\lambda_{1}\right)\left(\lambda_{1}^{2}-\lambda^{2}\right) a^{3}+3 \lambda_{2}\left(\lambda_{1}^{2}-\lambda^{2}\right) a^{4}, \\
= & \lambda\left(\lambda+\lambda_{1}\right)\left(\lambda_{1}^{2}-\lambda^{2}\right) a^{3} b+\lambda_{1}\left(\lambda+\lambda_{1}\right)\left(\lambda_{1}^{2}-\lambda^{2}\right) a^{4} \\
= & \left(\lambda_{1}^{2}-\lambda^{2}\right)^{2} a^{4} .
\end{aligned}
$$

Como $a_{1}$ tem nilíndice máximo, temos que $\lambda_{1}^{2}-\lambda^{2} \neq 0$, portanto $\lambda_{1} \neq \pm \lambda$. Uma vez que o produto de $a_{1}$ com $b_{1}$ é zero, segue que

$$
\begin{aligned}
a_{1} b_{1}= & \lambda \beta b^{2}+\lambda \beta_{2} a^{2} b+\lambda \beta_{3} a^{3} b+\lambda_{1} \beta_{1} a^{2}+\lambda_{1} \beta_{2} a^{3}+\lambda_{1} \beta_{3} a^{4}+\lambda_{2} \beta a^{2} b \\
& +\lambda_{2} \beta_{1} a^{3}+\lambda_{2} \beta_{2} a^{4}+\lambda_{3} \beta a^{3} b+\lambda_{3} \beta_{1} a^{4} \\
= & \left(-\lambda \beta+\lambda_{1} \beta_{1}\right) a^{2}+\left(\lambda \beta_{2}+\lambda_{1} \beta_{2}+\lambda_{2} \beta+\lambda_{2} \beta_{1}\right) a^{3}+\left(\lambda \beta \alpha-\lambda \beta_{3}+\right. \\
& \left.\lambda_{1} \beta_{3}+\lambda_{2} \beta_{2}-\lambda_{3} \beta+\lambda_{3} \beta_{1}\right) a^{4} \\
= & 0 .
\end{aligned}
$$

Sendo que $\lambda_{1}^{2}-\lambda^{2} \neq 0$, cumpre-se que $\lambda_{1} \neq 0$ ou $\lambda \neq 0$. Vamos então analisar os pares $\left(a_{1}, b_{1}\right)$ nestes dois casos por separado. Combinando com Lema 4.7, podemos supor que $\lambda_{1}=1, \lambda \neq \pm 1$ ou $\lambda_{1}=0, \lambda=1$. 
- $\left(\lambda_{1}=1\right.$ e $\lambda \neq \pm 1$.) Como

$$
\begin{aligned}
a_{1} b_{1}= & \left(-\lambda \beta+\beta_{1}\right) a^{2}+\left(\lambda \beta_{2}+\beta_{2}+\lambda_{2} \beta+\lambda_{2} \beta_{1}\right) a^{3}+\left(\lambda \beta \alpha-\lambda \beta_{3}+\right. \\
& \left.\beta_{3}+\lambda_{2} \beta_{2}-\lambda_{3} \beta+\lambda_{3} \beta_{1}\right) a^{4} \\
= & 0,
\end{aligned}
$$

segue que $\beta_{1}=\lambda \beta$ e portanto $(\lambda+1) \beta_{2}=-\lambda_{2} \beta-\lambda_{2} \lambda \beta$, isto é,

$$
\beta_{2}=-\frac{\beta \lambda_{2}(\lambda+1)}{\lambda+1}=-\beta \lambda_{2}
$$

Igualmente $\beta_{3}(1-\lambda)=-\left[\lambda \beta \alpha+\lambda_{2}\left(-\beta \lambda_{2}\right)-\lambda_{3} \beta+\lambda_{3} \lambda \beta\right]$, logo

$$
\beta_{3}=-\frac{\beta\left(\lambda \alpha-\lambda_{3}+\lambda_{3} \lambda-\lambda_{2}^{2}\right)}{1-\lambda} .
$$

Assim

$b_{1}=-\lambda \beta b-\beta \lambda_{2} a^{2}-\frac{\beta\left(\lambda \alpha-\lambda_{3}+\lambda_{3} \lambda-\lambda_{2}^{2}\right)}{1-\lambda} a^{3}+\beta_{4} a^{4}, \quad$ com $\beta \neq 0$,

pois se $\beta$ fosse zero, então $b_{1}$ pertenceria a $\mathcal{B}^{2}$. Portanto

$$
\begin{aligned}
b_{1}^{2}= & \beta^{2} b^{2}-2 \lambda_{2} \beta^{2} a^{2} b+\frac{2 \beta^{2}\left(\lambda \alpha-\lambda_{2}^{2}-\lambda_{3}+\lambda_{3} \lambda\right)}{\lambda-1} a^{3} b+\lambda^{2} \beta^{2} a^{2} \\
& -2 \lambda_{2} \lambda \beta^{2} a^{3}+\frac{2 \lambda \beta^{2}\left(\lambda \alpha-\lambda_{2}^{2}-\lambda_{3}+\lambda_{3} \lambda\right)}{\lambda-1} a^{4}+\beta^{2} \lambda_{2}^{2} a^{4} \\
= & -\beta^{2} a^{2}+\alpha \beta^{2} a^{4}-2 \lambda_{2} \beta^{2} a^{3}-\frac{2 \beta^{2}\left(\lambda \alpha-\lambda_{2}^{2}-\lambda_{3}+\lambda_{3} \lambda\right)}{\lambda-1} a^{4} \\
& +\lambda^{2} \beta^{2} a^{2}-2 \lambda_{2} \lambda \beta^{2} a^{3}+\frac{2 \lambda \beta^{2}\left(\lambda \alpha-\lambda_{2}^{2}-\lambda_{3}+\lambda_{3} \lambda\right)}{\lambda-1} a^{4}+\beta^{2} \lambda_{2}^{2} a^{4} \\
= & \beta_{1}^{2}\left(\lambda^{2}-1\right) a^{2}-2 \beta^{2} \lambda_{2}(\lambda+1) a^{3}+ \\
& \beta^{2}\left[\alpha+\frac{2 \beta^{2}(\lambda-1)\left(\lambda \alpha-\lambda_{2}^{2}-\lambda_{3}+\lambda_{3} \lambda\right)}{\lambda-1}+\lambda^{2}\right] a^{4} \\
= & \beta^{2}\left(\lambda^{2}-1\right) a^{2}-2 \beta^{2} \lambda_{2}(\lambda+1) a^{3}+\beta^{2}\left(\alpha+2 \lambda \alpha-\lambda_{2}^{2}-2 \lambda_{3}+\right. \\
& \left.2 \lambda_{3} \lambda\right) a^{4},
\end{aligned}
$$


$\mathrm{e}$

$$
\begin{aligned}
a_{1}^{2} b_{1}= & \beta\left(1-\lambda^{2}\right) a^{2} b+2 \lambda_{2} \beta(\lambda+1) a^{3} b+\lambda \beta\left(1-\lambda^{2}\right) a^{3}+ \\
& 2 \lambda_{2} \lambda \beta(\lambda+1) a^{4}-\lambda_{2} \beta\left(1-\lambda^{2}\right) a^{4} \\
= & \beta\left(1-\lambda^{2}\right) a^{3}-2 \lambda_{2} \beta(\lambda+1) a^{4}+\lambda \beta\left(1-\lambda^{2}\right) a^{3}+ \\
& 2 \lambda_{2} \lambda \beta(\lambda+1) a^{4}-\lambda_{2} \beta\left(1-\lambda^{2}\right) a^{4} \\
= & \beta(1+\lambda)\left(1-\lambda^{2}\right) a^{3}+\lambda_{2} \beta\left[-2(\lambda+1)+2 \lambda(\lambda+1)-\left(1-\lambda^{2}\right)\right] a^{4} \\
= & \beta(1+\lambda)\left(1-\lambda^{2}\right) a^{3}-3 \lambda_{2} \beta\left(1-\lambda^{2}\right) a^{4} .
\end{aligned}
$$

Como precisamos escrever $b_{1}^{2}$ e $a_{1}^{2} b_{1}$ em termos das potências de $a_{1}$, utilizaremos a seguinte matriz cujas colunas correspondem as coordenadas de $a_{1}^{2}, a_{1}^{3}, a_{1}^{4}, b_{1}^{2}$ e $a_{1}^{2} b_{1}$, nessa ordem, nas potências $a^{2}, a^{3}, a^{4}$. Para facilitar a escritura seja $\theta=\alpha+2 \lambda \alpha-\lambda_{2}^{2}-2 \lambda_{3}+2 \lambda_{3} \lambda$ e $\mu=1-\lambda^{2}$. Então temos a matriz

$$
\left[\begin{array}{ccccc}
\mu & 0 & 0 & -\beta^{2} \mu & 0 \\
2 \lambda_{2}(1+\lambda) & (\lambda+1) \mu & 0 & -2 \lambda_{2} \beta^{2}(\lambda+1) & \beta(1+\lambda) \mu \\
2 \lambda_{3}(1-\lambda)+\left(\lambda^{2} \alpha+\lambda_{2}^{2}\right) & 3 \lambda_{2} \mu & \mu^{2} & \beta^{2} \theta & -3 \lambda_{2} \beta \mu
\end{array}\right],
$$

e realizando uma redução por linhas obtemos

$$
\left[\begin{array}{ccccc}
1 & 0 & 0 & -\beta^{2} & 0 \\
0 & 1 & 0 & 0 & \beta \\
0 & 0 & 1 & \frac{\alpha \beta^{2}}{(\lambda-1)^{2}} & -\frac{6 \lambda_{2} \beta}{\left(\lambda^{2}-1\right)}
\end{array}\right]
$$

Portanto, os produtos $b_{1}^{2}$ e $a_{1}^{2} b_{1}$ são da forma $-a_{1}^{2}+\alpha^{\prime} a_{1}^{4}$ e $a_{1}^{3}$ respectivamente, para algum $\alpha^{\prime} \in F$ se e somente se

$$
-\beta^{2}=-1, \quad \frac{\alpha \beta^{2}}{(\lambda-1)^{2}}=\alpha^{\prime}, \quad \beta=1 \quad \text { e }-\frac{6 \lambda_{2} \beta}{\left(\lambda^{2}-1\right)}=0 .
$$

$\operatorname{Logo} \beta=1$ e $\lambda_{2}=0$. Assim $\alpha^{\prime}=\frac{\alpha}{(\lambda-1)^{2}}$.

- $\left(\lambda=1\right.$ e $\lambda_{1}=0$.) Neste caso temos que,

$$
\begin{aligned}
a_{1} b_{1}= & -\beta a^{2}+\left(\beta_{2}+\lambda_{2} \beta+\lambda_{2} \beta_{1}\right) a^{3} \\
& +\left(\beta \alpha-\beta_{3}+\lambda_{2} \beta_{2}-\lambda_{3} \beta+\lambda_{3} \beta_{1}\right) a^{4}=0
\end{aligned}
$$


$\log \mathrm{o} \beta=0, \beta_{2}=-\beta_{1} \lambda_{2}$, e portanto $\beta_{3}=\beta_{1}\left(-\lambda_{2}^{2}+\lambda_{3}\right)$. Assim,

$$
b_{1}=\beta_{1} a-\beta_{1} \lambda_{2} a^{2}+\beta_{1}\left(-\lambda_{2}^{2}+\lambda_{3}\right) a^{3}+\beta_{4} a^{4}, \text { com } \beta_{1} \neq 0 .
$$

Logo

$$
\begin{aligned}
b_{1}^{2} & =\beta_{1}^{2} a^{2}-2 \beta_{1}^{2} \lambda_{2} a^{3}+2 \beta_{1}^{2}\left(-\lambda_{2}^{2}+\lambda_{3}\right) a^{4}+\beta_{1}^{2} \lambda_{2}^{2} a^{4} \\
& =\beta_{1}^{2} a^{2}-2 \beta_{1}^{2} \lambda_{2} a^{3}+\beta_{1}^{2}\left(-\lambda_{2}^{2}+2 \lambda_{3}\right) a^{4}
\end{aligned}
$$

e

$$
a_{1}^{2} b_{1}=-\beta_{1} a^{3}+2 \lambda_{2} \beta_{1} a^{4}+\beta_{1} \lambda_{2} a^{4}=-\beta_{1} a^{3}+3 \beta_{1} \lambda_{2} a^{4} .
$$

Portanto temos a matriz

$$
\left[\begin{array}{ccccc}
-1 & 0 & 0 & \beta_{1}^{2} & 0 \\
2 \lambda_{2} & -1 & 0 & -2 \lambda_{2} \beta_{1}^{2} & -\beta_{1} \\
-2 \lambda_{3}+\left(\alpha+\lambda_{2}^{2}\right) & -3 \lambda_{2} & 1 & \beta_{1}^{2} \theta & 3 \lambda_{2} \beta_{1}
\end{array}\right],
$$

com $\theta=\alpha-\lambda_{2}^{2}+2 \lambda_{3}$. Reduzindo a matriz por linhas obtemos

$$
\left[\begin{array}{ccccc}
1 & 0 & 0 & -\beta_{1}^{2} & 0 \\
0 & 1 & 0 & 0 & \beta_{1} \\
0 & 0 & 1 & \alpha \beta_{1}^{2} & 6 \lambda_{2} \beta_{1}
\end{array}\right] .
$$

Portanto, os produtos $b_{1}^{2}$ e $a_{1}^{2} b_{1}$ são da forma $-a_{1}^{2}+\alpha^{\prime} a_{1}^{4}$ e $a_{1}^{3}$ respectivamente, para algum $\alpha^{\prime} \in F$ se e somente se

$$
-\beta^{2}=-1, \quad \frac{\alpha \beta_{1}^{2}}{\left(\lambda_{1}-1\right)^{2}}=\alpha^{\prime}, \quad \beta=1 \quad \text { e }-\frac{6 \lambda_{2} \beta_{1}}{\lambda_{1}^{2}-1}=0 .
$$

$\operatorname{Logo} \beta=1, \lambda_{2}=0$ e assim $\alpha^{\prime}=\alpha$. Isto prova o lema.

A demonstração do próximo lema segue os mesmos pasos da demonstração do lema anterior, pelo qual omitiremos alguns detalhes. 
Lema 4.14 Para todo $\alpha$ e $\alpha^{\prime}$ em $F$,

$$
\mathcal{B}_{1}(\alpha) \cong \mathcal{B}_{1}\left(\alpha^{\prime}\right)
$$

se, e somente se, existir $\lambda_{1}$ em $F$ com $\lambda_{1} \neq \pm 1$, tal que $\alpha^{\prime}=\frac{\left(\lambda_{1}+1\right)^{4}}{\left(\lambda_{1}-1\right)^{4}} \alpha+$ $\frac{\lambda_{1}\left(\lambda_{1}^{2}+1\right)}{2\left(\lambda_{1}-1\right)^{4}}$.

Prova: Seja $\alpha$ em $F$ e $(a, b)$ um elemento de $\mathcal{P}\left(\mathcal{B}_{1}(\alpha)\right)$ que determina uma base com produtos $b^{2}=-a^{2}+a^{3}+\alpha a^{4}, a^{2} b=a^{3}$ e $a^{3} b=-a^{4}$. Todo par $\left(a_{1}, b_{1}\right) \in \mathcal{P}\left(\mathcal{B}_{1}(\alpha)\right)$, escreve-se de maneira única como $a_{1}=\lambda b+\lambda_{1} a+$ $\lambda_{2} a^{2}+\lambda_{3} a^{3}+\lambda_{4} a^{4}$ e $b_{1}=\beta b+\beta_{1} a+\beta_{2} a^{2}+\beta_{3} a^{3}+\beta_{4} a^{4}$. Calculando as potências de $a_{1}$, obtemos que

$$
\begin{aligned}
a_{1}^{2}= & \lambda^{2} b^{2}+2 \lambda \lambda_{2} a^{2} b+2 \lambda \lambda_{3} a^{3} b+\lambda_{1}^{2} a^{2}+2 \lambda_{1} \lambda_{2} a^{3}+2 \lambda_{1} \lambda_{3} a^{4}+\lambda_{2}^{2} a^{4} \\
= & \left(\lambda_{1}^{2}-\lambda^{2}\right) a^{2}+\left[\lambda^{2}+2 \lambda_{2}\left(\lambda+\lambda_{1}\right)\right] a^{3}+\left[2 \lambda_{3}\left(\lambda_{1}-\lambda\right)+\left(\lambda^{2} \alpha+\lambda_{2}^{2}\right)\right] a^{4}, \\
a_{1}^{3}= & \lambda\left(\lambda_{1}^{2}-\lambda^{2}\right) a^{2} b+\lambda\left[\lambda^{2}+2 \lambda_{2}\left(\lambda+\lambda_{1}\right)\right] a^{3} b+\lambda_{1}\left(\lambda_{1}^{2}-\lambda^{2}\right) a^{3}+\lambda_{1}\left[\lambda^{2}\right. \\
& \left.+2 \lambda_{2}\left(\lambda+\lambda_{1}\right)\right] a^{4}+\lambda_{2}\left(\lambda_{1}^{2}-\lambda^{2}\right) a^{4} \\
= & \left(\lambda+\lambda_{1}\right)\left(\lambda_{1}^{2}-\lambda^{2}\right) a^{3}+\left(\lambda_{1}-\lambda\right)\left[\lambda^{2}+3 \lambda_{2}\left(\lambda+\lambda_{1}\right)\right] a^{4}, \\
a_{1}^{4}= & \lambda\left(\lambda+\lambda_{1}\right)\left(\lambda_{1}^{2}-\lambda^{2}\right) a^{3} b+\lambda_{1}\left(\lambda+\lambda_{1}\right)\left(\lambda_{1}^{2}-\lambda^{2}\right) a^{4} \\
= & \left(\lambda_{1}^{2}-\lambda^{2}\right)^{2} a^{4},
\end{aligned}
$$

e uma vez que $a_{1}$ tem nilíndice máximo, segue que $\lambda_{1}^{2}-\lambda^{2} \neq 0$, portanto $\lambda_{1} \neq \pm \lambda$. Agora,

$$
\begin{aligned}
a_{1} b_{1}= & \lambda \beta b^{2}+\lambda \beta_{2} a^{2} b+\lambda \beta_{3} a^{3} b+\lambda_{1} \beta_{1} a^{2}+\lambda_{1} \beta_{2} a^{3}+\lambda_{1} \beta_{3} a^{4}+\lambda_{2} \beta a^{2} b \\
& +\lambda_{2} \beta_{1} a^{3}+\lambda_{2} \beta_{2} a^{4}+\lambda_{3} \beta a^{3} b+\lambda_{3} \beta_{1} a^{4} \\
= & \left(-\lambda \beta+\lambda_{1} \beta_{1}\right) a^{2}+\left(\lambda \beta+\lambda \beta_{2}+\lambda_{1} \beta_{2}+\lambda_{2} \beta+\lambda_{2} \beta_{1}\right) a^{3}+\left(\lambda \beta \alpha-\lambda \beta_{3}\right. \\
& \left.+\lambda_{1} \beta_{3}+\lambda_{2} \beta_{2}-\lambda_{3} \beta+\lambda_{3} \beta_{1}\right) a^{4} \\
= & 0 .
\end{aligned}
$$

- $\left(\lambda_{1}=1\right.$ e $\lambda \neq \pm 1$.) Dado que

$$
\begin{aligned}
a_{1} b_{1}= & \left(-\lambda \beta+\beta_{1}\right) a^{2}+\left(\lambda \beta+\lambda \beta_{2}+\beta_{2}+\lambda_{2} \beta+\lambda_{2} \beta_{1}\right) a^{3}+(\lambda \beta \alpha \\
& \left.-\lambda \beta_{3}+\beta_{3}+\lambda_{2} \beta_{2}-\lambda_{3} \beta+\lambda_{3} \beta_{1}\right) a^{4} \\
= & 0,
\end{aligned}
$$


temos que $\beta_{1}=\lambda \beta$,

$$
\beta_{2}=-\frac{\beta \lambda+\lambda_{2} \beta+\lambda_{2} \lambda \beta}{(\lambda+1)}
$$

e

$$
\begin{aligned}
\beta_{3}(1-\lambda) & =-\lambda \beta \alpha-\lambda_{2}\left[-\frac{\beta \lambda+\lambda_{2} \beta+\lambda_{2} \lambda \beta}{(\lambda+1)}\right]+\lambda_{3} \beta-\lambda_{3} \lambda \beta \\
& =-\frac{\beta\left[\left(\lambda \alpha-\lambda_{3}+\lambda_{3} \lambda\right)(\lambda+1)-\lambda_{2}\left(\lambda+\lambda_{2}+\lambda_{2} \lambda\right)\right]}{(\lambda+1)} .
\end{aligned}
$$

Assim

$$
\beta_{3}=\frac{\beta\left(\lambda^{2} \alpha+\lambda_{3} \lambda^{2}+\lambda \alpha-\lambda_{3}-\lambda_{2} \lambda-\lambda_{2}^{2}-\lambda_{2}^{2} \lambda\right)}{\lambda^{2}-1}
$$

e portanto

$$
\begin{aligned}
b_{1}= & \beta b+\lambda \beta a-\frac{\beta\left(\lambda+\lambda_{2}+\lambda_{2} \lambda\right)}{\lambda+1} a^{2} \\
& +\frac{\beta\left(\lambda^{2} \alpha+\lambda_{3} \lambda^{2}+\lambda \alpha-\lambda_{3}-\lambda_{2} \lambda-\lambda_{2}^{2}-\lambda_{2}^{2} \lambda\right)}{\lambda^{2}-1} a^{3}+\beta_{4} a^{4},
\end{aligned}
$$

com $\beta \neq 0$. Uma vez que pelo Lema 4.7, podemos considerar múltiplos escalares de $b_{1}$ e $\beta \neq 0$, podemos supor que $\beta=1$. Assim

$$
\begin{aligned}
b_{1}= & b+\lambda a-\frac{\left(\lambda+\lambda_{2}+\lambda_{2} \lambda\right)}{\lambda+1} a^{2} \\
& +\frac{\left(\lambda^{2} \alpha+\lambda_{3} \lambda^{2}+\lambda \alpha-\lambda_{3}-\lambda_{2} \lambda-\lambda_{2}^{2}-\lambda_{2}^{2} \lambda\right)}{\lambda^{2}-1} a^{3}+\beta_{4} a^{4} .
\end{aligned}
$$

Logo

$$
\begin{aligned}
b_{1}^{2}= & b^{2}-\frac{2\left(\lambda+\lambda_{2}+\lambda_{2} \lambda\right)}{(\lambda+1)} a^{2} b+\lambda^{2} a^{2}-\frac{2 \lambda\left(\lambda+\lambda_{2}+\lambda_{2} \lambda\right)}{(\lambda+1)} a^{3}+ \\
& \frac{2\left(\lambda^{2} \alpha+\lambda_{3} \lambda^{2}+\lambda \alpha-\lambda_{3}-\lambda_{2} \lambda-\lambda_{2}^{2}-\lambda_{2}^{2} \lambda\right)}{\left(\lambda^{2}-1\right)} a^{3} b+ \\
& \frac{2 \lambda\left(\lambda^{2} \alpha+\lambda_{3} \lambda^{2}+\lambda \alpha-\lambda_{3}-\lambda_{2} \lambda-\lambda_{2}^{2}-\lambda_{2}^{2} \lambda\right)}{\left(\lambda^{2}-1\right)} a^{4} \\
& +\frac{\left(\lambda+\lambda_{2}+\lambda_{2} \lambda\right)^{2}}{(\lambda+1)^{2}} a^{4}
\end{aligned}
$$




$$
\begin{gathered}
=\left(\lambda^{2}-1\right) a^{2}+\beta^{2}\left[1-\frac{2\left(\lambda+\lambda_{2}+\lambda_{2} \lambda\right)}{(\lambda+1)}-\frac{2 \lambda\left(\lambda+\lambda_{2}+\lambda_{2} \lambda\right)}{(\lambda+1)}\right] a^{3} \\
+\left[\alpha+\frac{2(\lambda-1)\left(\lambda^{2} \alpha+\lambda_{3} \lambda^{2}+\lambda \alpha-\lambda_{3}-\lambda_{2} \lambda-\lambda_{2}^{2}-\lambda_{2}^{2} \lambda\right)}{\left(\lambda^{2}-1\right)}\right. \\
\left.\quad+\frac{\left(\lambda^{2}+2 \lambda \lambda_{2}+2 \lambda^{2} \lambda_{2}+\lambda_{2}^{2}+2 \lambda_{2}^{2} \lambda+\lambda_{2}^{2} \lambda^{2}\right)}{(\lambda+1)^{2}}\right] a^{4} \\
\quad+\left(\lambda^{2}-1\right) a^{2}-\left(2 \lambda+2 \lambda_{2}+2 \lambda_{2} \lambda-1\right) a^{3}+\frac{1}{(\lambda+1)^{2}}\left[2 \lambda^{3} \alpha+2 \lambda_{3} \lambda^{3}\right. \\
+5 \lambda^{2} \alpha-2 \lambda \lambda_{3}-2 \lambda_{2}^{2} \lambda-\lambda_{2}^{2} \lambda^{2}+2 \lambda_{3} \lambda^{2}+4 \lambda \alpha-2 \lambda_{3}-\lambda_{2}^{2}+\lambda^{2} \\
+\alpha] a^{4}
\end{gathered}
$$

e

$$
\begin{aligned}
a_{1}^{2} b_{1}= & \left(1-\lambda^{2}\right) a^{2} b+\left[\lambda^{2}+2 \lambda_{2}(\lambda+1)\right] a^{3} b+\lambda\left(1-\lambda^{2}\right) a^{3}+\lambda\left[\lambda^{2}+\right. \\
& \left.2 \lambda_{2}(\lambda+1)\right] a^{4}-\frac{\left(\lambda+\lambda_{2}+\lambda_{2} \lambda\right)\left(1-\lambda^{2}\right)}{(\lambda+1)} a^{4} \\
= & \left(1-\lambda^{2}\right) a^{3}-\left[\lambda^{2}+2 \lambda_{2}(\lambda+1)\right] a^{4}+\lambda\left(1-\lambda^{2}\right) a^{3}+\lambda\left[\lambda^{2}+\right. \\
& \left.2 \lambda_{2}(\lambda+1)\right] a^{4}-\frac{\left(\lambda+\lambda_{2}+\lambda_{2} \lambda\right)\left(1-\lambda^{2}\right)}{(\lambda+1)} a^{4} \\
= & (1+\lambda)\left(1-\lambda^{2}\right) a^{3}+\left[(\lambda-1)\left(\lambda^{2}+2 \lambda_{2} \lambda+2 \lambda_{2}\right)-(1-\lambda)(\lambda+\right. \\
& \left.\left.\lambda_{2}+\lambda_{2} \lambda\right)\right] a^{4} \\
= & (1+\lambda)\left(1-\lambda^{2}\right) a^{3}+\left(\lambda^{2}-1\right)\left(\lambda+3 \lambda_{2}\right) a^{4} .
\end{aligned}
$$

Portanto temos a matriz

$$
\left[\begin{array}{ccccc}
\mu & 0 & 0 & -\mu & 0 \\
\lambda^{2}+2 \lambda_{2}(1+\lambda) & (\lambda+1) \mu & 0 & -\epsilon & (1+\lambda) \mu \\
2 \lambda_{3}(1-\lambda)+\left(\lambda_{2}^{2}+\lambda^{2} \alpha\right) & (1-\lambda)\left[\lambda^{2}+3 \lambda_{2}(1+\lambda)\right] & \mu^{2} & \frac{\theta}{(\lambda+1)^{2}} & -\mu\left(\lambda+3 \lambda_{2}\right)
\end{array}\right]
$$

$\operatorname{com} \theta=2 \lambda^{3} \alpha+2 \lambda_{3} \lambda^{3}+5 \lambda^{2} \alpha-2 \lambda \lambda_{3}-2 \lambda_{2}^{2} \lambda-\lambda_{2}^{2} \lambda^{2}+2 \lambda_{3} \lambda^{2}+4 \lambda \alpha-$ $2 \lambda_{3}-\lambda_{2}^{2}+\lambda^{2}+\alpha, \quad \epsilon=2 \lambda+2 \lambda_{2}+2 \lambda_{2} \lambda-1$ e $\mu=1-\lambda^{2}$, que reduzida por linhas da a matriz 


$$
\left[\begin{array}{ccccc}
1 & 0 & 0 & -1 & 0 \\
0 & 1 & 0 & -\frac{1(\lambda-1)}{(\lambda+1)^{2}} & 1 \\
0 & 0 & 1 & \frac{\phi}{(\lambda+1)^{2}\left(\lambda^{2}-1\right)^{2}} & \frac{\left(2 \lambda^{2}+\lambda+6 \lambda \lambda_{2}+6 \lambda_{2}\right)}{(\lambda-1)(\lambda+1)^{2}}
\end{array}\right]
$$

$\operatorname{com} \phi=\lambda^{4} \alpha-\lambda^{4}+4 \lambda^{3} \alpha-3 \lambda^{3} \lambda_{2}+2 \lambda^{3}+6 \lambda^{2} \alpha+3 \lambda^{2} \lambda_{2}+4 \lambda \alpha+3 \lambda \lambda_{2}+$ $\alpha-3 \lambda_{2}$. Portanto utilizando o Lema 4.7 , temos que os produtos $b_{1}^{2}$ e $a_{1}^{2} b_{1}$ são da forma $-a_{1}^{2}+a_{1}^{3}+\alpha^{\prime} a_{1}^{4}$ e $a_{1}^{3}$, para algum $\alpha^{\prime}$ em $F$, se e somente se

$$
\begin{gathered}
-\frac{x^{2}}{y^{3}} \frac{(\lambda-1)}{(\lambda+1)^{2}}=-1, \quad \frac{x^{2}}{y^{4}} \frac{\phi}{(\lambda+1)^{2}\left(\lambda^{2}-1\right)^{2}}=\alpha^{\prime}, \\
\frac{x}{y}=1 \text { e } \frac{\left(2 \lambda^{2}+\lambda+6 \lambda \lambda_{2}+6 \lambda_{2}\right)}{(\lambda-1)(\lambda+1)^{2}}=0,
\end{gathered}
$$

para $x$ e $y$ não nulos. Logo $x=y$ do que segue que $-\frac{1}{y} \frac{(\lambda-1)}{(\lambda+1)^{2}}=-1$, isto é, $y=\frac{(\lambda-1)}{(\lambda+1)^{2}}$. Por outro lado temos que $\lambda_{2}=-\frac{\lambda(2 \lambda+1)}{6(\lambda+1)}$ que substituída em $\phi$, nos da $\phi=(\lambda+1)^{4} \alpha+\frac{\lambda\left(\lambda^{2}+1\right)}{2}$. Consequentemente

$$
\begin{aligned}
\alpha^{\prime} & =\frac{1}{y^{2}} \frac{\phi}{(\lambda+1)^{2}\left(\lambda^{2}-1\right)^{2}}=\frac{(\lambda+1)^{4}}{(\lambda-1)^{2}} \frac{\phi}{(\lambda+1)^{2}\left(\lambda^{2}-1\right)^{2}}=\frac{\phi}{(\lambda-1)^{4}} \\
& =\frac{(\lambda+1)^{4}}{(\lambda-1)^{4}} \alpha+\frac{\lambda\left(\lambda^{2}+1\right)}{2(\lambda-1)^{4}} .
\end{aligned}
$$

- $\left(\lambda=1\right.$ e $\left.\lambda_{1}=0.\right)$ Então

$$
\begin{aligned}
a_{1} b_{1}= & -\beta a^{2}+\left(\beta+\beta_{2}+\lambda_{2} \beta+\lambda_{2} \beta_{1}\right) a^{3}+(\beta \alpha \\
& \left.-\beta_{3}+\lambda_{2} \beta_{2}-\lambda_{3} \beta+\lambda_{3} \beta_{1}\right) a^{4} \\
= & 0
\end{aligned}
$$

$\log \mathrm{o} \beta=0, \beta_{2}=-\beta_{1} \lambda_{2}$ e $\beta_{3}=\beta_{1}\left(\lambda_{3}-\lambda_{2}^{2}\right)$. Assim

$$
b_{1}=\beta_{1} a-\beta_{1} \lambda_{2} a^{2}+\beta_{1}\left(\lambda_{3}-\lambda_{2}^{2}\right) a^{3}+\beta_{4} a^{4}, \quad \text { com } \beta_{1} \neq 0,
$$


e podemos supor, igual que no caso anterior, que $\beta=1$, isto é,

$$
b_{1}=a-\lambda_{2} a^{2}+\left(\lambda_{3}-\lambda_{2}^{2}\right) a^{3}+\beta_{4} a^{4} .
$$

Portanto

$$
\begin{gathered}
b_{1}^{2}=a^{2}-2 \lambda_{2} a^{3}+\left(2 \lambda_{3}-\lambda_{2}^{2}\right) a^{4}, \\
a_{1}^{2} b_{1}=-a^{3}+\left(1+3 \lambda_{2}\right) a^{4},
\end{gathered}
$$

e temos a matriz

$$
\left[\begin{array}{ccccc}
-1 & 0 & 0 & 1 & 0 \\
1+2 \lambda_{2} & -1 & 0 & -2 \lambda_{2} & -1 \\
\alpha-2 \lambda_{3}+\lambda_{2}^{2} & -1-3 \lambda_{2} & 1 & 2 \lambda_{3}-\lambda_{2}^{2} & 1+3 \lambda_{2}
\end{array}\right],
$$

que reduzida por linhas da a matriz

$$
\left[\begin{array}{ccccc}
1 & 0 & 0 & -1 & 0 \\
0 & 1 & 0 & -1 & 1 \\
0 & 0 & 1 & \alpha-1-3 \lambda_{2} & 2+6 \lambda_{2}
\end{array}\right]
$$

Logo os produtos $b_{1}^{2}$ e $a_{1}^{2} b_{1}$ são da forma $-a_{1}^{2}+a_{1}^{3}+\alpha^{\prime} a_{1}^{4}$ e $a_{1}^{3}$, para algum $\alpha^{\prime}$ em $F$, se e somente se

$$
\alpha-1-3 \lambda_{2}=\alpha^{\prime} \quad \text { e } 2+6 \lambda_{2}=0
$$

do que segue que $\lambda_{2}=-\frac{1}{3}$ e $\operatorname{assim} \alpha=\alpha^{\prime}$.

Observamos que o resultado obtido no segundo ítem é um caso particular do primeiro para $\lambda=0$. Isto prova o lema.

Podemos finalmente enunciar o seguinte teorema, cuja prova decorre de todo o anterior. 
Teorema 4.15 Uma nilálgebra comutativa de p.a. de dimensão e nilíndice 5, é isomorfa a uma e somente uma álgebra com base $\left\{b, a, a^{2}, a^{3}, a^{4}\right\}$, e produtos não triviais e não zero conforme um dos casos que aparecem na seguinte lista:

$$
\begin{aligned}
& \mathcal{A}_{1}(\alpha): \quad b^{2}=-a^{2}+a^{3}+\alpha a^{4}, \quad a^{2} b=a^{3}, \quad a^{3} b=-a^{4} ; \\
& \mathcal{A}_{2}(\alpha): \quad b^{2}=-a^{2}+\alpha a^{4}, \quad a^{2} b=a^{3}, \quad a^{3} b=-a^{4}, \quad\left[\rho^{2}\right] ; \\
& \mathcal{A}_{3}(\alpha): \quad b^{2}=\alpha a^{4}, \quad a^{2} b=a^{4} \\
& \mathcal{A}_{4}(\alpha): \quad b^{2}=\alpha a^{4}, \\
& \mathcal{A}_{5}: \quad b^{2}=a^{3} .
\end{aligned}
$$

A condição suficiente e necessária para que $\mathcal{A}_{3}\left(\alpha^{\prime}\right)$ seja isomorfa com $\mathcal{A}_{3}(\alpha)$ é $\alpha^{\prime}=\alpha$. $E \mathcal{A}_{1}(\alpha) \cong \mathcal{A}_{1}\left(\alpha^{\prime}\right)$ se, e somente se, existir um $\lambda \neq \pm 1$ tal que $\alpha^{\prime}=\frac{\left(\lambda_{1}+1\right)^{4}}{\left(\lambda_{1}-1\right)^{4}} \alpha+\frac{\lambda_{1}\left(\lambda_{1}^{2}+1\right)}{2\left(\lambda_{1}-1\right)^{4}}$.

\subsection{Classificação para dimensão seis.}

Classificaremos, a menos de isomorfismos, as nilálgebras comutativas $\mathcal{B}$ de p.a. de dimensão e nilíndice 6. Iniciaremos demonstrando o seguinte lema, que dá os produtos para uma base determinada pelo par $(a, b)$ em $\mathcal{P}(\mathcal{B})$.

Teorema 4.16 Seja $\mathcal{B}$ uma nilálgebra comutativa de p.a. de dimensão e nilíndice 6 e $(a, b) \in \mathcal{P}(\mathcal{B})$. Então para a base determinada pelo par $(a, b)$, os produtos não triviais são dados por:

$$
\begin{aligned}
b^{2} & =-\gamma_{3}^{2} a^{2}+\alpha_{4} a^{4}+\alpha_{5} a^{5}, \\
a^{2} b & =\gamma_{3} a^{3}+\gamma_{4} a^{4}+\gamma_{5} a^{5}, \\
a^{3} b & =-\gamma_{3} a^{4}-\gamma_{4} a^{5}, \\
a^{4} b & =\gamma_{3} a^{5} .
\end{aligned}
$$

Reciprocamente, se $\mathcal{B}$ é uma nilálgebra comutativa, com base $\left\{b, a, a^{2}, a^{3}, a^{4}, a^{5}\right\}$, 
e produtos $a b=a^{k}=0, a^{i} a^{j}=a^{i+j}$, para todo $k \geq 6$ e $i, j \geq 1$, e (4.7), então $\mathcal{B}$ é de p.a. e nilíndice 6.

Prova: Seja $\mathcal{B}$ uma nilálgebra comutativa de p.a. de dimensão e nilíndice 6 , e base e produtos como no enunciado do lema. Uma vez que $A_{a}^{5}=\left\langle a^{5}\right\rangle$ é um ideal de $\mathcal{B}$, temos que $\overline{\mathcal{B}}=\mathcal{B} / A_{a}^{5}$ é uma nilálgebra comutativa de p.a de dimensão e nilíndice 5 , com base $\left\{\bar{b}, \bar{a}, \bar{a}^{2}, \bar{a}^{3}, \bar{a}^{4}\right\}$ e produtos como indicados no Teorema 4.6. Logo $\bar{b}^{2}=-\gamma_{3}^{2} \bar{a}^{2}+\alpha_{3} \bar{a}^{3}+\alpha_{4} \bar{a}^{4}$ e $a^{2} b=\gamma_{3} \bar{a}^{3}+\gamma_{4} \bar{a}^{4}$. Portanto

$$
b^{2}=-\gamma_{3}^{2} a^{2}+\alpha_{3} a^{3}+\alpha_{4} a^{4}+\alpha_{5} a^{5} \quad \text { e } \quad a^{2} b=\gamma_{3} a^{3}+\gamma_{4} a^{4}+\gamma_{5} a^{5} .
$$

Por (4.2), temos que

$$
a^{3} b=-\gamma_{3} a^{4}-\gamma_{4} a^{5}
$$

e por (4.3),

$$
a^{4} b=\frac{1}{3}\left[3\left(\gamma_{3} a^{5}\right)-2\left(-\gamma_{3} a^{5}\right)-2\left(\gamma_{3} a^{5}\right)\right]=\gamma_{3} a^{5} .
$$

Temos também que

$$
a^{2} b^{2}=-\gamma_{3}^{2} a^{4}+\alpha_{3} a^{5}
$$

e por (4.1),

$$
\begin{aligned}
a^{2} b^{2} & =\frac{1}{2}\left[\left(-\gamma_{3} a^{4}+\alpha_{3} a^{5}\right)+\left(\gamma_{3} a^{3} b+\gamma_{4} a^{4} b\right)\right] \\
& =\frac{1}{2}\left[-\gamma_{3}^{2} a^{4}+\alpha_{3} a^{5}-\gamma_{3}^{2} a^{4}-\gamma_{3} \gamma_{4} a^{5}+\gamma_{4} \gamma_{3} a^{5}\right] \\
& =\frac{1}{2}\left[-2 \gamma_{3}^{2} a^{4}+\alpha_{3} a^{5}\right]
\end{aligned}
$$

$\operatorname{logo} \alpha_{3}=0$. Assim

$$
b^{2}=-\gamma_{3}^{2} a^{2}+\alpha_{4} a^{4}+\alpha_{5} a^{5},
$$

o que demonstra a primeira parte do teorema. Para a outra, seja $\mathcal{B}$ com base $\left\{b, a, a^{2}, a^{3}, a^{4}, a^{5}\right\}$ e produtos dados por $a b=a^{k}=0, a^{i} a^{j}=a^{i+j}$, para $k \geq$ 6 e $i, j \geq 1$, e (4.7). Então se $x=\lambda b+\sum_{i=1}^{5} \lambda_{i} a^{i}$, temos que 


$$
\begin{aligned}
x^{2}= & \left(-\lambda^{2} \gamma_{3}^{2}+\lambda_{1}^{2}\right) a^{2}+\left(2 \lambda \lambda_{2} \alpha_{3}+2 \lambda_{1} \lambda_{2}\right) a^{3}+\left(\lambda^{2} \alpha_{4}+2 \lambda \lambda_{2} \gamma_{4}-2 \lambda \lambda_{3} \gamma_{3}\right. \\
& \left.+2 \lambda_{1} \lambda_{3}+\lambda_{2}^{2}\right) a^{4}+\left(\lambda^{2} \alpha_{5}+2 \lambda \lambda_{2} \gamma_{5}-2 \lambda \lambda_{3} \gamma_{4}+2 \lambda \lambda_{4} \gamma_{3}+2 \lambda_{1} \lambda_{4}+\right. \\
& \left.2 \lambda_{2} \lambda_{3}\right) a^{5}, \\
x^{3}= & \left(-\lambda^{3} \gamma_{3}^{3}+\lambda \lambda_{1}^{2} \gamma_{3}-\lambda_{1} \lambda^{2} \gamma_{3}^{2}+\lambda_{1}^{3}\right) a^{3}+\left(-\lambda^{3} \gamma_{3}^{2} \gamma_{4}+\lambda \lambda_{1}^{2} \gamma_{4}-3 \lambda^{2} \lambda_{2} \gamma_{3}^{2}\right. \\
& \left.+3 \lambda_{1}^{2} \lambda_{2}\right) a^{4}+\left(-\lambda^{3} \gamma_{3}^{2} \gamma_{5}+\lambda \lambda_{1}^{2} \gamma_{5}+\lambda^{3} \gamma_{3} \alpha_{4}-3 \lambda^{2} \lambda_{3} \gamma_{3}^{2}+3 \lambda \lambda_{2}^{2} \gamma_{3}\right. \\
& \left.+\lambda^{2} \lambda_{1} \alpha_{4}+3 \lambda_{1}^{2} \lambda_{3}+3 \lambda_{1} \lambda_{2}^{2}\right) a^{5}, \\
x^{4}= & \left(\lambda^{4} \gamma_{3}^{4}-2 \lambda^{2} \lambda_{1}^{2} \gamma_{3}^{2}+\lambda_{1}^{4}\right) a^{4}+\left(-4 \lambda^{3} \lambda_{2} \gamma_{3}^{3}+4 \lambda \lambda_{1}^{2} \lambda_{2} \gamma_{3}-4 \lambda^{2} \lambda_{1} \lambda_{2} \gamma_{3}^{2}\right. \\
& \left.+4 \lambda_{1}^{3} \lambda_{2}\right) a^{5}=\left(\lambda^{2} \gamma_{3}^{2}-\lambda_{1}^{2}\right)^{2} a^{4}-4 \lambda_{2}\left(\lambda^{2} \gamma_{3}^{2}-\lambda_{1}^{2}\right)\left(\lambda \gamma_{3}+\lambda_{1}\right) a^{5}, \\
x^{5}= & \left(\lambda^{5} \gamma_{3}^{5}-2 \lambda^{3} \lambda_{1}^{2} \gamma_{3}^{3}+\lambda \lambda_{1}^{4} \gamma_{3}+\lambda^{4} \lambda_{1} \gamma_{3}^{4}-2 \lambda^{2} \lambda_{1}^{3} \gamma_{3}^{2}+\lambda_{1}^{5}\right) a^{5}, \\
x^{6}= & 0, \\
x^{2} x^{2}= & \left(\lambda^{2} \gamma_{3}^{2}-\lambda_{1}^{2}\right)^{2} a^{4}-4 \lambda_{2}\left(\lambda^{2} \gamma_{3}^{2}-\lambda_{1}^{2}\right)\left(\lambda \gamma_{3}+\lambda_{1}\right) a^{5},
\end{aligned}
$$

$\operatorname{logo} x^{4}=x^{2} x^{2}$, ficando demonstrado que $\mathcal{B}$ é uma nilálgebra comutativa de p.a. de dimensão e nilíndice 6 .

Utilizaremos a notação $\mathcal{B}\left(\alpha_{4}, \alpha_{5}, \gamma_{3}, \gamma_{4}, \gamma_{5}\right)$, para nos referir a álgebra do enunciado do lema anterior. A matriz coordenada por linhas, do operador $L_{b}$, na base $\left\{b, a, a^{2}, a^{3}, a^{4}, a^{5}\right\}$ é

$$
\left(\begin{array}{cccccc}
0 & 0 & -\gamma_{3}^{2} & 0 & \alpha_{4} & \alpha_{5} \\
0 & 0 & 0 & 0 & 0 & 0 \\
0 & 0 & 0 & \gamma_{3} & \gamma_{4} & \gamma_{5} \\
0 & 0 & 0 & 0 & -\gamma_{3} & -\gamma_{4} \\
0 & 0 & 0 & 0 & 0 & \gamma_{3} \\
0 & 0 & 0 & 0 & 0 & 0
\end{array}\right)
$$

A continuação, estudaremos as classes de isomorfismos das álgebras da forma $\mathcal{B}\left(\alpha_{4}, \alpha_{5}, \gamma_{3}, \gamma_{4}, \gamma_{5}\right)$. 
Lema 4.17 Para todo $\lambda$ e $\beta$ em $F^{*}$,

$$
\mathcal{B}\left(\alpha_{4}, \alpha_{5}, \gamma_{3}, \gamma_{4}, \gamma_{5}\right) \cong \mathcal{B}\left(\frac{\beta^{2}}{\lambda^{4}} \alpha_{4}, \frac{\beta^{2}}{\lambda^{5}} \alpha_{5}, \frac{\beta}{\lambda} \gamma_{3}, \frac{\beta}{\lambda^{2}} \gamma_{4}, \frac{\beta}{\lambda^{3}} \gamma_{5}\right)
$$

Prova: Seja $\mathcal{B}$ a álgebra $\mathcal{B}\left(\alpha_{4}, \alpha_{5}, \gamma_{3}, \gamma_{4}, \gamma_{5}\right)$, com base determinada pelo par $(a, b)$. Para $\lambda$ e $\beta$ não nulos definimos os elementos $a_{1}:=\lambda a$ e $b_{1}:=$ $\beta b$. É claro que $\left(a_{1}, b_{1}\right)$ pertence a $\mathcal{P}(\mathcal{B})$. Determinemos as constantes de estrutura de $\mathcal{B}$ em relação a base determinada por $\left(a_{1}, b_{1}\right)$. Como $a_{1}^{2}=\lambda^{2} a^{2}$, $a_{1}^{3}=\lambda^{3} a^{3}, \quad a_{1}^{4}=\lambda^{4} a^{4}$ e $a_{1}^{5}=\lambda^{5} a^{5}$, segue que

$$
\begin{aligned}
b_{1}^{2} & =\beta^{2} b^{2}=-\beta^{2} \gamma_{3}^{2} \frac{1}{\lambda^{2}} a_{1}^{2}+\beta^{2} \alpha_{4} \frac{1}{\lambda^{4}} a_{1}^{4}+\beta^{2} \alpha_{5} \frac{1}{\lambda^{5}} a_{1}^{5}, \\
a_{1}^{2} b_{1} & =\lambda^{2} \beta a^{2} b=\beta \gamma_{3} \frac{1}{\lambda} a_{1}^{3}+\beta \gamma_{4} \frac{1}{\lambda^{2}} a_{1}^{4}+\beta \gamma_{5} \frac{1}{\lambda^{3}} a_{1}^{5} .
\end{aligned}
$$

o que demonstra o lema.

\section{Lema 4.18 Seja $\rho$ em F. Então}

$$
\begin{aligned}
& \mathcal{B}\left(\alpha_{4}, \alpha_{5}, \gamma_{3}, \gamma_{4}, \gamma_{5}\right) \cong \mathcal{B}\left(\alpha_{4}, \alpha_{5}-\theta, \gamma_{3}, \gamma_{4}-6 \rho \gamma_{3}, \gamma_{5}+7 \rho\left(3 \rho \gamma_{3}-\gamma_{4}\right)\right), \\
& \operatorname{com} \quad \theta=2 \rho\left[2 \alpha_{4}-\gamma_{4}^{2}+2 \gamma_{3}\left(\gamma_{5}-\rho \gamma_{4}+\rho^{2} \gamma_{3}\right)\right] .
\end{aligned}
$$

Prova: Seja $\mathcal{B}$ a álgebra $\mathcal{B}\left(\alpha_{4}, \alpha_{5}, \gamma_{3}, \gamma_{4}, \gamma_{5}\right)$ com base $\left\{b, a, a^{2}, a^{3}, a^{4}, a^{5}\right\}$. Sejam $\rho$ em $F$,

$$
a_{1}:=a+\rho a^{2} \quad \text { e } b_{1}:=b-\rho \gamma_{3} a^{2}-\rho\left(\gamma_{4}-\rho \gamma_{3}\right) a^{3}-\rho\left[\gamma_{5}-\rho\left(\gamma_{4}-\rho \gamma_{3}\right)\right] a^{4} .
$$

Então $a_{1}^{2}=a^{2}+2 \rho a^{3}+\rho^{2} a^{4}, a_{1}^{3}=a^{3}+3 \rho a^{4}+3 \rho^{2} a^{5}, a_{1}^{4}=a^{4}+4 \rho a^{5}$, $a_{1}^{5}=a^{5} \neq 0 \mathrm{e}$

$$
\begin{aligned}
a_{1} b_{1}= & -\rho \gamma_{3} a^{3}-\rho\left(\gamma_{4}-\rho \gamma_{3}\right) a^{4}-\rho\left[\gamma_{5}-\rho\left(\gamma_{4}-\rho \gamma_{3}\right)\right] a^{5}+\rho a^{2} b-\rho^{2} \gamma_{3} a^{4} \\
& -\rho^{2}\left(\gamma_{4}-\rho \gamma_{3}\right) a^{5} \\
= & -\rho \gamma_{3} a^{3}+\left[\rho\left(\rho \gamma_{3}-\gamma_{4}\right)-\rho^{2} \gamma_{3}\right] a^{4}+\left[-\rho \gamma_{5}+\rho^{2}\left(\gamma_{4}-\rho \gamma_{3}\right)\right. \\
& \left.-\rho^{2}\left(\gamma_{4}-\rho \gamma_{3}\right)\right] a^{5}+\rho\left(\gamma_{3} a^{3}+\gamma_{4} a^{4}+\gamma_{5} a^{5}\right) \\
= & \left(-\rho \gamma_{3}+\rho \gamma_{3}\right) a^{3}+\left(-\rho \gamma_{4}+\rho \gamma_{4}\right) a^{4}+\left(-\rho \gamma_{5}+\rho \gamma_{5}\right) a^{5} \\
= & 0 .
\end{aligned}
$$


Uma vez que fica claro da definição que $b_{1} \notin A_{a_{1}}$, temos que $\left(a_{1}, b_{1}\right) \in \mathcal{P}(\mathcal{B})$. Como

$$
\begin{aligned}
b_{1}^{2}= & b^{2}-2 \rho \gamma_{3} a^{2} b-2 \rho\left(\gamma_{4}-\rho \gamma_{3}\right) a^{3} b+\rho^{2} \gamma_{3}^{2} a^{4}+2 \rho^{2} \gamma_{3}\left(\gamma_{4}-\rho \gamma_{3}\right) a^{5}- \\
& 2 \rho\left[\gamma_{5}-\rho\left(\gamma_{4}-\rho \gamma_{3}\right)\right] a^{4} b \\
= & -\gamma_{3}^{2} a^{2}-2 \rho \gamma_{3}^{2} a^{3}+\left(\alpha_{4}-\rho^{2} \gamma_{3}^{2}\right) a^{4}+\left[\alpha_{5}+2 \rho\left(\gamma_{4}^{2}-2 \gamma_{3} \gamma_{5}+\rho \gamma_{3} \gamma_{4}-\right.\right. \\
& \left.\left.2 \rho^{2} \gamma_{3}^{2}\right)\right] a^{5}
\end{aligned}
$$

$\mathrm{e}$

$$
\begin{aligned}
a_{1}^{2} b_{1} & =b a^{2}-\rho \gamma_{3} a^{4}-\rho\left(\gamma_{4}-\rho \gamma_{3}\right) a^{5}+2 \rho a^{3} b-2 \rho^{2} \gamma_{3} a^{5}+\rho^{2} a^{4} b \\
& =\gamma_{3} a^{3}+\left(\gamma_{4}-3 \rho \gamma_{3}\right) a^{4}+\left(\gamma_{5}-3 \rho \gamma_{4}\right) a^{5},
\end{aligned}
$$

temos a seguinte matriz, cujas colunas são as coordenadas de $a_{1}^{2}, \ldots, a_{1}^{5}, b_{1}^{2}$ e $a_{1}^{2} b_{1}$ em relação a $a^{2}, \ldots, a^{5}$,

$$
\left[\begin{array}{cccccc}
1 & 0 & 0 & 0 & -\gamma_{3}^{2} & 0 \\
2 \rho & 1 & 0 & 0 & -2 \rho \gamma_{3}^{2} & \gamma_{3} \\
\rho^{2} & 3 \rho & 1 & 0 & \alpha_{4}-\rho^{2} \gamma_{3}^{2} & \gamma_{4}-3 \rho \gamma_{3} \\
0 & 3 \rho^{2} & 4 \rho & 1 & \alpha_{5}+2 \rho\left(\gamma_{4}^{2}-2 \gamma_{3} \gamma_{5}+\rho \gamma_{3} \gamma_{4}-2 \rho^{2} \gamma_{3}^{2}\right) & \gamma_{5}-3 \rho \gamma_{4}
\end{array}\right]
$$

que reduzida por linhas nos leva a matriz

$$
\left[\begin{array}{cccccc}
1 & 0 & 0 & 0 & -\gamma_{3}^{2} & 0 \\
0 & 1 & 0 & 0 & 0 & \gamma_{3} \\
0 & 0 & 1 & 0 & \alpha_{4} & \gamma_{4}-6 \rho \gamma_{3} \\
0 & 0 & 0 & 1 & \alpha_{5}-2 \rho\left(2 \alpha_{4}+2 \rho \gamma_{3} \gamma_{5}-2 \rho \gamma_{3} \gamma_{4}-\gamma_{4}^{4}+2 \rho^{2} \gamma_{3}^{2}\right) & \gamma_{5}+21 \rho^{2} \gamma_{3}-7 \rho \gamma_{4}
\end{array}\right]
$$

que nos da as constantes de estrutura de $\mathcal{B}$ em relação a base determinada pelo par $\left(a_{1}, b_{1}\right)$, demonstrando o lema.

Observamos que pelo Lema 4.17 , se $\gamma_{3} \neq 0$, então tomando $\beta=\frac{1}{\gamma_{3}}$ e $\lambda=1$, obtemos que

$$
\mathcal{B}\left(\alpha_{4}, \alpha_{5}, \gamma_{3}, \gamma_{4}, \gamma_{5}\right) \cong \mathcal{B}\left(\frac{\alpha_{4}}{\gamma_{3}^{2}}, \frac{\alpha_{5}}{\gamma_{3}^{2}}, 1, \frac{\gamma_{4}}{\gamma_{3}}, \frac{\gamma_{5}}{\gamma_{3}}\right) .
$$


Portanto podemos supor, a menos de isomorfismos, que $\gamma_{3}=0$ ou $\gamma_{3}=1$. Passemos ao análise destes dois casos.

- Caso 1: $\left(\gamma_{3}=0\right.$.) Temos a álgebra $\mathcal{B}\left(\alpha_{4}, \alpha_{5}, 0, \gamma_{4}, \gamma_{5}\right)$, que pelo Lema 4.18, é isomorfa a álgebra $\mathcal{B}\left(\alpha_{4}, \alpha_{5}-2 \rho\left(2 \alpha_{4}-\gamma_{4}^{2}\right), 0, \gamma_{4}, \gamma_{5}-7 \rho \gamma_{4}\right)$, para todo $\rho$ em $F$.

- Se $\gamma_{4} \neq 0$, então fazendo $\rho=\frac{\gamma_{5}}{7 \gamma_{4}}$, temos que $\mathcal{B}\left(\alpha_{4}, \alpha_{5}, 0, \gamma_{4}, \gamma_{5}\right)$, é isomorfa à álgebra $\mathcal{B}\left(\alpha_{4}, \alpha_{5}^{\prime}, 0, \gamma_{4}, 0\right)$. Por outro lado, temos do Lema 4.7, que se $\beta=\lambda=\gamma_{4} \neq 0$, então $\mathcal{B}\left(\alpha_{4}, \alpha_{5}^{\prime}, 0, \gamma_{4}, 0\right)$, é isomorfa à álgebra $\mathcal{B}\left(\bar{\alpha}_{4}, \bar{\alpha}_{5}, 0,1,0\right)$. Se $\bar{\alpha}_{5} \neq 0$, então tomando $\lambda=\bar{\alpha}_{5}$ e $\beta=\bar{\alpha}_{5}^{2}$, obtemos que

$$
\mathcal{B}\left(\alpha_{4}, \alpha_{5}^{\prime}, 0,1,0\right) \cong \mathcal{B}\left(\bar{\alpha}_{4}, 1,0,1,0\right),
$$

e se $\bar{\alpha}_{5}=0$, então temos a álgebra $\mathcal{B}\left(\alpha_{4}, 0,0,1,0\right)$.

- Se $\gamma_{4}=0$, temos a álgebra $\mathcal{B}\left(\alpha_{4}, \alpha_{5}, 0,0, \gamma_{5}\right)$, isomorfa à álgebra $\mathcal{B}\left(\alpha_{4}, \alpha_{5}-4 \rho \alpha_{4}, 0,0, \gamma_{5}\right)$.

* Se $\alpha_{4} \neq 0$, então considerando $\rho=\frac{\alpha_{5}}{4 \alpha_{4}}$, obtemos a álgebra $\mathcal{B}\left(\alpha_{4}, 0,0,0, \gamma_{5}\right)$.

- Se $\gamma_{5} \neq 0$, então fazendo $\lambda=1$ e $\beta=\frac{1}{\gamma_{5}}$, no Lema 4.17, obtemos o isomorfismo com a álgebra $\mathcal{B}\left(\frac{\alpha_{4}}{\gamma_{5}^{2}}, 0,0,0,1\right)$. As$\operatorname{sim}$

$$
\mathcal{B}\left(\alpha_{4}, 0,0,0, \gamma_{5}\right) \cong \mathcal{B}\left(\alpha_{4}^{\prime}, 0,0,0,1\right), \quad \text { com } \quad \alpha_{4}^{\prime} \neq 0
$$

- Se $\gamma_{5}=0$, temos a álgebra $\mathcal{B}\left(\alpha_{4}, 0,0,0,0\right)$.

* Se $\alpha_{4}=0$, temos a álgebra $\mathcal{B}\left(0, \alpha_{5}, 0,0, \gamma_{5}\right)$.

- Se $\gamma_{5} \neq 0$, então fazendo $\lambda=1$ e $\beta=\frac{1}{\gamma_{5}}$, no Lema 4.17, obtemos o isomorfismo com a álgebra $\mathcal{B}\left(0, \alpha_{5}^{\prime}, 0,0,1\right)$. Se $\alpha_{5}^{\prime} \neq 0$, então considerando $\lambda=\frac{1}{\alpha_{5}^{\prime}}$ e $\beta=\frac{1}{\alpha_{5}^{\prime 3}}$, obtemos que

$$
\mathcal{B}\left(0, \alpha_{5}^{\prime}, 0,0,1\right) \cong \mathcal{B}(0,1,0,0,1),
$$

e se $\alpha_{5}^{\prime}=0$, temos a álgebra $\mathcal{B}(0,0,0,0,1)$. 
- Se $\gamma_{5}=0$ e $\alpha_{5} \neq 0$, basta tomar $\lambda=\alpha_{5}$ e $\beta=\alpha_{5}^{2}$, no Lema 4.17, para obtermos a álgebra $\mathcal{B}(0,1,0,0,0)$.

- Caso 2: $\left(\gamma_{3}=1\right.$.) Temos a álgebra $\mathcal{B}\left(\alpha_{4}, \alpha_{5}, 1, \gamma_{4}, \gamma_{5}\right)$, que pelo Lema 4.18, é isomorfa à álgebra $\mathcal{B}\left(\alpha_{4}, \alpha_{5}-\theta, 1, \gamma_{4}-6 \rho, \gamma_{5}+7 \rho\left(3 \rho-\gamma_{4}\right)\right)$, para todo $\rho$ em $F$, com $\theta=2 \rho\left(2 \alpha_{4}-\gamma_{4}^{2}+2 \gamma_{5}-2 \rho \gamma_{4}+2 \rho^{2}\right)$. Logo considerando $\rho=\frac{1}{6 \gamma_{4}}$, obtemos que

$$
\mathcal{B}\left(\alpha_{4}, \alpha_{5}, 1, \gamma_{4}, \gamma_{5}\right) \cong \mathcal{B}\left(\alpha_{4}, \alpha_{5}^{\prime}, 1,0, \gamma_{5}^{\prime}\right)
$$

Podemos concluir, de todo o anterior que, se $\mathcal{B}$ é uma nilálgebra comutativa de p.a. de dimensão e nilíndice 6 , então existe uma base da forma $\left\{b, a, a^{2}, a^{3}, a^{4}, a^{5}\right\}$, com produtos não triviais não zero, dados por um da seguinte lista, $\operatorname{com} \alpha, \theta$ e $\gamma$ em $F$.

$\mathcal{B}_{1}(\alpha, \theta, \gamma): b^{2}=-a^{2}+\alpha a^{4}+\theta a^{5}, \quad a^{2} b=a^{3}+\gamma a^{5}, \quad a^{3} b=-a^{4}, \quad a^{4} b=a^{5} ;$

$\mathcal{B}_{2}(\alpha): \quad b^{2}=\alpha a^{4}+a^{5}, \quad a^{2} b=a^{4}, \quad a^{3} b=-a^{5} ;$

$\mathcal{B}_{3}(\alpha): \quad b^{2}=\alpha a^{4}, \quad a^{2} b=a^{4}, \quad a^{3} b=-a^{5} ;$

$\mathcal{B}_{4}(\alpha): \quad b^{2}=\alpha a^{4}, \quad \quad a^{2} b=a^{5}, \quad \alpha \neq 0 ;$

$\mathcal{B}_{5}(\alpha): \quad b^{2}=\alpha a^{4}, \quad \alpha \neq 0$

$\mathcal{B}_{6}: \quad b^{2}=a^{5}, \quad a^{2} b=a^{5} ;$

$\mathcal{B}_{7}: \quad a^{2} b=a^{5} ;$

$\mathcal{B}_{8}: \quad b^{2}=a^{5}$

$\mathcal{B}_{9}:$

Uma vez que toda álgebra desta classe é isomorfa a alguma da lista anterior, resta analisar as condições de isomorfismo entre elas. É claro que

$$
\mathcal{B}_{9} \nsucceq \mathcal{B}_{i}, \quad \text { para } i \neq 9,
$$

pois a dimensão do anulador de $\mathcal{B}_{9}$ é 2 e a dimensão do anulador de $\mathcal{B}_{i}$ é 1 , para todo $i$ diferente de 9 . 
Seja $\overline{\mathcal{B}}_{i}=\mathcal{B}_{i} / \operatorname{Anul}\left(\mathcal{B}_{i}\right)$. Como a dimensão do anulador de $\overline{\mathcal{B}}_{i}$ é 1 para $i=1,2,3,4,5$, enquanto que a dimensão do anulador de $\overline{\mathcal{B}}_{j}$ é 2 para $j=$ $6,7,8$, temos as oito primeiras álgebras da lista anterior separadas em dois conjuntos de álgebras não isomorfas. O primeiro dele formado pelas álgebras $\mathcal{B}_{1}, \mathcal{B}_{2}, \mathcal{B}_{3}, \mathcal{B}_{4}, \mathcal{B}_{5}$ e o segundo formado pelas álgebras $\mathcal{B}_{6}, \mathcal{B}_{7}, \mathcal{B}_{8}$.

Observamos que a álgebra $\overline{\mathcal{B}_{1}}$ corresponde à álgebra $\mathcal{A}_{2}$, as álgebras $\overline{\mathcal{B}_{2}} \mathrm{e}$ $\overline{\mathcal{B}}_{3}$ correspondem à álgebra $\mathcal{A}_{3}$ e as álgebras $\overline{\mathcal{B}}_{4}$ e $\overline{\mathcal{B}}_{5}$ correspondem à álgebra $\mathcal{A}_{4}$, do Teorema 4.15, que não são isomorfas. Isto nos divide o primeiro conjunto em três subconjuntos de álgebras não isomorfas, sendo que no primeiro só fica a álgebra $\mathcal{B}_{1}$, portanto

$$
\mathcal{B}_{1} \not \mathcal{B}_{i}, \quad \text { para } i \neq 1 \text {. }
$$

Assim a nossa lista, sem $\mathcal{B}_{1}$ e $\mathcal{B}_{9}$, fica divida em três conjuntos de álgebras não isomorfas, mais explicitamente estes conjuntos são:

$$
C 1: \mathcal{B}_{2}(\alpha), \mathcal{B}_{3}(\alpha) . \quad C 2: \mathcal{B}_{4}(\alpha), \mathcal{B}_{5}(\alpha) . \quad C 3: \mathcal{B}_{6}, \mathcal{B}_{7}, \mathcal{B}_{8}
$$

Vejamos que as álgebras de $C 2$ são isomorfas.

Lema 4.19 Para todo $\alpha$ em $F^{*}$,

$$
\mathcal{B}_{4}(\alpha) \cong \mathcal{B}_{5}(\alpha)
$$

Prova: Seja $\alpha$ não nulo em $F$ e $\left\{b, a, a^{2}, a^{3}, a^{4}, a^{5}\right\}$ uma base para $\mathcal{B}_{5}(\alpha)$ na qual $b^{2}=\alpha a^{4}$ e os outros produtos não triviais são zero. Definamos os seguintes elementos

$$
a_{1}=-\frac{1}{\alpha} b+a \quad \text { e } \quad b_{1}=b+a^{3} .
$$

Vejamos que $\left(a_{1}, b_{1}\right)$ pertencem a $\mathcal{P}\left(\mathcal{B}_{5}(\alpha)\right)$. De fato

$$
a_{1} b_{1}=-\frac{1}{\alpha} b^{2}+a^{4}=-\frac{1}{\alpha} \alpha a^{4}+a^{4}=0
$$

e $a$ tem nilíndice máximo, pois $a_{1}^{2}=a^{2}+\frac{1}{\alpha} a^{4}, a_{1}^{3}=a^{3}+\frac{1}{\alpha} a^{5}, a_{1}^{4}=a^{4} \quad$ e $a_{1}^{5}=a^{5} \neq 0$. 
Como $b_{1}^{2}=b^{2}=\alpha a^{4}=\alpha a_{1}^{4}$ e $a_{1}^{2} b_{1}=a^{2} b=a^{5}=a_{1}^{5}$, o lema fica provado.

Assim

$$
\mathcal{B}_{4} \nsucceq \mathcal{B}_{i}, \quad \text { para } i \neq 4,5 \text {. }
$$

Provaremos agora que as álgebras do $C 3$ não são isomorfas. Para isto, determinaremos as constantes de estrutura de uma nilálgebra comutativa $\mathcal{B}$ de p.a de dimensão e nilíndice 6 , em relação a base determinada pelo par $\left(a_{1}, b_{1}\right)$ em $\mathcal{P}(\mathcal{B})$, sendo que os produtos respeito a base determinada pelo par $(a, b)$ em $\mathcal{P}(\mathcal{B})$, são $b^{2}=\theta_{1} a^{5}$ e $a^{2} b=\theta_{2} a^{5}$. Pois cada uma das álgebras de $C 3$, esta determinada pelos valores de $\theta_{1}$ e $\theta_{2}$ no conjunto $\{0,1\}$.

Lema 4.20 Seja $\mathcal{B}$ uma nilálgebra comutativa de p.a. de dimensão e nilíndice 6, com base $\left\{b, a, a^{2}, a^{3}, a^{4}, a^{5}\right\}$ tal que $b^{2}=\theta_{1} a^{5}$ e $a^{2} b=\theta_{2} a^{5}$ e os outros produtos não triviais são zero. Então para cada par $\left(a_{1}, b_{1}\right) \in \mathcal{P}(\mathcal{B})$,

$$
b_{1}^{2}=\frac{\beta^{2}}{\lambda_{1}^{5}} \theta_{1} a_{1}^{5} \quad e \quad a_{1}^{2} b_{1}=\frac{\beta}{\lambda_{1}^{3}} \theta_{2} a_{1}^{5}, \quad \text { com } \quad \lambda_{1}, \beta \neq 0 .
$$

Prova: Seja $\mathcal{B}$ uma nilálgebra como no enunciado do lema e $\left(a_{1}, b_{1}\right)$ um elemento de $\mathcal{P}(\mathcal{B})$. Então $a_{1}$ e $b_{1}$ são da forma $a_{1}=\lambda b+\lambda_{1} a+\lambda_{2} a^{2}+\lambda_{3} a^{3}+$ $\lambda_{4} a^{4}+\lambda_{5} a^{5}$ e $b_{1}=\beta b+\beta_{1} a+\beta_{2} a^{2}+\beta_{3} a^{3}+\beta_{4} a^{4}+\beta_{5} a^{5}, \mathrm{e}$

$$
\begin{aligned}
a_{1}^{2}= & \lambda^{2} b^{2}+2 \lambda_{2} \lambda a^{2} b+\lambda_{1}^{2} a^{2}+2 \lambda_{1} \lambda_{2} a^{3}+2 \lambda_{1} \lambda_{3} a^{4}+2 \lambda_{1} \lambda_{4} a^{5}+\lambda_{2}^{2} a^{4} \\
& +2 \lambda_{2} \lambda_{3} a^{5} \\
= & \lambda_{1}^{2} a^{2}+2 \lambda_{1} \lambda_{2} a^{3}+\left(2 \lambda_{1} \lambda_{3}+\lambda_{2}^{2}\right) a^{4}+\left(\lambda^{2} \theta_{1}+2 \lambda_{2} \lambda \theta_{2}+2 \lambda_{1} \lambda_{4}+2 \lambda_{2} \lambda_{3}\right) a^{5}, \\
a_{1}^{3}= & \lambda_{1}^{2} \lambda a^{2} b+\lambda_{1}^{3} a^{3}+2 \lambda_{1}^{2} \lambda_{2} a^{4}+\lambda_{1}\left(2 \lambda_{1} \lambda_{3}+\lambda_{2}^{2}\right) a^{5}+\lambda_{1}^{2} \lambda_{2} a^{4}+2 \lambda_{1} \lambda_{2}^{2} a^{5} \\
& +\lambda_{1}^{2} \lambda_{3} a^{5} \\
= & \lambda_{1}^{3} a^{3}+3 \lambda_{1}^{2} \lambda_{2} a^{4}+\lambda_{1}\left(\lambda \lambda_{1} \theta_{2}+3 \lambda_{1} \lambda_{3}+3 \lambda_{2}^{2}\right) a^{5}, \\
a_{1}^{4}= & \lambda_{1}^{4} a^{4}+3 \lambda_{1}^{3} \lambda_{2} a^{5}+\lambda_{2} \lambda_{1}^{3} a^{5}=\lambda_{1}^{4} a^{4}+4 \lambda_{1}^{3} \lambda_{2} a^{5}, \\
a_{1}^{5}= & \lambda_{1}^{5} a^{5} \neq 0,
\end{aligned}
$$


do que segue que $\lambda_{1} \neq 0$. Dado que,

$$
\begin{aligned}
a_{1} b_{1}= & \lambda \beta b^{2}+\lambda \beta_{2} a^{2} b+\lambda_{1} \beta_{1} a^{2}+\lambda_{1} \beta_{2} a^{3}+\lambda_{1} \beta_{3} a^{4}+\lambda_{1} \beta_{4} a^{5}+\lambda_{2} \beta a^{2} b+ \\
& \lambda_{2} \beta_{1} a^{3}+\lambda_{2} \beta_{2} a^{4}+\lambda_{2} \beta_{3} a^{5}+\lambda_{3} \beta_{1} a^{4}+\lambda_{3} \beta_{2} a^{5}+\lambda_{4} \beta_{1} a^{5} \\
= & \lambda_{1} \beta_{1} a^{2}+\left(\lambda_{1} \beta_{2}+\lambda_{2} \beta_{1}\right) a^{3}+\left(\lambda_{1} \beta_{3}+\lambda_{2} \beta_{2}+\lambda_{3} \beta_{1}\right) a^{4}+\left(\lambda \beta \theta_{1}+\lambda \beta_{2} \theta_{2}\right. \\
& \left.+\lambda_{1} \beta_{4}+\lambda_{2} \beta \theta_{2}+\lambda_{3} \beta_{2}+\lambda_{4} \beta_{1}+\lambda_{2} \beta_{3}\right) a^{5} \\
= & 0,
\end{aligned}
$$

temos que $\lambda_{1} \beta_{1}=0$ e como $\lambda_{1} \neq 0$, segue que $\beta_{1}=0$. Igualmente $\beta_{2}=$ $\beta_{3}=0$ e portanto $\beta_{4}=-\frac{\lambda \beta \theta_{1}+\lambda_{2} \beta \theta_{2}}{\lambda_{1}}$. Assim

$$
b_{1}=\beta b-\frac{\beta\left(\lambda \theta_{1}+\lambda_{2} \theta_{2}\right)}{\lambda_{1}} a^{4}+\beta_{5} a^{5}, \quad \text { com } \quad \beta \neq 0 .
$$

Logo

$$
b_{1}^{2}=\beta^{2} b^{2}=\beta^{2} \theta_{1} a^{5}=\frac{\beta^{2}}{\lambda_{1}^{5}} \theta_{1} a_{1}^{5} \quad \text { e } \quad a_{1}^{2} b_{1}=\beta \lambda_{1}^{2} a^{2} b=\beta \lambda_{1}^{2} \theta_{2} a^{5}=\frac{\beta}{\lambda_{1}^{3}} \theta_{2} a_{1}^{5} .
$$

Se $\theta_{1}=0$ e $\theta_{2}=1$, no lema anterior, temos a álgebra $\mathcal{B}_{7}$, portanto se $\left(a_{1}, b_{1}\right)$ é um elemento de $\mathcal{P}\left(\mathcal{B}_{7}\right)$, então

$$
b_{1}^{2}=0 \quad \text { e } \quad a_{1}^{2} b_{1}=\frac{\beta}{\lambda_{1}^{3}} a_{1}^{5}, \quad \text { com } \quad \lambda_{1}, \beta \neq 0,
$$

com o qual fica claro que

$$
\mathcal{B}_{7} \nsucceq \mathcal{B}_{6} \quad \text { e } \quad \mathcal{B}_{7} \nsubseteq \mathcal{B}_{8}
$$

Quando $\theta_{1}=1$ e $\theta_{2}=0$, temos a álgebra $\mathcal{B}_{8}$, logo para cada par $\left(a_{1}, b_{1}\right)$ em $\mathcal{P}\left(\mathcal{B}_{8}\right)$, segue que

$$
b_{1}^{2}=\frac{\beta^{2}}{\lambda_{1}^{5}} a_{1}^{5} \quad \text { e } \quad a_{1}^{2} b_{1}=0, \quad \text { com } \quad \lambda_{1}, \beta \neq 0,
$$

portanto

$$
\mathcal{B}_{6} \nsucceq \mathcal{B}_{8}
$$


Assim

$$
\mathcal{B}_{6} ¥ \mathcal{B}_{i}, \quad \mathcal{B}_{7} \not \mathcal{B}_{j} \quad \text { e } \quad \mathcal{B}_{8} \not \mathcal{B}_{k}, \quad \text { para } i \neq 6, j \neq 7 \text { e } k \neq 8 \text {. }
$$

Para concluir o análise das condições de isomorfismo das álgebras da lista anterior, resta estudar as álgebras do conjunto $C 1$. Faremos isto a continuação.

Lema 4.21 Seja $\alpha$ diferente de -3 em $F^{*}$. Então

$$
\mathcal{B}_{3}(\alpha) \cong \mathcal{B}_{2}(\alpha)
$$

Prova: Seja $\alpha$ diferente de 0 e $-3,(a, b)$ em $\mathcal{P}\left(\mathcal{B}_{3}(\alpha)\right)$ e os produtos respeito a base determinada por $(a, b)$ dados por $b^{2}=\alpha a^{4}, a^{2} b=a^{4}$ e $a^{3} b=-a^{5}$. Definimos os seguinte elementos $a_{1}:=\frac{7}{4 \alpha(3+\alpha)} b+a-\frac{1}{4(3+\alpha)} a^{2} \quad$ e $\quad b_{1}:=b-\frac{3}{2 \alpha(3+\alpha)} a^{3}-\frac{3(7+\alpha)}{8 \alpha(3+\alpha)} a^{4}$.

Então

$$
\begin{aligned}
a_{1} b_{1}= & -\frac{7}{4 \alpha(3+\alpha)} b^{2}-\frac{1}{4(3+\alpha)} b a^{2}-\frac{21}{8 \alpha(3+\alpha)^{2}} b a^{3}-\frac{3}{2(3+\alpha)} a^{4} \\
& +\frac{3}{8(3+\alpha)^{2}} a^{5}-\frac{3(7+\alpha)}{8 \alpha(3+\alpha)^{2}} a^{5} \\
= & \frac{1}{2(3+\alpha)}\left[\frac{7}{2}-\frac{1}{2}-3\right] a^{4}+\frac{1}{8 \alpha(3+\alpha)}[21+3 \alpha-21-3 \alpha] a^{5} \\
= & 0
\end{aligned}
$$

$\mathrm{e}$

$$
\begin{aligned}
a_{1}^{2} & =\frac{49}{16 \alpha^{2}(3+\alpha)^{2}} b^{2}-\frac{7}{8 \alpha(3+\alpha)^{2}} b a^{2}+a^{2}-\frac{1}{2(3+\alpha)} a^{3}+\frac{1}{16(3+\alpha)^{2}} a^{4} \\
& =a^{2}-\frac{1}{2(3+\alpha)} a^{3}+\frac{33+\alpha}{16 \alpha(3+\alpha)^{2}} a^{4}
\end{aligned}
$$




$$
\begin{aligned}
a_{1}^{3}= & \frac{7}{4 \alpha(3+\alpha)} a^{2} b-\frac{7}{8 \alpha(3+\alpha)^{2}} b a^{3}+a^{3}-\frac{1}{2(3+\alpha)} a^{4}+\frac{35+\alpha}{16 \alpha(3+\alpha)^{2}} a^{5} \\
& -\frac{1}{4(3+\alpha)} a^{4}+\frac{1}{8(3+\alpha)^{2}} a^{5}, \\
= & a^{3}+\frac{7-3 \alpha}{4 \alpha(3+\alpha)} a^{4}+\frac{49+3 \alpha}{16 \alpha(3+\alpha)^{2}} a^{5}, \\
a_{1}^{4}= & \frac{7}{4 \alpha(3+\alpha)} a^{3} b+a^{4}-\frac{1}{4(3+\alpha)} a^{5}+\frac{7-3 \alpha}{4 \alpha(3+\alpha)} a^{5} \\
= & a^{4}-\frac{1}{(3+\alpha)} a^{5}, \\
a_{1}^{5}= & a^{5} .
\end{aligned}
$$

Isto mostra que $a_{1} b_{1}=0$ e $a_{1}$ tem nilíndice máximo, e sendo claro que $b_{1}$ não pertence a $A_{a_{1}}$ segue que $\left(a_{1}, b_{1}\right)$ está em $\mathcal{P}\left(\mathcal{B}_{5}\right)$.

Agora

$$
\begin{aligned}
b_{1}^{2} & =b^{2}-\frac{3}{(3+\alpha)} a^{3} b=\alpha a^{4}+\frac{3}{(3+\alpha)} a^{5}=\alpha\left[a_{1}^{4}+\frac{1}{(3+\alpha)} a_{1}^{5}\right]+\frac{3}{(3+\alpha)} a_{1}^{5} \\
& =\alpha a_{1}^{4}+a_{1}^{5}
\end{aligned}
$$

e

$$
a_{1}^{2} b_{1}=a^{4}-\frac{1}{(3+\alpha)} a^{5}=\left[a_{1}^{4}+\frac{1}{(3+\alpha)} a_{1}^{5}\right]-\frac{1}{(3+\alpha)} a_{1}^{5}=a_{1}^{4},
$$

o que mostra o isomorfismo.

Assim neste caso resta ver como são as álgebras $\mathcal{B}_{2}(\alpha)$ e $\mathcal{B}_{3}(\alpha)$, para $\alpha$ igual a 0 e -3 . Denotaremos estas álgebras assim:

$$
\begin{aligned}
& \mathcal{B}_{3}(0): \quad a^{2} b=a^{4}, \quad a^{3} b=-a^{5} ; \\
& \mathcal{B}_{3}(-3): \quad b^{2}=-3 a^{4}, \quad a^{2} b=a^{4}, \quad a^{3} b=-a^{5} ; \\
& \mathcal{B}_{2}(-3): \quad b^{2}=-3 a^{4}+a^{5}, \quad a^{2} b=a^{4}, \quad a^{3} b=-a^{5} ; \\
& \mathcal{B}_{2}(0): \quad b^{2}=a^{5}, \quad a^{2} b=a^{4}, \quad a^{3} b=-a^{5} .
\end{aligned}
$$


Demonstraremos que estas álgebras não são isomorfas, da mesma maneira que fizemos para as álgebras do conjunto $C 3$.

Lema 4.22 Seja $\mathcal{B}$ uma nilálgebra comutativa de p.a. de dimensão e nilíndice 6, com base $\left\{b, a, a^{2}, a^{3}, a^{4}, a^{5}\right\}$ tal que $b^{2}=\theta_{1} a^{4}+\theta_{2} a^{5}, a^{2} b=a^{4}$ e $a^{3} b=$ $-a^{5}$ e os outros produtos não triviais são zero. Então para cada par $\left(a_{1}, b_{1}\right)$ em $\mathcal{P}(\mathcal{B})$, existem $\lambda_{1}, \beta \in F^{*}$ e $\lambda_{2}, \lambda \in F$, tais que

$$
b_{1}^{2}=\frac{\beta^{2}}{\lambda_{1}^{4}} \theta_{1} a_{1}^{4}+\frac{\beta^{2}}{\lambda_{1}^{6}}\left(-4 \lambda_{2} \theta_{1}+\theta_{2} \lambda_{1}+2 \lambda \theta_{1}+2 \lambda_{2}\right) a_{1}^{5}
$$

$e$

$$
a_{1}^{2} b_{1}=\frac{\beta}{\lambda_{1}^{2}} a_{1}^{4}+\frac{\beta}{\lambda_{1}^{4}}\left(7 \lambda_{2}+\lambda \theta_{1}\right) a_{1}^{5}
$$

Prova: Seja $\mathcal{B}$ como no enunciado do lema e $\left(a_{1}, b_{1}\right) \in \mathcal{P}(\mathcal{B})$. Então $a_{1}$ e $b_{1}$ exprimem-se de maneira única na forma, $a_{1}=\lambda b+\lambda_{1} a+\lambda_{2} a^{2}+\lambda_{3} a^{3}+$ $\lambda_{4} a^{4}+\lambda_{5} a^{5}$ e $b_{1}=\beta b+\beta_{1} a+\beta_{2} a^{2}+\beta_{3} a^{3}+\beta_{4} a^{4}+\beta_{5} a^{5}$. Antes de calcular os produtos $b_{1}^{2}$ e $a_{1}^{2} b_{1}$, vejamos como são as potências de $a_{1}$.

$$
\begin{aligned}
a_{1}^{2}= & \lambda^{2} b^{2}+2 \lambda_{2} \lambda a^{2} b+2 \lambda_{3} \lambda a^{3} b+\lambda_{1}^{2} a^{2}+2 \lambda_{1} \lambda_{2} a^{3}+2 \lambda_{1} \lambda_{3} a^{4}+2 \lambda_{1} \lambda_{4} a^{5} \\
& +\lambda_{2}^{2} a^{4}+2 \lambda_{2} \lambda_{3} a^{5} \\
= & \lambda_{1}^{2} a^{2}+2 \lambda_{1} \lambda_{2} a^{3}+\left(\lambda^{2} \theta_{1}+2 \lambda \lambda_{2}+2 \lambda_{1} \lambda_{3}+\lambda_{2}^{2}\right) a^{4}+\left(\lambda^{2} \theta_{2}-2 \lambda \lambda_{3}\right. \\
& \left.+2 \lambda_{1} \lambda_{4}+2 \lambda_{2} \lambda_{3}\right) a^{5} \\
a_{1}^{3}= & \lambda_{1}^{2} \lambda a^{2} b+2 \lambda_{1} \lambda_{2} \lambda a^{3} b+\lambda_{1}^{3} a^{3}+2 \lambda_{1}^{2} \lambda_{2} a^{4}+\lambda_{1}\left(\lambda^{2} \theta_{1}+2 \lambda \lambda_{2}+2 \lambda_{1} \lambda_{3}\right. \\
& \left.+\lambda_{2}^{2}\right) a^{5}+\lambda_{1}^{2} \lambda_{2} a^{4}+2 \lambda_{1} \lambda_{2}^{2} a^{5}+\lambda_{1}^{2} \lambda_{3} a^{5} \\
= & \lambda_{1}^{3} a^{3}+\left(\lambda \lambda_{1}^{2}+3 \lambda_{1}^{2} \lambda_{2}\right) a^{4}+\lambda_{1}\left(\lambda^{2} \theta_{1}+3 \lambda_{1} \lambda_{3}+3 \lambda_{2}^{2}\right) a^{5}, \\
a_{1}^{4}= & \lambda \lambda_{1}^{3} a^{3} b+\lambda_{1}^{4} a^{4}+\left(\lambda \lambda_{1}^{3}+3 \lambda_{1}^{3} \lambda_{2}\right) a^{5}+\lambda_{1}^{3} \lambda_{2} a^{5}=\lambda_{1}^{4} a^{4}+4 \lambda_{1}^{3} \lambda_{2} a^{5}, \\
a_{1}^{5}= & \lambda_{1}^{5} a^{5} .
\end{aligned}
$$

Como $\lambda_{1} \neq 0$, pois $a_{1}$ tem nilíndice máximo, temos que

$$
a^{5}=\frac{1}{\lambda_{1}^{5}} a_{1}^{5} \quad \text { e } \quad a^{4}=\frac{1}{\lambda_{1}^{4}} a_{1}^{4}-4 \frac{\lambda_{2}}{\lambda_{1}^{6}} a_{1}^{5} .
$$


Agora,

$$
\begin{aligned}
a_{1} b_{1}= & \lambda \beta b^{2}+\lambda \beta_{2} a^{2} b+\lambda \beta_{3} a^{3} b+\lambda_{1} \beta_{1} a^{2}+\lambda_{1} \beta_{2} a^{3}+\lambda_{1} \beta_{3} a^{4}+\lambda_{1} \beta_{4} a^{5} \\
& +\lambda_{2} \beta a^{2} b+\lambda_{2} \beta_{1} a^{3}+\lambda_{2} \beta_{2} a^{4}+\lambda_{2} \beta_{3} a^{5}+\lambda_{3} \beta a^{3} b+\lambda_{3} \beta_{1} a^{4}+ \\
& \lambda_{3} \beta_{2} a^{5}+\lambda_{4} \beta_{1} a^{5} \\
= & \lambda_{1} \beta_{1} a^{2}+\left(\lambda_{1} \beta_{2}+\lambda_{2} \beta_{1}\right) a^{3}+\left(\lambda \beta \theta_{1}+\lambda \beta_{2}+\lambda_{1} \beta_{3}+\lambda_{2} \beta+\lambda_{2} \beta_{2}\right. \\
& \left.+\lambda_{3} \beta_{1}\right) a^{4}+\left(\lambda \beta \theta_{1}-\lambda \beta_{3}+\lambda_{1} \beta_{4}+\lambda_{2} \beta_{3}-\lambda_{3} \beta+\lambda_{3} \beta_{2}+\lambda_{4} \beta_{1}\right) a^{5} \\
= & 0,
\end{aligned}
$$

$\operatorname{logo} \lambda_{1} \beta_{1}=0$ e como $\lambda_{1} \neq 0$, segue que $\beta_{1}=0$. Igualmente $\beta_{2}=0$, e portanto $\beta_{3}=-\frac{\lambda \beta \theta_{1}+\lambda_{2} \beta}{\lambda_{1}}$ e

$$
\beta_{4}=-\frac{\beta\left(\lambda \lambda_{1} \theta_{2}+\lambda^{2} \theta_{1}+\lambda \lambda_{2}-\lambda_{3} \lambda_{1}-\lambda_{2} \lambda \theta_{1}-\lambda_{2}^{2}\right)}{\lambda_{1}^{2}} .
$$

Assim

$b_{1}=\beta b-\frac{\beta\left(\lambda \theta_{1}+\lambda_{2}\right)}{\lambda_{1}} a^{3}-\frac{\beta\left(\lambda \lambda_{1} \theta_{2}+\lambda^{2} \theta_{1}+\lambda \lambda_{2}-\lambda_{3} \lambda_{1}-\lambda_{2} \lambda \theta_{1}-\lambda_{2}^{2}\right)}{\lambda_{1}^{2}} a^{4}+\beta_{5} a^{5}$, $\operatorname{com} \beta \neq 0$. Portanto

$$
\begin{aligned}
b_{1}^{2} & =\beta^{2} b^{2}-\frac{2 \beta^{2}\left(\lambda \alpha+\lambda_{2}\right)}{\lambda_{1}} a^{3} b=\beta^{2} \alpha a^{4}+\beta^{2} \theta_{2} a^{5}+\frac{2 \beta^{2}\left(\lambda \theta_{1}+\lambda_{2}\right)}{\lambda_{1}} a^{5} \\
& =\beta^{2} \theta_{1} a^{4}+\frac{\beta^{2}\left(\theta_{2} \lambda_{1}+2 \lambda \theta_{1}+2 \lambda_{2}\right)}{\lambda_{1}} a^{5} \\
& =\beta^{2} \theta_{1}\left[\frac{1}{\lambda_{1}^{4}} a_{1}^{4}-4 \frac{\lambda_{2}}{\lambda_{1}^{6}} a_{1}^{5}\right]+\frac{\beta^{2}\left(\theta_{2} \lambda_{1}+2 \lambda \theta_{1}+2 \lambda_{2}\right)}{\lambda_{1}}\left[\frac{1}{\lambda_{1}^{5}} a_{1}^{5}\right] \\
& =\frac{\beta^{2}}{\lambda_{1}^{4}} \theta_{1} a_{1}^{4}+\frac{\beta^{2}}{\lambda_{1}^{6}}\left(-4 \lambda_{2} \theta_{1}+\theta_{2} \lambda_{1}+2 \lambda \theta_{1}+2 \lambda_{2}\right) a_{1}^{5}, \\
a_{1}^{2} b_{1} & =\beta \lambda_{1}^{2} a^{2} b-\frac{\lambda_{1}^{2} \beta\left(\lambda \theta_{1}+\lambda_{2}\right)}{\lambda_{1}} a^{5}+2 \lambda_{1} \lambda_{2} \beta a^{3} b=\beta \lambda_{1}^{2} a^{4}-\beta \lambda_{1}\left(\lambda \theta_{1}+3 \lambda_{2}\right) a^{5} \\
& =\beta \lambda_{1}^{2}\left[\frac{1}{\lambda_{1}^{4}} a_{1}^{4}-4 \frac{\lambda_{2}}{\lambda_{1}^{6}} a_{1}^{5}\right]-\beta \lambda_{1}\left(\lambda \theta_{1}+3 \lambda_{2}\right)\left[\frac{1}{\lambda_{1}^{5}} a_{1}^{5}\right] \\
& =\frac{\beta}{\lambda_{1}^{2}} a_{1}^{4}+\frac{\beta}{\lambda_{1}^{4}}\left(7 \lambda_{2}+\lambda \theta_{1}\right) a_{1}^{5},
\end{aligned}
$$


o que demonstra o lema.

Para $\mathcal{B}_{3}(0)$, temos que $\theta_{1}=\theta_{2}=0$ no lema anterior, portanto para cada par $\left(a_{1}, b_{1}\right)$ em $\mathcal{P}\left(\mathcal{B}_{3}(0)\right)$,

$$
b_{1}^{2}=\frac{2 \beta^{2} \lambda_{2}}{\lambda_{1}^{6}} a_{1}^{5} \quad \text { e } \quad a_{1}^{2} b_{1}=\frac{\beta}{\lambda_{1}^{2}} a_{1}^{4}-\frac{7 \beta \lambda_{2}}{\lambda_{1}^{4}} a_{1}^{5} \quad \text { com } \quad \lambda_{1}, \beta \in F^{*} \quad \text { e } \quad \lambda_{2} \in F .
$$

Do anterior é claro que $\mathcal{B}_{3}(0)$ não pode ser isomorfa a nenhuma das outras álgebras da ultima lista, pois se fosse isomorfa a qualquer um delas então $-\frac{7 \beta \lambda_{2}}{\lambda_{1}^{4}}=0$, e como $\lambda_{1}$ e $\beta$ são não nulos, $\lambda_{2}=0$ e portanto $b_{1}^{2}=0$.

Para $\mathcal{B}_{3}(-3)$ temos que temos que $\theta_{1}=-3$ e $\theta_{2}=0$, logo se $\left(a_{1}, b_{1}\right)$ pertence a $\mathcal{P}\left(\mathcal{B}_{3}(-3)\right)$, então

$$
b_{1}^{2}=\frac{-3 \beta^{2}}{\lambda_{1}^{4}} a_{1}^{4}+\frac{2 \beta^{2}\left(7 \lambda_{2}-3 \lambda\right)}{\lambda_{1}^{6}} a_{1}^{5} \quad \text { e } \quad a_{1}^{2} b_{1}=\frac{\beta}{\lambda_{1}^{2}} a_{1}^{4}+\frac{\beta\left(7 \lambda_{2}-3 \lambda\right)}{\lambda_{1}^{4}} a_{1}^{5},
$$

com $\lambda_{1}, \beta \in F^{*}$ e $\lambda_{2}, \lambda \in F$. Portanto para $\mathcal{B}_{3}(-3)$ ser isomorfa a alguma das outras duas álgebras teria que se cumprir que $-\frac{\beta\left(7 \lambda_{2}-3 \lambda\right)}{\lambda_{1}^{4}}=0, \operatorname{logo} 7 \lambda_{2}=3 \lambda$ e assim $b_{1}^{2}=\frac{-3 \beta^{2}}{\lambda_{1}^{4}} a_{1}^{4}$, isto é, o escalar correspondente a $a_{1}^{5}$ é zero.

Por ultimo, $\theta_{1}=0$ e $\theta_{2}=1$, para $\mathcal{B}_{2}(0)$. Logo para cada par $\left(a_{1}, b_{1}\right)$ em $\mathcal{P}\left(\mathcal{B}_{2}(0)\right)$, segue que

$$
b_{1}^{2}=\frac{\beta^{2}\left(\lambda_{1}+2 \lambda_{2}\right)}{\lambda_{1}^{6}} a_{1}^{5},
$$

isto é, o escalar correspondente a $a_{1}^{4}$ é zero o que mostra que $\mathcal{B}_{2}(0)$ não pode ser isomorfa a $\mathcal{B}_{2}(-3)$. Do analise anterior concluímos que

$$
\mathcal{B}_{3}(0) \nsucceq \mathcal{B}_{3}(-3) \not \mathcal{B}_{2}(-3) \not \mathcal{B}_{2}(0) \text {. }
$$

Do lema anterior desprende-se também o seguinte corolário.

Corolário 4.23 Seja $\alpha$ diferente de -3 e de 0 e $\alpha^{\prime}$ em F. Então

$$
\mathcal{B}_{3}(\alpha) \cong \mathcal{B}_{3}\left(\alpha^{\prime}\right)
$$

se, e somente se, $\alpha^{\prime}=\alpha$. 
Prova: Seja $\alpha$ diferente de -3 em $F^{*}$ e os produtos respeito a base determinada pelo par $(a, b) \in \mathcal{P}\left(\mathcal{B}_{3}(\alpha)\right)$, dados por $b^{2}=\alpha a^{4}, a^{2} b=a^{4}$ e $a^{3} b=-a^{5}$. Se $\left(a_{1}, b_{1}\right)$ está em $\mathcal{P}\left(\mathcal{B}_{3}(\alpha)\right)$, então pelo lema anterior

$$
b_{1}^{2}=\frac{\beta^{2}}{\lambda_{1}^{4}} \alpha a_{1}^{4}+\frac{\beta^{2}}{\lambda_{1}^{6}}\left(-4 \lambda_{2} \alpha+2 \lambda \alpha+2 \lambda_{2}\right) a_{1}^{5}
$$

e

$$
a_{1}^{2} b_{1}=\frac{\beta}{\lambda_{1}^{2}} a_{1}^{4}+\frac{\beta}{\lambda_{1}^{4}}\left(7 \lambda_{2}+\lambda \alpha\right) a_{1}^{5}, \quad \text { com, } \quad \lambda_{1}, \beta \neq 0 .
$$

Logo para $\alpha^{\prime}$ em $F, \mathcal{B}_{3}(\alpha) \cong \mathcal{B}_{3}\left(\alpha^{\prime}\right)$ se, e somente se,

$$
\alpha^{\prime}=\frac{\beta^{2}}{\lambda_{1}^{4}} \alpha, \quad \frac{\beta^{2}}{\lambda_{1}^{6}}\left(-4 \lambda_{2} \alpha+2 \lambda \alpha+2 \lambda_{2}\right)=0, \quad \frac{\beta}{\lambda_{1}^{2}}=1 \quad \text { e } \frac{\beta}{\lambda_{1}^{4}}\left(7 \lambda_{2}+\lambda \alpha\right)=0,
$$

portanto $\beta=\lambda_{1}^{2}$ e assim $\alpha^{\prime}=\alpha$.

Com isto concluímos o estudo das condições de isomorfismo das álgebras da lista inicial. Resta então analisar como são as classes dadas por $\mathcal{B}_{1}(\alpha, \theta, \gamma)$ e $\mathcal{B}_{5}(\alpha)$.

Lema 4.24 Sejam $\alpha$ e $\alpha^{\prime}$ em $F^{*}$. Então

$$
\mathcal{B}_{5}(\alpha) \cong \mathcal{B}_{5}\left(\alpha^{\prime}\right)
$$

se, e somente se, existir um $\rho$ em $F^{*}$ tal que $\alpha^{\prime}=\rho^{2} \alpha$.

Prova: Sejam $\alpha$ em $F$ e $(a, b)$ em $\mathcal{P}\left(\mathcal{B}_{5}(\alpha)\right)$ tal que $b^{2}=\alpha a^{4}$ e os outros produtos não triviais são zero. Se $\left(a_{1}, b_{1}\right)$ está em $\mathcal{P}\left(\mathcal{B}_{5}(\alpha)\right)$, então $a_{1}=$ $\lambda b+\lambda_{1} a+\lambda_{2} a^{2}+\lambda_{3} a^{3}+\lambda_{4} a^{4}+\lambda_{5} a^{5}$ e $b_{1}=\beta b+\beta_{1} a+\beta_{2} a^{2}+\beta_{3} a^{3}+\beta_{4} a^{4}+\beta_{5} a^{5}$, e uma vez que $a_{1}$ tem nilíndice máximo, temos que

$$
\begin{aligned}
a_{1}^{2} & =\lambda^{2} b^{2}+\lambda_{1}^{2} a^{2}+2 \lambda_{1} \lambda_{2} a^{3}+2 \lambda_{1} \lambda_{3} a^{4}+2 \lambda_{1} \lambda_{4} a^{5}+\lambda_{2}^{2} a^{4}+2 \lambda_{2} \lambda_{3} a^{5} \\
& =\lambda_{1}^{2} a^{2}+2 \lambda_{1} \lambda_{2} a^{3}+\left(2 \lambda_{1} \lambda_{3}+\lambda_{2}^{2}+\lambda^{2} \alpha\right) a^{4}+\left(2 \lambda_{1} \lambda_{4}+2 \lambda_{2} \lambda_{3}\right) a^{5}, \\
a_{1}^{3} & =\lambda_{1}^{3} a^{3}+2 \lambda_{1}^{2} \lambda_{2} a^{4}+\lambda_{1}\left(2 \lambda_{1} \lambda_{3}+\lambda_{2}^{2}+\lambda^{2} \alpha\right) a^{5}+\lambda_{1}^{2} \lambda_{2} a^{4}+2 \lambda_{1} \lambda_{2}^{2} a^{5}+\lambda_{1}^{2} \lambda_{3} a^{5} \\
& =\lambda_{1}^{3} a^{3}+3 \lambda_{1}^{2} \lambda_{2} a^{4}+\lambda_{1}\left(3 \lambda_{1} \lambda_{3}+3 \lambda_{2}^{2}+\lambda^{2} \alpha\right) a^{5}, \\
a_{1}^{4} & =\lambda_{1}^{4} a^{4}+3 \lambda_{1}^{3} \lambda_{2} a^{5}+\lambda_{2} \lambda_{1}^{3} a^{5}=\lambda_{1}^{4} a^{4}+4 \lambda_{1}^{3} \lambda_{2} a^{5}, \\
a_{1}^{5} & =\lambda_{1}^{5} a^{5} \neq 0,
\end{aligned}
$$


portanto $\lambda_{1} \neq 0$. É fácil ver que $a^{5}=\frac{1}{\lambda_{1}^{5}} a_{1}^{5}$ e $a^{4}=\frac{1}{\lambda_{1}^{4}} a_{1}^{4}-4 \frac{\lambda_{2}}{\lambda_{1}^{6}} a_{1}^{5}$. Agora,

$$
\begin{aligned}
a_{1} b_{1}= & \lambda \beta b^{2}+\lambda_{1} \beta_{1} a^{2}+\lambda_{1} \beta_{2} a^{3}+\lambda_{1} \beta_{3} a^{4}+\lambda_{1} \beta_{4} a^{5}+\lambda_{2} \beta_{1} a^{3}+\lambda_{2} \beta_{2} a^{4}+ \\
& \lambda_{2} \beta_{3} a^{5}+\lambda_{3} \beta_{1} a^{4}+\lambda_{3} \beta_{2} a^{5}+\lambda_{4} \beta_{1} a^{5} \\
= & \lambda_{1} \beta_{1} a^{2}+\left(\lambda_{1} \beta_{2}+\lambda_{2} \beta_{1}\right) a^{3}+\left(\lambda \beta \alpha+\lambda_{1} \beta_{3}+\lambda_{2} \beta_{2}+\lambda_{3} \beta_{1}\right) a^{4}+\left(\lambda_{1} \beta_{4}+\right. \\
& \left.\lambda_{2} \beta_{3}+\lambda_{3} \beta_{2}+\lambda_{4} \beta_{1}\right) a^{5} \\
= & 0
\end{aligned}
$$

$\operatorname{logo} \lambda_{1} \beta_{1}=0$ e como $\lambda_{1} \neq 0$, segue que $\beta_{1}=0$, igualmente $\beta_{2}=0$ e portanto $\beta_{3}=\frac{-\lambda \beta \alpha}{\lambda_{1}}$ e $\beta_{4}=\frac{\lambda_{2} \lambda \beta \alpha}{\lambda_{1}^{2}}$. Assim

$$
b_{1}=\beta b-\frac{\beta \lambda \alpha}{\lambda_{1}} a^{3}+\frac{\lambda_{2} \lambda \beta \alpha}{\lambda_{1}^{2}} a^{4}+\beta_{5} a^{5}, \quad \text { com } \quad \beta \neq 0 .
$$

$\log O$

$$
b_{1}^{2}=\beta^{2} b^{2}=\beta^{2} \alpha a^{4}=\frac{\beta^{2}}{\lambda_{1}^{4}} \alpha a_{1}^{4}-4 \frac{\beta^{2} \lambda_{2}}{\lambda_{1}^{6}} \alpha a_{1}^{5}
$$

$\mathrm{e}$

$$
a_{1}^{2} b_{1}=\lambda_{1}^{2} a^{2} b=-\frac{\beta \lambda \lambda_{1}^{2}}{\lambda_{1}} \alpha a^{5}=-\beta \lambda \lambda_{1} \alpha \frac{1}{\lambda_{1}^{5}} a_{1}^{5}=-\frac{\beta \lambda}{\lambda_{1}^{4}} \alpha a_{1}^{5} .
$$

Portanto dado um $\alpha^{\prime}$ em $F^{*}$, temos que $\mathcal{B}_{5}(\alpha) \cong \mathcal{B}_{5}\left(\alpha^{\prime}\right)$, se e somente se,

$$
\alpha^{\prime}=\frac{\beta^{2}}{\lambda_{1}^{4}} \alpha, \quad-\frac{4 \beta^{2} \lambda_{2}}{\lambda_{1}^{6}} \alpha=0 \quad \text { e } \quad-\frac{\beta \lambda}{\lambda_{1}^{4}} \alpha=0 .
$$

Como $\beta, \lambda_{1}$ e $\alpha$ são não nulos temos que $\lambda=\lambda_{2}=0$ e assim $\alpha^{\prime}=\rho^{2} \alpha$, com $\rho$ em $F^{*}$.

Lema 4.25 Para todo $\alpha, \theta$ e $\gamma$ em $F$,

$$
\mathcal{B}_{1}(\alpha, \theta, \gamma) \cong \mathcal{B}_{1}\left(\alpha^{\prime}, \theta^{\prime}, \gamma^{\prime}\right)
$$

se, e somente se, existir $\rho_{1}, \rho_{2} \in F^{*}$, tal que

$$
\alpha^{\prime}=\frac{\alpha}{\rho_{1}^{2}}, \quad \theta^{\prime}=\frac{\theta}{\rho_{2}^{3}} \quad \text { e } \quad \gamma^{\prime}=\frac{1}{\rho_{2}^{2}}\left(\gamma+\frac{\rho_{1}+\rho_{2}}{\rho_{1}-\rho_{2}} \alpha^{\prime}\right) .
$$


Prova: $\quad \operatorname{Sejam~} \alpha, \theta, \gamma$ em $F, \mathcal{B}$ a álgebra $\mathcal{B}_{1}(\alpha, \theta, \gamma)$ e $(a, b) \in \mathcal{P}(B)$, tal que $b^{2}=-a^{2}+\alpha a_{4}+\theta a^{5}, a^{2} b=a^{3}+\gamma a^{5}, a^{3} b=-a^{4}$ e $a^{4} b=a^{5}$. Todo par $\left(a_{1}, b_{1}\right) \in \mathcal{P}(\mathcal{B})$, exprime-se de maneira única como $a_{1}=\lambda b+\lambda_{1} a+\lambda_{2} a^{2}+$ $\lambda_{3} a^{3}+\lambda_{4} a^{4}+\lambda_{5} a^{5}$ e $b_{1}=\beta b+\beta_{1} a+\beta_{2} a^{2}+\beta_{3} a^{3}+\beta_{4} a^{4}+\beta_{5} a^{5}$. Calculando as potências de $a_{1}$, obtemos que

$$
\begin{aligned}
a_{1}^{2}= & \lambda^{2} b^{2}+2 \lambda \lambda_{2} a^{2} b+2 \lambda \lambda_{3} a^{3} b+2 \lambda \lambda_{4} a^{4} b+\lambda_{1}^{2} a^{2}+2 \lambda_{1} \lambda_{2} a^{3}+2 \lambda_{1} \lambda_{3} a^{4}+ \\
& 2 \lambda_{1} \lambda_{4} a^{5}+\lambda_{2}^{2} a^{4}+2 \lambda_{2} \lambda_{3} a^{5} \\
= & \left(\lambda_{1}^{2}-\lambda^{2}\right) a^{2}+2 \lambda_{2}\left(\lambda+\lambda_{1}\right) a^{3}+\left(\lambda^{2} \alpha-2 \lambda \lambda_{3}+2 \lambda_{1} \lambda_{3}+\lambda_{2}^{2}\right) a^{4}+\left(\lambda^{2} \theta+\right. \\
& \left.2 \lambda \lambda_{2} \gamma+2 \lambda \lambda_{4}+2 \lambda_{1} \lambda_{4}+2 \lambda_{2} \lambda_{3}\right) a^{5}, \\
a_{1}^{3}= & \lambda\left(\lambda_{1}^{2}-\lambda^{2}\right) a^{2} b+2 \lambda \lambda_{2}\left(\lambda+\lambda_{1}\right) a^{3} b+\lambda\left(\lambda^{2} \alpha-2 \lambda \lambda_{3}+2 \lambda_{1} \lambda_{3}+\lambda_{2}^{2}\right) a^{4} b+ \\
& \lambda_{1}\left(\lambda_{1}^{2}-\lambda^{2}\right) a^{3}+2 \lambda_{1} \lambda_{2}\left(\lambda+\lambda_{1}\right) a^{4}+\lambda_{1}\left(\lambda^{2} \alpha-2 \lambda \lambda_{3}+2 \lambda_{1} \lambda_{3}+\lambda_{2}^{2}\right) a^{5}+ \\
& \lambda_{2}\left(\lambda_{1}^{2}-\lambda^{2}\right) a^{4}+2 \lambda_{2} \lambda_{2}\left(\lambda+\lambda_{1}\right) a^{5}+\lambda_{3}\left(\lambda_{1}^{2}-\lambda^{2}\right) a^{5} \\
= & \left(\lambda_{1}-\lambda\right)\left(\lambda_{1}+\lambda^{2}\right) a^{3}+3 \lambda_{2}\left(\lambda_{1}-\lambda\right)\left(\lambda_{1}+\lambda\right) a^{4}+\left(\lambda_{1}+\lambda\right)\left(\lambda^{2} \alpha-\lambda_{2} \gamma-\right. \\
& \left.3 \lambda \lambda_{3}+\lambda \lambda_{1} \gamma+3 \lambda_{2}^{2}+3 \lambda_{1} \lambda_{3}\right) a^{5}, \\
a_{1}^{4}= & \lambda\left(\lambda_{1}-\lambda\right)\left(\lambda_{1}+\lambda^{2}\right) a^{3} b+3 \lambda \lambda_{2}\left(\lambda_{1}-\lambda\right)\left(\lambda_{1}+\lambda\right) a^{4} b+\lambda_{1}\left(\lambda_{1}-\lambda\right) \\
& \left(\lambda_{1}+\lambda^{2}\right) a^{4}+3 \lambda_{1} \lambda_{2}\left(\lambda_{1}-\lambda\right)\left(\lambda_{1}+\lambda\right) a^{5}+\lambda_{2}\left(\lambda_{1}-\lambda\right)\left(\lambda_{1}+\lambda^{2}\right) a^{5} \\
= & \left(\lambda-\lambda_{1}\right)^{2}\left(\lambda+\lambda_{1}\right)^{2} a^{4}-4 \lambda_{2}\left(\lambda-\lambda_{1}\right)\left(\lambda+\lambda_{1}\right)^{2} a^{5}, \\
a_{1}^{5}= & \lambda\left(\lambda-\lambda_{1}\right)^{2}\left(\lambda+\lambda_{1}\right)^{2} a^{4} b+\lambda_{1}\left(\lambda-\lambda_{1}\right)^{2}\left(\lambda+\lambda_{1}\right)^{2} a^{5} \\
= & \left(\lambda-\lambda_{1}\right)^{2}\left(\lambda+\lambda_{1}\right)^{3} a^{5},
\end{aligned}
$$

e uma vez que $a_{1}$ tem nilíndice máximo, segue que $\lambda \pm \lambda_{1} \neq 0$, portanto $\lambda \neq \pm \lambda_{1}$. Temos que

$$
\begin{aligned}
a_{1} b_{1}= & \lambda \beta b^{2}+\lambda \beta_{2} a^{2} b+\lambda \beta_{3} a^{3} b+\lambda \beta_{4} a^{4} b+\lambda_{1} \beta_{1} a^{2}+\lambda_{1} \beta_{2} a^{3}+\lambda_{1} \beta_{3} a^{4} \\
& +\lambda_{1} \beta_{4} a^{5}+\lambda_{2} \beta a^{2} b+\lambda_{2} \beta_{1} a^{3}+\lambda_{2} \beta_{2} a^{4}+\lambda_{2} \beta_{3} a^{5}+\lambda_{3} \beta a^{3} b+\lambda_{3} \beta_{1} a^{4} \\
& +\lambda_{3} \beta_{2} a^{5}+\lambda_{4} \beta a^{4} b+\lambda_{4} \beta_{1} a^{5}
\end{aligned}
$$




$$
\begin{aligned}
= & \left(-\lambda \beta+\lambda_{1} \beta_{1}\right) a^{2}+\left(\lambda \beta_{2}+\lambda_{1} \beta_{2}+\lambda_{2} \beta+\lambda_{2} \beta_{1}\right) a^{3}+\left(\lambda \beta \alpha-\lambda \beta_{3}\right. \\
& \left.+\lambda_{1} \beta_{3}+\lambda_{2} \beta_{2}-\lambda_{3} \beta+\lambda_{3} \beta_{1}\right) a^{4}+\left(\lambda \beta \theta+\lambda \beta_{2} \gamma+\lambda_{1} \beta_{4}+\lambda_{2} \beta \gamma\right. \\
& \left.+\lambda_{3} \beta_{2}+\lambda_{4} \beta+\lambda_{4} \beta_{1}+\lambda \beta_{4}+\lambda_{2} \beta_{3}\right) a^{5}=0 . \\
= & 0 .
\end{aligned}
$$

- $\left(\lambda_{1}=1\right.$ e $\lambda \neq \pm 1$.) Então

$$
\begin{aligned}
a_{1} b_{1}= & \left(-\lambda \beta+\beta_{1}\right) a^{2}+\left(\lambda \beta_{2}+\beta_{2}+\lambda_{2} \beta+\lambda_{2} \beta_{1}\right) a^{3}+\left(\lambda \beta \alpha-\lambda \beta_{3}\right. \\
& \left.+\beta_{3}+\lambda_{2} \beta_{2}-\lambda_{3} \beta+\lambda_{3} \beta_{1}\right) a^{4}+\left(\lambda \beta \theta+\lambda \beta_{2} \gamma+\beta_{4}+\lambda_{2} \beta \gamma\right. \\
& \left.+\lambda_{3} \beta_{2}+\lambda_{4} \beta+\lambda_{4} \beta_{1}+\lambda \beta_{4}+\lambda_{2} \beta_{3}\right) a^{5} \\
= & 0
\end{aligned}
$$

$\log 0 \beta_{1}=\lambda \beta$,

$$
\begin{gathered}
\beta_{2}=-\frac{\beta \lambda_{2}+\lambda_{2} \lambda \beta}{(\lambda+1)}=-\beta \lambda_{2}, \\
\beta_{3}=-\frac{\beta \lambda \alpha-\lambda_{2}^{2} \beta-\lambda_{3} \beta+\lambda_{3} \lambda \beta}{(1-\lambda)} \\
=\frac{\beta\left(\lambda \alpha-\lambda_{2}^{2}-\lambda_{3}+\lambda_{3} \lambda\right)}{(\lambda-1)},
\end{gathered}
$$

e

$$
\beta_{4}=-\frac{\beta\left(\lambda^{2} \theta-\lambda^{2} \lambda_{2} \gamma+2 \lambda \lambda_{2} \gamma+\lambda^{2} \lambda_{4}+\lambda_{2} \lambda \alpha-\lambda_{2}^{3}-\lambda \theta-\lambda_{2} \gamma-\lambda_{4}\right)}{\left(\lambda^{2}-1\right)} .
$$

Portanto

$$
\begin{aligned}
b_{1}= & \beta b+\lambda \beta a-\beta \lambda_{2} a^{2}+\frac{\beta\left(\lambda \alpha-\lambda_{2}^{2}-\lambda_{3}+\lambda_{3} \lambda\right)}{(\lambda-1)} a^{3} \\
& -\frac{\beta\left(\lambda^{2} \theta-\lambda^{2} \lambda_{2} \gamma+2 \lambda \lambda_{2} \gamma+\lambda^{2} \lambda_{4}+\lambda_{2} \lambda \alpha-\lambda_{2}^{3}-\lambda \theta-\lambda_{2} \gamma-\lambda_{4}\right)}{\left(\lambda^{2}-1\right)} a^{4} \\
& +\beta_{5} a^{5}
\end{aligned}
$$


com $\beta \neq 0$ e supondo $\beta=1$, temos que

$$
\begin{aligned}
b_{1}= & b+\lambda a-\lambda_{2} a^{2}+\frac{\left(\lambda \alpha-\lambda_{2}^{2}-\lambda_{3}+\lambda_{3} \lambda\right)}{(\lambda-1)} a^{3} \\
& -\frac{\lambda^{2} \theta-\lambda^{2} \lambda_{2} \gamma+2 \lambda \lambda_{2} \gamma+\lambda^{2} \lambda_{4}+\lambda_{2} \lambda \alpha-\lambda_{2}^{3}-\lambda \theta-\lambda_{2} \gamma-\lambda_{4}}{\left(\lambda^{2}-1\right)} a^{4} \\
& +\beta_{5} a^{5} .
\end{aligned}
$$

Logo

$$
\begin{aligned}
b_{1}^{2}= & b^{2}-2 \lambda_{2} a^{2} b+\frac{2\left(\lambda \alpha-\lambda_{2}^{2}-\lambda_{3}+\lambda_{3} \lambda\right)}{(\lambda-1)} a^{3} b+ \\
& \frac{2 \lambda^{2} \theta-\lambda^{2} \lambda_{2} \gamma+2 \lambda \lambda_{2} \gamma+\lambda^{2} \lambda_{4}+\lambda_{2} \lambda \alpha-\lambda_{2}^{3}-\lambda \theta-\lambda_{2} \gamma-\lambda_{4}}{\left(\lambda^{2}-1\right)} a^{4} b \\
& +\lambda^{2} a^{2}-2 \lambda \lambda_{2} a^{3}+\frac{2 \lambda\left(\lambda \alpha-\lambda_{2}^{2}-\lambda_{3}+\lambda_{3} \lambda\right)}{(\lambda-1)} a^{4}+ \\
& \frac{2 \lambda\left(\lambda^{2} \theta-\lambda^{2} \lambda_{2} \gamma+2 \lambda \lambda_{2} \gamma+\lambda^{2} \lambda_{4}+\lambda_{2} \lambda \alpha-\lambda_{2}^{3}-\lambda \theta-\lambda_{2} \gamma-\lambda_{4}\right)}{\left(\lambda^{2}-1\right)} a^{5} \\
& +\lambda_{2}^{2} a^{4}-\frac{2 \lambda_{2}\left(\lambda \alpha-\lambda_{2}^{2}-\lambda_{3}+\lambda_{3} \lambda\right)}{(\lambda-1)} a^{5} \\
= & \left(\lambda^{2}-1\right) a^{2}-2 \lambda_{2}(\lambda+1) a^{3}+\left[\frac{-2(\lambda+1)\left(\lambda \alpha-\lambda_{2}^{2}-\lambda_{3}+\lambda_{3} \lambda\right)}{(\lambda-1)}\right. \\
& \left.+\alpha+\lambda_{2}^{2}\right] a^{4}+\left[\theta-2 \lambda_{2} \gamma+\frac{2 \lambda_{2}\left(\lambda \alpha-\lambda_{2}^{2}-\lambda_{3}+\lambda_{3} \lambda\right)}{(\lambda-1)}+\right. \\
& \left.\frac{2(\lambda-1)\left(\lambda^{2} \theta-\lambda^{2} \lambda_{2} \gamma+2 \lambda \lambda_{2} \gamma+\lambda^{2} \lambda_{4}+\lambda_{2} \lambda \alpha-\lambda_{2}^{3}-\lambda \theta-\lambda_{2} \gamma-\lambda_{4}\right)}{\left(\lambda^{2}-1\right)}\right] a^{5} \\
= & \left(\lambda^{2}-1\right) a^{2}-2 \beta^{2} \lambda_{2}(1+\lambda) a^{3}-\left(-2 \lambda \alpha-2 \lambda \lambda_{3}-\alpha+2 \lambda_{3}+\lambda_{2}^{2}\right) a^{4} \\
+ & \left(-\lambda^{2} \theta-2 \lambda_{4} \lambda^{2}+2 \lambda^{2} \lambda_{2} \gamma-4 \lambda_{2} \lambda \alpha+3 \lambda \theta-6 \lambda \lambda_{2} \gamma+4 \lambda_{2}^{3}-\theta+4 \lambda_{2} \gamma\right. \\
+ & \left.+2 \lambda_{4}+2 \lambda_{2} \lambda_{3}-2 \lambda \lambda_{2} \lambda_{3}\right) a^{5}
\end{aligned}
$$


$\mathrm{e}$

$$
\begin{aligned}
a_{1}^{2} b_{1}= & \left(1-\lambda^{2}\right) a^{2} b+\left[\lambda^{2}+2 \lambda_{2}(\lambda+1)\right] a^{3} b+\lambda\left(1-\lambda^{2}\right) a^{3}+\lambda\left[\lambda^{2}+\right. \\
& \left.2 \lambda_{2}(\lambda+1)\right] a^{4}-\frac{\beta\left(\lambda+\lambda_{2}+\lambda_{2} \lambda\right)\left(1-\lambda^{2}\right)}{(\lambda+1)} a^{4} \\
= & (1+\lambda)\left(1-\lambda^{2}\right) a^{3}+\left[(\lambda-1)\left(\lambda^{2}+2 \lambda_{2} \lambda+2 \lambda_{2}\right)-(1-\lambda)(\lambda+\right. \\
& \left.\left.\lambda_{2}+\lambda_{2} \lambda\right)\right] a^{4} \\
= & -(1-\lambda)(1+\lambda)^{2} a^{3}+3 \lambda_{2}\left(\lambda^{2}-1\right)(\lambda+1)^{2} a^{4}+ \\
& (\lambda-1)\left(\lambda \alpha-\theta-3 \lambda_{3}\right) a^{5} .
\end{aligned}
$$

Seja $M$ a matriz cujas colunas são as coordenas de $a_{1}^{2}, a_{1}^{3}, a_{1}^{4}, a_{1}^{5}, b_{1}^{2}$ e $a_{1}^{2} b_{1}$ em termos das potências $a^{2}, a^{3}, a^{4}, a^{5}$. Fazendo uma redução por linhas de $M$ chegamos a matriz

$$
\left[\begin{array}{cccccc}
1 & 0 & 0 & 1 & -1 & 0 \\
0 & 1 & 0 & 0 & 0 & 1 \\
0 & 0 & 1 & 0 & \frac{\alpha}{(\lambda-1)^{2}} & \frac{6 \lambda_{2}}{\left(\lambda^{2}-1\right)} \\
0 & 0 & 0 & 1 & \frac{\mu_{1}}{(\lambda-1)^{3}(\lambda+1)^{2}} & \frac{\mu_{2}}{\left(\lambda^{2}-1\right)^{2}}
\end{array}\right],
$$

$\operatorname{com} \mu_{1}=-3 \lambda^{2} \theta+4 \lambda^{2} \lambda_{2} \gamma+4 \lambda_{2} \lambda \alpha+3 \lambda \theta-8 \lambda \lambda_{2} \gamma+4 \lambda_{2}^{3}-\theta+4 \lambda_{2} \gamma+$ $\lambda^{3} \theta+4 \lambda_{2} \alpha+4 \lambda_{2} \alpha \lambda^{2}$ e $\mu_{2}=\lambda^{2} \gamma-2 \lambda \gamma-\lambda \alpha+\gamma+21 \lambda_{2}^{2}$.

Portanto utilizando o Lema 4.17, temos que para $\alpha^{\prime}, \theta^{\prime}$ e $\gamma^{\prime}$ em $F$, os produtos $b_{1}^{2}$ e $a_{1}^{2} b_{1}$, são da forma $-a_{1}^{2}+\alpha^{\prime} a^{4}+\theta^{\prime} a^{5}$ e $a_{1}^{3}+\gamma^{\prime} a_{1}^{5}$, respectivamente se, e somente se,

$$
\begin{aligned}
& \frac{x^{2}}{y^{4}} \frac{\alpha}{(\lambda-1)^{2}}=\alpha^{\prime}, \quad \frac{x^{2}}{y^{5}} \frac{\mu_{1}}{(\lambda-1)^{3}(\lambda+1)^{2}}=\theta^{\prime}, \\
& \frac{x}{y}=1, \quad \frac{x}{y^{2}} \frac{6 \lambda_{2}}{\left(\lambda^{2}-1\right)}=0 \text { e } \frac{x}{y^{3}} \frac{\mu_{2}}{\left(\lambda^{2}-1\right)^{2}}=\gamma^{\prime},
\end{aligned}
$$

para $x, y$ não nulos. Logo $x=y, \quad \lambda_{2}=0$ e assim $\mu_{1}=-3 \lambda^{2} \theta+3 \lambda \theta-$ $\theta+\lambda^{3} \theta$ e $\mu_{2}=\lambda^{2} \gamma-2 \lambda \gamma-\lambda \alpha+\gamma$, de onde

$$
\alpha^{\prime}=\frac{\alpha}{y^{2}(\lambda-1)^{2}}, \quad \theta^{\prime}=\frac{\left(-3 \lambda^{2}+3 \lambda-1+\lambda^{3}\right) \theta}{y^{3}(\lambda-1)^{3}(\lambda+1)^{2}}=\frac{\theta}{y^{3}(\lambda+1)^{3}},
$$


$\mathrm{e}$

$$
\begin{aligned}
\gamma^{\prime} & =\frac{\left(\lambda^{2}-2 \lambda+1\right) \gamma-\lambda \alpha}{y^{2}\left(\lambda^{2}-1\right)^{2}}=\frac{1}{y^{2}(\lambda+1)^{2}}\left(\gamma-\frac{\lambda \alpha}{(\lambda-1)^{2}}\right) \\
& =\frac{1}{y^{2}(\lambda+1)^{2}}\left(\gamma-\lambda \alpha^{\prime}\right)
\end{aligned}
$$

Lembrando que $y \neq 0$ e $\lambda \neq \pm 1$, podemos definir $\rho_{1}, \rho_{2} \neq 0$, assim $\rho_{1}=y(\lambda-1)$ e $\rho_{2}=y(\lambda+1)$. Portanto

$$
\alpha^{\prime}=\frac{\alpha}{\rho_{1}^{2}}, \quad \theta^{\prime}=\frac{\theta}{\rho_{2}^{3}}, \quad \gamma^{\prime}=\frac{1}{\rho_{2}^{2}}\left(\gamma+\frac{\rho_{1}+\rho_{2}}{\rho_{1}-\rho_{2}} \alpha^{\prime}\right) .
$$

- $\left(\lambda=1\right.$ e $\lambda_{1}=0$. $)$ Então

$$
\begin{aligned}
a_{1} b_{1}= & -\beta a^{2}+\left(\beta_{2}+\lambda_{2} \beta+\lambda_{2} \beta_{1}\right) a^{3}+\left(\beta \alpha-\beta_{3}+\lambda_{2} \beta_{2}-\lambda_{3} \beta+\right. \\
& \left.\lambda_{3} \beta_{1}\right) a^{4}+\left(\beta \theta+\beta_{2} \gamma+\lambda_{2} \beta \gamma+\lambda_{3} \beta_{2}+\lambda_{4} \beta+\lambda_{4} \beta_{1}+\beta_{4}+\right. \\
& \left.\lambda_{2} \beta_{3}\right) a^{5} \\
= & 0 .
\end{aligned}
$$

$\operatorname{logo} \beta=0, \beta_{2}=-\beta_{1} \lambda_{2}, \beta_{3}=-\beta_{1}\left(\lambda_{3}-\lambda_{2}^{2}\right)$ e $\beta_{4}=-\beta_{1}\left(-\lambda_{2} \gamma+\lambda_{4}-\right.$ $\left.\lambda_{2}^{2}\right)$, portanto

$$
b_{1}=\beta_{1} a-\beta_{1} \lambda_{2} a^{2}-\beta_{1}\left(\lambda_{3}-\lambda_{2}^{2}\right) a^{3}-\beta_{1}\left(-\lambda_{2} \gamma+\lambda_{4}-\lambda_{2}^{2}\right) a^{4}+\beta_{5} a^{5},
$$

com $\beta_{1} \neq 0$, e supondo $\beta=1$, temos que

$$
b_{1}=a-\lambda_{2} a^{2}-\left(\lambda_{3}-\lambda_{2}^{2}\right) a^{3}-\left(-\lambda_{2} \gamma+\lambda_{4}-\lambda_{2}^{2}\right) a^{4}+\beta_{5} a^{5} .
$$

Portanto

$$
b_{1}^{2}=a^{2}-2 \lambda_{2} a^{3}+\left(2 \lambda_{3}-\lambda_{2}^{2}\right) a^{4}+2\left(\lambda_{2} \gamma-\lambda_{4}+2 \lambda_{2}^{3}-\lambda_{2} \lambda_{3}\right) a^{5},
$$

e

$$
a_{1}^{2} b_{1}=-a^{3}+3 \lambda_{2} a^{4}+\left(\alpha-3 \lambda_{3}\right) a^{5},
$$


e temos a matriz

$$
\left[\begin{array}{cccccc}
-1 & 0 & 0 & 0 & 1 & 0 \\
2 \lambda_{2} & -1 & 0 & 0 & -2 \lambda_{2} & -1 \\
\alpha-\mu_{1} & -3 \lambda_{2} & 1 & 0 & \mu_{2} & 3 \lambda_{2} \\
\theta+\mu_{3} & \alpha-\gamma-\mu_{4} & -4 \lambda_{2} & 1 & 2 \mu_{5} & \left(\alpha-3 \lambda_{3}\right)
\end{array}\right]
$$

com $\mu_{1}=2 \lambda_{3}+\lambda_{2}^{2}, \mu_{2}=\left(2 \lambda_{3}-\lambda_{2}^{2}\right), \mu_{3}=2 \lambda_{2}\left(\gamma+\lambda_{3}\right)+2 \lambda_{4}, \mu_{4}=$ $3\left(\lambda_{3}+\lambda_{2}^{2}\right)$ e $\mu_{5}=\lambda_{2} \gamma-\lambda_{4}+2 \lambda_{2}^{3}-\lambda_{2} \lambda_{3}$. Reduzindo por linhas a matriz anterior obtemos

$$
\left[\begin{array}{cccccc}
1 & 0 & 0 & 0 & -1 & 0 \\
0 & 1 & 0 & 0 & 0 & 1 \\
0 & 0 & 1 & 0 & 1 \alpha & 6 \lambda_{2} \\
0 & 0 & 0 & 1 & 4 \lambda_{2}^{3}+4 \lambda_{2} \gamma+\theta+4 \lambda_{2} \alpha & \gamma+21 \lambda_{2}
\end{array}\right] .
$$

Portanto os produtos $b_{1}^{2}$ e $a_{1}^{2} b_{1}$, são da forma $-a_{1}^{2}+\alpha^{\prime} a^{4}+\theta^{\prime} a^{5}$ e $a_{1}^{3}+\gamma^{\prime} a_{1}^{5}$, para $\alpha^{\prime}, \theta^{\prime}$ e $\gamma^{\prime}$ em $F$, se e somente se,

$$
\alpha=\alpha^{\prime}, \quad 4 \lambda_{2}^{3}+4 \lambda_{2} \gamma+\theta+4 \lambda_{2} \alpha=\theta^{\prime}, \quad 6 \lambda_{2}=0 \text { e } \gamma+21 \lambda_{2}=\gamma^{\prime},
$$

$\operatorname{logo} \lambda_{2}=0$, e assim $\alpha^{\prime}=\alpha, \theta^{\prime}=\theta$ e $\gamma^{\prime}=\gamma$. Isto demonstra o lema.

Teorema 4.26 Uma nilálgebra comutativa de p.a. de dimensão e nilíndice 6, é isomorfa a uma e somente uma álgebra com base $\left\{b, a, a^{2}, a^{3}, a^{4}, a^{5}\right\}$, e produtos não triviais e não zero conforme um dos casos que aparecem na seguinte lista:
$\mathcal{A}_{1}(\alpha, \theta, \gamma): \quad b^{2}=-a^{2}+\alpha a^{4}+\theta a^{5}, \quad a^{2} b=a^{3}+\gamma a^{5}, \quad a^{3} b=-a^{4}, a^{4} b=a^{5} ;$
$\mathcal{A}_{2}(\alpha): \quad b^{2}=\alpha a^{4}$,
$\left[\rho^{2}\right] ;$
$\mathcal{A}_{3}(\alpha): \quad b^{2}=\alpha a^{4}$,
$a^{2} b=a^{4}, \quad a^{3} b=-a^{5}$,
[1]; 


\begin{tabular}{|c|c|c|c|}
\hline $\mathcal{A}_{4}:$ & $b^{2}=-3 a^{4}$ & $a^{2} b=a^{4}$ & $a^{3} b=-a^{5}$ \\
\hline $\mathcal{A}_{5}:$ & $b^{2}=-3 a^{4}+a^{5}$ & $a^{2} b=a^{4}$ & $a^{3} b=-a^{5}$ \\
\hline $\mathcal{A}_{6}:$ & $b^{2}=a^{5}$ & & \\
\hline $\mathcal{A}_{7}:$ & $b^{2}=a^{5}$ & $a^{2} b=a^{4}$ & $a^{3} b=-a^{5}$ \\
\hline $\mathcal{A}_{8}:$ & $b^{2}=a^{5}$ & $a^{2} b=a^{5}$ & \\
\hline $\mathcal{A}_{9}:$ & & $a^{2} b=a^{4}$ & $a^{3} b=-a^{5}$ \\
\hline $\mathcal{A}_{10}:$ & & $a^{2} b=a^{5}$ & \\
\hline
\end{tabular}

sendo que $\mathcal{A}_{1}(\alpha, \theta, \gamma) \cong \mathcal{A}_{1}\left(\alpha^{\prime}, \theta^{\prime}, \gamma^{\prime}\right)$ se, e somente se, existirem $\rho_{1}, \rho_{2} \in F^{*}$ tal que

$$
\alpha^{\prime}=\frac{\alpha}{\rho_{1}^{2}}, \quad \theta^{\prime}=\frac{\theta}{\rho_{2}^{3}} \quad \text { e } \quad \gamma^{\prime}=\frac{1}{\rho_{2}^{2}}\left(\gamma+\frac{\rho_{1}+\rho_{2}}{\rho_{1}-\rho_{2}} \alpha^{\prime}\right) .
$$

Além disso, temos que para $\mathcal{A}_{3}(\alpha), \alpha$ é diferente de zero e menos três.

Observamos que a matriz da direita dá a condição suficiente e necessária par que duas álgebras desta classe sejam isomorfas, no caso da matriz [1], temos que $\mathcal{A}_{3}(\alpha) \cong \mathcal{A}_{3}\left(\alpha^{\prime}\right)$ se, e somente se, $\alpha=\alpha^{\prime}$.

\subsection{Classificação para dimensão sete.}

Nesta seção classificaremos, a menos de isomorfismos, todas as nilálgebras comutativas de potências associativas de dimensão e nilíndice 7. O método utilizado será basicamente o mesmo utilizado para dimensão cinco. Utilizaremos também alguns resultados da seção anterior.

Teorema 4.27 Seja $\mathcal{B}$ uma nilálgebra comutativa de p.a. de dimensão e nilíndice 7 e $(a, b)$ um elemento de $\mathcal{P}(\mathcal{B})$. Então os produtos não triviais, 
para a base determinada pelo par $(a, b)$, são dados por:

$$
\begin{aligned}
b^{2} & =\gamma_{4}^{2} a^{4}+\alpha_{5} a^{5}+\alpha_{6} a^{6}, \\
a^{2} b & =\gamma_{4} a^{4}+\gamma_{5} a^{5}+\gamma_{6} a^{6}, \\
a^{3} b & =-\gamma_{4} a^{5}-\gamma_{5} a^{6}, \\
a^{4} b & =\gamma_{4} a^{6} .
\end{aligned}
$$

Reciprocamente, se $\mathcal{B}$ é uma nilálgebra comutativa com base $\left\{b, a, a^{2}, \ldots, a^{6}\right\}$, $e$ produtos $a b=a^{k}=a b=0, a^{i} a^{j}=a^{i+j}$, para $k \geq 7$ e $i, j \geq 1$ e (4.8), então $\mathcal{B}$ é de p.a. e de nilíndice 7.

Prova: Seja $\mathcal{B}$ uma nilálgebra comutativa de p.a. de dimensão e nilíndice 7 e $(a, b)$ em $\mathcal{P}(\mathcal{B})$. Então, $\overline{\mathcal{B}}=\mathcal{B} / A_{a}^{6}=\left\langle\bar{b}, \bar{a}, \bar{a}^{2}, \bar{a}^{3}, \bar{a}^{4}, \bar{a}^{5}\right\rangle$ e pelo Teorema 4.16 temos que $\bar{b}^{2}=-\gamma_{3}^{2} \bar{a}^{2}+\alpha_{4} \bar{a}^{4}+\alpha_{5} \bar{a}^{5}$ e $\bar{a}^{2} \bar{b}=\gamma_{3} \bar{a}^{3}+\gamma_{4} \bar{a}^{4}+\gamma_{5} \bar{a}^{5}$, logo

$$
b^{2}=-\gamma_{3}^{2} a^{2}+\alpha_{4} a^{4}+\alpha_{5} a^{5}+\alpha_{6} a^{6} \quad \text { e } \quad a^{2} b=\gamma_{3} a^{3}+\gamma_{4} a^{4}+\gamma_{5} a^{5}+\gamma_{6} a^{6}
$$

e utilizando (4.2), (4.3) e (4.4), obtemos que

$$
\begin{aligned}
a^{3} b & =-\gamma_{3} a^{4}-\gamma_{4} a^{5}-\gamma_{5} a^{6} \\
a^{4} b & =\frac{1}{3}\left[3\left(\gamma_{3} a^{5}+\gamma_{4} a^{6}\right)-2\left(-\gamma_{3} a^{5}-\gamma_{4} a^{6}\right)-2\left(\gamma_{3} a^{5}+\gamma_{4} a^{6}\right)\right]=\gamma_{3} a^{5}+\gamma_{4} a^{6}, \\
a^{5} b & =\frac{1}{3}\left[4\left(-\gamma_{3} a^{6}\right)-\left(\gamma_{3} a^{6}\right)-2\left(\gamma_{3} a^{6}\right)-2\left(-\gamma_{3} a^{6}\right)\right]=-\frac{5}{3} \gamma_{3} a^{6}, \\
\text { e de }(4.1), & \\
a^{2} b^{2} & =\frac{1}{2}\left[\left(-\gamma_{3}^{2} a^{4}+\alpha_{4} a^{6}\right)+\left(\gamma_{3} a^{3} b+\gamma_{4} a^{4} b+\gamma_{5} a^{5} b\right)\right] \\
& =\frac{1}{2}\left[-\gamma_{3}^{2} a^{4}+\alpha_{4} a^{6}-\gamma_{3}^{2} a^{4}-\gamma_{3} \gamma_{4} a^{5}-\gamma_{3} \gamma_{5} a^{6}+\gamma_{4} \gamma_{3} a^{5}+\gamma_{4}^{2} a^{6}-\frac{5}{3} \gamma_{3} \gamma_{5} a^{6}\right] \\
& =\frac{1}{2}\left[-2 \gamma_{3}^{2} a^{4}+\left(\alpha_{4}+\gamma_{4}^{2}-\frac{8}{3} \gamma_{3} \gamma_{5}\right) a^{6}\right],
\end{aligned}
$$

mas $a^{2} b^{2}=-\gamma_{3}^{2} a^{4}+\alpha_{4} a^{6}, \operatorname{logo} \alpha_{4}=\gamma_{4}^{2}-\frac{8}{3} \gamma_{3} \gamma_{5}$ e portanto

$$
b^{2}=-\gamma_{3}^{2} a^{2}+\left(\gamma_{4}^{2}-\frac{8}{3} \gamma_{3} \gamma_{5}\right) a^{4}+\alpha_{5} a^{5}+\alpha_{6} a^{6} .
$$


Uma vez que $\mathcal{B}$ é de p.a. temos que para qualquer elemento $x \in \mathcal{B}$, cumpre-se que $x^{4}=x^{2} x^{2}$. Seja $x=b+a^{2}$. Então

$$
\begin{aligned}
& x^{2}=-\gamma_{3}^{2} a^{2}+2 \gamma_{3} a^{3}+\left(\gamma_{4}^{2}-\frac{8}{3} \gamma_{3} \gamma_{5}+2 \gamma_{4}+1\right) a^{4}+\left(2 \gamma_{5}+\alpha_{5}\right) a^{5}+\left(2 \gamma_{6}+\alpha_{6}\right) a^{6}, \\
& x^{3}=-\gamma_{3}^{3} a^{3}+\left(-\gamma_{3}^{2} \gamma_{4}-3 \gamma_{3}^{2}\right) a^{4}+\left(-\frac{11}{3} \gamma_{3}^{2} \gamma_{5}+\gamma_{3} \gamma_{4}^{2}+3 \gamma_{3}\right) a^{5}+\left(-\gamma_{3}^{2} \gamma_{6}-8 \gamma_{3} \gamma_{5}\right. \\
& \left.+\gamma_{4}^{3}-\frac{8}{3} \gamma_{3} \gamma_{4} \gamma_{5}+3 \gamma_{4}^{2}+3 \gamma_{4}-\frac{5}{3} \gamma_{3} \alpha_{5}+1\right) a^{6} \\
& x^{4}=\gamma_{3}^{4} a^{4}-4 \gamma_{3}^{3} a^{5}+\left(\frac{64}{9} \gamma_{3}^{3} \gamma_{5}-\frac{8}{3} \gamma_{3}^{2} \gamma_{4}^{2}-4 \gamma_{3}^{2} \gamma_{4}-8 \gamma_{3}^{2}\right) a^{6} \\
& x^{2} x^{2}=\gamma_{3}^{4} a^{4}-4 \gamma_{3}^{3} a^{5}+\left(-2 \gamma_{3}^{2} \gamma_{4}^{2}+\frac{16}{3} \gamma_{3}^{3} \gamma_{5}-4 \gamma_{3}^{2} \gamma_{4}-8 \gamma_{3}^{2}\right) a^{6},
\end{aligned}
$$

$\log 0$

$$
0=x^{4}-x^{2} x^{2}=\left(\frac{16}{9} \gamma_{3}^{3} \gamma_{5}-\frac{2}{3} \gamma_{3}^{2} \gamma_{4}^{2}-10 \gamma_{3}^{2}\right) a^{6},
$$

e portanto

$$
\frac{2}{9} \gamma_{3}^{2}\left(8 \gamma_{3} \gamma_{5}-3 \gamma_{4}^{2}-45\right)=0
$$

Seja agora $y=3 b+a^{2}$. Então

$$
\begin{aligned}
& y^{2}=-9 \gamma_{3}^{2} a^{2}+6 \gamma_{3} a^{3}+\left(9 \gamma_{4}^{2}-24 \gamma_{3} \gamma_{5}+6 \gamma_{4}+1\right) a^{4} \\
&+\left(6 \gamma_{5}+9 \alpha_{5}\right) a^{5}+\left(6 \gamma_{6}+9 \alpha_{6}\right) a^{6}, \\
& y^{3}=-27 \gamma_{3}^{3} a^{3}+\left(-27 \gamma_{3}^{2} \gamma_{4}-27 \gamma_{3}^{2}\right) a^{4}+\left(-99 \gamma_{3}^{2} \gamma_{5}+27 \gamma_{3} \gamma_{4}^{2}+9 \gamma_{3}\right) a^{5} \\
&+\left(-27 \gamma_{3}^{2} \gamma_{6}-72 \gamma_{3} \gamma_{5}+27 \gamma_{4}^{3}-72 \gamma_{3} \gamma_{4} \gamma_{5}+27 \gamma_{4}^{2}+9 \gamma_{4}-45 \gamma_{3} \alpha_{5}+1\right) a^{6}, \\
& y^{4}= 81 \gamma_{3}^{4} a^{4}-108 \gamma_{3}^{3} a^{5}+\left(576 \gamma_{3}^{3} \gamma_{5}-216 \gamma_{3}^{2} \gamma_{4}^{2}-108 \gamma_{3}^{2} \gamma_{4}-72 \gamma_{3}^{2}\right) a^{6} \\
& \mathrm{e}
\end{aligned}
$$

$$
y^{2} y^{2}=81 \gamma_{3}^{4} a^{4}-108 \gamma_{3}^{3} a^{5}+\left(-162 \gamma_{3}^{2} \gamma_{4}^{2}+432 \gamma_{3}^{3} \gamma_{5}-108 \gamma_{3}^{2} \gamma_{4}+18 \gamma_{3}^{2}\right) a^{6},
$$

$\log 0$

$$
0=y^{4}-y^{2} y^{2}=\left(144 \gamma_{3}^{3} \gamma_{5}-54 \gamma_{3}^{2} \gamma_{4}^{2}-90 \gamma_{3}^{2}\right) a^{6},
$$


e portanto

$$
18 \gamma_{3}^{2}\left(8 \gamma_{3} \gamma_{5}-3 \gamma_{4}^{2}-5\right)=0
$$

Se $\gamma_{3} \neq 0$, então de (4.9) temos que, $\gamma_{5}=\frac{1}{8 \gamma_{3}}\left(3 \gamma_{4}^{2}+45\right)$, e por $(4.10)$ temos que, $\gamma_{5}=\frac{1}{8 \gamma_{3}}\left(3 \gamma_{4}^{2}+5\right)$, o que nos leva a um absurdo. Portanto $\gamma_{3}=0$ e assim $b^{2}=\gamma_{4}^{2} a^{4}+\alpha_{5} a^{5}+\alpha_{6} a^{6}, a^{2} b=\gamma_{4} a^{4}+\gamma_{5} a^{5}+\gamma_{6} a^{6}, a^{3} b=-\gamma_{4} a^{5}-\gamma_{5} a^{6}$, $a^{4} b=\gamma_{4} a^{6}$ e $a^{5} b=0$.

Suponhamos agora que $\mathcal{B}$ é uma nilálgebra comutativa, com base $\left\{b, a, a^{2}\right.$, $\left.a^{3}, a^{4}, a^{5}, a^{6}\right\}$, e produtos dados por $a b=a^{k}=a b=0, a^{i} a^{j}=a^{i+j}$, para $k \geq 6, i, j \geq 1$ e (4.8). Vejamos que $\mathcal{B}$ é de p.a. e tem nilíndice 7. Para tal seja $x=\lambda b+\sum_{i=1}^{6} \lambda_{i} a^{i}$. Então

$$
\begin{aligned}
x^{2}= & \lambda_{1}^{2} a^{2}+2 \lambda_{1} \lambda_{2} a^{3}+\left(\lambda^{2} \gamma_{4}^{2}+2 \lambda \lambda_{2} \gamma_{4}+2 \lambda_{1} \lambda_{3}+\lambda_{2}^{2}\right) a^{4}+\left(\lambda^{2} \alpha_{5}+2 \lambda \lambda_{2} \gamma_{5}\right. \\
& \left.-2 \lambda \lambda_{3} \gamma_{4}+2 \lambda_{1} \lambda_{4}+2 \lambda_{2} \lambda_{3}\right) a^{5}+\left(\lambda^{2} \alpha_{6}+2 \lambda \lambda_{2} \gamma_{6}-2 \lambda \lambda_{3} \gamma_{5}+2 \lambda \lambda_{4} \gamma_{4}\right. \\
& \left.+2 \lambda_{1} \lambda_{5}+2 \lambda_{2} \lambda_{4}+\lambda_{3}^{2}\right) a^{6}, \\
x^{3}= & \lambda_{1}^{3} a^{3}+\left(\lambda \lambda_{1}^{2} \gamma_{4}+3 \lambda_{1}^{2} \lambda_{2}\right) a^{4}+\left(\lambda \lambda_{1}^{2} \gamma_{5}+\lambda^{2} \lambda_{1} \gamma_{4}^{2}+3 \lambda_{3} \lambda_{1}^{2}+3 \lambda_{1} \lambda_{2}^{2}\right) a^{5} \\
& +\left(\lambda \lambda_{1}^{2} \gamma_{6}+\lambda^{3} \gamma_{4}^{3}+3 \lambda^{2} \lambda_{2} \gamma_{4}^{2}+3 \lambda \lambda_{2}^{2} \gamma_{4}+\lambda_{1} \lambda^{2} \alpha_{5}+3 \lambda_{1}^{2} \lambda_{4}+6 \lambda_{1} \lambda_{2} \lambda_{3}\right. \\
& \left.+\lambda_{2}^{3}\right) a^{6}, \\
x^{4}= & \lambda_{1}^{4} a^{4}+4 \lambda_{1}^{3} \lambda_{2} a^{5}+\left(2 \lambda^{2} \lambda_{1}^{2} \gamma_{4}^{2}+4 \lambda \lambda_{1}^{2} \lambda_{2} \gamma_{4}+4 \lambda_{1}^{3} \lambda_{3}+6 \lambda_{1}^{2} \lambda_{2}^{2}\right) a^{6}, \quad(4.11) \\
x^{5}= & \lambda_{1}^{5} a^{5}+\left(\lambda \lambda_{1}^{4} \gamma_{4}+5 \lambda_{1}^{4} \lambda_{2}\right) a^{6}, \\
x^{6}= & \lambda_{1}^{6} a^{6}, \\
x^{7}= & 0, \\
x^{2} x^{2}= & \lambda_{1}^{4} a^{4}+4 \lambda_{1}^{3} \lambda_{2} a^{5}+\left(2 \lambda^{2} \lambda_{1}^{2} \gamma_{4}^{2}+4 \lambda \lambda_{1}^{2} \lambda_{2} \gamma_{4}+4 \lambda_{1}^{3} \lambda_{3}+6 \lambda_{1}^{2} \lambda_{2}^{2}\right) a^{6},
\end{aligned}
$$

assim $x^{4}=x^{2} x^{2}$ e podemos concluir que $\mathcal{B}$ é de p.a. e tem nilíndice 7.

Observamos que os produtos dados por (4.11), dão a expressão geral para as potências de um elemento qualquer da álgebra do lema anterior. Utilizaremos estes produtos indicando os valores dos escalares $\alpha_{i}$ e $\gamma_{i}$, cada vez que fomos calcular as potências de elementos desta álgebra neste tipo de base. 
Denotaremos a álgebra do lema anterior por $\mathcal{B}\left(\alpha_{5}, \alpha_{6}, \gamma_{5}, \gamma_{6}, \gamma_{7}\right)$. A matriz coordenada por linha, do operador $L_{b}$, na base $\left\{b, a, a^{2}, a^{3}, a^{4}, a^{5}, a^{6}\right\}$ é

$$
\left(\begin{array}{ccccccc}
0 & 0 & 0 & 0 & \gamma_{4}^{2} & \alpha_{5} & \alpha_{6} \\
0 & 0 & 0 & 0 & 0 & 0 & 0 \\
0 & 0 & 0 & 0 & \gamma_{4} & \gamma_{5} & \gamma_{6} \\
0 & 0 & 0 & 0 & 0 & -\gamma_{4} & -\gamma_{5} \\
0 & 0 & 0 & 0 & 0 & 0 & \gamma_{4} \\
0 & 0 & 0 & 0 & 0 & 0 & 0 \\
0 & 0 & 0 & 0 & 0 & 0 & 0
\end{array}\right)
$$

Estudemos as classes de isomorfismos de todas as álgebras da forma $\mathcal{B}\left(\alpha_{5}, \alpha_{6}, \gamma_{4}, \gamma_{5}, \gamma_{6}\right)$.

Lema 4.28 Sejam $\lambda$ e $\beta$ em $F^{*}$. Então

$$
\mathcal{B}\left(\alpha_{5}, \alpha_{6}, \gamma_{4}, \gamma_{5}, \gamma_{6}\right) \cong \mathcal{B}\left(\frac{\beta^{2}}{\lambda^{5}} \alpha_{5}, \frac{\beta^{2}}{\lambda^{6}} \alpha_{6}, \frac{\beta}{\lambda^{2}} \gamma_{4}, \frac{\beta}{\lambda^{3}} \gamma_{5}, \frac{\beta}{\lambda^{4}} \gamma_{6}\right)
$$

Prova: Seja $\mathcal{B}$ a álgebra $\mathcal{B}\left(\alpha_{5}, \alpha_{6}, \gamma_{4}, \gamma_{5}, \gamma_{6}\right)$, com base determinada pelo par $(a, b)$. Definimos os elementos $a_{1}:=\lambda a$ e $b_{1}:=\beta b$, para $\lambda$ e $\beta$ em $F^{*}$. Claramente $\left(a_{1}, b_{1}\right)$ pertence a $\mathcal{P}(\mathcal{B})$. Determinemos as constantes de estrutura de $\mathcal{B}$ em relação a base determinada por $\left(a_{1}, b_{1}\right)$. Uma vez que $a_{1}^{4}=\lambda^{4} a^{4}, a_{1}^{5}=\lambda^{5} a^{5}$ e $a_{1}^{6}=\lambda^{6} a^{6}$, segue que

$$
\begin{aligned}
b_{1}^{2} & =\beta^{2} b^{2}=\beta^{2} \gamma_{4}^{2} \frac{1}{\lambda^{4}} a_{1}^{4}+\beta^{2} \alpha_{5} \frac{1}{\lambda^{5}} a_{1}^{5}+\beta^{2} \alpha_{6} \frac{1}{\lambda^{6}} a_{1}^{6}, \\
a_{1}^{2} b_{1} & =\lambda^{2} \beta a^{2} b=\beta \gamma_{4} \frac{1}{\lambda^{2}} a_{1}^{4}+\beta \gamma_{5} \frac{1}{\lambda^{3}} a_{1}^{5}+\beta \gamma_{6} \frac{1}{\lambda^{4}} a_{1}^{6},
\end{aligned}
$$

o que demonstra o lema.

Lema 4.29 Sejam $\rho_{1}$ e $\rho_{2}$ em F. Então a álgebra $\mathcal{B}\left(\alpha_{5}, \alpha_{6}, \gamma_{4}, \gamma_{5}, \gamma_{6}\right)$ é isomorfa à álgebra

$$
\mathcal{B}\left(\alpha_{5}-2 \rho_{1} \gamma_{4}^{2}, \alpha_{6}-2 \rho_{2} \gamma_{4}^{2}+7 \rho_{1}^{2} \gamma_{4}^{2}-5 \rho_{1} \alpha_{5}, \gamma_{4}, \gamma_{5}-7 \rho_{1} \gamma_{4}, \gamma_{6}+\theta\right)
$$

sendo que $\theta=-8 \rho_{1} \gamma_{5}-\rho_{2} \gamma_{4}+29 \rho_{1}^{2} \gamma_{4}$. 
Prova: Seja $\mathcal{B}$ a álgebra $\mathcal{B}\left(\alpha_{5}, \alpha_{6}, \gamma_{4}, \gamma_{5}, \gamma_{6}\right)$ com base $\left\{b, a, a^{2}, a^{3}, a^{4}, a^{5}, a^{6}\right\}$. Sejam $\rho_{1}$ e $\rho_{2}$ em $F$,

$$
a_{1}:=a+\rho_{1} a^{2}+\rho_{2} a^{3}
$$

e

$$
b_{1}:=b-\rho_{1} \gamma_{4} a^{3}+\left(-\rho_{1} \gamma_{5}+\rho_{1}^{2} \gamma_{4}+\rho_{2} \gamma_{4}\right) a^{4}-\left(\rho_{1} \gamma_{6}-\rho_{2} \gamma_{5}-\rho_{1}^{2} \gamma_{5}+\rho_{1}^{3} \gamma_{4}\right) a^{5} .
$$

Então

$$
\begin{aligned}
& a_{1}^{2}=a^{2}+2 \rho_{1} a^{3}+\left(2 \rho_{2}+\rho_{1}^{2}\right) a^{4}+2 \rho_{1} \rho_{2} a^{5}+\rho_{2}^{2} a^{6}, \\
& a_{1}^{3}=a^{3}+3 \rho_{1} a^{4}+3\left(\rho_{2}+\rho_{1}^{2}\right) a^{5}+\rho_{1}\left(6 \rho_{2}+\rho_{1}^{2}\right) a^{6}, \\
& a_{1}^{4}=a^{4}+4 \rho_{1} a^{5}+\left(4 \rho_{2}+6 \rho_{1}^{2}\right) a^{6}, \\
& a_{1}^{5}=a^{5}+5 \rho_{1} a^{6}, \\
& a_{1}^{6}=a^{6} \neq 0
\end{aligned}
$$

e

$$
\begin{aligned}
a_{1} b_{1}= & -\rho_{1} \gamma_{4} a^{4}+\left(-\rho_{1} \gamma_{5}+\rho_{1}^{2} \gamma_{4}+\rho_{2} \gamma_{4}\right) a^{5}-\left(\rho_{1} \gamma_{6}-\rho_{2} \gamma_{5}-\rho_{1}^{2} \gamma_{5}+\rho_{1}^{3} \gamma_{4}\right) a^{6} \\
& +\rho_{1} a^{2} b-\rho_{1}^{2} \gamma_{4} a^{5}+\rho_{1}\left(-\rho_{1} \gamma_{5}+\rho_{1}^{2} \gamma_{4}+\rho_{2} \gamma_{4}\right) a^{6}+\rho_{2} a^{3} b \rho_{1} \rho_{2} \gamma_{4} a^{6} \\
= & \left(-\rho_{1} \gamma_{4}+\rho_{1} \gamma_{4}\right) a^{4}+\left(-\rho_{1} \gamma_{5}+\rho_{1}^{2} \gamma_{4}+\rho_{2} \gamma_{4}-\rho_{1}^{2} \gamma_{4}+\rho_{1} \gamma_{5}-\rho_{2} \gamma_{4}\right) a^{5}+ \\
& \left(-\rho_{1} \gamma_{6}+\rho_{2} \gamma_{5}+\rho_{1}^{2} \gamma_{5}-\rho_{1}^{3} \gamma_{4}+\rho_{1} \gamma_{6}-\rho_{1}^{2} \gamma_{5}+\rho_{1}^{3} \gamma_{4}+\rho_{1} \rho_{2} \gamma_{4}-\rho_{2} \gamma_{5}\right. \\
& \left.-\rho_{1}^{3} \rho_{2} \gamma_{5}\right) a^{6} \\
= & 0 .
\end{aligned}
$$

Sendo que $b_{1} \notin A_{a_{1}}$, temos que $\left(a_{1}, b_{1}\right) \in \mathcal{P}(\mathcal{B})$. Como

$$
\begin{aligned}
b_{1}^{2} & =b^{2}-2 \rho_{1} \gamma_{4} a^{3} b+2\left(-\rho_{1} \gamma_{5}+\rho_{1}^{2} \gamma_{4}+\rho_{2} \gamma_{4}\right) a^{4} b+\rho_{1}^{2} \gamma_{4}^{2} a^{6} \\
& =\gamma_{4}^{2} a^{4}+\left(\alpha_{5}+2 \rho_{1} \gamma_{4}^{2}\right) a^{5}+\left(\alpha_{6}+3 \rho_{1}^{2} \gamma_{4}^{2}+2 \rho_{2} \gamma_{4}^{2}\right) a^{6}
\end{aligned}
$$

e

$$
\begin{aligned}
a_{1}^{2} b_{1} & =b a^{2}-\rho \gamma_{3} a^{4}-\rho\left(\gamma_{4}-\rho \gamma_{3}\right) a^{5}+2 \rho a^{3} b-2 \rho^{2} \gamma_{3} a^{5}+\rho^{2} a^{4} b \\
& =\gamma_{3} a^{3}+\left(\gamma_{4}-3 \rho \gamma_{3}\right) a^{4}+\left(\gamma_{5}-3 \rho \gamma_{4}\right) a^{5},
\end{aligned}
$$


temos a matriz,

$\left[\begin{array}{ccccccc}1 & 0 & 0 & 0 & 0 & 0 & 0 \\ 2 \rho_{1} & 1 & 0 & 0 & 0 & 0 & 0 \\ 2 \rho_{2}+\rho_{1}^{2} & 3 \rho_{1} & 1 & 0 & 0 & \gamma_{4}^{2} & \gamma_{4} \\ 2 \rho_{1} \rho_{2} & 3\left(\rho_{2}+\rho_{1}^{2}\right) & 4 \rho_{1} & 1 & 0 & \alpha_{5}+2 \rho_{1} \gamma_{4}^{2} & \gamma_{5}-3 \rho_{1} \gamma_{4} \\ \rho_{2}^{2} & \rho_{1}\left(6 \rho_{2}+\rho_{1}^{2}\right) & 4 \rho_{2}+6 \rho_{1}^{2} & 5 \rho_{2} & 1 & \alpha_{6}+3 \gamma_{4}^{2}\left(\rho_{1}^{2}+2 \rho_{2}\right) & \gamma_{6}+\mu\end{array}\right]$

com $\mu=-3\left(\rho_{1} \gamma_{5}-\rho_{2} \gamma_{4}\right)$, que reduzida por linhas nos leva a matriz

$$
\left[\begin{array}{ccccccc}
1 & 0 & 0 & 0 & 0 & 0 & 0 \\
0 & 1 & 0 & 0 & 0 & 0 & 0 \\
0 & 0 & 1 & 0 & 0 & \alpha_{4}^{2} & \gamma_{4} \\
0 & 0 & 0 & 1 & 0 & \alpha_{5}-7 \rho_{1} \gamma_{4}^{2} & \gamma_{4} \\
0 & 0 & 0 & 0 & 1 & \alpha_{6}-2 \rho_{2} \gamma_{4}^{2}+7 \rho_{1}^{2} \gamma_{4}^{2}-5 \rho_{1} \alpha_{5} & \gamma_{6}-8 \rho_{1} \gamma_{5}-\rho_{2} \gamma_{4}+29 \rho_{1}^{2} \gamma_{4}
\end{array}\right]
$$

que nos da as constantes de estrutura de $\mathcal{B}$ em relação a base determinada pelo par $\left(a_{1}, b_{1}\right)$, demonstrando o lema.

Estudemos os casos $\gamma_{4}=1$ e $\gamma_{4}=0$ separadamente.

- Caso 1: $\left(\gamma_{4}=1\right.$.) Temos a álgebra $\mathcal{B}\left(\alpha_{5}, \alpha_{6}, 1, \gamma_{5}, \gamma_{6}\right)$, que pelo Lema 4.29 é isomorfa à álgebra

$\mathcal{B}\left(\alpha_{5}-2 \rho_{1}, \alpha_{6}-2 \rho_{2}+7 \rho_{1}^{2}-5 \rho_{1} \alpha_{5}, 1, \gamma_{5}-7 \rho_{1}, \gamma_{6}-8 \rho_{1} \gamma_{5}-\rho_{2}+29 \rho_{1}^{2}\right)$.

Se $\rho_{1}=\frac{\gamma_{5}}{7}$ e $\rho_{2}=\gamma_{6}-\frac{27 \gamma_{5}^{2}}{49}$, então obtemos a álgebra $\mathcal{B}\left(\alpha_{5}^{\prime}, \alpha_{6}^{\prime}, 1,0,0\right)$.

Se $\alpha_{5}^{\prime} \neq 0$, então fazendo $\lambda=\alpha_{5}^{\prime}$ e $\beta=\alpha_{5}^{\prime 2}$, no Lema 4.28 obtemos

$$
\mathcal{B}\left(\alpha_{5}, \alpha_{6}, 1, \gamma_{5}, \gamma_{6}\right) \cong \mathcal{B}\left(1, \alpha_{6}^{\prime}, 1,0,0\right)
$$

e se $\alpha_{5}^{\prime}=0$, temos a álgebra $\mathcal{B}\left(0, \alpha_{6}^{\prime}, 1,0,0\right)$.

- Caso 2: $\left(\gamma_{4}=0\right.$.) Temos a álgebra $\mathcal{B}\left(\alpha_{5}, \alpha_{6}, 0, \gamma_{5}, \gamma_{5}\right)$, que pelo Lema 4.29 , é isomorfa à álgebra $\mathcal{B}\left(\alpha_{5}, \alpha_{6}-2 \rho_{1} \alpha_{5}, 0, \gamma_{5}, \gamma_{6}-8 \rho_{1} \gamma_{5}\right)$, para todo $\rho_{1}$ em $F$. 
- Se $\gamma_{5} \neq 0$, então considerando $\rho_{1}=\frac{\gamma_{6}}{8 \gamma_{5}}$, obtemos o isomorfismo com a álgebra $\mathcal{B}\left(\alpha_{5}, \alpha_{6}^{\prime}, 0, \gamma_{5}, 0\right)$, que é isomorfa a álgebra $\mathcal{B}\left(\alpha_{5}^{\prime}, \alpha_{6}^{\prime}, 0,1,0\right)$, quando tomamos $\beta=\gamma_{5}^{2}$ e $\lambda=\gamma_{5}$, no Lema 4.28 .

$*$ Se $\alpha_{5}^{\prime} \neq 0$, então fazendo $\lambda=\frac{1}{\alpha_{5}^{\prime}}$ e $\beta=\frac{1}{\alpha_{5}^{\prime 3}}$, no Lema 4.28 temos que

$$
\mathcal{B}\left(\alpha_{5}^{\prime}, \alpha_{6}^{\prime}, 0,1,0\right) \cong \mathcal{B}\left(1, \alpha_{6}^{\prime}, 0,1,0\right) .
$$

* Se $\alpha_{5}^{\prime}=0$, temos a álgebra $\mathcal{B}\left(0, \alpha_{6}^{\prime}, 0,1,0\right)$.

- Se $\gamma_{5}=0$, temos a álgebra $\mathcal{B}\left(\alpha_{5}, \alpha_{6}-5 \rho_{1} \alpha_{5}, 0,0, \gamma_{6}\right)$.

$*$ Se $\alpha_{5} \neq 0$, então fazendo $\rho_{1}=\frac{\alpha_{6}}{5 \alpha_{5}}$, obtemos o isomorfismo com a álgebra $\mathcal{B}\left(\alpha_{5}, 0,0,0, \gamma_{6}\right)$, e tomando $\lambda=\alpha_{5}$ e $\beta=\alpha_{5}^{2}$, no Lema 4.28 , obtemos que

$$
\mathcal{B}\left(\alpha_{5}, \alpha_{6}, 0,0, \gamma_{6}\right) \cong \mathcal{B}\left(1,0,0,0, \gamma_{6}\right) .
$$

* Se $\alpha_{5}=0$, temos a álgebra $\mathcal{B}\left(0, \alpha_{6}, 0,0, \gamma_{6}\right)$.

- Se $\gamma_{6} \neq 0$, então considerando $\lambda=\gamma_{6}$ e $\beta=\gamma_{6}^{3}$, no Lema 4.17,

$$
\mathcal{B}\left(0, \alpha_{6}, 0,0, \gamma_{6}\right) \cong \mathcal{B}\left(0, \alpha_{6}, 0,0,1\right) .
$$

- Se $\gamma_{6}=0$, temos a álgebra $\mathcal{B}\left(0, \alpha_{6}, 0,0,0\right)$.

Podemos concluir, de todo o anterior que, se $\mathcal{B}$ é uma nilálgebra comutativa de p.a. de dimensão e nilíndice 7 , então existe uma base da forma $\left\{b, a, a^{2}, a^{3}, a^{4}, a^{5}, a^{6}\right\}$, com produtos não triviais não zero, dados por um da seguinte lista, $\operatorname{com} \alpha$ em $F$.

$$
\begin{aligned}
& \mathcal{B}_{1}(\alpha): \quad b^{2}=a^{4}+a^{5}+\alpha a^{6}, \quad a^{2} b=a^{4}, \quad a^{3} b=-a^{5}, \quad a^{4} b=a^{6} ; \\
& \mathcal{B}_{2}(\alpha): \quad b^{2}=a^{4}+\alpha a^{6}, \quad a^{2} b=a^{4}, \quad a^{3} b=-a^{5}, \quad a^{4} b=a^{6} ; \\
& \mathcal{B}_{3}(\alpha): \quad b^{2}=a^{5}+\alpha a^{6}, \quad a^{2} b=a^{5}, \quad a^{3} b=-a^{6} ; \\
& \mathcal{B}_{4}(\alpha): \quad b^{2}=\alpha a^{6}, \quad a^{2} b=a^{5}, \quad a^{3} b=-a^{6} ;
\end{aligned}
$$




$$
\begin{array}{ll}
\mathcal{B}_{5}(\alpha): \quad b^{2}=a^{5}, \quad a^{2} b=\alpha a^{6} ; & \\
\mathcal{B}_{6}(\alpha): \quad b^{2}=\alpha a^{6}, \quad a^{2} b=a^{6} ; & \\
\mathcal{B}_{7}(\alpha): \quad b^{2}=\alpha a^{6}, & \alpha \neq 0 ;
\end{array}
$$

$\mathcal{B}_{8}:$

Iniciemos a analise das condições de isomorfismo. Igual que para dimensão cinco e seis, $\mathcal{B}_{8}$ não é isomorfa as outras sete álgebras por causa da dimensão do seu anulador. Se $\overline{\mathcal{B}}_{i}=\mathcal{B}_{i} / \operatorname{Anul}\left(\mathcal{B}_{i}\right)$, então calculando a dimensão do anulador das restantes $\overline{\mathcal{B}}_{i}$, separamos as álgebras $\mathcal{B}_{6}$ e $\mathcal{B}_{7}$ das cinco primeiras da lista. Finalmente calculando a dimensão do anulador do $\operatorname{Anul}\left(\overline{\mathcal{B}}_{i}\right)$, das cinco primeiras $\overline{\mathcal{B}}_{i}$, separamos $\mathcal{B}_{1}$ e $\mathcal{B}_{2}$ das três restantes. Assim as sete primeiras álgebras estão separadas em três conjuntos de álgebras não isomorfas, eles são

$$
C 1: \mathcal{B}_{1}, \mathcal{B}_{2} . \quad C 2: \mathcal{B}_{3}, \mathcal{B}_{4}, \mathcal{B}_{5} . \quad C 3: \mathcal{B}_{6}, \mathcal{B}_{7}
$$

Observamos que $\overline{\mathcal{B}}_{3}$ corresponde a $\mathcal{A}_{8}, \overline{\mathcal{B}}_{4}$ corresponde a $\mathcal{A}_{10}$ e $\overline{\mathcal{B}}_{5}$ corresponde a $\mathcal{A}_{6}$, do Teorema 4.26 , que não são isomorfas. Isto mostra que as álgebras de $C 2$ não são isomorfas. Resta então analisar os conjuntos $C 1$ e $C 3$.

Vejamos que as álgebras de $C 1$, são isomorfas.

Lema 4.30 Para todo $\alpha$ em F,

$$
\mathcal{B}_{2}(\alpha) \cong \mathcal{B}_{1}\left(\alpha-\frac{1}{16}\right)
$$

Prova: Sejam $\alpha$ em $F$ e $(a, b)$ em $\mathcal{P}\left(\mathcal{B}_{2}(\alpha)\right)$, tal que $b^{2}=a^{4}+\alpha a^{6}, a^{2} b=$ $a^{4}, a^{3} b=-a^{5}, a^{4} b=a^{6}$ e os outros produtos não triviais são zero. Se definimos

$$
a_{1}:=\frac{7}{16} b+a-\frac{1}{16} a^{2}-\frac{19}{64} a^{3} \quad \text { e } b_{1}:=b-\frac{3}{8} a^{3}-\frac{31}{64} a^{4}-\left(\frac{7}{16} \alpha-\frac{9}{128}\right) a^{5},
$$


então

$$
\begin{aligned}
& a_{1}^{2}=a^{2}-\frac{1}{8} a^{3}-\frac{29}{64} a^{4}+\frac{19}{64} a^{5}+\left(\frac{49}{256} \alpha+\frac{361}{498}\right) a^{6}, \\
& a_{1}^{3}=a^{3}+\frac{1}{4} a^{4}-\frac{11}{16} a^{5}+\frac{21}{128} a^{6}, \\
& a_{1}^{4}=a^{4}-\frac{1}{4} a^{5}-\frac{57}{64} a^{6}, \\
& a_{1}^{5}=a^{5}+\frac{1}{8} a^{6}, \\
& a_{1}^{6}=a^{6} \neq 0,
\end{aligned}
$$

e

$$
\begin{aligned}
a_{1} b_{1}= & \frac{7}{16} b^{2}-\frac{21}{128} a^{3} b-\frac{217}{1024} a^{4} b-\left(\frac{3}{8} a^{4}-\frac{31}{64}\right) a^{5}-\left(\frac{7}{16} \alpha-\frac{9}{128}\right) a^{6}- \\
& \frac{1}{16} a^{2} b+\frac{3}{128} a^{5}+\frac{31}{1024} a^{6}-\frac{19}{64} a^{3} b+\frac{57}{512} a^{6} \\
= & \left(\frac{7}{16}-\frac{3}{8}-\frac{1}{16}\right) a^{4}+\left(\frac{21}{128}-\frac{31}{64}+\frac{3}{128}+\frac{19}{64}\right) a^{5}+ \\
& \left(\frac{7}{16} \alpha-\frac{217}{1024}-\frac{7}{16} \alpha+\frac{9}{128}+\frac{31}{1024}+\frac{57}{512}\right) a^{6} \\
= & 0 .
\end{aligned}
$$

O anterior somado ao fato de $b_{1} \notin A_{a_{1}}$ mostra que $\left(a_{1}, b_{1}\right) \in \mathcal{P}\left(\mathcal{B}_{2}(\alpha)\right)$, e uma vez que

$$
\begin{aligned}
b_{1}^{2} & =b^{2}-\frac{3}{4} a^{3} b-\frac{31}{32} a^{4} b+\frac{9}{64} a^{6}=a^{4}+\alpha a^{6}+\frac{3}{4} a^{5}-\frac{31}{32} a^{6}+\frac{9}{64} a^{6} \\
& =a^{4}+\frac{3}{4} a^{5}+\left(\alpha-\frac{53}{64}\right) \\
& =\left(a_{1}^{4}+\frac{1}{4} a_{1}^{5}+\frac{55}{64} a_{1}^{6}\right)+\left(a_{1}^{5}-\frac{1}{8} a_{1}^{6}\right)+\left(\alpha-\frac{53}{64}\right) a_{1}^{6} \\
& =a_{1}^{4}+a_{1}^{5}+\left(\alpha-\frac{1}{16}\right) a_{1}^{6}
\end{aligned}
$$


e

$$
\begin{aligned}
a_{1}^{2} b_{1} & =b a^{2}-\frac{1}{8} a^{3} b-\frac{29}{64} a^{4} b+\frac{3}{64} a^{6}-\frac{31}{64} a^{6} \\
& =a^{4}+\frac{1}{8} a^{5}-\frac{29}{64} a^{6}+\frac{3}{64} a^{6}-\frac{31}{64} a^{6} \\
& =a^{4}-\frac{1}{4} a^{5}-\frac{57}{64} a^{6} \\
& =\left(a_{1}^{4}+\frac{1}{4} a_{1}^{5}+\frac{55}{64} a_{1}^{6}\right)-\frac{1}{4}\left(a_{1}^{5}-\frac{1}{8} a_{1}^{6}\right)-\frac{57}{64} a_{1}^{6} \\
& =a_{1}^{4},
\end{aligned}
$$

temos o isomorfismo.

Temos então que a classe determinada por $\mathcal{B}_{1}(\alpha)$ está contida na classe determinada por $\mathcal{B}_{2}(\alpha)$. Assim

$$
\mathcal{B}_{1} \nsucceq \mathcal{B}_{i} \text { para todo } i \neq 1,2 \text {. }
$$

Para finalizar o análise das condições de isomorfismo, resta demonstrar que as álgebras do conjunto $C 2$ não são isomorfas, como concluiremos do seguinte lema.

Lema 4.31 Seja $\alpha$ em $F^{*}$. Para cada par $\left(a_{1}, b_{1}\right)$ em $\mathcal{P}\left(\mathcal{B}_{7}(\alpha)\right)$,

$$
b_{1}^{2}=\rho^{2} \alpha a_{1}^{6} \quad \text { e } a_{1}^{2} b_{1}=0,
$$

com $\rho$ não nulo e os outros produtos não triviais são zero.

Prova: Seja $\alpha$ não nulo e $\left\{b, a, a^{2}, a^{3}, a^{4}, a^{5}, a^{6}\right\}$, uma base para $\mathcal{B}_{7}(\alpha)$, tal que $b^{2}=\alpha a^{6}$ e os outros produtos não triviais são zero. Então se $\left(a_{1}, b_{1}\right)$ é um elemento de $\mathcal{P}\left(\mathcal{B}_{7}(\alpha)\right)$, $a_{1}$ e $b_{1}$ são da forma $a_{1}=\lambda b+\lambda_{1} a+\lambda_{2} a^{2}+$ $\lambda_{3} a^{3}+\lambda_{4} a^{4}+\lambda_{5} a^{5}+\lambda_{6} a^{6}$ e $b_{1}=\beta b+\beta_{1} a+\beta_{2} a^{2}+\beta_{3} a^{3}+\beta_{4} a^{4}+\beta_{5} a^{5}+\beta_{6} a^{6}$. Utilizando a equação (4.11), para $\gamma_{4}=\gamma_{5}=\gamma_{6}=0$ e $\alpha_{6}=\alpha$, temos que as potências de $a_{1}$ são dadas por 


$$
\begin{aligned}
a_{1}^{2}= & \lambda_{1}^{2} a^{2}+2 \lambda_{1} \lambda_{2} a^{3}+\left(2 \lambda_{1} \lambda_{3}+\lambda_{2}^{2}\right) a^{4}+\left(2 \lambda_{1} \lambda_{4}+2 \lambda_{2} \lambda_{3}\right) a^{5}+\left(\lambda^{2} \alpha+\right. \\
& \left.2 \lambda_{1} \lambda_{5}+2 \lambda_{2} \lambda_{4}+\lambda_{3}^{2}\right) a^{6}, \\
a_{1}^{3}= & \lambda_{1}^{3} a^{3}+3 \lambda_{1}^{2} \lambda_{2} a^{4}+3\left(\lambda_{3} \lambda_{1}^{2}+\lambda_{2}^{2} \lambda_{1}\right) a^{5}+\left(3 \lambda_{4} \lambda_{1}^{2}+6 \lambda_{1} \lambda_{2} \lambda_{3}+\lambda_{2}^{3}\right) a^{6} \\
a_{1}^{4}= & \lambda_{1}^{4} a^{4}+4 \lambda_{1}^{3} \lambda_{2} a^{5}+\left(4 \lambda_{1}^{3} \lambda_{3}+6 \lambda_{2}^{2} \lambda_{1}^{2}\right) a^{6} \\
a_{1}^{5}= & \lambda_{1}^{5} a^{5}+5 \lambda_{1}^{4} \lambda_{2} a^{6} \\
a_{1}^{6}= & \lambda_{1}^{6} a^{6} \neq 0
\end{aligned}
$$

$\operatorname{logo} \lambda_{1} \neq 0$. Temos também que,

$$
\begin{aligned}
a_{1} b_{1}= & \lambda \beta b^{2}+\lambda_{1} \beta_{1} a^{2}+\lambda_{1} \beta_{2} a^{3}+\lambda_{1} \beta_{3} a^{4}+\lambda_{1} \beta_{4} a^{5}+\lambda_{1} \beta_{5} a^{6}+\lambda_{2} \beta_{1} a^{3}+ \\
& \lambda_{2} \beta_{2} a^{4}+\lambda_{2} \beta_{3} a^{5}+\lambda_{2} \beta_{4} a^{6}+\lambda_{3} \beta_{1} a^{4}+\lambda_{3} \beta_{2} a^{5}++\lambda_{3} \beta_{3} a^{6} \\
& +\lambda_{4} \beta_{1} a^{5}+\lambda_{4} \beta_{2} a^{6}+\lambda_{5} \beta_{1} a^{6}+\lambda_{3}^{2} a^{6} \\
= & \lambda_{1} \beta_{1} a^{2}+\left(\lambda_{1} \beta_{2}+\lambda_{2} \beta_{1}\right) a^{3}+\left(\lambda_{1} \beta_{3}+\lambda_{2} \beta_{2}+\lambda_{3} \beta_{1}\right) a^{4} \\
& +\left(\lambda_{1} \beta_{4}+\lambda_{3} \beta_{2}+\lambda_{4} \beta_{1}+\lambda_{2} \beta_{3}\right) a^{5}+\left(\lambda \beta \alpha+\lambda_{1} \beta_{5}+\lambda_{5} \beta_{1}+\lambda_{2} \beta_{4}\right. \\
& \left.+\lambda_{4} \beta_{2}+\lambda_{3} \beta_{3}\right) a^{6} \\
= & 0,
\end{aligned}
$$

$\operatorname{logo} \lambda_{1} \beta_{1}=0$ e como $\lambda_{1} \neq 0$, segue que $\beta_{1}=0$, igualmente $\beta_{2}=\beta_{3}=$ $\beta_{4}=0$. Portanto $\beta_{5}=-\frac{\lambda \beta \alpha}{\lambda_{1}}$, e assim

$$
b_{1}=\beta b-\frac{\beta \lambda \alpha}{\lambda_{1}} a^{5}+\beta_{6} a^{6}, \quad \operatorname{com} \quad \beta \neq 0 .
$$

Logo

$$
b_{1}^{2}=\beta^{2} b^{2}=\beta^{2} \alpha a^{6}=\frac{\beta^{2}}{\lambda_{1}^{6}} \alpha a_{1}^{6} \quad \text { e } \quad a_{1}^{2} b_{1}=0, \quad \text { com } \quad \lambda_{1}, \beta \neq 0 .
$$

Corolário 4.32 Sejam $\alpha$ e $\alpha^{\prime}$ não nulos. Então

$$
\mathcal{B}_{7}(\alpha) \cong \mathcal{B}_{7}\left(\alpha^{\prime}\right)
$$

se, e somente se, existir $\rho$ não nulo tal que $\alpha^{\prime}=\rho^{2} \alpha$. 
Como uma consequência do lema anterior temos que para $\alpha$ não nulo e $\alpha^{\prime}$ em $F$,

$$
\mathcal{B}_{6}\left(\alpha^{\prime}\right) ¥ \mathcal{B}_{7}(\alpha)
$$

Podemos concluir portanto que

$$
\mathcal{B}_{i} \not \mathcal{B}_{j} \text { para todo } i \neq j \text {. }
$$

Estudaremos agora as classes dadas por cada $\mathcal{B}_{i}(\alpha)$ para um $\alpha$ qualquer. Uma vez que a classe dada por $\mathcal{B}_{7}(\alpha)$ já foi estudada e que a classe dada por $\mathcal{B}_{1}(\alpha)$ está contida na classe dada por $\mathcal{B}_{2}(\alpha)$, resta analisar as classes dadas para $i=2,3,4,5,6$.

Lema 4.33 Sejam $\alpha$ e $\alpha^{\prime}$ em F. Então

$$
\mathcal{B}_{6}(\alpha) \cong \mathcal{B}_{6}\left(\alpha^{\prime}\right)
$$

se, e somente se, existir um $\rho$ em $F^{*}$ tal que $\alpha^{\prime}=\rho^{2} \alpha$.

Prova: Sejam $\alpha$ e $\alpha^{\prime}$ em $F$ e $(a, b)$ em $\mathcal{P}\left(\mathcal{B}_{6}(\alpha)\right)$ tal que $b^{2}=\alpha a^{6}$ e $a^{2} b=a^{6}$. Sejam $\left(a_{1}, b_{1}\right) \in \mathcal{P}\left(\mathcal{B}_{6}(\alpha)\right)$. Vejamos como são os produtos $b_{1}^{2}$ e $a_{1}^{2} b_{1}$. O par $\left(a_{1}, b_{1}\right)$ exprime-se na base determinada pelo par $(a, b)$, como $a_{1}=\lambda b+\lambda_{1} a+$ $\lambda_{2} a^{2}+\lambda_{3} a^{3}+\lambda_{4} a^{4}+\lambda_{5} a^{5}+\lambda_{6} a^{6}$ e $b_{1}=\beta b+\beta_{1} a+\beta_{2} a^{2}+\beta_{3} a^{3}+\beta_{4} a^{4}+\beta_{5} a^{5}+\lambda_{6} a^{6}$. Por (4.11), para $\alpha_{4}=\gamma_{4}=\gamma_{5}=0, \gamma_{6}=1$ e $\alpha_{6}=\alpha$, temos as potências de $a_{1}$ dadas por

$$
\begin{aligned}
a_{1}^{2}= & \lambda_{1}^{2} a^{2}+2 \lambda_{1} \lambda_{2} a^{3}+\left(2 \lambda_{1} \lambda_{3}+\lambda_{2}^{2}\right) a^{4}+\left(2 \lambda_{1} \lambda_{4}+2 \lambda_{2} \lambda_{3}\right) a^{5}+\left(\lambda^{2} \alpha+\right. \\
& \left.2 \lambda \lambda_{2}+2 \lambda_{1} \lambda_{5}+2 \lambda_{2} \lambda_{4}+\lambda_{3}^{2}\right) a^{6} \\
a_{1}^{3}= & \lambda_{1}^{3} a^{3}+3 \lambda_{1}^{2} \lambda_{2} a^{4}+3\left(\lambda_{3} \lambda_{1}^{2}+\lambda_{2}^{2}\right) a^{5}+\left(3 \lambda_{4} \lambda_{1}^{2}+6 \lambda_{1} \lambda_{2} \lambda_{3}+\lambda_{2}^{3}\right) a^{6}, \\
a_{1}^{4}= & \lambda_{1}^{4} a^{4}+4 \lambda_{1}^{3} \lambda_{2} a^{5}+\left(4 \lambda_{1}^{3} \lambda_{3}+6 \lambda_{2}^{2} \lambda_{1}^{2}\right) a^{6}, \\
a_{1}^{5}= & \lambda_{1}^{5} a^{5}+4 \lambda_{1}^{4} \lambda_{2} a^{6} \\
a_{1}^{6}= & \lambda_{1}^{6} a^{6} \neq 0 .
\end{aligned}
$$


Agora,

$$
\begin{aligned}
a_{1} b_{1}= & \lambda \beta b^{2}+\lambda \beta_{2} a^{2} b+\lambda_{1} \beta_{1} a^{2}+\lambda_{1} \beta_{2} a^{3}+\lambda_{1} \beta_{3} a^{4}+\lambda_{1} \beta_{4} a^{5}+\lambda_{1} \beta_{5} a^{6}+ \\
& \lambda_{2} \beta a^{2} b+\lambda_{2} \beta_{1} a^{3}+\lambda_{2} \beta_{2} a^{4}+\lambda_{2} \beta_{3} a^{5}+\lambda_{2} \beta_{4} a^{6}+\lambda_{3} \beta_{1} a^{4}+\lambda_{3} \beta_{2} a^{5}+ \\
& \lambda_{3} \beta_{3} a^{6}+\lambda_{4} \beta_{1} a^{5}+\lambda_{4} \beta_{2} a^{6}+\lambda_{5} \beta_{1} a^{6}+\lambda_{3}^{2} a^{6} \\
= & \lambda_{1} \beta_{1} a^{2}+\left(\lambda_{1} \beta_{2}+\lambda_{2} \beta_{1}\right) a^{3}+\left(\lambda_{1} \beta_{3}+\lambda_{2} \beta_{2}+\lambda_{3} \beta_{1}\right) a^{4}+\left(\lambda_{1} \beta_{4}+\lambda_{3} \beta_{2}\right. \\
& \left.+\lambda_{4} \beta_{1}+\lambda_{2} \beta_{3}\right) a^{5}+\left(\lambda \beta \alpha+\lambda \beta_{2} a^{2} b+\lambda_{2} \beta a^{2} b+\lambda_{1} \beta_{5}+\lambda_{5} \beta_{1}+\lambda_{2} \beta_{4}\right. \\
& \left.+\lambda_{4} \beta_{2}+\lambda_{3} \beta_{3}\right) a^{6} \\
= & 0,
\end{aligned}
$$

$\operatorname{logo} \lambda_{1} \beta_{1}=0$ e dado que $\lambda_{1} \neq 0$, segue que $\beta_{1}=0$, igualmente $\beta_{2}=\beta_{3}=$ $\beta_{4}=0$ e portanto $\beta_{5}=\frac{-\lambda \beta \alpha+\lambda_{2} \beta}{\lambda_{1}}$. Assim

$$
b_{1}=\beta b-\frac{\lambda \beta \alpha+\lambda_{2} \beta}{\lambda_{1}} a^{5}+\lambda_{1} \beta_{6} a^{6}, \quad \text { com } \beta \neq 0 .
$$

Portanto

$$
b_{1}^{2}=\beta^{2} b^{2}=\beta^{2} \alpha a^{6}=\frac{\beta^{2}}{\lambda_{1}^{6}} \alpha a_{1}^{6} \quad \text { e } \quad a_{1}^{2} b_{1}=\alpha \beta a^{2} b=\lambda_{1}^{2} \beta a^{5}=\frac{\beta}{\lambda_{1}^{4}} a_{1}^{5},
$$

com $\lambda_{1}, \beta \neq 0$. Logo para um $\alpha^{\prime}$ em $F$, temos que $\mathcal{B}_{6}(\alpha) \cong \mathcal{B}_{6}\left(\alpha^{\prime}\right)$, se e somente se,

$$
\alpha^{\prime}=\frac{\beta^{2}}{\lambda_{1}^{6}} \alpha a_{1}^{6} \quad \text { e } \quad \frac{\beta}{\lambda_{1}^{4}}=1,
$$

de onde $\beta=\lambda_{1}^{4}$ e portanto $\alpha^{\prime}=\frac{1}{\lambda_{1}^{2}} \alpha$, o que demonstra o lema.

Lema 4.34 Sejam $\alpha$ e $\alpha^{\prime}$ em F. Então

$$
\mathcal{B}_{5}(\alpha) \cong \mathcal{B}_{5}\left(\alpha^{\prime}\right)
$$

se, e somente se, existir um $\rho$ não nulo tal que $\alpha^{\prime}=\rho^{2} \alpha$.

Prova: Seja $\left\{b, a, a^{2}, a^{3}, a^{4}, a^{5}\right\}$ uma base para $\mathcal{B}_{5}(\alpha)$ com produtos dados por $b^{2}=a^{5}$ e $a^{2} b=\alpha a^{6}$, para $\alpha$ em $F$. Se $\left(a_{1}, b_{1}\right) \in \mathcal{P}\left(\mathcal{B}_{6}(\alpha)\right)$, então 


$$
\begin{aligned}
a_{1}= & \lambda b+\lambda_{1} a+\lambda_{2} a^{2}+\lambda_{3} a^{3}+\lambda_{4} a^{4}+\lambda_{5} a^{5}+\lambda_{6} a^{6} \text { e } b_{1}=\beta b+\beta_{1} a+\beta_{2} a^{2}+ \\
\beta_{3} a^{3}+ & \beta_{4} a^{4}+\beta_{5} a^{5}+\lambda_{6} a^{6} . \text { Por }(4.11), \text { as potências de } a_{1} \text { são dadas por } \\
a_{1}^{2}= & \lambda_{1}^{2} a^{2}+2 \lambda_{1} \lambda_{2} a^{3}+\left(2 \lambda_{1} \lambda_{3}+\lambda_{2}^{2}\right) a^{4}+\left(\lambda^{2}+2 \lambda_{1} \lambda_{4}+2 \lambda_{2} \lambda_{3}\right) a^{5}+\left(2 \lambda \lambda_{2} \alpha+\right. \\
& \left.2 \lambda_{1} \lambda_{5}+2 \lambda_{2} \lambda_{4}+\lambda_{3}^{2}\right) a^{6}, \\
a_{1}^{3}= & \lambda_{1}^{3} a^{3}+3 \lambda_{1}^{2} \lambda_{2} a^{4}+3\left(\lambda_{3} \lambda_{1}^{2}+\lambda_{2}^{2} \lambda_{1}\right) a^{5}+\left(\lambda \lambda_{1}^{2} \alpha+\lambda_{1} \lambda^{2}+3 \lambda_{4} \lambda_{1}^{2}+6 \lambda_{1} \lambda_{2} \lambda_{3}\right. \\
& \left.+\lambda_{2}^{3}\right) a^{6}, \\
a_{1}^{4}= & \lambda_{1}^{4} a^{4}+4 \lambda_{1}^{3} \lambda_{2} a^{5}+\left(4 \lambda_{1}^{3} \lambda_{3}+6 \lambda_{2}^{2} \lambda_{1}^{2}\right) a^{6}, \\
a_{1}^{5}= & \lambda_{1}^{5} a^{5}+5 \lambda_{1}^{4} \lambda_{2} a^{6}, \\
a_{1}^{6}= & \lambda_{1}^{6} a^{6} \neq 0,
\end{aligned}
$$

$\operatorname{logo} \lambda_{1} \neq 0$, pois $a_{1}$ tem nilíndice máximo. Como o produto de $a_{1}$ com $b_{1}$ é nulo, temos que

$$
\begin{aligned}
a_{1} b_{1}= & \lambda \beta b^{2}+\lambda \beta_{2} a^{2} b+\lambda_{1} \beta_{1} a^{2}+\lambda_{1} \beta_{2} a^{3}+\lambda_{1} \beta_{3} a^{4}+\lambda_{1} \beta_{4} a^{5}+\lambda_{1} \beta_{5} a^{6}+ \\
& \lambda_{2} \beta a^{2} b+\lambda_{2} \beta_{1} a^{3}+\lambda_{2} \beta_{2} a^{4}+\lambda_{2} \beta_{3} a^{5}+\lambda_{2} \beta_{4} a^{6}+\lambda_{3} \beta_{1} a^{4}+\lambda_{3} \beta_{2} a^{5}+ \\
& \lambda_{3} \beta_{3} a^{6}+\lambda_{4} \beta_{1} a^{5}+\lambda_{4} \beta_{2} a^{6}+\lambda_{5} \beta_{1} a^{6}+\lambda_{3}^{2} a^{6} \\
= & \lambda_{1} \beta_{1} a^{2}+\left(\lambda_{1} \beta_{2}+\lambda_{2} \beta_{1}\right) a^{3}+\left(\lambda_{1} \beta_{3}+\lambda_{2} \beta_{2}+\lambda_{3} \beta_{1}\right) a^{4}+\left(\lambda \beta+\lambda_{1} \beta_{4}\right. \\
& \left.+\lambda_{3} \beta_{2}+\lambda_{4} \beta_{1}+\lambda_{2} \beta_{3}\right) a^{5}+\left(\lambda \beta_{2} \alpha+\lambda_{2} \beta \alpha+\lambda_{1} \beta_{5}+\lambda_{5} \beta_{1}+\lambda_{2} \beta_{4}\right. \\
& \left.+\lambda_{4} \beta_{2}+\lambda_{3} \beta_{3}\right) a^{6} \\
= & 0,
\end{aligned}
$$

$\log \mathrm{o} \beta_{1}=\beta_{2}=\beta_{3}=0$, pois $\lambda_{1} \neq 0$ e então $\beta_{4}=-\frac{\lambda \beta}{\lambda_{1}}$ e $\beta_{5}=-\frac{\beta \lambda_{2}\left(\lambda_{1} \alpha-\lambda\right)}{\lambda_{1}^{2}}$. Assim

$$
b_{1}=\beta b-\frac{\lambda \beta}{\lambda_{1}} a^{4}-\frac{\beta \lambda_{2}\left(\lambda_{1} \alpha-\lambda\right)}{\lambda_{1}^{2}} a^{5}+\beta_{6} a^{6}, \quad \text { com } \quad \beta \neq 0 .
$$

Portanto

$$
b_{1}^{2}=\beta^{2} b^{2}=\beta^{2} a^{5}=\frac{\beta^{2}}{\lambda_{1}^{6}} \alpha a_{1}^{6} \quad \text { e } \quad a_{1}^{2} b_{1}=\alpha \beta a^{2} b=\lambda_{1}^{2} \beta a^{5}=\frac{\beta}{\lambda_{1}^{4}} a_{1}^{5},
$$

com $\lambda_{1}, \beta \neq 0$. Logo para um $\alpha^{\prime}$ em $F$, temos que $\mathcal{B}_{6}(\alpha) \cong \mathcal{B}_{6}\left(\alpha^{\prime}\right)$, se e somente se,

$$
\alpha^{\prime}=\frac{\beta^{2}}{\lambda_{1}^{6}} \alpha a_{1}^{6} \quad \text { e } \quad \frac{\beta}{\lambda_{1}^{4}}=1
$$


de onde $\beta=\lambda_{1}^{4}$ e portanto $\alpha^{\prime}=\frac{1}{\lambda_{1}^{2}} \alpha$, o que demonstra o lema.

O estudo das classes determinadas por $\mathcal{B}_{3}$ e $\mathcal{B}_{4}$ será feito conjuntamente no seguinte lema.

Lema 4.35 Seja $\mathcal{B}$ uma nilálgebra comutativa de p.a. de dimensão e nilíndice 7, com base $\left\{b, a, a^{2}, a^{3}, a^{4}, a^{5}, a^{6}\right\}$ tal que $b^{2}=\theta_{1} a^{5}+\theta_{2} a^{6}, a^{2} b=a^{5} e$ $a^{3} b=-a^{6}$ e os outros produtos não triviais são zero. Então para cada par $\left(a_{1}, b_{1}\right)$ em $\mathcal{P}(\mathcal{B})$, existem $\lambda_{1}, \beta \in F^{*}$ e $\lambda_{2}, \lambda \in F$, tais que

$$
b_{1}^{2}=\frac{\beta^{2}}{\lambda_{1}^{5}} \theta_{1} a_{1}^{5}+\frac{\beta^{2}}{\lambda_{1}^{7}}\left(\theta_{2} \lambda_{1}-5 \theta_{1} \lambda_{2}\right) a_{1}^{6} \quad e \quad a_{1}^{2} b_{1}=\frac{\beta}{\lambda_{1}^{3}} a_{1}^{5}-\frac{\beta}{\lambda_{1}^{5}}\left(8 \lambda_{2}-\theta_{1} \lambda\right) a_{1}^{6} .
$$

Prova: Seja $\mathcal{B}$ como no enunciado do lema e $\left(a_{1}, b_{1}\right) \in \mathcal{P}(\mathcal{B})$. Então $a_{1}$ e $b_{1}$ exprimem-se de maneira única na forma, $a_{1}=\lambda b+\lambda_{1} a+\lambda_{2} a^{2}+\lambda_{3} a^{3}+$ $\lambda_{4} a^{4}+\lambda_{5} a^{5}+\lambda_{6} a^{6}$ e $b_{1}=\beta b+\beta_{1} a+\beta_{2} a^{2}+\beta_{3} a^{3}+\beta_{4} a^{4}+\beta_{5} a^{5}+\beta_{6} a^{6}$. As potências de $a_{1}$, dadas por (4.11), são

$$
\begin{aligned}
a_{1}^{2}= & \lambda_{1}^{2} a^{2}+2 \lambda_{1} \lambda_{2} a^{3}+\left(\lambda^{2} \gamma_{4}^{2}+2 \lambda_{1} \lambda_{3}+\lambda_{2}^{2}\right) a^{4}+\left(\lambda^{2} \theta_{1}+2 \lambda \lambda_{2}+2 \lambda_{1} \lambda_{4}\right. \\
& \left.+2 \lambda_{2} \lambda_{3}\right) a^{5}+\left(\lambda^{2} \theta_{2}-2 \lambda \lambda_{3}+2 \lambda_{1} \lambda_{5}+2 \lambda_{2} \lambda_{4}+\lambda_{3}^{2}\right) a^{6}, \\
a_{1}^{3}= & \lambda_{1}^{3} a^{3}+3 \lambda_{1}^{2} \lambda_{2} a^{4}+\left(\lambda \lambda_{1}^{2}+3 \lambda_{3} \lambda_{1}^{2}+3 \lambda_{1} \lambda_{2}^{2}\right) a^{5}+\left(\lambda_{1} \lambda^{2} \theta_{1}+3 \lambda_{1}^{2} \lambda_{4}+\right. \\
& \left.6 \lambda_{1} \lambda_{2} \lambda_{3}+\lambda_{2}^{3}\right) a^{6} \\
a_{1}^{4}= & \lambda_{1}^{4} a^{4}+4 \lambda_{1}^{3} \lambda_{2} a^{5}+\left(4 \lambda_{1}^{3} \lambda_{3}+6 \lambda_{1}^{2} \lambda_{2}^{2}\right) a^{6}, \\
a_{1}^{5}= & \lambda_{1}^{5} a^{5}+5 \lambda_{1}^{4} \lambda_{2} a^{6} \\
a_{1}^{6}= & \lambda_{1}^{6} a^{6}
\end{aligned}
$$

e sendo que $a_{1}$ tem nilíndice máximo, temos que $\lambda_{1} \neq 0$. Por outro lado

$$
\begin{aligned}
a_{1} b_{1}= & \lambda \beta b^{2}+\lambda \beta_{2} a^{2} b+\lambda \beta_{3} a^{3} b+\lambda_{1} \beta_{1} a^{2}+\lambda_{1} \beta_{2} a^{3}+\lambda_{1} \beta_{3} a^{4}+\lambda_{1} \beta_{4} a^{5} \\
& +\lambda_{1} \beta_{5} a^{6}+\lambda_{2} \beta a^{2} b+\lambda_{2} \beta_{1} a^{3}+\lambda_{2} \beta_{2} a^{4}+\lambda_{2} \beta_{3} a^{5}+\lambda_{2} \beta_{4} a^{6} \\
& +\lambda_{3} \beta a^{3} b+\lambda_{3} \beta_{1} a^{4}+\lambda_{3} \beta_{2} a^{5}+\lambda_{3} \beta_{3} a^{6}+\lambda_{4} \beta_{1} a^{5}+\lambda_{4} \beta_{2} a^{6}
\end{aligned}
$$




$$
\begin{aligned}
= & \lambda_{1} \beta_{1} a^{2}+\left(\lambda_{1} \beta_{2}+\lambda_{2} \beta_{1}\right) a^{3}+\left(\lambda_{1} \beta_{3}+\lambda_{2} \beta_{2}+\lambda_{3} \beta_{1}\right) a^{4} \\
& +\left(\lambda \beta \theta_{1}+\lambda \beta_{2}+\lambda_{1} \beta_{4}+\lambda_{2} \beta+\lambda_{2} \beta_{3}+\lambda_{3} \beta_{2}+\lambda_{4} \beta_{1}\right) a^{5} \\
& +\left(\lambda \beta \theta_{2}-\lambda \beta_{3}+\lambda_{1} \beta_{5}+\lambda_{2} \beta_{4}-\lambda_{3} \beta+\lambda_{3} \beta_{3}+\lambda_{4} \beta_{2}\right) a^{6} \\
= & 0,
\end{aligned}
$$

$\operatorname{logo} \lambda_{1} \beta_{1}=0$ e como $\lambda_{1} \neq 0$, segue que $\beta_{1}=0$. Igualmente $\beta_{2}=\beta_{3}=0$, e portanto $\beta_{4}=-\frac{\beta\left(\lambda \theta_{1}+\lambda_{2}\right)}{\lambda_{1}} \mathrm{e}$

$$
\beta_{5}=-\frac{\beta}{\lambda_{1}}\left(\lambda \lambda_{1} \theta_{2}+\lambda_{1}^{2}+\lambda_{2} \lambda \theta_{1}+\lambda_{2}^{2}-\lambda_{3} \lambda_{1}\right) .
$$

Assim

$$
b_{1}=\beta b-\frac{\beta\left(\lambda \theta_{1}+\lambda_{2}\right)}{\lambda_{1}} a^{4}-\frac{\beta}{\lambda_{1}}\left(\lambda \lambda_{1} \theta_{2}+\lambda_{1}^{2}+\lambda_{2} \lambda \theta_{1}+\lambda_{2}^{2}-\lambda_{3} \lambda_{1}\right) a^{5}+\beta_{6} a^{6},
$$

$\operatorname{com} \beta \neq 0$. Portanto

$$
\begin{aligned}
b_{1}^{2} & =\beta^{2} b^{2}=\beta^{2} \theta_{1} a^{5}+\beta^{2} \theta_{2} a^{6}=\beta^{2} \theta_{1}\left[\frac{1}{\lambda_{1}^{5}} a_{1}^{5}-5 \frac{\lambda_{2}}{\lambda_{1}^{7}} a_{1}^{6}\right]+\beta^{2} \theta_{2} \frac{1}{\lambda_{1}^{6}} a_{1}^{6} \\
& =\frac{\beta^{2}}{\lambda_{1}^{5}} \theta_{1} a_{1}^{5}+\frac{\beta^{2}\left(\theta_{2} \lambda_{1}-5 \theta_{1} \lambda_{2}\right)}{\lambda_{1}^{7}} a_{1}^{6},
\end{aligned}
$$

$\mathrm{e}$

$$
\begin{aligned}
a_{1}^{2} b_{1} & =\beta \lambda_{1}^{2} a^{2} b+2 \beta \lambda_{1} \lambda_{2} a^{3} b-\frac{\lambda_{1}^{2} \beta\left(\lambda \theta_{1}+\lambda_{2}\right)}{\lambda_{1}} a^{6}=\beta \lambda_{1}^{2} a^{5}-\beta \lambda_{1}\left(3 \lambda_{2}+\lambda \theta_{1}\right) a^{6} \\
& =\beta \lambda_{1}^{2}\left[\frac{1}{\lambda_{1}^{5}} a_{1}^{5}-5 \frac{\lambda_{2}}{\lambda_{1}^{7}} a_{1}^{6}\right]-\beta \lambda_{1}\left(3 \lambda_{2}+\lambda \theta_{1}\right) \frac{1}{\lambda_{1}^{6}} a_{1}^{6}=\frac{\beta}{\lambda_{1}^{3}} a_{1}^{5}-\frac{\beta}{\lambda_{1}^{5}}\left(8 \lambda_{2}-\theta_{1} \lambda\right) a_{1}^{6} .
\end{aligned}
$$

o que demonstra o lema.

Corolário 4.36 Sejam $\alpha$ em $\alpha^{\prime}$ em F. Então

$$
\mathcal{B}_{4}(\alpha) \cong \mathcal{B}_{4}\left(\alpha^{\prime}\right)
$$

se e somente se, $\alpha^{\prime}=\alpha$. 
Prova: Para $\theta_{1}=0$ e $\theta_{2}=\alpha$, no lema anterior, temos a álgebra $\mathcal{B}_{4}(\alpha)$, portanto para cada par $\left(a_{1}, b_{1}\right)$ em $\mathcal{P}\left(\mathcal{B}_{4}(\alpha)\right)$, cumpres-se que

$$
b_{1}^{2}=\frac{\beta^{2} \alpha}{\lambda_{1}^{6}} a_{1}^{6} \quad \text { e } \quad a_{1}^{2} b_{1}=\frac{\beta}{\lambda_{1}^{3}} a^{5}-\frac{8 \beta \lambda_{2}}{\lambda_{1}^{5}} \quad \text { com } \quad \lambda_{1}, \beta \neq 0 .
$$

Logo para $\alpha^{\prime}$ em $F, \mathcal{B}_{4}(\alpha)$ é isomorfa a $\mathcal{B}_{4}\left(\alpha^{\prime}\right)$, se e somente se $\alpha^{\prime}=\frac{\beta^{2} \alpha}{\lambda_{1}^{6}}, 1=$ $\frac{\beta}{\lambda_{1}^{3}}$ e $0=-\frac{8 \beta \lambda_{2}}{\lambda_{1}^{5}}$. Portanto $\lambda_{2}=0, \beta=\lambda_{1}^{3}$ e $\alpha^{\prime}=\frac{\lambda_{1}^{6} \alpha}{\lambda_{1}^{6}}=\alpha$.

Corolário 4.37 Para todo $\alpha$ e $\alpha^{\prime}$ em F,

$$
\mathcal{B}_{3}(\alpha) \cong \mathcal{B}_{3}\left(\alpha^{\prime}\right)
$$

Prova: Se $\theta_{1}=1$ e $\theta_{2}=\alpha$, no lema anterior, temos a álgebra $\mathcal{B}_{4}(\alpha)$, logo para todo par $\left(a_{1}, b_{1}\right) \in \mathcal{P}\left(\mathcal{B}_{3}(\alpha)\right)$, temos que

$b_{1}^{2}=\frac{\beta^{2}}{\lambda_{1}^{5}} a_{1}^{5}+\frac{\beta^{2}\left(\lambda_{1} \alpha-5 \lambda_{2}\right)}{\lambda_{1}^{7}} a^{6}$ e $a_{1}^{2} b_{1}=\frac{\beta}{\lambda_{1}^{3}} a^{5}-\frac{\beta\left(8 \lambda_{2}+\lambda\right)}{\lambda_{1}^{5}}$ com $\lambda_{1}, \beta \neq 0$.

Logo para $\alpha^{\prime}$ em $F, \mathcal{B}_{3}(\alpha)$ é isomorfa a $\mathcal{B}_{3}\left(\alpha^{\prime}\right)$, se e somente se $1=\frac{\beta^{2}}{\lambda_{1}^{5}}, \alpha^{\prime}=$ $\frac{\beta^{2}\left(\lambda_{1} \alpha-5 \lambda_{2}\right)}{\lambda_{1}^{7}}, 1=\frac{\beta}{\lambda_{1}^{3}}$ e $0=-\frac{\beta\left(8 \lambda_{2}+\lambda\right)}{\lambda_{1}^{5}}$. Portanto $\lambda=-8 \lambda_{2}, \beta=\lambda_{1}^{3}$ e $\alpha^{\prime}=$ $\alpha-\frac{5 \lambda_{2}}{\lambda_{1}}$.

Lema 4.38 Sejam $\alpha$ e $\alpha^{\prime}$ em F. Então

$$
\mathcal{B}_{2}(\alpha) \cong \mathcal{B}_{2}\left(\alpha^{\prime}\right)
$$

se, e somente se, existir $\rho$ não nulo tal que $\alpha^{\prime}=\rho^{2} \alpha$.

Prova: Seja $\alpha$ e $\alpha^{\prime}$ em $F$ e $(a, b)$ em $\mathcal{P}\left(\mathcal{B}_{2}(\alpha)\right)$ tal que $b^{2}=a^{4}+\alpha a^{6}$, $a^{2} b=a^{4}, a^{3} b=-a^{5}$ e $a^{4} b=a^{6}$. Então cada par $\left(a_{1}, b_{1}\right)$ em $\mathcal{P}(\mathcal{B})$, exprimese de maneira única na base determinada por $(a, b)$ como $a_{1}=\lambda b+\lambda_{1} a+$ $\lambda_{2} a^{2}+\lambda_{3} a^{3}+\lambda_{4} a^{4}+\lambda_{5} a^{5}+\lambda_{6} a^{6}$ e $b_{1}=\beta b+\beta_{1} a+\beta_{2} a^{2}+\beta_{3} a^{3}+\beta_{4} a^{4}+\beta_{5} a^{5}+\beta_{6} a^{6}$. As potências de $a_{1}$, dadas por (4.11), são 


$$
\begin{aligned}
a_{1}^{2}= & \lambda_{1}^{2} a^{2}+2 \lambda_{1} \lambda_{2} a^{3}+\left(\lambda^{2}+2 \lambda \lambda_{2}+2 \lambda_{1} \lambda_{3}+\lambda_{2}^{2}\right) a^{4}+\left(-2 \lambda \lambda_{3}+2 \lambda_{1} \lambda_{4}+\right. \\
& \left.2 \lambda_{2} \lambda_{3}\right) a^{5}+\left(\lambda^{2} \alpha+2 \lambda_{1} \lambda_{5}+2 \lambda_{2} \lambda_{4}+\lambda_{3}^{2}\right) a^{6}, \\
a_{1}^{3}= & \lambda_{1}^{3} a^{3}+\left(\lambda \lambda_{1}^{2}+3 \lambda_{1}^{2} \lambda_{2}\right) a^{4}+\left(\lambda_{1} \lambda^{2}+3 \lambda_{3} \lambda_{1}^{2}+3 \lambda_{1} \lambda_{2}^{2}\right) a^{5}+\left(\lambda^{3}+3 \lambda_{2} \lambda^{2}\right. \\
& \left.+3 \lambda \lambda_{2}^{2}+3 \lambda_{4} \lambda_{1}^{2}+6 \lambda_{1} \lambda_{2} \lambda_{3}+\lambda_{2}^{3}\right) a^{6}, \\
a_{1}^{4}= & \lambda_{1}^{4} a^{4}+4 \lambda_{1}^{3} \lambda_{2} a^{5}+\left(2 \lambda^{2} \lambda_{1}^{2}+4 \lambda \lambda_{1}^{2} \lambda_{2}+4 \lambda_{1}^{3} \lambda_{3}+6 \lambda_{2}^{2} \lambda_{1}^{2}\right) a^{6}, \\
a_{1}^{5}= & \lambda_{1}^{5} a^{5}+\left(\lambda \lambda_{1}^{4}+5 \lambda_{1}^{4} \lambda_{2}\right) a^{6}, \\
a_{1}^{6}= & \lambda_{1}^{6} a^{6} \neq 0,
\end{aligned}
$$

$\operatorname{logo} \lambda_{1} \neq 0$, pois $a_{1}$ tem nilíndice máximo.

$$
\begin{aligned}
a_{1} b_{1}= & \lambda \beta b^{2}+\lambda \beta_{2} a^{2} b+\lambda \beta_{3} a^{3} b+\lambda \beta_{4} a^{4} b+\lambda_{1} \beta_{1} a^{2}+\lambda_{1} \beta_{2} a^{3}+\lambda_{1} \beta_{3} a^{4}+ \\
& \lambda_{1} \beta_{4} a^{5}+\lambda_{1} \beta_{5} a^{6}+\lambda_{2} \beta a^{2} b+\lambda_{2} \beta_{1} a^{3}+\lambda_{2} \beta_{2} a^{4}+\lambda_{2} \beta_{3} a^{5}+\lambda_{2} \beta_{4} a^{6}+ \\
& \lambda_{3} \beta a^{3} b+\lambda_{3} \beta_{1} a^{4}+\lambda_{3} \beta_{2} a^{5}+\lambda_{3} \beta_{3} a^{6}+\lambda_{4} \beta a^{4} b+\lambda_{4} \beta_{1} a^{5}+\lambda_{4} \beta_{2} a^{6}+ \\
& \lambda_{5} \beta_{1} a^{6} \\
= & \lambda_{1} \beta_{1} a^{2}+\left(\lambda_{1} \beta_{2}+\lambda_{2} \beta_{1}\right) a^{3}+\left(\lambda \beta+\lambda \beta_{2}+\lambda_{2} \beta+\lambda_{1} \beta_{3}+\lambda_{2} \beta_{2}+\right. \\
& \left.\lambda_{3} \beta_{1}\right) a^{4}+\left(\lambda \beta_{3}-\lambda_{3} \beta+\lambda_{1} \beta_{4}+\lambda_{4} \beta_{1}+\lambda_{2} \beta_{3}+\lambda_{3} \beta_{2}\right) a^{5} \\
& +\left(\lambda \beta \alpha+\lambda \beta_{4}+\lambda_{4} \beta+\lambda_{1} \beta_{5}-\lambda_{5} \beta_{1}+\lambda_{2} \beta_{4}+\lambda_{3} \beta_{3}+\lambda_{4} \beta_{2}\right) a^{6} \\
= & 0
\end{aligned}
$$

$\log \mathrm{O} \beta_{1}=\beta_{2}=0, \beta_{3}=-\frac{\beta\left(\lambda+\lambda_{2}\right)}{\lambda_{1}}, \beta_{4}=-\frac{\beta\left(\lambda^{2}-\lambda_{1} \lambda_{3}-\lambda_{2}^{2}\right)}{\lambda_{1}^{2}} \mathrm{e}$

$$
\beta_{5}=\frac{\beta\left(\lambda \lambda_{1}^{2}-\lambda^{3}+\lambda \lambda_{2}^{2}+\lambda_{4} \lambda_{1}^{2}-\lambda_{2} \lambda^{2}+\lambda_{2}^{3}\right)}{\lambda_{1}^{3}} .
$$

Portanto

$$
\begin{aligned}
b_{1}= & \beta b-\frac{\beta\left(\lambda+\lambda_{2}\right)}{\lambda_{1}} a^{3}-\frac{\beta\left(\lambda^{2}-\lambda_{1} \lambda_{3}-\lambda_{2}^{2}\right)}{\lambda_{1}^{2}} a^{4} \\
& +\frac{\beta\left(\lambda \lambda_{1}^{2}-\lambda^{3}+\lambda \lambda_{2}^{2}+\lambda_{4} \lambda_{1}^{2}-\lambda_{2} \lambda^{2}+\lambda_{2}^{3}\right)}{\lambda_{1}^{3}} a^{5}+\beta_{6} a^{6},
\end{aligned}
$$


$\operatorname{com} \beta \neq 0 . \log 0$

$$
\begin{aligned}
b_{1}^{2} & =\beta^{2} b^{2}-2 \frac{\beta^{2}\left(\lambda+\lambda_{2}\right)}{\lambda_{1}} a^{3} b-\frac{2 \beta^{2}\left(\lambda^{2}-\lambda_{1} \lambda_{3}-\lambda_{2}^{2}\right)}{\lambda_{1}^{2}} a^{4} b+\frac{\beta^{2}\left(\lambda+\lambda_{2}\right)^{2}}{\lambda_{1}^{2}} a^{3} \\
& =\beta^{2} a^{4}+\frac{2 \beta^{2}\left(\lambda+\lambda_{2}\right)}{\lambda_{1}} a^{5}+\frac{\beta^{2}\left(\lambda_{1}^{2} \alpha-\lambda^{2}+2 \lambda_{1} \lambda_{3}+3 \lambda_{2}^{2}+2 \lambda \lambda_{2}\right)}{\lambda_{1}^{2}} a^{6},
\end{aligned}
$$

$\mathrm{e}$

$$
\begin{aligned}
a_{1}^{2} b_{1}= & \lambda_{1}^{2} \beta a^{b}+2 \lambda_{1} \lambda_{2} \beta a^{3} b+\left(\lambda^{2}+2 \lambda \lambda_{2}+2 \lambda_{1} \lambda_{3}+\lambda_{2}^{2}\right) a^{4} b+\frac{\lambda_{1}^{2} \beta\left(\lambda+\lambda_{2}\right)}{\lambda_{1}} a^{5} \\
& -\frac{\lambda_{1}^{2} \beta\left(\lambda^{2}-\lambda_{1} \lambda_{3}-\lambda_{2}^{2}\right)}{\lambda_{1}^{2}} a^{6}-\frac{2 \lambda_{1} \lambda_{2} \beta\left(\lambda+\lambda_{2}\right)}{\lambda_{1}} a^{6} \\
= & \beta \lambda_{1}^{2} a_{1}^{4}-\beta \lambda_{1}\left(3 \lambda_{2}+\lambda\right) a^{5}+3 \beta \lambda_{1} \lambda_{3} a^{6} .
\end{aligned}
$$

Seja $M$ a matriz cujas colunas são as coordenadas de $a_{1}^{2}, \ldots, a_{1}^{6}, b_{1}^{2}, a_{1}^{2} b_{1}$, em relação a $a^{2}, \ldots, a^{6}$. Então uma redução por linhas nos leva a matriz

$$
\left[\begin{array}{ccccccc}
1 & 0 & 0 & 0 & 0 & 0 & 0 \\
0 & 1 & 0 & 0 & 0 & 0 & 0 \\
0 & 0 & 1 & 0 & 0 & \frac{\beta^{2}}{\lambda_{1}^{3}} & \frac{\beta}{\lambda_{1}^{2}} \\
0 & 0 & 0 & 1 & 0 & -\frac{2 \beta^{2}\left(\lambda_{2}-\lambda\right)}{\lambda_{1}^{6}} & -\frac{\beta\left(7 \lambda_{2}+\lambda\right)}{\lambda_{1}^{4}} \\
0 & 0 & 0 & 0 & 1 & \frac{\beta^{2}\left(\alpha \lambda_{1}^{2}-5 \lambda^{2}-2 \lambda_{1} \lambda_{3}+7 \lambda_{2}^{2}-10 \lambda \lambda_{2}\right)}{\lambda_{1}^{4}} & -\frac{\beta\left(\lambda_{1} \lambda_{3}+\lambda^{2}-8 \lambda \lambda_{2}-29 \lambda_{2}^{2}\right)}{\lambda_{1}^{6}}
\end{array}\right] .
$$

Portanto para um $\alpha^{\prime}$ em $F, \mathcal{B}_{3}(\alpha) \cong \mathcal{B}_{3}\left(\alpha^{\prime}\right)$, se e somente se

$$
\begin{gathered}
1=\frac{\beta^{2} \alpha}{\lambda_{1}^{4}}, 0=-\frac{2 \beta^{2}\left(\lambda_{2}-\lambda\right)}{\lambda_{1}^{6}}, \alpha^{\prime}=\frac{\beta^{2}\left(\alpha \lambda_{1}^{2}-5 \lambda^{2}-2 \lambda_{1} \lambda_{3}+7 \lambda_{2}^{2}-10 \lambda \lambda_{2}\right)}{\lambda_{1}^{4}}, \\
1=\frac{\beta}{\lambda_{1}^{2}}, 0=-\frac{\beta\left(7 \lambda_{2}+\lambda\right)}{\lambda_{1}^{4}} \text { e } 0=-\frac{\beta\left(\lambda_{1} \lambda_{3}+\lambda^{2}-8 \lambda \lambda_{2}-29 \lambda_{2}^{2}\right)}{\lambda_{1}^{6}} .
\end{gathered}
$$

$\operatorname{Logo} \beta=\lambda_{1}^{2}, \quad \lambda_{2}=-\frac{1}{7} \lambda, \quad \lambda_{3}=\frac{76 \lambda^{2}}{49 \lambda_{1}}$ e $\lambda=\frac{7}{16} \lambda_{1}^{2}$ e assim obtemos que $\alpha^{\prime}=\frac{1}{\lambda_{1}^{2}} \alpha$, e dado que $\lambda_{1} \neq 0$ segue-se a demonstração do lema.

Temos finalmente o seguinte resultado para dimensão e nilíndice 7 . 
Teorema 4.39 Uma nilálgebra comutativa de p.a. de dimensão e nilíndice 7, é isomorfa a uma e somente uma álgebra com base $\left\{b, a, a^{2}, a^{3}, a^{4}, a^{5}, a^{6}\right\}$, e produtos não triviais e não zero conforme um dos casos que aparecem na seguinte lista:

$$
\begin{aligned}
& \mathcal{A}_{1}(\alpha): \quad b^{2}=a^{4}+\alpha a^{6}, \quad a^{2} b=a^{4}, \quad a^{3} b=-a^{5}, \quad a^{4} b=a^{6}, \quad\left[\rho^{2}\right] ; \\
& \mathcal{A}_{2}: \quad b^{2}=a^{5}, \quad a^{2} b=a^{5}, \quad a^{3} b=-a^{6} ; \\
& \mathcal{A}_{3}(\alpha): \quad b^{2}=\alpha a^{6}, \quad a^{2} b=a^{5}, \quad a^{3} b=-a^{6}, \quad[1] ; \\
& \mathcal{A}_{4}(\alpha): \quad b^{2}=a^{5}, \quad a^{2} b=\alpha a^{6}, \quad\left[\rho^{2}\right] ; \\
& \mathcal{A}_{5}(\alpha): \quad b^{2}=\alpha a^{6}, \quad \quad a^{2} b=a^{6}, \quad\left[\rho^{2}\right] ; \\
& \mathcal{A}_{6}(\alpha): \quad b^{2}=\alpha a^{6}, \quad\left[\rho^{2}\right] .
\end{aligned}
$$

\subsection{Classificação para dimensão oito.}

Nas seções anteriores mostramos explicitamente as constantes de estrutura de uma nilálgebra $\mathcal{B}$ em relação a base determinada por um par $(a, b)$ em $\mathcal{P}(\mathcal{B})$. Veremos a partir de esta seção que tais constantes tem um comportamento padrão. Embora seja possível, a partir da dimensão oito, dar um teorema geral caracterizando tais constantes, faremos este caso separado do caso geral, pois mesmo sendo a idéia a mesma que demonstra o caso geral, teremos de considerar algumas particularidades para dimensão 8.

O método utilizado para achar as classes de isomorfismos destas álgebras, será o mesmo utilizado para dimensão 5,6 e 7, sendo em este caso, algumas demonstrações muito mais simples. Uma vez que o método já foi muito trabalhado, daremos apenas as idéias principais.

Iniciaremos com o teorema que caracteriza tais constantes.

Teorema 4.40 Seja $\mathcal{B}$ uma nilálgebra comutativa de p.a. de dimensão e nilíndice 8 e $(a, b)$ em $\mathcal{P}(\mathcal{B})$. Então para a base determinada pelo par anterior, isto é, formada por $b$ e as potências de a, os produtos não triviais são 
dados por:

$$
\begin{aligned}
b^{2} & =\alpha_{6} a^{6}+\alpha_{7} a^{7}, \\
a^{2} b & =\gamma_{5} a^{5}+\gamma_{6} a^{6}+\gamma_{7} a^{7}, \\
a^{3} b & =-\gamma_{5} a^{6}-\gamma_{6} a^{7}, \\
a^{4} b & =\gamma_{5} a^{7} .
\end{aligned}
$$

Reciprocamente, se $\mathcal{B}$ é uma nilálgebra comutativa, com base $\left\{b, a, a^{2}, \ldots, a^{7}\right\}$, e produtos $a b=a^{k}=0, a^{i} a^{j}=a^{i+j}$, para todo $k \geq 8$ e $i, j \geq 1$, e (4.12), então $\mathcal{B}$ é de p.a. e nilíndice 8.

Prova: Seja $\mathcal{B}$ uma nilálgebra comutativa de p.a. de dimensão e nilíndice 8, e $(a, b)$ em $\mathcal{P}(\mathcal{B})$. Então $\overline{\mathcal{B}}=\mathcal{B} / A_{a}^{7}=\left\langle\bar{b}, \bar{a}, \bar{a}^{2}, \bar{a}^{3}, \bar{a}^{4}, \bar{a}^{5}, \bar{a}^{6}\right\rangle$. Pelo Teorema 4.27, temos que $\bar{b}^{2}=\gamma_{4}^{2} \bar{a}^{4}+\alpha_{5} \bar{a}^{5}+\alpha_{6} \bar{a}^{6}$ e $\bar{a}^{2} \bar{b}=\gamma_{4} \bar{a}^{4}+\gamma_{5} \bar{a}^{5}+\gamma_{6} \bar{a}^{6}$, logo

$$
b^{2}=\gamma_{4}^{2} a^{4}+\alpha_{5} a^{5}+\alpha_{6} a^{6}+\alpha_{7} a^{7} \quad \text { e } \quad a^{2} b=\gamma_{4} a^{4}+\gamma_{5} a^{5}+\gamma_{6} a^{6}+\gamma_{7} a^{7} .
$$

Por (4.1) a (4.5) temos que

$$
\begin{aligned}
a^{3} b & =-\gamma_{4} a^{5}-\gamma_{5} a^{6}-\gamma_{6} a^{7}, \\
a^{4} b & =\gamma_{4} a^{6}+\gamma_{5} a^{7}, \\
a^{5} b & =-\frac{5}{3} \gamma_{4} a^{7} \\
a^{6} b & =0
\end{aligned}
$$

$$
a^{2} b^{2}=\gamma_{4}^{2} a^{6}+\alpha_{5} a^{7}=\gamma_{4}^{2} a^{6}+\frac{1}{2}\left(\alpha_{5}-\frac{2}{5} \gamma_{4} \gamma_{5}\right) a^{7},
$$

de onde $\alpha_{5}=-\frac{2}{3} \gamma_{4} \gamma_{5}$, assim

$$
b^{2}=\gamma_{4}^{2} a^{4}-\frac{2}{3} \gamma_{4} \gamma_{5} a^{5}+\alpha_{6} a^{6}+\alpha_{7} a^{7} .
$$

Seja

$$
x=b+a+a^{2} .
$$




\section{Então}

$$
\begin{aligned}
x^{2}= & a^{2}+2 a^{3}+\left(\gamma_{4}^{2}+2 \gamma_{4}+1\right) a^{4}+\left(-\frac{2}{3} \gamma_{4} \gamma_{5}+2 \gamma_{5}\right) a^{5}+\left(2 \gamma_{6}+\alpha_{6}\right) a^{6} \\
& +\left(2 \gamma_{7}+\alpha_{7}\right) a^{7} \\
x^{3}= & a^{3}+\left(3+\gamma_{4}\right) a^{4}+\left(3+\gamma_{4}^{2}+\gamma_{5}\right) a^{5}+\left(1+3 \gamma_{4}-\frac{2}{3} \gamma_{4} \gamma_{5}+3 \gamma_{4}^{2}+\gamma_{4}^{3}+\gamma_{6}\right) a^{6} \\
& +\left(-2 \gamma_{4} \gamma_{5}+\frac{19}{9} \gamma_{4} \gamma_{5}^{2}+3 \gamma_{5}+\gamma_{7}+\alpha_{6}\right) a^{7}, \\
x^{4}= & a^{4}+4 a^{5}+\left(6+4 \gamma_{4}+2 \gamma_{4}^{2}\right) a^{6}+\left(4-2 \gamma_{4}-\frac{4}{3} \gamma_{4} \gamma_{5}+4 \gamma_{4}^{2}-\frac{2}{3} \gamma_{4}^{3}+4 \gamma_{5}\right) a^{7},
\end{aligned}
$$

$\mathrm{e}$

$$
x^{2} x^{2}=a^{4}+4 a^{5}+\left(6+4 \gamma_{4}+2 \gamma_{4}^{2}\right) a^{6}+\left(4+8 \gamma_{4}-\frac{4}{3} \gamma_{4} \gamma_{5}+4 \gamma_{4}^{2}+4 \gamma_{5}\right) a^{7},
$$

e lembrando que $\mathcal{B}$ é de p.a. temos que

$$
0=x^{4}-x^{2} x^{2}=\left(-\frac{2}{3} \gamma_{4}^{2}-10\right) \gamma_{4} a^{7}
$$

do que segue que $\gamma_{4}=0$, portanto $b^{2}=\alpha_{6} a^{6}+\alpha_{7} a^{7}, a^{2} b=\gamma_{5} a^{5}+\gamma_{6} a^{6}+\gamma_{7} a^{7}$, $a^{3} b=-\gamma_{5} a^{6}-\gamma_{6} a^{7}, a^{4} b=\gamma_{5} a^{7}$ e $a^{t} b=0$, para $t \geq 5$.

Suponhamos agora que $\mathcal{B}$ é uma nilálgebra comutativa com base $\left\{b, a, a^{2}, a^{3}\right.$, $\left.a^{4}, a^{5}, a^{6}, a^{7}\right\}$ e produtos $a b=a^{k}=0, a^{i} a^{j}=a^{i+j}$, para todo $k \geq 8$ e to$\operatorname{dos}$ os $i, j \geq 1$, e (4.12). Se $x=\lambda b+\sum_{i=1}^{7} \lambda_{i} a^{i}$, então

$$
\begin{aligned}
x^{2}= & \lambda_{1}^{2} a^{2}+2 \lambda_{1} \lambda_{2} a^{3}+\left(2 \lambda_{1} \lambda_{3}+\lambda_{2}^{2}\right) a^{4}+\left(2 \lambda \lambda_{2} \gamma_{5}+2 \lambda_{1} \lambda_{4}+2 \lambda_{2} \lambda_{3}\right) a^{5} \\
& +\left(\lambda^{2} \alpha_{6}+2 \lambda \lambda_{2} \gamma_{6}-2 \lambda \lambda_{3} \gamma_{5}+2 \lambda_{1} \lambda_{5}+2 \lambda_{2} \lambda_{4}+\lambda_{3}^{2}\right) a^{6}+ \\
& \left(\lambda^{2} \alpha_{7}+2 \lambda \lambda_{2} \gamma_{7}-2 \lambda \lambda_{3} \gamma_{6}+2 \lambda \lambda_{4} \gamma_{5}+2 \lambda_{1} \lambda_{6}+2 \lambda_{2} \lambda_{5}+2 \lambda_{3} \lambda_{4}\right) a^{7}, \\
x^{3}= & \lambda_{1}^{3} a^{3}+3 \lambda_{1}^{2} \lambda_{2} a^{4}+\left(\lambda \lambda_{1}^{2} \gamma_{5}+3 \lambda_{3} \lambda_{1}^{2}+3 \lambda_{1} \lambda_{2}^{2}\right) a^{5}+\left(\lambda \lambda_{1}^{2} \gamma_{6}+3 \lambda_{1}^{2} \lambda_{4}+\right. \\
& \left.6 \lambda_{1} \lambda_{2} \lambda_{3}+\lambda_{2}^{3}\right) a^{6}+\left(\lambda \lambda_{1}^{2} \gamma_{7}+3 \lambda \lambda_{2}^{2} \gamma_{5}+\lambda_{1} \lambda^{2} \alpha_{6}+3 \lambda_{1}^{2} \lambda_{5}+6 \lambda_{1} \lambda_{2} \lambda_{4}\right. \\
& \left.+3 \lambda_{1} \lambda_{3}^{2}+3 \lambda_{2}^{2} \lambda_{3}\right) a^{7}, \\
x^{4}= & \lambda_{1}^{4} a^{4}+4 \lambda_{1}^{3} \lambda_{2} a^{5}+\left(4 \lambda_{1}^{3} \lambda_{3}+6 \lambda_{1}^{2} \lambda_{2}^{2}\right) a^{6}+\left(4 \lambda \lambda_{1}^{2} \lambda_{2} \gamma_{5}+4 \lambda_{1}^{3} \lambda_{4}\right. \\
& \left.+12 \lambda_{1}^{2} \lambda_{2} \lambda_{3}+4 \lambda_{1} \lambda_{2}^{3}\right) a^{7},
\end{aligned}
$$




$$
\begin{aligned}
x^{5}= & \lambda_{1}^{5} a^{5}+5 \lambda_{1}^{4} \lambda_{2} a^{6}+\left(\lambda \lambda_{1}^{4} \gamma_{5}+5 \lambda_{1}^{4} \lambda_{3}+10 \lambda_{1}^{3} \lambda_{2}^{2}\right) a^{7}, \\
x^{6}= & \lambda_{1}^{6} a^{6}+6 \lambda_{1}^{5} \lambda_{2} a^{7}, \\
x^{7}= & \lambda_{1}^{7} a^{7} \\
x^{8}= & 0, \\
x^{2} x^{2}= & \lambda_{1}^{4} a^{4}+4 \lambda_{1}^{3} \lambda_{2} a^{5}++\left(4 \lambda_{1}^{3} \lambda_{3}+6 \lambda_{1}^{2} \lambda_{2}^{2}\right) a^{6}+\left(4 \lambda \lambda_{1}^{2} \lambda_{2} \gamma_{5}+4 \lambda_{1}^{3} \lambda_{4}\right. \\
& \left.+12 \lambda_{1}^{2} \lambda_{2} \lambda_{3}+4 \lambda_{1} \lambda_{2}^{3}\right) a^{7},
\end{aligned}
$$

assim $x^{4}=x^{2} x^{2}$ e podemos concluir que $\mathcal{B}$ é de p.a. e tem nilíndice 8.

Denotaremos por $\mathcal{B}\left(\alpha_{6}, \alpha_{7}, \gamma_{5}, \gamma_{6}, \gamma_{7}\right)$ a álgebra dada no lema anterior. Para esta álgebra temos que a matriz coordenada por linhas de $L_{b}$, em relação à base $\left\{b, a, a^{2}, a^{3}, a^{4}, a^{5}, a^{6}, a^{7}\right\}$ é

$$
\left(\begin{array}{c|c}
0 & \mathrm{M} \\
\hline 0 & 0
\end{array}\right) \quad \text { sendo } \quad M=\left(\begin{array}{ccc}
0 & \alpha_{6} & \alpha_{7} \\
0 & 0 & 0 \\
\gamma_{5} & \gamma_{6} & \gamma_{7} \\
0 & -\gamma_{5} & -\gamma_{6} \\
0 & 0 & \gamma_{5}
\end{array}\right)
$$

Passemos a estudar as classes de isomorfismo para as álgebras que tem a forma anterior. Sejam $(a, b)$ em $\mathcal{P}(\mathcal{B})$. Então se definimos para $\lambda$ e $\beta$ em $F^{*}$, os elementos $a_{1}:=\lambda a$ e $b_{1}:=\beta b$, obtemos que

$$
\mathcal{B}\left(\alpha_{6}, \alpha_{7}, \gamma_{5}, \gamma_{6}, \gamma_{7}\right) \cong \mathcal{B}\left(\frac{\beta^{2}}{\lambda^{6}} \alpha_{6}, \frac{\beta^{2}}{\lambda^{7}} \alpha_{7}, \frac{\beta}{\lambda^{3}} \gamma_{5}, \frac{\beta}{\lambda^{4}} \gamma_{6}, \frac{\beta}{\lambda^{5}} \gamma_{7}\right)
$$

Lema 4.41 Sejam $\rho_{1}$ e $\rho_{2}$ em F. Então $\mathcal{B}\left(\alpha_{6}, \alpha_{7}, \gamma_{5}, \gamma_{6}, \gamma_{7}\right)$ é isomorfa à álgebra

$$
\mathcal{B}\left(\alpha_{6}, \alpha_{7}-2 \rho_{1}\left(\gamma_{5}^{2}+3 \alpha_{6}\right), \gamma_{5}, \gamma_{6}-8 \rho_{1} \gamma_{5}, \gamma_{7}+\gamma_{5}\left(38 \rho_{1}^{2}-2 \rho_{2}\right)-9 \rho_{1} \gamma_{6}\right) .
$$

Prova: Seja $\mathcal{B}$ a álgebra $\mathcal{B}\left(\alpha_{5}, \alpha_{6}, \gamma_{4}, \gamma_{5}, \gamma_{6}\right)$ em relação ao par $(a, b)$ em $\mathcal{P}(\mathcal{B})$. Sejam $\rho_{1}$ e $\rho_{2}$ em $F$,

$$
a_{1}:=a+\rho_{1} a^{2}+\rho_{2} a^{3}
$$


e

$$
b_{1}:=b-\rho_{1} \gamma_{5} a^{4}+\left(-\rho_{1} \gamma_{6}+\rho_{1}^{2} \gamma_{5}+\rho_{2} \gamma_{5}\right) a^{5}-\left(\rho_{1} \gamma_{7}-\rho_{2} \gamma_{6}-\rho_{1}^{2} \gamma_{6}+\rho_{1}^{3} \gamma_{5}\right) a^{6} .
$$

Um calculo direto nos mostra que $a_{1}^{7}=a^{7} \neq 0$ e $a_{1} b_{1}=0$, e uma vez que é claro que $b_{1} \notin A_{a_{1}}$, temos que $\left(a_{1}, b_{1}\right) \in \mathcal{P}(\mathcal{B})$. Calculando os produtos $b_{1}^{2}$ e $a_{1}^{2} b_{1}$, obtemos que

$$
b_{1}^{2}=\alpha_{6} a^{6}+\left(\alpha_{7}-2 \rho_{1} \gamma_{5}^{2}\right) a^{7}=\alpha_{6} a_{1}^{6}+\left[\alpha_{7}-2 \rho_{1}\left(\gamma_{5}^{2}+3 \alpha_{6}\right)\right] a_{1}^{7}
$$

e

$$
\begin{aligned}
a_{1}^{2} b_{1} & =\gamma_{5} a^{5}+\left(\gamma_{6}-3 \rho_{1} \gamma_{5}\right) a^{6}+\left(\gamma_{7}-3 \rho_{1} \gamma_{6}+3 \rho_{2} \gamma_{5}\right) a^{7} \\
& =\gamma_{5} a_{1}^{5}+\left(\gamma_{6}-8 \rho_{1} \gamma_{5}\right) a_{1}^{6}+\left[\gamma_{7}+\gamma_{5}\left(38 \rho_{1}^{2}-2 \rho_{2}\right)-9 \rho_{1} \gamma_{6}\right] a_{1}^{7} .
\end{aligned}
$$

Isto prova o lema.

Por (4.13) podemos supor, a menos de isomorfismos, que $\gamma_{5}=0$ ou $\gamma_{5}=1$. Acharemos as classes de isomorfismos considerando estes dois casos por separado.

- $\left(\gamma_{5}=0\right.$.) Neste caso temos pelo Lema 4.41 que $\mathcal{B}\left(\alpha_{6}, \alpha_{7}, 0, \gamma_{6}, \gamma_{7}\right) \cong$ $\mathcal{B}\left(\alpha_{6}, \alpha_{7}-6 \rho_{1} \alpha_{6}, 0, \gamma_{6}, \gamma_{7}-9 \rho_{1} \gamma_{6}\right)$.

- Se $\gamma_{6} \neq 0$, então considerando $\rho_{1}=\frac{\gamma_{7}}{9 \gamma_{6}}$, no Lema 4.41 e fazendo $\lambda=\gamma_{6}$ e $\beta=\gamma_{6}^{3}$ em (4.13), obtemos que $\mathcal{B}\left(\alpha_{6}, \alpha_{7}, 0, \gamma_{6}, \gamma_{7}\right)$ é isomorfa à álgebra $\mathcal{B}\left(\alpha_{6}, \alpha_{7}^{\prime}, 0,1,0\right)$. Se $\alpha_{7}^{\prime}$ é não nulo, então fazendo $\lambda=\frac{1}{\alpha_{7}^{\prime}}$ e $\beta=\frac{1}{\alpha_{7}^{\prime 4}}$, temos que

$$
\mathcal{B}\left(\alpha_{6}, \alpha_{7}^{\prime}, 0,1,0\right) \cong \mathcal{B}\left(\alpha_{6}^{\prime}, 1,0,1,0\right),
$$

e se $\alpha_{7}^{\prime}=0$, então temos a álgebra $\mathcal{B}\left(\alpha_{6}, 0,0,1,0\right)$.

- Se $\gamma_{6}=0$, temos a álgebra $\mathcal{B}\left(\alpha_{6}, \alpha_{7}-6 \rho_{1} \alpha_{6}, 0,0, \gamma_{7}\right)$. Se $\alpha_{6}$ é não nulo e $\gamma_{7} \neq 0$, então considerando $\rho_{1}=\frac{\alpha_{7}}{6 \alpha_{6}}, \lambda=\gamma_{7}$ e $\beta=\gamma_{7}^{4}$, obtemos que

$$
\mathcal{B}\left(\alpha_{6}, \alpha_{7}, 0, \gamma_{6}, \gamma_{7}\right) \cong \mathcal{B}\left(\alpha_{6}^{\prime}, 0,0,0,1\right) \quad \operatorname{com} \quad \alpha_{6}^{\prime} \neq 0,
$$


e se $\gamma_{7}=0$, temos a álgebra $\mathcal{B}\left(\alpha_{6}, 0,0,0,0\right)$ com $\alpha_{6} \neq 0$.

Se $\alpha_{6}=0$, temos a álgebra $\mathcal{B}\left(0, \alpha_{7}, 0,0, \gamma_{7}\right)$. Se $\gamma_{7} \neq 0$, então considerando $\lambda=\gamma_{7}$ e $\beta=\gamma_{7}^{4}$, obtemos a álgebra $\mathcal{B}\left(0, \alpha_{7}^{\prime}, 0,0,1\right)$. Se $\gamma_{7}=0$, e $\alpha_{7} \neq 0$, então fazendo $\lambda=\alpha_{7}$ e $\beta=\alpha_{7}^{3}$, obtemos o isomorfismo com a álgebra $\mathcal{B}(0,1,0,0,0)$.

- $\left(\gamma_{5} \neq 0\right.$.) Então fazendo $\rho_{1}=\frac{\gamma_{6}}{8 \gamma_{5}}, \rho_{2}=\frac{32 \gamma_{5} \gamma_{7}-17 \gamma_{6}^{2}}{64 \gamma_{5}^{2}}, \lambda=\gamma_{5}$ e $\beta=\gamma_{5}^{2}$, obtemos o isomorfismo com a álgebra $\mathcal{B}\left(\alpha_{6}^{\prime}, \alpha_{7}^{\prime}, 1,0,0\right)$. Se $\alpha_{7}^{\prime} \neq 0$, então considerando $\lambda=\alpha_{7}^{\prime}$ e $\beta=\alpha_{7}^{\prime 3}$, obtemos o isomorfismo com a álgebra $\mathcal{B}\left(\alpha_{6}^{\prime}, 1,1,0,0\right)$. Se $\alpha_{7}^{\prime}=0$, temos a álgebra $\mathcal{B}\left(\alpha_{6}^{\prime}, 0,1,0,0\right)$.

Assim se $\mathcal{B}$ é uma nilálgebra comutativa de p.a. de dimensão e nilíndice 8, então existe uma base da forma $\left\{b, a, a^{2}, \ldots, a^{7}\right\}$, com produtos não triviais dados por um da seguinte lista:

$$
\begin{aligned}
& \mathcal{B}_{1}(\alpha): \quad b^{2}=\alpha a^{6}+a^{7}, \quad a^{2} b=a^{5}, \quad a^{3} b=-a^{6}, \quad a^{4} b=a^{7} ; \\
& \mathcal{B}_{2}(\alpha): \quad b^{2}=\alpha a^{6}, \quad a^{2} b=a^{5}, \quad a^{3} b=-a^{6}, \quad a^{4} b=a^{7} ; \\
& \mathcal{B}_{3}(\alpha): \quad b^{2}=\alpha a^{6}+a^{7}, \quad a^{2} b=a^{6}, \quad a^{3} b=-a^{7} ; \\
& \mathcal{B}_{4}(\alpha): \quad b^{2}=\alpha a^{6}, \quad a^{2} b=a^{6}, \quad a^{3} b=-a^{7} ; \\
& \mathcal{B}_{5}(\alpha): \quad b^{2}=\alpha a^{6}, \quad a^{2} b=a^{7}, \quad \alpha \neq 0 ; \\
& \mathcal{B}_{6}(\alpha): \quad b^{2}=\alpha a^{6}, \quad \alpha \neq 0 ; \\
& \mathcal{B}_{7}(\alpha): \quad b^{2}=\alpha a^{7}, \quad a^{2} b=a^{7} \\
& \mathcal{B}_{8}: \quad b^{2}=a^{7} \\
& \mathcal{B}_{9}:
\end{aligned}
$$

e os outros produtos não triviais são zero. Assim temos que toda álgebra desta classe é isomorfa a alguma da lista anterior. Estudemos as condições de isomorfismo das álgebras desta lista.

Claramente $\mathcal{B}_{9}$ não é isomorfa com as outras álgebras. Se $\overline{\mathcal{B}}_{i}=\mathcal{B} / \operatorname{Anul}\left(\mathcal{B}_{i}\right)$, então temos que a dimensão do anulador de $\overline{\mathcal{B}}_{7}$ e $\overline{\mathcal{B}}_{8}$ é 2 e das outras $\overline{\mathcal{B}}_{i}$ é 
1. Isto nos separa $\mathcal{B}_{7}$ e $\mathcal{B}_{8}$ das restantes álgebras. Observamos que $\overline{\mathcal{B}}_{1}$ e $\overline{\mathcal{B}}_{2}$ correspondem à álgebra $\mathcal{A}_{3}$, as álgebras $\overline{\mathcal{B}}_{3}$ e $\overline{\mathcal{B}}_{4}$ correspondem à álgebra $\mathcal{A}_{5} \mathrm{e}$ $\overline{\mathcal{B}}_{5}, \overline{\mathcal{B}}_{6}$ correspondem à álgebra $\mathcal{A}_{6}$, do Teorema 4.39 , que não são isomorfas. Assim temos as álgebras da lista anterior dividida em quatro conjuntos de álgebras não isomorfas, eles são:

$$
C 1: \mathcal{B}_{1}, \mathcal{B}_{2} . \quad C 2: \mathcal{B}_{3}, \mathcal{B}_{4} . \quad C 3: \mathcal{B}_{5}, \mathcal{B}_{6} . \quad C 4: \mathcal{B}_{7}, \mathcal{B}_{8} .
$$

Os resultados que daremos a continuação serão apresentados sem suas provas, que seguem os mesmos alinhamentos dos lemas das seções anteriores. Portanto no caso de mostrar o isomorfismo entre duas álgebras, daremos especificamente os elementos $a_{1}$ e $b_{1}$ que nos levam ao isomorfismo, sem fazer as contas que verificam ele. Igualmente quando precisemos calcular as constantes de estrutura das álgebras $\mathcal{B}_{i}$, em relação a base determinada pelo par $\left(a_{1}, b_{1}\right)$, indicaremos os produtos $b_{1}^{2}$ e $a_{1}^{2} b_{1}$ diretamente. Passemos agora a estudar cada um dos conjuntos anteriores.

Para o conjunto $C 3$, temos que as álgebras deste conjunto são isomorfas. Basta fixar a base determinada pelo par $(a, b)$ em $\mathcal{P}\left(\mathcal{B}_{6}\right)$ e definir em termos desta base os seguintes elementos

$$
a_{1}:=-\frac{1}{\alpha} b+a \text { e } b_{1}:=b+a^{5}
$$

para termos o isomorfismo. Assim para $\alpha$ não nulo

$$
\mathcal{B}_{5}(\alpha) \cong \mathcal{B}_{6}(\alpha)
$$

Analisando as álgebras do conjunto $C 2$, temos que para $\alpha$ não nulo, as álgebras $\mathcal{B}_{3}(\alpha)$ e $\mathcal{B}_{4}(\alpha)$ são isomorfas. De fato seja $\alpha \neq 0$ e $(a, b)$ um elemento de $\mathcal{P}\left(\mathcal{B}_{4}(\alpha)\right)$. Então se definimos, em termos da base determinada pelo par $(a, b)$, os elementos

$$
a_{1}:=\frac{3}{2 \alpha^{2}} b+a-\frac{1}{6 \alpha} a^{2} \text { e } b_{1}:=b-\frac{4}{3 \alpha} a^{5}-\frac{2}{9 \alpha^{2}} a^{6},
$$

temos que $\left(a_{1}, b_{1}\right) \in \mathcal{P}\left(\mathcal{B}_{4}(\alpha)\right), \quad b_{1}^{2}=\alpha a_{1}^{6}+a_{1}^{7}$ e $a_{1}^{2} b_{1}=a_{1}^{7}$, o que nos da o isomorfismo. Isto é,

$$
\mathcal{B}_{3}(\alpha) \cong \mathcal{B}_{4}(\alpha) \text { para } \alpha \in F^{*}
$$


Resta então analisar o caso em que $\alpha$ é nulo. O seguinte lema nos mostra que para $\alpha=0$, as álgebras, que denotaremos simplesmente por $\mathcal{B}_{3}$ e $\mathcal{B}_{4}$, não são isomorfas.

Lema 4.42 Para cada par $\left(a_{1}, b_{1}\right)$ em $\mathcal{P}\left(\mathcal{B}_{4}(\alpha)\right)$

$b_{1}^{2}=\frac{\beta^{2}}{\lambda_{1}^{6}} \alpha a_{1}^{6}-\frac{6 \beta^{2} \lambda_{2}}{\lambda_{1}^{8}} \alpha a_{1}^{7} \quad$ e $a_{1}^{2} b_{1}=\frac{\beta}{\lambda_{1}^{4}} a_{1}^{6}-\frac{\beta\left(9 \lambda_{2}+\lambda \alpha\right)}{\lambda_{1}^{6}} a_{1}^{7} \quad$ com $\lambda_{1}, \beta \in F^{*}$.

Do lema anterior temos que se $\alpha=0$, então $b_{1}^{2}=0$ o que nos mostra que

$$
\mathcal{B}_{4} \nsucceq \mathcal{B}_{3} \text {. }
$$

Se $\alpha$ é diferente de zero, temos que para um $\alpha^{\prime}$ em $F^{*}, \mathcal{B}_{4}(\alpha)$ é isomorfa a $\mathcal{B}_{4}\left(\alpha^{\prime}\right)$, se e somente se, $\lambda_{2}=\lambda=0, \beta=\lambda_{1}^{4}$, e consequentemente $\alpha^{\prime}=\lambda_{1}^{2} \alpha$.

Corolário 4.43 Sejam $\alpha$ e $\alpha^{\prime}$ não nulos. Então

$$
\mathcal{B}_{4}(\alpha) \cong \mathcal{B}_{4}\left(\alpha^{\prime}\right)
$$

se e somente se, existir $\rho$ não nulo tal que $\alpha^{\prime}=\rho^{2} \alpha$.

Analisando agora as álgebras do conjunto $C 4$, vemos que elas não são isomorfas. Isto segue-se do fato de, para cada par $\left(a_{1}, b_{1}\right)$ em $\mathcal{P}\left(\mathcal{B}_{8}\right)$, termos que

$$
b_{1}^{2}=\frac{\beta^{2}}{\lambda_{1}^{7}} a_{1}^{7} \quad \text { e } a_{1}^{2} b_{1}=0, \quad \text { com } \quad \lambda_{1}, \beta \in F^{*} .
$$

Portanto, é claro que

$$
\mathcal{B}_{8} \nsucceq \mathcal{B}_{7} \text {. }
$$

Resta então analisar as álgebras do conjunto $C 1$. O seguinte lema mostrará que as duas álgebras deste conjunto não são isomorfas.

Lema 4.44 Para cada par $\left(a_{1}, b_{1}\right)$ em $\mathcal{P}\left(\mathcal{B}_{2}(\alpha)\right)$,

$$
b_{1}^{2}=\frac{\beta^{2}}{\lambda_{1}^{6}} \alpha a_{1}^{6}-\frac{2 \beta^{2} \lambda_{2}(1+3 \alpha)}{\lambda_{1}^{8}} a_{1}^{7}
$$

$e$

$$
a_{1}^{2} b_{1}=\frac{\beta}{\lambda_{1}^{3}} a_{1}^{5}-\frac{8 \beta \lambda_{2}}{\lambda_{1}^{5}} a_{1}^{6}-\frac{\beta\left(2 \lambda_{1} \lambda_{3}+\lambda \lambda_{1} \alpha+\lambda \lambda_{1}-38 \lambda_{2}^{2}\right)}{\lambda_{1}^{7}} a_{1}^{7} \quad \text { com } \quad \lambda_{1}, \beta \in F^{*} .
$$


Assim, se $\mathcal{B}_{2}$ fosse isomorfa com $\mathcal{B}_{1}$, então $-\frac{8 \beta \lambda_{2}}{\lambda_{1}^{5}}=0$ e $-\frac{2 \beta^{2} \lambda_{2}(1+3 \alpha)}{\lambda_{1}^{8}}=1$, isto é, $\lambda_{2}=0$, e assim $1=\frac{\beta^{2}}{\lambda_{1}^{6}} \alpha=0$. Portanto

$$
\mathcal{B}_{2} \nsucceq \mathcal{B}_{1}
$$

Por outro lado temos que para um $\alpha^{\prime}$ em $F, \mathcal{B}_{2}(\alpha)$ é isomorfa com $\mathcal{B}_{2}\left(\alpha^{\prime}\right)$ se, e somente se, $\lambda_{2}=0, \lambda_{3}=-\frac{\lambda(\alpha+1)}{2}$ e $\beta=\lambda_{1}^{3}$, portanto $\alpha^{\prime}=\alpha$. Assim para todo $\alpha$ e $\alpha^{\prime}$ em $F$,

$$
\mathcal{B}_{2}(\alpha) \cong \mathcal{B}_{2}\left(\alpha^{\prime}\right) \text { se, e somente se, } \alpha=\alpha^{\prime}
$$

Com isto terminamos o análise das classes de isomorfismo. Passemos agora a estudar as classes dadas pelas álgebras $\mathcal{B}_{1}, \mathcal{B}_{6}$ e $\mathcal{B}_{7}$.

Seja $\alpha$ em $F$. Para cada par $\left(a_{1}, b_{1}\right)$ em $\mathcal{P}\left(\mathcal{B}_{1}(\alpha)\right)$

$$
b_{1}^{2}=\frac{\beta^{2}}{\lambda_{1}^{6}} \alpha a_{1}^{6}+\frac{\beta^{2}\left(\lambda_{1}-2 \lambda_{2}-6 \lambda_{2} \alpha\right)}{\lambda_{1}^{8}} a_{1}^{7}
$$

$\mathrm{e}$

$a_{1}^{2} b_{1}=\frac{\beta}{\lambda_{1}^{3}} a_{1}^{5}-\frac{8 \beta \lambda_{2}}{\lambda_{1}^{5}} a_{1}^{6}-\frac{\beta\left(2 \lambda_{1} \lambda_{3}+\lambda \lambda_{1} \alpha+\lambda \lambda_{1}-38 \lambda_{2}^{2}\right)}{\lambda_{1}^{7}} a_{1}^{7}$ com $\lambda_{1}, \beta \in F^{*}$.

Portanto temos que para um $\alpha^{\prime}$ em $F$, a álgebra $\mathcal{B}_{1}(\alpha)$ será isomorfa com $\mathcal{B}_{1}\left(\alpha^{\prime}\right)$ se e somente se, $\lambda_{2}=0, \lambda_{3}=-\frac{\lambda(\alpha+1)}{2}$ e $\beta=\lambda_{1}^{3}$, consequentemente $\alpha=\alpha^{\prime}$. Portanto temos que para $\alpha$ e $\alpha^{\prime}$ em $F$

$$
\mathcal{B}_{1}(\alpha) \cong \mathcal{B}_{1}\left(\alpha^{\prime}\right) \text { se, e somente se, } \alpha=\alpha^{\prime} .
$$

Seja $\alpha$ não nulo. Para cada par $\left(a_{1}, b_{1}\right)$ em $\mathcal{P}\left(\mathcal{B}_{6}(\alpha)\right)$

$$
b_{1}^{2}=\frac{\beta^{2}}{\lambda_{1}^{6}} \alpha a_{1}^{6}-\frac{6 \beta^{2} \lambda_{2} \alpha}{\lambda_{1}^{8}} a_{1}^{7} \text { e } a_{1}^{2} b_{1}=-\frac{\lambda \beta}{\lambda_{1}^{6}} \alpha a_{1}^{7} \quad \text { com } \quad \lambda_{1}, \beta \in F^{*} .
$$

Neste caso é claro que para um $\alpha^{\prime}$ em $F^{*}$,

$$
\mathcal{B}_{6}(\alpha) \cong \mathcal{B}_{6}\left(\alpha^{\prime}\right)
$$

se, e somente se, existir $\rho \in F^{*}$ tal que $\alpha=\rho^{2} \alpha^{\prime}$. 
Finalmente para um $\alpha$ qualquer em $F$, temos para $\mathcal{B}_{7}(\alpha)$ que para cada $\operatorname{par}\left(a_{1}, b_{1}\right)$ em $\mathcal{P}\left(\mathcal{B}_{7}(\alpha)\right)$

$$
b_{1}^{2}=\frac{\beta^{2}}{\lambda_{1}^{7}} \alpha a_{1}^{7} \quad \text { e } a_{1}^{2} b_{1}=\frac{\beta}{\lambda_{1}^{5}} a_{1}^{7} \quad \text { com } \quad \lambda_{1}, \beta \in F^{*} .
$$

Logo para um $\alpha^{\prime}$ em $F$, as álgebras $\mathcal{B}_{7}(\alpha)$ e $\mathcal{B}_{7}\left(\alpha^{\prime}\right)$ serão isomorfas se, e somente se, $\beta=\lambda_{1}^{5}$ e $\alpha^{\prime}=\frac{\beta^{2}}{\lambda_{1}^{7}} \alpha=\frac{\lambda_{1}^{10}}{\lambda_{1}^{7}} \alpha=\lambda_{1}^{3} \alpha$. Portanto para todo $\alpha$ e $\alpha^{\prime}$ em $F$

$$
\mathcal{B}_{7}(\alpha) \cong \mathcal{B}_{7}\left(\alpha^{\prime}\right)
$$

se, e somente se, existir $\rho \in F^{*}$ tal que $\alpha^{\prime}=\rho^{3} \alpha$.

De todo o anterior temos o seguinte resultado para dimensão e nilíndice 8.

Teorema 4.45 Uma nilálgebra comutativa de p.a. de dimensão e nilíndice 8, é isomorfa a uma e somente uma álgebra com base $\left\{b, a, a^{2}, a^{3}, a^{4}, a^{5}, a^{6}, a^{7}\right\}$, e produtos não triviais e não zero conforme um dos casos que aparecem na seguinte lista:

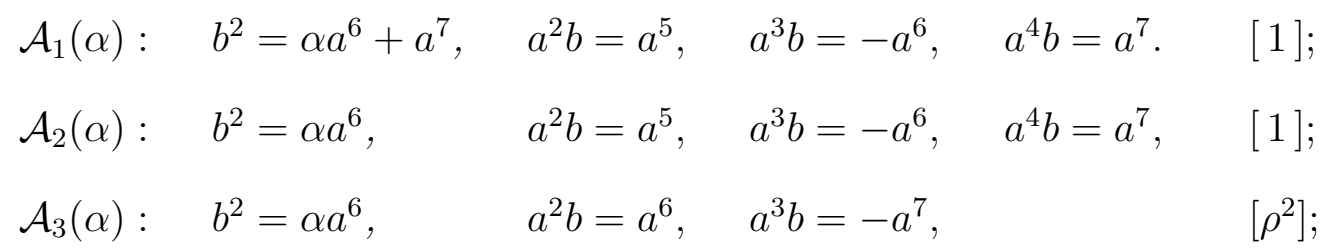

$\mathcal{A}_{4}: \quad a^{2} b=a^{6}, \quad a^{3} b=-a^{7} ;$

$\mathcal{A}_{5}(\alpha): \quad b^{2}=\alpha a^{6}$,

$\mathcal{A}_{6}: \quad b^{2}=a^{7}, \quad a^{2} b=a^{6}, \quad a^{3} b=-a^{7} ;$

$\mathcal{A}_{7}(\alpha): \quad b^{2}=\alpha a^{7}, \quad a^{2} b=a^{7}$

$\left[\rho^{3}\right]$

$\mathcal{A}_{8}: \quad b^{2}=a^{7}$ 


\subsection{Classificação para dimensão maior ou igual a nove.}

Finalmente, daremos nesta seção, o resultado geral que caracteriza as constantes de estrutura de uma nilálgebra $\mathcal{B}$ de dimensão e nilíndice $n$, na base determinada pelo par $(a, b)$ em $\mathcal{P}(\mathcal{B})$. Além disso daremos dois teoremas de classificação para estas álgebras, os quais foram separados para $n$ par e para $n$ ímpar. Para facilitar a demonstração do teorema geral, demonstraremos primeiro o teorema para dimensão 9 e utilizaremos ele na prova do caso geral.

Teorema 4.46 Seja $\mathcal{B}$ uma nilálgebra comutativa de p.a. de dimensão e nilíndice 9 e $(a, b)$ em $\mathcal{P}(\mathcal{B})$. Então para a base $\left\{b, a, a^{2}, \ldots, a^{8}\right\}$ de $\mathcal{B}$ os produtos não triviais são dados por:

$$
\begin{aligned}
b^{2} & =\alpha_{7} a^{7}+\alpha_{8} a^{8}, \\
a^{2} b & =\gamma_{6} a^{6}+\gamma_{7} a^{7}+\gamma_{8} a^{8}, \\
a^{3} b & =-\gamma_{6} a^{7}-\gamma_{7} a^{8}, \\
a^{4} b & =\gamma_{6} a^{8} .
\end{aligned}
$$

Reciprocamente, se $\mathcal{B}$ é uma nilálgebra comutativa, com base $\left\{b, a, a^{2}, \ldots, a^{8}\right\}$, e produtos $a b=a^{k}=0, a^{i} a^{j}=a^{i+j}$, para todo $k \geq 9$ e $i, j \geq 1$, e (4.14), então $\mathcal{B}$ é de p.a. e nilíndice 9 .

Prova: Seja $\mathcal{B}$ como no enunciado do teorema. Então $\overline{\mathcal{B}}=\mathcal{B} / A_{a}^{8}=$ $\left\langle\bar{b}, \bar{a}, \bar{a}^{2}, \bar{a}^{3}, \bar{a}^{4}, \bar{a}^{5}, \bar{a}^{6}, \bar{a}^{7}\right\rangle$ e pelo Teorema 4.40 , temos que $\bar{b}^{2}=\alpha_{6} \bar{a}^{6}+\alpha_{7} \bar{a}^{7}$ e $\bar{a}^{2} \bar{b}=\gamma_{5} \bar{a}^{5}+\gamma_{6} \bar{a}^{6}+\gamma_{7} \bar{a}^{7}, \log \mathrm{O}$

$$
b^{2}=\alpha_{6} a^{6}+\alpha_{7} a^{7}+\alpha_{8} a^{8} \quad \text { e } \quad a^{2} b=\gamma_{5} a^{5}+\gamma_{6} a^{6}+\gamma_{7} a^{7}+\gamma_{8} a^{8},
$$

portanto, por (4.1) a (4.5) temos que

$$
\begin{aligned}
a^{3} b & =-\gamma_{5} a^{6}-\gamma_{6} a^{7}-\gamma_{7} a^{8} \\
a^{4} b & =\gamma_{5} a^{7}+\gamma_{6} a^{8} \\
a^{5} b & =-\frac{5}{3} \gamma_{5} a^{8}
\end{aligned}
$$


$a^{6} b=a^{7} b=0 \mathrm{e}$

$$
a^{2} b^{2}=\alpha_{6} a^{8}=\frac{1}{2}\left(\alpha_{6}-\frac{5}{3} \gamma_{5}^{2}\right) a^{8},
$$

de onde $\alpha_{6}=-\frac{5}{3} \gamma_{5}^{2}$, isto é,

$$
b^{2}=-\frac{5}{3} \gamma_{5}^{2} a^{6}+\alpha_{7} a^{7}+\alpha_{8} a^{8} .
$$

Seja

$$
x=b+a+a^{2} .
$$

Então

$$
\begin{aligned}
x^{2}= & a^{2}+2 a^{3}+a^{4}+2 \gamma_{5} a^{5}+\left(-\frac{5}{3} \gamma_{5}^{2}+2 \gamma_{6}\right) a^{6}+\left(2 \gamma_{7}+\alpha_{7}\right) a^{7} \\
& +\left(2 \gamma_{8}+\alpha_{8}\right) a^{8}, \\
x^{3}= & a^{3}+3 a^{4}+\left(3+\gamma_{5}\right) a^{5}+\left(1+\gamma_{6}\right) a^{6}+\left(3 \gamma_{5}-\frac{5}{3} \gamma_{5}^{2}+\gamma_{7}\right) a^{7} \\
& +\left(-5 \gamma_{5}^{2}+3 \gamma_{6}+\gamma_{8}+\alpha_{7}\right) a^{8}, \\
x^{4}= & a^{4}+4 a^{5}+6 a^{6}+\left(4+4 \gamma_{5}\right) a^{7}+\left(1-2 \gamma_{5}-\frac{10}{3} \gamma_{5}^{2}+4 \gamma_{6}\right) a^{8}
\end{aligned}
$$

$\mathrm{e}$

$$
x^{2} x^{2}=a^{4}+4 a^{5}+6 a^{6}+\left(4+4 \gamma_{5}\right) a^{7}+\left(1+8 \gamma_{5}-\frac{10}{3} \gamma_{5}^{2}+4 \gamma_{6}\right) a^{8},
$$

e como $\mathcal{B}$ é de p.a. temos que

$$
0=x^{4}-x^{2} x^{2}=-10 \gamma_{5} a^{8},
$$

do que segue que $\gamma_{5}=0$, portanto $b^{2}=\alpha_{7} a^{7}+\alpha_{8} a^{8}, a^{2} b=\gamma_{6} a^{6}+\gamma_{7} a^{7}+\gamma_{8} a^{8}$, $a^{3} b=-\gamma_{6} a^{7}-\gamma_{7} a^{8}, a^{4} b=\gamma_{6} a^{8}$ e $a^{t} b=0$ para $t \geq 5$.

Seja $\mathcal{B}$ uma nilálgebra comutativa, com base $\left\{b, a, a^{2}, \ldots, a^{8}\right\}$ e produtos $a b=a^{k}=0, a^{i} a^{j}=a^{i+j}$, para todo $k \geq 9$ e $i, j \geq 1$, e (4.14). Então para $x=\lambda b+\sum_{i=1}^{8} \lambda_{i} a^{i}$, temos que

$$
\begin{aligned}
x^{2}= & \lambda_{1}^{2} a^{2}+2 \lambda_{1} \lambda_{2} a^{3}+\left(2 \lambda_{1} \lambda_{3}+\lambda_{2}^{2}\right) a^{4}+\left(2 \lambda_{1} \lambda_{4}+2 \lambda_{2} \lambda_{3}\right) a^{5}+ \\
& \left(2 \lambda \lambda_{2} \gamma_{6}+2 \lambda_{1} \lambda_{5}+2 \lambda_{2} \lambda_{4}+\lambda_{3}^{2}\right) a^{6}+\left(\lambda^{2} \alpha_{7}+2 \lambda \lambda_{2} \gamma_{7}-2 \lambda \lambda_{3} \gamma_{6}\right. \\
& \left.+2 \lambda_{1} \lambda_{6}+2 \lambda_{2} \lambda_{5}+2 \lambda_{3} \lambda_{4}\right) a^{7}+\left(\lambda^{2} \alpha_{8}+2 \lambda \lambda_{2} \gamma_{8}-2 \lambda \lambda_{3} \gamma_{7}+2 \lambda \lambda_{4} \gamma_{6}\right. \\
& \left.+2 \lambda_{1} \lambda_{7}+2 \lambda_{2} \lambda_{6}+2 \lambda_{3} \lambda_{5}+\lambda_{4}^{2}\right) a^{8}
\end{aligned}
$$




$$
\begin{aligned}
x^{3}= & \lambda_{1}^{3} a^{3}+3 \lambda_{1}^{2} \lambda_{2} a^{4}+\left(3 \lambda_{3} \lambda_{1}^{2}+3 \lambda_{1} \lambda_{2}^{2}\right) a^{5}+\left(\lambda \lambda_{1}^{2} \gamma_{6}+3 \lambda_{1}^{2} \lambda_{4}+6 \lambda_{1} \lambda_{2} \lambda_{3}\right. \\
& \left.+\lambda_{2}^{3}\right) a^{6}+\left(\lambda \lambda_{1}^{2} \gamma_{7}+3 \lambda_{1}^{2} \lambda_{5}+6 \lambda_{1} \lambda_{2} \lambda_{4}+3 \lambda_{1} \lambda_{3}^{2}+3 \lambda_{2}^{2} \lambda_{3}\right) a^{7}+\left(\lambda \lambda_{1}^{2} \gamma_{8}\right. \\
& +3 \lambda \lambda_{2}^{2} \gamma_{6}+\lambda_{1} \lambda^{2} \alpha_{7}+3 \lambda_{1}^{2} \lambda_{6}+6 \lambda_{1} \lambda_{2} \lambda_{5}+6 \lambda_{1} \lambda_{3} \lambda_{4}+3 \lambda_{2}^{2} \lambda_{4}+ \\
& \left.3 \lambda_{2} \lambda_{3}^{2}\right) a^{8} \\
x^{4}= & \lambda_{1}^{4} a^{4}+4 \lambda_{1}^{3} \lambda_{2} a^{5}+\left(4 \lambda_{1}^{3} \lambda_{3}+6 \lambda_{1}^{2} \lambda_{2}^{2}\right) a^{6}+\left(4 \lambda_{1}^{3} \lambda_{4}+12 \lambda_{1}^{2} \lambda_{2} \lambda_{3}+\right. \\
& \left.4 \lambda_{1} \lambda_{2}^{3}\right) a^{7}+\left(4 \lambda \lambda_{1}^{2} \lambda_{2} \gamma_{6}+4 \lambda_{1}^{3} \lambda_{5}+12 \lambda_{1}^{2} \lambda_{2} \lambda_{4}+6 \lambda_{1}^{2} \lambda_{3}^{2}+12 \lambda_{1} \lambda_{2}^{2} \lambda_{3}\right. \\
& \left.+\lambda_{2}^{4}\right) a^{8}, \\
x^{5}= & \lambda_{1}^{5} a^{5}+5 \lambda_{1}^{4} \lambda_{2} a^{6}+\left(5 \lambda_{1}^{4} \lambda_{3}+10 \lambda_{1}^{3} \lambda_{2}^{2}\right) a^{7}+\left(\lambda \lambda_{1}^{4} \gamma_{6}+5 \lambda_{1}^{4} \lambda_{4}+\right. \\
& \left.20 \lambda_{1}^{3} \lambda_{2} \lambda_{3}+10 \lambda_{1}^{2} \lambda_{2}^{3}\right) a^{8}, \\
x^{6}= & \lambda_{1}^{6} a^{6}+6 \lambda_{1}^{5} \lambda_{2} a^{7}+\left(6 \lambda_{1}^{5} \lambda_{3}+15 \lambda_{1}^{4} \lambda_{2}^{2}\right) a^{8}, \\
x^{7}= & \lambda_{1}^{7} a^{7}+7 \lambda_{1}^{6} \lambda_{2} a^{8}, \\
x^{8}= & \lambda_{1}^{8} a^{8}, \\
x^{9}= & 0, \\
x^{2} x^{2}= & \lambda_{1}^{4} a^{4}+4 \lambda_{1}^{3} \lambda_{2} a^{5}+\left(4 \lambda_{1}^{3} \lambda_{3}+6 \lambda_{1}^{2} \lambda_{2}^{2}\right) a^{6}+\left(4 \lambda_{1}^{3} \lambda_{4}+12 \lambda_{1}^{2} \lambda_{2} \lambda_{3}+\right. \\
& \left.4 \lambda_{1} \lambda_{2}^{3}\right) a^{7}+\left(4 \lambda \lambda_{1}^{2} \lambda_{2} \gamma_{6}+4 \lambda_{1}^{3} \lambda_{5}+12 \lambda_{1}^{2} \lambda_{2} \lambda_{4}+6 \lambda_{1}^{2} \lambda_{3}^{2}+12 \lambda_{1} \lambda_{2}^{2} \lambda_{3}\right. \\
& \left.+\lambda_{2}^{4}\right) a^{8},
\end{aligned}
$$

assim $x^{4}=x^{2} x^{2}$ e podemos concluir que $\mathcal{B}$ é de p.a. e tem nilíndice 9.

Teorema 4.47 Seja n maior ou igual que 8. Se $\mathcal{B}$ é uma nilálgebra comutativa de p.a. de dimensão e nilíndice $n$ e $(a, b)$ um elemento em $\mathcal{P}(\mathcal{B})$, então para a base $\left\{b, a, a^{2}, \ldots, a^{n-1}\right\}$ de $\mathcal{B}$ os produtos não triviais são dados por:

$$
\begin{aligned}
b^{2} & =\alpha_{n-2} a^{n-2}+\alpha_{n-1} a^{n-1}, \\
a^{2} b & =\gamma_{n-3} a^{n-3}+\gamma_{n-2} a^{n-2}+\gamma_{n-1} a^{n-1}, \\
a^{3} b & =-\gamma_{n-3} a^{n-2}-\gamma_{n-2} a^{n-1}, \\
a^{4} b & =\gamma_{n-3} a^{n-1} .
\end{aligned}
$$

Reciprocamente, se $\mathcal{B}$ é uma nilálgebra comutativa, com base $\left\{b, a, a^{2}, \ldots, a^{n-1}\right\}$, 
e produtos $a b=a^{k}=0, a^{i} a^{j}=a^{i+j}$, para $k \geq n \quad e \quad i, j \geq 1$, e (4.16), então $\mathcal{B}$ é de p.a. e nilíndice $n$.

Prova: Demonstraremos a primeira parte do teorema por indução sobre $n$. Para $n$ igual a 8 e 9, o resultado é válido pelos Teoremas 4.40 e 4.46.

Suponhamos, por hipótese de indução, que para $8 \leq k \leq n-1$, os produtos não nulos na base $\left\{b, a, a^{2}, \ldots, a^{k}\right\}$ são dados por:

$$
\begin{aligned}
b^{2} & =\alpha_{k-2} a^{k-2}+\alpha_{k-1} a^{k-1}, \\
a^{2} b & =\gamma_{k-3} a^{n-3}+\gamma_{k-2} a^{k-2}+\gamma_{k-1} a^{k-1}, \\
a^{3} b & =-\gamma_{k-3} a^{k-2}-\gamma_{k-2} a^{k-1}, \\
a^{4} b & =\gamma_{k-3} a^{k-1},
\end{aligned}
$$

e demonstremos para $k=n$.

Seja $\mathcal{B}$ uma nilálgebra comutativa de p.a. de dimensão e nilíndice $n$ e base $\left\{b, a, a^{2}, \cdots, a^{n-1}\right\}$ e produtos não triviais dados por (4.16). Uma vez que $A_{a}^{n-1}=\left\langle a^{n-1}\right\rangle$ é um ideal de $\mathcal{B}$, temos que $\overline{\mathcal{B}}=\mathcal{B} / A_{a}^{n-1}=$ $\left\{\bar{b}, \bar{a}, \bar{a}^{2}, \cdots, \bar{a}^{n-2}\right\}$. Pela hipótese de indução,

$$
\bar{b}^{2}=\alpha_{n-3} \bar{a}^{n-3}+\alpha_{n-2} \bar{a}^{n-2}
$$

$\mathrm{e}$

$$
\bar{a}^{2} \bar{b}=\gamma_{n-4} \bar{a}^{n-4}+\gamma_{n-3} \bar{a}^{n-3}+\gamma_{n-2} \bar{a}^{n-2}
$$

$\log 0$

$$
b^{2}=\alpha_{n-3} a^{n-3}+\alpha_{n-2} a^{n-2}+\alpha_{n-1} a^{n-1}
$$

$\mathrm{e}$

$$
a^{2} b=\gamma_{n-4} a^{n-4}+\gamma_{n-3} a^{n-3}+\gamma_{n-2} a^{n-2}+\gamma_{n-1} a^{n-1},
$$

e utilizando as igualdades (4.1) a (4.5) obtemos que

$$
a^{3} b=-\gamma_{n-4} a^{n-3}-\gamma_{n-3} a^{n-2}+\gamma_{n-2} a^{n-1},
$$




$$
\begin{aligned}
a^{4} b= & \frac{1}{3}\left[3\left(\gamma_{n-4} a^{n-2}+\gamma_{n-3} a^{n-1}\right)-2\left(-\gamma_{n-4} a^{n-2}-\gamma_{n-3} a^{n-1}\right)\right. \\
& \left.-2\left(\gamma_{n-4} a^{n-2}+\gamma_{n-3} a^{n-1}\right)\right] \\
= & \gamma_{n-4} a^{n-2}+\gamma_{n-3} a^{n-1}, \\
a^{5} b= & \frac{1}{3}\left[4\left(-\gamma_{n-4} a^{n-1}\right)-\left(\gamma_{n-4} a^{n-1}\right)-2\left(-\gamma_{n-4} a^{n-1}\right)\right. \\
& \left.-2\left(\gamma_{n-4} a^{n-1}\right)\right] \\
= & -\frac{5}{3} \gamma_{n-4} a^{n-1},
\end{aligned}
$$

e para $r \geq 4$, temos que $a^{2}\left(a^{r} b\right) \in A_{a}^{2} A_{a}^{n-2}=A_{a}^{n}=\{0\}, \quad a^{r}\left(a^{2} b\right) \in A_{a}^{4} A_{a}^{n-4}=$ $A_{a}^{n}=\{0\}, a\left(a^{r+1} b\right) \in A_{a} A_{a}^{n-1}=A_{a}^{n}=\{0\}$ е $a\left(a\left(a^{r} b\right)\right) \in A_{a} A_{a} A_{a}^{n-2}=A_{a}^{n}=$ $\{0\}$, portanto

$$
a^{r+2} b=\frac{1}{3}\left[4 a^{2}\left(a^{r} b\right)-a^{r}\left(a^{2} b\right)-2 a\left(a^{r+1} b\right)-2 a\left(a\left(a^{r} b\right)\right)\right]=0,
$$

para $r \geq 4$, assim

$$
a^{t} b=0 \quad \text { para } \quad t \geq 6 .
$$

Temos que

$$
a^{2} b^{2}=\alpha_{n-3} a^{n-1}
$$

e por (4.1),

$$
\begin{aligned}
a^{2} b^{2} & =\frac{1}{2}\left[\alpha_{n-3} a^{n-1}+\gamma_{n-4} a^{n-4} b+\gamma_{n-3} a^{n-3} b+\gamma_{n-2} a^{n-2} b+\gamma_{n-1} a^{n-1} b\right], \\
& =\frac{1}{2} \alpha_{n-3} a^{n-1},
\end{aligned}
$$

pois para $n \geq 10$ temos que $a^{n-i} \geq 6$ e portanto $a^{n-i} b=0$, para $1 \leq i \leq 4$, de onde $\alpha_{n-3}=0$. Assim

$$
b^{2}=\alpha_{n-2} a^{n-2}+\alpha_{n-1} a^{n-1} .
$$

Seja

$$
x=b+a+a^{2} .
$$

Então 


$$
\begin{aligned}
x^{2}= & a^{2}+2 a^{3}+a^{4}+2 \gamma_{n-4} a^{n-4}+2 \gamma_{n-3} a^{n-3}+\left(2 \gamma_{n-2}+\alpha_{n-2}\right) a^{n-2} \\
& +\left(2 \gamma_{n-1}+\alpha_{n-1}\right) a^{n-1}, \\
x^{3}= & a^{3}+3 a^{4}+3 a^{5}+a^{6}+\gamma_{n-4} a^{n-4}+\gamma_{n-3} a^{n-3}+\left(\gamma_{n-2}+3 \gamma_{n-4}\right) a^{n-2} \\
& +\left(\gamma_{n-1}+3 \gamma_{n-3}+\alpha_{n-2}\right) a^{n-1} \\
x^{4}= & a^{4}+4 a^{5}+6 a^{6}+4 a^{7}+a^{8}+4 \gamma_{n-4} a^{n-2}+\left(4 \gamma_{n-3}-2 \gamma_{n-4}\right) a^{n-1}
\end{aligned}
$$

e

$$
x^{2} x^{2}=a^{4}+4 a^{5}+6 a^{6}+4 a^{7}+a^{8}+4 \gamma_{n-4} a^{n-2}+\left(4 \gamma_{n-3}+8 \gamma_{n-4}\right) a^{n-1},
$$

$\log 0$

$$
0=x^{4}-x^{2} x^{2}=-10 \gamma_{n-4} a^{n-1},
$$

pois $\mathcal{B}$ é de p.a., do que segue que $\gamma_{n-4}=0$, portanto $b^{2}=\alpha_{n-2} a^{n-2}+$ $\alpha_{n-1} a^{n-1}, a^{2} b=\gamma_{n-3} a^{n-3}+\gamma_{n-2} a^{n-2}+\gamma_{n-1} a^{n-1}, a^{3} b=-\gamma_{n-3} a^{n-2}-$ $\gamma_{n-2} a^{n-1}, a^{4} b=\gamma_{n-3} a^{n-1}$ e $a^{t} b=0$ para $t \geq 5$.

Para demonstrar a outra parte do teorema, seja $\mathcal{B}$ uma nilálgebra comutativa com base $\left\{b, a, \ldots, a^{n-1}\right\}$ e produtos $a b=a^{k}=0, a^{i} a^{j}=a^{i+j}$, para $k \geq$ $n$ e $i, j \geq 1$, e (4.16). Mostraremos primeiro que $\mathcal{B}$ é de p.a. Para isto, seja $x=\lambda b+p(a) \operatorname{com} p(a)=\sum_{i=1}^{n-1} \lambda_{i} a^{i}$. Então

$$
\begin{aligned}
x^{2}= & \lambda^{2} b^{2}+2 \lambda b \cdot p(a)+p^{2}(a), \\
x^{3}= & \lambda^{3} b^{3}+2 \lambda^{2} b(b \cdot p(a))+\lambda b \cdot p^{2}(a)+\lambda^{2} b^{2} \cdot p(a)+2 \lambda p(a)(b \cdot p(a)) \\
& +p^{3}(a), \\
x^{4}= & \lambda^{4} b^{4}+2 \lambda^{3} b(b(b \cdot p(a)))+\lambda^{2} b\left(b \cdot p^{2}(a)\right)+\lambda^{3} b\left(b^{2} \cdot p(a)\right)+ \\
& 2 \lambda^{2} b(p(a)(b \cdot p(a)))+\lambda b \cdot p^{3}(a)+\lambda^{3} p(a) \cdot b^{3}+2 \lambda^{2} p(a)(b(b \cdot p(a)))+ \\
& \lambda p(a)\left(b \cdot p^{2}(a)\right)+\lambda^{2} p(a)\left(b^{2} \cdot p(a)\right)+2 \lambda p(a)\left(p(a)(b \cdot p(a))+p^{4}(a),\right. \\
x^{2} x^{2}= & \lambda^{4} b^{2} \cdot b^{2}+4 \lambda^{3} b^{2}(b \cdot p(a))+2 \lambda^{2} b^{2} \cdot p^{2}(a)+4 \lambda^{2}(b \cdot p(a))(b \cdot p(a)) \\
& +4 \lambda p^{2}(a)(b \cdot p(a))+p^{2}(a) \cdot p^{2}(a) .
\end{aligned}
$$


Provaremos que $x^{4}-x^{2} x^{2}=0$, verificando as seguintes igualdades:

$$
\begin{aligned}
4 b^{2}(b \cdot p(a))= & 2 b(b(b \cdot p(a)))+b\left(b^{2} \cdot p(a)\right)+b^{3} \cdot p(a), \\
4 p^{2}(a)(b \cdot p(a))= & b \cdot p^{3}(a)+p(a)\left(b \cdot p^{2}(a)\right)+ \\
& 2 p(a)(p(a)(b \cdot p(a))), \\
2 b^{2} \cdot p^{2}(a)+4(b \cdot p(a))^{2}= & b\left(b \cdot p^{2}(a)\right)+2 b(p(a)(b \cdot p(a)))+ \\
& 2 p(a)(b(b \cdot p(a)))+p(a)\left(b^{2} \cdot p(a)\right) .
\end{aligned}
$$

Uma vez que,

$$
p(a)=\lambda_{1} a+\lambda_{2} a^{2}+\lambda_{3} a^{3}+\lambda_{4} a^{4}+\sum_{i=5}^{n-1} \lambda_{i} a^{i},
$$

temos que

$$
\begin{aligned}
b \cdot p(a)= & \lambda_{2} b a^{2}+\lambda_{3} b a^{3}+\lambda_{4} b a^{4} \\
= & \lambda_{2}\left(\gamma_{n-3} a^{n-3}+\gamma_{n-2} a^{n-2}+\gamma_{n-1} a^{n-1}\right)+\lambda_{3}\left(-\gamma_{n-3} a^{n-2}-\right. \\
& \left.\gamma_{n-2} a^{n-1}\right)+\lambda_{4} \gamma_{n-3} a^{n-1},
\end{aligned}
$$

isto é,

$$
\begin{aligned}
b \cdot p(a)= & \lambda_{2} \gamma_{n-3} a^{n-3}+\left(\lambda_{2} \gamma_{n-2}-\lambda_{3} \gamma_{n-3}\right) a^{n-2}+ \\
& \left(\lambda_{2} \gamma_{n-1}-\lambda_{3} \gamma_{n-2}+\lambda_{4} \gamma_{n-3}\right) a^{n-1} \in A_{a}^{n-3} .
\end{aligned}
$$

Portanto $b(b \cdot p(a))=0$, e assim

$$
b^{2}(b \cdot p(a))=b(b(b \cdot p(a)))=0 .
$$

Por outro lado, temos que, $b^{3}=b b^{2} \in b A_{a}^{n-2}=\{0\}$ e

$$
b^{2} \cdot p(a)=\left(\alpha_{n-2} a^{n-2}+\alpha_{n-1} a^{n-1}\right) \sum_{i=1}^{n-1} \lambda_{i} a^{i}=\lambda_{1} \alpha_{n-2} a^{n-1} .
$$

Em consequência,

$$
b\left(b^{2} \cdot p(a)\right)=b^{3} \cdot p(a)=0
$$


o que verifica (4.17).

Temos que

$$
\begin{aligned}
p^{2}(a)= & \lambda_{1}^{2} a^{2}+2 \lambda_{1} \lambda_{2} a^{3}+\left(2 \lambda_{1} \lambda_{3}+\lambda_{2}^{2}\right) a^{4}+ \\
& \sum_{i=3}^{n-1} \lambda_{i}^{2} a^{2 i}+2 \sum_{i=1}^{n-2} \sum_{\substack{j=i+1 \\
(i, j) \neq(1,2),(1,3)}}^{n-1} \lambda_{i} \lambda_{j} a^{i+j}, \\
p^{3}(a)= & \lambda_{1}^{3} a^{3}+3 \lambda_{1}^{2} \lambda_{2} a^{4}+\sum_{i=5}^{n-1} \theta_{i} a^{i},
\end{aligned}
$$

$\log 0$

$b \cdot p^{3}(a)=\lambda_{1}^{3} b a^{3}+3 \lambda_{1}^{2} \lambda_{2} b a^{4}=-\lambda_{1}^{3}\left(\gamma_{n-3} a^{n-2}+\gamma_{n-2} a^{n-1}\right)+3 \lambda_{1}^{2} \lambda_{2} \gamma_{n-3} a^{n-1}$, pois $b a^{i}=0$, para $i \geq 5$. Assim,

$$
b \cdot p^{3}(a)=-\lambda_{1}^{3} \gamma_{n-3} a^{n-2}+\left(-\lambda_{1}^{3} \gamma_{n-2}+3 \lambda_{1}^{2} \lambda_{2} \gamma_{n-3}\right) a^{n-1} .
$$

Agora

$$
\begin{aligned}
b \cdot p^{2}(a)= & \lambda_{1}^{2} b a^{2}+2 \lambda_{1} \lambda_{2} b a^{3}+\left(2 \lambda_{1} \lambda_{3}+\lambda_{2}^{2}\right) b a^{4} \\
= & \lambda_{1}^{2} \gamma_{n-3} a^{n-3}+\lambda_{1}\left(\lambda_{1} \gamma_{n-2}-2 \lambda_{2} \gamma_{n-3}\right) a^{n-2}+\left(\lambda_{1}^{2} \gamma_{n-1}+\lambda_{2}^{2} \gamma_{n-3}+\right. \\
& \left.2 \lambda_{1} \lambda_{3} \gamma_{n-3}-2 \lambda_{1} \lambda_{2} \gamma_{n-2}\right) a^{n-1} \in A_{a}^{n-3},
\end{aligned}
$$

do que segue que

$p(a)\left(b \cdot p^{2}(a)\right)=\lambda_{1}^{3} \gamma_{n-3} a^{n-2}+\lambda_{2} \lambda_{1}^{2} \gamma_{n-3} a^{n-1}+\lambda_{1}^{2}\left(\lambda_{1} \gamma_{n-2}-2 \lambda_{2} \gamma_{n-3}\right) a^{n-1}$,

isto é,

$$
p(a)\left(b \cdot p^{2}(a)\right)=\lambda_{1}^{3} \gamma_{n-3} a^{n-2}+\lambda_{1}^{2}\left(-\lambda_{2} \gamma_{n-3}+\lambda_{1} \gamma_{n-2}\right) a^{n-1} .
$$

Por (4.20), temos que

$$
\begin{aligned}
p^{2}(a)(b \cdot p(a)) & =\lambda_{1}^{2} \lambda_{2} \gamma_{n-3} a^{n-1}, \\
p(a)(b \cdot p(a)) & =\lambda_{1} \lambda_{2} \gamma_{n-3} a^{n-2}+\lambda_{1}\left(\lambda_{2} \gamma_{n-2}-\lambda_{3} \gamma_{n-3}\right) a^{n-1}+\lambda_{2}^{2} \gamma_{n-3} a^{n-1} \\
& =\lambda_{1} \lambda_{2} \gamma_{n-3} a^{n-2}+\left(\lambda_{2}^{2} \gamma_{n-3}+\lambda_{1} \lambda_{2} \gamma_{n-2}-\lambda_{1} \lambda_{3} \gamma_{n-3}\right) a^{n-1},
\end{aligned}
$$


$\log 0$

$$
p^{2}(a)(b \cdot p(a))=p(a)(p(a)(b \cdot p(a)))=\lambda_{1}^{2} \lambda_{2} \gamma_{n-3} a^{n-1} .
$$

Assim, temos que

$$
\begin{aligned}
b \cdot & p^{3}(a)+p(a)\left(b \cdot p^{2}(a)\right)+2 p(a)(p(a)(b \cdot p(a))) \\
= & -\lambda_{1}^{3} \gamma_{n-3} a^{n-2}+\left(-\lambda_{1}^{3} \gamma_{n-2}+3 \lambda_{1}^{2} \lambda_{2} \gamma_{n-3}\right) a^{n-1}+\lambda_{1}^{3} \gamma_{n-3} a^{n-2}+ \\
& \lambda_{1}^{2}\left(-\lambda_{2} \gamma_{n-3}+\lambda_{1} \gamma_{n-2}\right) a^{n-1}+2 \lambda_{1}^{2} \lambda_{2} \gamma_{n-3} a^{n-1} \\
= & 4 \lambda_{1}^{2} \lambda_{2} \gamma_{n-3} a^{n-1} \\
= & 4 p^{2}(a) \cdot b \cdot p(a),
\end{aligned}
$$

verificando (4.18).

Por $(4.20)$ e $(4.21)$, temos que $b \cdot p(a), b \cdot p^{2}(a) \in A_{a}^{n-3}, \operatorname{logo}(b \cdot p(a))^{2} \in$ $A_{a}^{2(n-3)}=\{0\}$ e $b\left(b \cdot p^{2}(a)\right) \in b A_{a}^{n-3}=\{0\}$, e uma vez que, $b^{2} \cdot p^{2}(a) \in$ $A_{a}^{n-2} A_{a}^{2}=\{0\}$, podemos concluir que

$$
(b \cdot p(a))^{2}=b\left(b \cdot p^{2}(a)\right)=b^{2} \cdot p^{2}(a)=0 .
$$

Por outro lado, segue de (4.20), que $b(b \cdot p(a)) \in b A_{a}^{n-3}=\{0\}$ e $p(a)(b \cdot p(a)) \in$ $A_{a}^{n-2}$, portanto $p(a)(b(b \cdot p(a)))=0$ e $b(p(a)(b \cdot p(a))) \in b A_{a}^{n-2}=\{0\}$. Finalmente, temos que $p(a)\left(b^{2} \cdot p(a)\right) \in A_{a} A_{a}^{n-2} A_{a}=A_{a}^{n}=\{0\}$. Assim

$$
p(a)(b(b \cdot p(a)))=b(p(a)(b \cdot p(a)))=p(a)\left(b^{2} \cdot p(a)\right)=0,
$$

o que nos verifica (4.19).

Para concluirmos que $x^{4}=x^{2} x^{2}$, resta mostrar que $b^{4}=b^{2} b^{2}$ e que $p^{4}(a)=p^{2}(a) p^{2}(a)$. O segundo fato é imediato da tabela de multiplicação para os $a^{i}$, em quanto ao primeiro, temos que, $b^{2} b^{2} \in A_{a}^{2(n-2)}=\{0\}$, e uma vez que mostramos que $b^{3}=0$, segue que $b^{4}=0$. Assim podemos concluir, de todo o anterior, que $\mathcal{B}$ é de p.a.

Passemos agora a demonstrar que $\mathcal{B}$ tem nilíndice $n-1$. Seja $x=\lambda b+$ $\sum_{i=1}^{n-1} \lambda_{i} a^{i}$. Mostraremos que para $n \geq 9$ e $k \geq 2$, cumpre-se que:

$$
x^{k}=\lambda_{1}^{k} a^{k}+k \lambda_{1}^{k-1} \lambda_{2} a^{k+1}+\left[k \lambda_{3} \lambda_{1}^{k-1}+\frac{k(k-1)}{2} \lambda_{2}^{2} \lambda_{1}^{k-2}\right] a^{k+2}+\sum_{i=k+3}^{n-1} \theta_{i} a^{i},
$$


sendo que $\sum_{i=k+3}^{n-1} \theta_{i} a^{i}=0$ para $k \geq n-3$.

Demonstraremos a igualdade anterior por indução sobre $n$. Para $n=9$, temos por (4.15) que:

$$
\begin{aligned}
x^{2} & =\lambda_{1}^{2} a^{2}+2 \lambda_{1} \lambda_{2} a^{3}+\left(2 \lambda_{1} \lambda_{3}+\lambda_{2}^{2}\right) a^{4}+\sum_{i=5}^{8} \theta_{2, i} a^{i}, \\
x^{3} & =\lambda_{1}^{3} a^{3}+3 \lambda_{1}^{2} \lambda_{2} a^{4}+\left(3 \lambda_{3} \lambda_{1}^{2}+3 \lambda_{2}^{2} \lambda_{1}\right) a^{5}+\sum_{i=6}^{8} \theta_{3, i} a^{i}, \\
x^{4} & =\lambda_{1}^{4} a^{4}+4 \lambda_{1}^{3} \lambda_{2} a^{5}+\left(4 \lambda_{3} \lambda_{1}^{3}+6 \lambda_{2}^{2} \lambda_{1}^{2}\right) a^{6}+\sum_{i=7}^{8} \theta_{4, i} a^{i}, \\
x^{5} & =\lambda_{1}^{5} a^{5}+5 \lambda_{1}^{4} \lambda_{2} a^{6}+\left(5 \lambda_{3} \lambda_{1}^{4}+10 \lambda_{2}^{2} \lambda_{1}^{3}\right) a^{7}+\theta a^{8} \\
x^{6} & =\lambda_{1}^{6} a^{6}+6 \lambda_{1}^{5} \lambda_{2} a^{7}+\left(6 \lambda_{3} \lambda_{1}^{5}+15 \lambda_{2}^{2} \lambda_{1}^{4}\right) a^{8} \\
x^{7} & =\lambda_{1}^{7} a^{7}+7 \lambda_{1}^{6} \lambda_{2} a^{8}, \\
x^{8} & =\lambda_{1}^{8} a^{8} .
\end{aligned}
$$

o que verifica (4.22) para dimensão 9.

Suponhamos por hipótese de indução que

$$
x^{k}=\lambda_{1}^{k} a^{k}+k \lambda_{1}^{k-1} \lambda_{2} a^{k+1}+\left[k \lambda_{3} \lambda_{1}^{k-1}+\frac{k(k-1)}{2} \lambda_{2}^{2} \lambda_{1}^{k-2}\right] a^{k+2}+\sum_{i=k+3}^{m-1} \theta_{i} a^{i},
$$

para $9 \leq m \leq n-1$ e demonstremos a igualdade para $m=n$.

Seja $\mathcal{B}$ uma nilálgebra comutativa, com base $\left\{b, a, a^{2}, \ldots, a^{n-1}\right\}$, e produtos $a b=a^{k}=0, a^{i} a^{j}=a^{i+j}, \quad$ para $k \geq n$ e $i, j \geq 1$, e (4.16). Se $x \in \mathcal{B}$, então lembrando que $\left\langle a^{n-1}\right\rangle$ é um ideal de $\mathcal{B}$, temos que $\bar{x} \in \overline{\mathcal{B}}=\mathcal{B} /\left\langle a^{n-1}\right\rangle$ é da forma $\bar{x}=\lambda \bar{b}+\lambda_{1} \bar{a}+\lambda_{2} \bar{a}^{2}+\cdots+\lambda_{n-2} \bar{a}^{n-2}$, logo pela hipótese de indução temos que

$$
\bar{x}^{k}=\lambda_{1}^{k} \bar{a}^{k}+k \lambda_{1}^{k-1} \lambda_{2} \bar{a}^{k+1}+\left[k \lambda_{3} \lambda_{1}^{k-1}+\frac{k(k-1)}{2} \lambda_{2}^{2} \lambda_{1}^{k-2}\right] \bar{a}^{k+2}+\sum_{i=k+3}^{n-2} \theta_{i} \bar{a}^{i} .
$$


Portanto

$$
\begin{aligned}
x^{k}= & \lambda_{1}^{k} a^{k}+k \lambda_{1}^{k-1} \lambda_{2} a^{k+1}+\left[k \lambda_{3} \lambda_{1}^{k-1}+\frac{k(k-1)}{2} \lambda_{2}^{2} \lambda_{1}^{k-2}\right] a^{k+2}+\sum_{i=k+3}^{n-2} \theta_{i} a^{i} \\
& +\theta a^{n-1}
\end{aligned}
$$

$\mathrm{e}$

$$
\begin{aligned}
& x^{k+1}= \lambda_{1}^{k} x a^{k}+k \lambda_{1}^{k-1} \lambda_{2} x a^{k+1}+\left[k \lambda_{3} \lambda_{1}^{k-1}+\frac{k(k-1)}{2} \lambda_{2}^{2} \lambda_{1}^{k-2}\right] x a^{k+2} \\
&+\sum_{i=k+3}^{n-2} \theta_{i} x a^{i}+\theta x a^{n-1} \\
&= \lambda_{1}^{k} \lambda b a^{k}+\lambda_{1}^{k+1} a^{k+1}+\lambda_{1}^{k} \lambda_{2} a^{k+2}+\lambda_{1}^{k} \lambda_{3} a^{k+3}+\lambda_{1}^{k} \sum_{i=4}^{n-1} \lambda_{i} a^{i+k}+ \\
& k \lambda_{1}^{k-1} \lambda_{2} \lambda b a^{k+1}+k \lambda_{1}^{k} \lambda_{2} a^{k+2}+k \lambda_{1}^{k-1} \lambda_{2}^{2} a^{k+3}+k \lambda_{1}^{k-1} \lambda_{2} \sum_{i=3}^{n-1} \lambda_{i} a^{k+1+i} \\
&+\left[k \lambda_{3} \lambda_{1}^{k-1}+\frac{k(k-1)}{2} \lambda_{2}^{2} \lambda_{1}^{k-2}\right] \lambda b a^{k+2}+\left[k \lambda_{3} \lambda_{1}^{k}+\frac{k(k-1)}{2} \lambda_{2}^{2} \lambda_{1}^{k-1}\right] \\
& a^{k+3}+\left[k \lambda_{3} \lambda_{1}^{k-1}+\frac{k(k-1)}{2} \lambda_{2}^{2} \lambda_{1}^{k-2}\right] \sum_{i=2}^{n-1} \lambda_{i} a^{k+2+i}+\sum_{i=k+4}^{n-1} \tilde{\theta}_{i} a^{i} \\
&= \lambda_{1}^{k+1} a^{k+1}+\left(\lambda_{1}^{k} \lambda_{2}+k \lambda_{1}^{k} \lambda_{2}\right) a^{k+2}+\left\{\lambda_{1}^{k} \lambda_{3}+k \lambda_{1}^{k-1} \lambda_{2}^{2}+\left[k \lambda_{3} \lambda_{1}^{k}+\right.\right. \\
&\left.\left.\frac{k(k-1)}{2} \lambda_{2}^{2} \lambda_{1}^{k-1}\right]\right\} a^{k+3}+\sum_{i=k+4}^{n-1} \rho_{i} a^{i} \\
&+\sum_{i=k+4}^{n-1} \rho_{i} a^{i}, \\
& \lambda_{1}^{k+1} a^{k+1}+(k+1) \lambda_{1}^{k} \lambda_{2} a^{k+2}+\left[(k+1) \lambda_{3} \lambda_{1}^{k}+\frac{k(k+1)}{2} \lambda_{2}^{2} \lambda_{1}^{k-1}\right] a^{k+3} \\
&{ }_{k}^{k+1}(k)
\end{aligned}
$$

pois $b a^{k} \in A_{a}^{n-3}$, para $k \geq 2$ e $n \geq 9$, com o qual fica verificada (4.22). Assim para $k=n$, temos que $x^{n}=\lambda_{1}^{n} a^{n}=0$, o que nos mostra que $x^{n}=0$ 
para todo $x \in \mathcal{B}$ e existe $a \in \mathcal{B}$ tal que $a^{n-1} \neq 0$. Portanto podemos concluir que $\mathcal{B}$ tem nilíndice $n$.

Denotaremos por $\mathcal{B}\left(\alpha_{2}, \alpha_{1}, \gamma_{3}, \gamma_{2}, \gamma_{1}\right)$ a álgebra do teorema anterior. Estudemos as classes de isomorfismos de todas as álgebras desta forma. Para tal fixaremos o par $(a, b)$ que determina a base canônica para dita álgebra, quando não houver lugar a a confusão nos referiremos a esta álgebra simplesmente como $\mathcal{B}$.

Sejam $\lambda$ e $\beta$ em $F^{*}$. Então definindo os elemento $a_{1}:=\lambda a$ e $b_{1}:=\beta a$, obtemos o seguinte isomorfismo.

$$
\mathcal{B}\left(\alpha_{2}, \alpha_{1}, \gamma_{3}, \gamma_{2}, \gamma_{1}\right) \cong \mathcal{B}\left(\frac{\beta^{2}}{\lambda^{n-2}} \alpha_{2}, \frac{\beta^{2}}{\lambda^{n-1}} \alpha_{1}, \frac{\beta}{\lambda^{n-5}} \gamma_{3}, \frac{\beta}{\lambda^{n-4}} \gamma_{2}, \frac{\beta}{\lambda^{n-3}} \gamma_{1}\right)
$$

Observamos que se consideramos $\beta=\frac{1}{\gamma_{3}}$ e $\lambda=1$, então

$$
\mathcal{B}\left(\alpha_{2}, \alpha_{1}, \gamma_{3}, \gamma_{2}, \gamma_{1}\right) \cong \mathcal{B}\left(\frac{\alpha_{2}}{\gamma_{3}^{2}}, \frac{\alpha_{1}}{\gamma_{3}^{2}}, 1, \frac{\gamma_{2}}{\gamma_{3}}, \frac{\gamma_{1}}{\gamma_{3}}\right)
$$

Portanto podemos supor, a menos de isomorfismos, que $\gamma_{3}=0$ ou $\gamma_{3}=1$. Achemos então as classes de isomorfismos considerando estes dois casos.

- $\left(\gamma_{3}=1\right.$.) Neste caso temos a álgebra $\mathcal{B}\left(\alpha_{2}, \alpha_{1}, 1, \gamma_{2}, \gamma_{1}\right)$.

Lema 4.48 Sejam $\alpha_{2}, \alpha_{1}, \gamma_{2}$ e $\gamma_{1}$ em F. Então

$$
\mathcal{B}\left(\alpha_{2}, \alpha_{1}, 1, \gamma_{2}, \gamma_{1}\right) \cong \mathcal{B}\left(\alpha_{2}^{\prime}, \alpha_{1}^{\prime}, 1,0,0\right)
$$

Prova: Seja $\mathcal{B}$ a álgebra $\mathcal{B}\left(\alpha_{2}, \alpha_{1}, 1, \gamma_{2}, \gamma_{1}\right)$ em relação a base determinada pelo par $(a, b)$. Definimos os seguintes elementos em $\mathcal{B}$,

$$
\begin{aligned}
a_{1}:= & a+\frac{\gamma_{2}}{n} a^{2}+\left[\frac{\gamma_{1}}{(n-6)}-\frac{\left(n^{2}-n+12\right) \gamma_{2}^{2}}{2 n^{2}(n-6)}\right] a^{3} \\
& +\left[\frac{6 \gamma_{1} \gamma_{2}}{n(n-6)}-\frac{\left(n^{3}-3 n^{2}+26 n-12\right) \gamma_{2}^{3}}{2 n^{3}(n-6)}\right] a^{4}
\end{aligned}
$$


$\mathrm{e}$

$$
b_{1}:=b-\frac{\gamma_{2}}{n} a^{n-4}+\left[\frac{\gamma_{1}}{(n-6)}-\frac{3\left(n^{2}-5 n+8\right) \gamma_{2}^{2}}{2 n^{2}(n-6)}\right] a^{n-3}
$$

Observamos que $a_{1}$ é um elemento de nilíndice máximo, pois por (4.22) $a_{1}^{n-1}=a^{n-1} \neq 0$. Da definição é claro que $b_{1} \notin A_{a_{1}}$, e fazendo um calculo direto obtemos que $a_{1} b_{1}=0$. Portanto $\left(a_{1}, b_{1}\right)$ está em $\mathcal{P}(\mathcal{B})$. Utilizando (4.22) para calcular $a_{1}^{2}, a_{1}^{n-3}, a_{1}^{n-2}$ e $a_{1}^{n-1}$, e calculando os produtos, $b_{1}^{2}$ e $a_{1}^{2} b_{1}$, obtemos que

$$
b_{1}^{2}=\alpha_{2}^{\prime} a_{1}^{n-2}+\alpha_{2}^{\prime} a_{1}^{n-1} \text { e } a_{1}^{2} b_{1}=a_{1}^{n-3},
$$

o que demonstra o lema.

- $\left(\gamma_{3}=0.\right)$ Neste caso temos a álgebra $\mathcal{B}\left(\alpha_{2}, \alpha_{1}, 0, \gamma_{2}, \gamma_{1}\right)$.

Se $\gamma_{2} \neq 0$, então considerando $\beta=\frac{1}{\gamma_{2}}$ e $\lambda=1$, em (4.23) segue que

$$
\mathcal{B}\left(\alpha_{2}, \alpha_{1}, 0, \gamma_{2}, \gamma_{1}\right) \cong \mathcal{B}\left(\alpha_{2}^{\prime}, \alpha_{1}^{\prime}, 0,1, \gamma_{1}^{\prime}\right)
$$

Lema 4.49 Sejam $\alpha_{2}, \alpha_{1}$ e $\gamma_{1}$ em F. Então

$$
\mathcal{B}\left(\alpha_{2}, \alpha_{1}, 0,1, \gamma_{1}\right) \cong \mathcal{B}\left(\alpha_{2}^{\prime}, \alpha_{1}^{\prime}, 0,1,0\right)
$$

Prova: Seja $\mathcal{B}$ a álgebra $\mathcal{B}\left(\alpha_{2}, \alpha_{1}, 0,1, \gamma_{1}\right)$ em relação a base determinada pelo par $(a, b)$. Definimos os seguintes elementos em $\mathcal{B}$,

$$
\begin{aligned}
a_{1} & :=a+\frac{\gamma_{1}}{(n+1)} a^{2}+\frac{n \gamma_{1}^{2}}{(n+1)^{2}} a^{3}, \\
b_{1} & :=b-\frac{\gamma_{1}}{(n+1)} a^{n-3} .
\end{aligned}
$$

Então um calculo direto nos mostra que $a_{1} b_{1}=0$, e uma vez que é claro que $a_{1}$ tem nilíndice máximo e que $b_{1}$ não pertence a $A_{a_{1}}$, segue que $\left(a_{1}, b_{1}\right)$ pertence a $\mathcal{P}(\mathcal{B})$. 
Utilizando (4.22) para calcular as potências de $a_{1}$, e calculando os produtos $b_{1}^{2}$ e $a_{1}^{2} b_{1}$ em termos das potências de $a_{1}$ obtemos que

$$
b_{1}^{2}=\alpha_{n-2}^{\prime} a_{1}^{n-2}+\alpha_{n-1}^{\prime} a_{1}^{n-1} \text { e } a_{1}^{2} b_{1}=a_{1}^{n-2} .
$$

Isto prova o lema.

Lema 4.50 Seja $\alpha_{2}$ não nulo e $\alpha_{1}$ em F. Então

$$
\mathcal{B}\left(\alpha_{2}, \alpha_{1}, 0,1,0\right) \cong \mathcal{B}\left(\alpha_{2}, 0,0,1,0\right)
$$

Prova: Seja $\mathcal{B}$ a álgebra $\mathcal{B}\left(\alpha_{2}, \alpha_{1}, 0,1,0\right)$ em relação a base determinada pelo par $(a, b)$. Definindo os seguintes elementos em $\mathcal{B}$,

$$
a_{1}:=-\frac{(n+1) \alpha_{1}}{(n-2) \alpha_{2}^{2}} b+a+\frac{\alpha_{1}}{(n-2) \alpha_{2}} a^{2}+\frac{\left[n \alpha_{1}^{2}-(n+1)(n-2) \alpha_{1}^{2}\right]}{(n-2)^{2} \alpha_{2}^{2}} a^{3}
$$

e

$$
b_{1}:=b+\frac{n \alpha_{1}}{(n-2) \alpha_{2}} a^{n-3},
$$

temos que $\left(a_{1}, b_{1}\right)$ está em $\mathcal{P}(\mathcal{B})$ e calculando os produtos $b_{1}^{2}$ e $a_{1}^{2} b_{1}$, obtemos as constantes de estrutura de $\mathcal{B}$ em relação a base determinada pelo par $\left(a_{1}, b_{1}\right)$, o que nos mostra o isomorfismo.

Observamos que se $\alpha_{2}=0$ então temos a álgebra $\mathcal{B}\left(0, \alpha_{1}, 0,1,0\right)$.

Se $\gamma_{2}=0$, temos a álgebra $\mathcal{B}\left(\alpha_{2}, \alpha_{1}, 0,0, \gamma_{1}\right)$. Neste caso, se $\gamma_{1}$ é não nulo, então fazendo $\beta=\frac{1}{\gamma_{1}}$ e $\lambda=1$ em (4.23), obtemos que

$$
\mathcal{B}\left(\alpha_{2}, \alpha_{1}, 0,0, \gamma_{1}\right) \cong \mathcal{B}\left(\alpha_{2}^{\prime}, \alpha_{1}^{\prime}, 0,0,1\right) .
$$

Lema 4.51 Seja $\alpha_{2}$ não nulo e $\alpha_{1}$ em F. Então

$$
\mathcal{B}\left(\alpha_{2}, \alpha_{1}, 0,0,1\right) \cong \mathcal{B}\left(\alpha_{2}, 0,0,0,1\right)
$$


Prova: Seja $\mathcal{B}$ a álgebra $\mathcal{B}\left(\alpha_{2}, \alpha_{1}, 0,0,1\right)$ em relação a base determinada pelo par $(a, b)$. Definimos os seguinte elementos em $\mathcal{B}$,

$$
a_{1}:=a+\frac{\alpha_{1}}{(n-2) \alpha_{2}} a^{2} \text { e } b_{1}:=b-\frac{\alpha_{1}}{(n-2) \alpha_{2}} a^{n-2} .
$$

Igual que no lema anterior obtemos que $\left(a_{1}, b_{1}\right)$ está em $\mathcal{P}(\mathcal{B})$ e

$$
b_{1}^{2}=\alpha_{2}^{\prime} a_{1}^{n-2} \text { e } a_{1}^{2} b_{1}=a_{1}^{n-3}
$$

o que demonstra o lema.

Se $\alpha_{2}=0$ então temos a álgebra $\mathcal{B}\left(0, \alpha_{1}, 0,0,1\right)$. Quando $\gamma_{1}=0$, temos a álgebra $\mathcal{B}\left(\alpha_{2}, \alpha_{1}, 0,0,0\right)$.

Lema 4.52 Seja $\alpha_{2}$ não nulo e $\alpha_{1}$ em F. Então

$$
\mathcal{B}\left(\alpha_{2}, \alpha_{1}, 0,0,0\right) \cong \mathcal{B}\left(\alpha_{2}, 0,0,0,0\right)
$$

Prova: Seja $\mathcal{B}$ a álgebra $\mathcal{B}\left(\alpha_{2}, \alpha_{1}, 0,0,0\right)$ em relação a base determinada pelo par $(a, b)$. Basta definir o seguinte elemento em $\mathcal{B}$,

$$
a_{1}:=a+\frac{\alpha_{1}}{(n-2) \alpha_{2}} a^{2}
$$

para obter que $\left(a_{1}, b\right)$ está em $\mathcal{P}(\mathcal{B})$ e

$$
b^{2}=\alpha_{2}^{\prime} a_{1}^{n-2} \text { e } a_{1}^{2} b=0,
$$

o que demonstra o lema.

Para $\alpha_{2} \neq 0$, temos a álgebra $\mathcal{B}\left(0, \alpha_{1}, 0,0,0\right)$.

De todo o anterior temos que se $\mathcal{B}$ é uma nilálgebra comutativa de p.a. de dimensão e nilíndice $n$, então existe uma base da forma $\left\{b, a, a^{2}, \ldots, a^{n-1}\right\}$, cujos produtos são dados por um da seguinte lista: 


$$
\begin{aligned}
& \mathcal{B}_{1}(\alpha, \theta): \quad b^{2}=\alpha a^{n-2}+\theta a^{n-1}, \quad a^{2} b=a^{n-3}, \quad a^{3} b=-a^{n-2}, \quad a^{4} b=a^{n-1} ; \\
& \mathcal{B}_{2}(\alpha): \quad b^{2}=\alpha a^{n-2}, \quad a^{2} b=a^{n-2}, \quad a^{3} b=-a^{n-1}, \quad \alpha \in F^{*} ; \\
& \mathcal{B}_{3}(\alpha): \quad b^{2}=\alpha a^{n-1}, \quad a^{2} b=a^{n-2}, \quad a^{3} b=-a^{n-1} ; \\
& \mathcal{B}_{4}(\alpha): \quad b^{2}=\alpha a^{n-2}, \quad a^{2} b=a^{n-1}, \quad \alpha \in F^{*} ; \\
& \mathcal{B}_{5}(\alpha): \quad b^{2}=\alpha a^{n-1}, \quad a^{2} b=a^{n-1} \\
& \mathcal{B}_{6}(\alpha): \quad b^{2}=\alpha a^{n-2}, \quad \alpha \in F^{*} ; \\
& \mathcal{B}_{7}(\alpha): \quad b^{2}=\alpha a^{n-1}, \quad \alpha \in F^{*} ;
\end{aligned}
$$

$\mathcal{B}_{8}:$

e os outros produtos não triviais são zero. Portanto toda álgebra desta classe é isomorfa a uma da lista anterior. Analisemos as condições de isomorfismo da lista anterior.

É claro que $\mathcal{B}_{8}$ não é isomorfa com as outras $\mathcal{B}_{i}$ por causa da dimensão do seu anulador. Por outro lado temos que se $\overline{\mathcal{B}}_{i}=\mathcal{B} / \operatorname{Anul}\left(\mathcal{B}_{i}\right)$, então a dimensão do anulador de $\overline{\mathcal{B}}_{5}$ e $\overline{\mathcal{B}}_{7}$ é 2 em quanto que a dimensão do anulador de $\overline{\mathcal{B}}_{i}$ é 1 para $i \neq 5,7$. Olhando agora para a dimensão do anulador de $\operatorname{Anul}\left(\overline{\mathcal{B}}_{i}\right)$, temos que é 1 para $i=1$, e é 2 para $i=2,3,4,6$. Portanto temos que

$$
\mathcal{B}_{1} \nsucceq \mathcal{B} i \text { para } i \neq 1 \text {. }
$$

As álgebras restantes da lista anterior, ficam divididas em dois conjuntos de álgebras não isomorfas, eles são:

$$
C 1: \mathcal{B}_{2}, \mathcal{B}_{3}, \mathcal{B}_{4}, \mathcal{B}_{6} . \quad C 2: \mathcal{B}_{5}, \mathcal{B}_{7}
$$

Lema 4.53 Para todo $\alpha$ não nulo,

$$
\mathcal{B}_{6}(\alpha) \cong \mathcal{B}_{4}(\alpha) .
$$

Prova: Seja $\alpha$ não nulo e $(a, b) \in \mathcal{P}\left(\mathcal{B}_{6}(\alpha)\right)$ tal que $b^{2}=\alpha a^{n-2}$ e os outros produtos não triviais são zero. Definindo os elementos

$$
a_{1}:=-\frac{1}{\alpha} b+a \text { e } b_{1}:=b+a^{n-3},
$$


obtemos que $\left(a_{1}, b_{1}\right) \in \mathcal{P}\left(\mathcal{B}_{6}(\alpha)\right)$ e que

$$
b_{1}^{2}=\alpha a_{1}^{n-2} \text { e } a_{1}^{2} b_{1}=a_{1}^{n-1}
$$

o que nos mostra o isomorfismo.

Antes de continuar com o análise das condições de isomorfismo daremos algumas propriedades para os pares $\left(a_{1}, b_{1}\right)$ em $\mathcal{P}(\mathcal{B})$, sendo $\mathcal{B}$ a álgebra $\mathcal{B}\left(\alpha_{2}, \alpha_{1}, \gamma_{3}, \gamma_{2}, \gamma_{1}\right)$. No seguinte lema nos referiremos a esta álgebra por $\mathcal{B}$.

Lema 4.54 Seja n maior ou igual que 9 e $(a, b)$ em $\mathcal{P}(\mathcal{B})$. Então para cada $\operatorname{par}\left(a_{1}, b_{1}\right)$ em $\mathcal{P}(\mathcal{B})$

$$
b_{1}=\beta b+\beta_{n-4} a^{n-4}+\beta_{n-3} a^{n-3}+\beta_{n-2} a^{n-2}+\beta_{n-1} a^{n-1},
$$

com $\beta$ não nulo e $\beta_{i}$ em $F$.

Prova: Seja $(a, b)$ em $\mathcal{P}(\mathcal{B})$ tal que $b^{2}=\alpha_{2} a^{n-2}+\alpha_{1} a^{n-1}, a^{2} b=\gamma_{3} a^{n-3}+$ $\gamma_{2} a^{n-2}+\gamma_{1} a^{n-1}, a^{3} b=-\gamma_{3} a^{n-2}-\gamma_{2} a^{n-1}, a^{4} b=\gamma_{3} a^{n-1}$ e os outros produtos não triviais são zero. Seja $\left(a_{1}, b_{1}\right)$ em $\mathcal{P}(\mathcal{B})$. O par $\left(a_{1}, b_{1}\right)$ exprime-se na base anterior como $a_{1}=\lambda b+\sum_{i=1}^{n-1} \lambda_{i} a^{i}$ e $b_{1}=\beta b+\sum_{i=1}^{n-1} \beta_{i} a^{i}$. Mostraremos por indução sobre $n$ que $b_{1} \in\langle b\rangle+\mathcal{B}^{n-4}$. Lembremos que por $(4.22), a_{1}^{n-1}=$ $\lambda_{1}^{n-1} \neq \mathrm{e} \operatorname{assim} \lambda_{1} \neq 0$.

Para $n=9$, temos que $b^{2}=\alpha_{7} a^{7}+\alpha_{8} a^{8}, a^{2} b=\gamma_{6} a^{6}+\gamma_{7} a^{7}+\gamma_{8} a^{8}, a^{3} b=$ $-\gamma_{6} a^{7}-\gamma_{7} a^{8}$ e $a^{4} b=\gamma_{6} a^{8}$. Logo

$$
\begin{aligned}
a_{1} b_{1}= & \lambda_{1} \beta_{1} a^{2}+\left(\lambda_{1} \beta_{2}+\lambda_{2} \beta_{1}\right) a^{3}+\left(\lambda_{1} \beta_{3}+\lambda_{2} \beta_{2}+\lambda_{3} \beta_{1}\right) a^{4}+\left(\lambda_{1} \beta_{4}+\lambda_{2} \beta_{3}\right. \\
& \left.+\lambda_{3} \beta_{2}+\lambda_{4} \beta_{1}\right) a^{5}+\left(\lambda_{1} \beta_{2} \gamma_{6}+\lambda_{1} \beta_{5}+\lambda_{1} \beta \gamma_{6}+\lambda_{2} \beta_{4} \lambda_{3} \beta_{3}+\lambda_{4} \beta_{2}+\right. \\
& \left.\lambda_{5} \beta_{1}\right) a^{6}+\left(\lambda \beta_{2} \alpha_{7}+\lambda \beta_{2} \gamma_{7}-\lambda \beta_{3} \gamma_{6}+\lambda_{1} \beta_{6}+\lambda_{2} \beta \gamma_{7}+\lambda_{2} \beta_{5}-\lambda_{3} \beta \gamma_{6}\right. \\
& \left.+\lambda_{3} \beta_{4}+\lambda_{4} \beta_{3}+\lambda_{5} \beta_{2}+\lambda_{6} \beta_{1}\right) a^{7}+\left(\lambda \beta \alpha_{8}+\lambda \beta_{2} \gamma_{8}-\lambda \beta_{3} \gamma_{7}+\lambda \beta_{4} \gamma_{6}\right. \\
& +\lambda_{1} \beta_{7}+\lambda_{2} \beta \gamma_{8}+\lambda_{2} \beta_{6}-\lambda_{3} \beta \gamma_{7}+\lambda_{3} \beta_{5}+\lambda_{4} \beta \gamma_{6}+\lambda_{4} \beta_{4}+\lambda_{5} \beta_{3}+ \\
& \left.\lambda_{6} \beta_{2}+\lambda_{7} \beta_{1}\right) a^{8} \\
= & 0,
\end{aligned}
$$


e uma vez que $\lambda_{1} \neq 0$, segue que $\beta_{1}=0$. Igualmente concluímos que $\beta_{2}=\beta_{3}=\beta_{4}=0$, portanto

$$
b_{1}=\beta b+\beta_{5} a^{5}+\beta_{6} a^{6}+\beta_{7} a^{7}+\beta_{8} a^{8}, \quad \text { com } \beta \neq 0 .
$$

Assim

$$
b_{1} \in\langle b\rangle+\mathcal{B}^{5} \text { e } \beta \neq 0 .
$$

Suponhamos, por hipótese de indução que $b_{1} \in\langle b\rangle+\mathcal{B}^{k-4}$ e $\beta \neq 0$, para $9 \leq k \leq n-1$, e demonstremos para $k=n$.

Sejam $(a, b),\left(a_{1}, b_{1}\right) \in \mathcal{P}(\mathcal{B})$ e $\mathcal{B}=\left\langle b, a, \ldots, a^{n-1}\right\rangle$. Como $\left\langle a^{n-1}\right\rangle$ é um ideal de $\mathcal{B}$, temos que $\overline{\mathcal{B}}=\mathcal{B} /\left\langle a^{n-1}\right\rangle=\left\langle\bar{b}, \bar{a}, \ldots, \bar{a}^{n-1}\right\rangle$, e é claro que $(\bar{a}, \bar{b}),\left(\bar{a}_{1}, \bar{b}_{1}\right) \in \mathcal{P}(\overline{\mathcal{B}})$. Pela hipótese de indução, $\bar{b}_{1} \in\langle\bar{b}\rangle+\overline{\mathcal{B}}^{n-5}$ e $\beta \neq 0$, logo, sem perda de generalidade, podemos utilizar os mesmos escalares, e dizer que

$$
b_{1}=\beta b+\beta_{n-5} a^{5}+\beta_{n-4} a^{n-4}+\beta_{n-3} a^{n-3}+\beta_{n-2} a^{n-2}+\beta_{n-1} a^{n-1} .
$$

Logo

$$
\begin{aligned}
a_{1} b_{1}= & \lambda_{1} \beta_{n-5} a^{n-4}+\left(\lambda_{2} \beta \gamma_{n-3}+\lambda_{1} \beta_{n-4}+\lambda_{2} \beta_{n-5}\right) a^{n-3}+\left(\lambda \beta \alpha_{n-2}+\right. \\
& \left.\lambda_{2} \beta \gamma_{n-2}-\lambda_{3} \beta \gamma_{n-3}+\lambda_{1} \beta_{n-3}+\lambda_{2} \beta_{n-4}+\lambda_{3} \beta_{n-5}\right) a^{n-2} \\
& +\left(\lambda \beta \alpha_{n-1}+\lambda_{2} \beta \gamma_{n-1}-\lambda_{3} \beta \gamma_{n-2}+\lambda_{4} \beta \gamma_{n-3}+\lambda_{1} \beta_{n-2}+\lambda_{2} \beta_{n-3}\right. \\
& \left.+\lambda_{3} \beta_{n-4}+\lambda_{4} \beta_{n-5}\right) a^{n-1} \\
= & 0
\end{aligned}
$$

e como $\lambda_{1} \neq 0$, segue que $\beta_{n-5}=0$, portanto

$$
\begin{aligned}
a_{1} b_{1}= & \left(\lambda_{2} \beta \gamma_{n-3}+\lambda_{1} \beta_{n-4}\right) a^{n-3}+\left(\lambda \beta \alpha_{n-2}+\lambda_{2} \beta \gamma_{n-2}-\lambda_{3} \beta \gamma_{n-3}+\lambda_{1} \beta_{n-3}\right. \\
& \left.+\lambda_{2} \beta_{n-4}\right) a^{n-2}+\left(\lambda \beta \alpha_{n-1}+\lambda_{2} \beta \gamma_{n-1}-\lambda_{3} \beta \gamma_{n-2}+\lambda_{4} \beta \gamma_{n-3}+\lambda_{1} \beta_{n-2}\right. \\
& \left.+\lambda_{2} \beta_{n-3}+\lambda_{3} \beta_{n-4}\right) a^{n-1} \\
= & 0
\end{aligned}
$$

e assim

$$
b_{1}=\beta b+\beta_{n-4} a^{n-4}+\beta_{n-3} a^{n-3}+\beta_{n-2} a^{n-2}+\beta_{n-1} a^{n-1} \text { com } \beta \neq 0,
$$


isto é

$$
b_{1} \in\langle b\rangle+\mathcal{B}^{n-4} \text { para } n \geq 9 .
$$

Do lema anterior é claro que

$$
b_{1}^{2}=\beta^{2} b^{2}, \quad \operatorname{com} \beta \neq 0,
$$

e assim para $\left(a_{1}, b_{1}\right)$ em $\mathcal{P}\left(\mathcal{B}_{i}\right)$, com $i=2,6$, temos que

$$
b_{1}^{2}=\frac{\beta^{2}}{\lambda_{1}^{n-2}} \alpha a_{1}^{n-2}+\frac{\beta^{2} \lambda_{2}(n-2)}{\lambda_{1}} \alpha a_{1}^{n-1} \operatorname{com} \beta, \lambda_{1}, \alpha \neq 0,
$$

e para $\left(a_{1}, b_{1}\right)$ em $\mathcal{P}\left(\mathcal{B}_{j}\right)$, com $j=3,5,7$, temos que

$$
b_{1}^{2}=\frac{\beta^{2}}{\lambda_{1}^{n-1}} \alpha a_{1}^{n-1} .
$$

Portanto para termos o isomorfismo entre uma álgebra $\mathcal{B}_{i}$ e uma álgebra $\mathcal{B}_{j}$, é preciso que $\frac{\beta^{2}}{\lambda_{1}^{n-2}} \alpha=0$, o que não é possível. Uma vez que vimos que $\mathcal{B}_{3}$ não é isomorfa com $\mathcal{B}_{5}$ e $\mathcal{B}_{7}$, temos que

$$
\mathcal{B}_{3} \nsucceq \mathcal{B}_{i} \text { para } i \neq 3 \text {. }
$$

Assim restam por analisar no conjunto $C 1$ as álgebras $\mathcal{B}_{2}$ e $\mathcal{B}_{6}$, e no conjunto $C 2$, as álgebras $\mathcal{B}_{5}$ e $\mathcal{B}_{7}$. Antes disso citamos as seguintes igualdades que serão utilizadas nos seguintes lemas, elas decorrem da equação (4.22) que caracteriza as potências de um elemento $x$ neste tipo de álgebras. De aqui por diante $a_{1}=\lambda b+\sum_{i=1}^{n-1} \lambda_{i} a^{i}$.

$$
\begin{aligned}
a_{1}^{2} & =\lambda_{1}^{2} a^{2}+2 \lambda_{1} \lambda_{2} a^{3}+\left[\lambda_{2}^{2}+2 \lambda_{1} \lambda_{3}\right] a^{4}+d, \\
a_{1}^{n-3} & =\lambda_{1}^{n-3} a^{n-3}+(n-3) \lambda_{1}^{n-4} \lambda_{2} a^{n-2}+(n-3) \lambda_{1}^{n-4}\left[\lambda_{3}+\frac{(n-4)}{2 \lambda_{1}} \lambda_{2}^{2}\right] a^{n-1}, \\
a_{1}^{n-2} & =\lambda_{1}^{n-2} a^{n-2}+(n-2) \lambda_{1}^{n-3} \lambda_{2} a^{n-1}, \\
a_{1}^{n-1} & =\lambda_{1}^{n-1} a^{n-1}, \\
\operatorname{com} d & \in \mathcal{B}^{5} \text { e } \lambda_{1} \neq 0 .
\end{aligned}
$$


Lema 4.55 Seja $\mathcal{B}$ a álgebra $\mathcal{B}_{7}(\alpha)$. Então para cada par $\left(a_{1}, b_{1}\right)$ em $\mathcal{P}(\mathcal{B})$,

$$
b_{1}^{2}=\frac{\beta^{2}}{\lambda_{1}^{n-1}} \alpha a_{1}^{n-1} \quad \text { e } a_{1}^{2} b_{1}=0, \quad \text { com } \beta, \lambda_{1} \neq 0 .
$$

Prova: Seja $\alpha$ não nulo e $(a, b)$ em $\mathcal{P}(B)$ tal que $b^{2}=\alpha a^{n-1}, a^{2} b=0$ e os outros produtos não triviais são zero. Se $\left(a_{1}, b_{1}\right)$ está em $\mathcal{P}(B)$, então por (4.24) temos que

$$
\begin{aligned}
a_{1} b_{1}= & \lambda_{1} \beta_{n-4} a^{n-3}+\left(\lambda_{1} \beta_{n-3}+\lambda_{2} \beta_{n-4}\right) a^{n-2}+\left(\lambda \beta \alpha+\lambda_{1} \beta_{n-2}+\right. \\
& \left.\lambda_{2} \beta_{n-3}+\lambda_{3} \beta_{n-4}\right) a^{n-1}=0,
\end{aligned}
$$

e como $\lambda_{1} \neq 0$, segue que $\beta_{n-4}=\beta_{n-3}=0$, assim,

$$
b_{1}=\beta b+\beta_{n-2} a^{n-2}+\beta_{n-1} a^{n-1} \text { com } \beta \neq 0 .
$$

Utilizando (4.25), calculamos os produtos $b_{1}^{2}, a_{1}^{2} b_{1}$ e obtemos que $b_{1}^{2}=$ $\beta^{2} \alpha a_{1}^{n-1}$ e $a_{1}^{2} b_{1}=0$, que em termos das potências de $a_{1}$ são

$$
b_{1}^{2}=\frac{\beta^{2}}{\lambda_{1}^{n-1}} \alpha a_{1}^{n-1} \text { e } a_{1}^{2} b_{1}=0,
$$

o que demonstra o lema.

Do lema anterior é claro que $\mathcal{B}_{7} \nsucceq \mathcal{B}_{5}$. Portanto

$$
\mathcal{B}_{5} \not \mathcal{B}_{i} \text { e } \mathcal{B}_{7} \not \mathcal{B}_{j}, \quad \text { para } i \neq 5 \text { e } j \neq 7 \text {. }
$$

Temos também, como uma consequência imediata do lema, o seguinte corolário.

Corolário 4.56 Seja a não nulo e $\alpha^{\prime}$ em F. Então

$$
\mathcal{B}_{7}(\alpha) \cong \mathcal{B}_{7}\left(\alpha^{\prime}\right)
$$

se e somente se, existirem $\lambda$ e $\beta$ em $F^{*}$, tais que $\alpha^{\prime}=\frac{\beta^{2}}{\lambda^{n-1}} \alpha$. 
Resta então analisar as álgebras do conjunto $C 1$. Faremos isto do mesmo jeito que fizemos para $C 2$, portanto omitiremos alguns detalhes na demonstração do seguinte lema.

Lema 4.57 Seja $\mathcal{B}$ a álgebra $\mathcal{B}_{6}(\alpha)$. Então para cada par $\left(a_{1}, b_{1}\right)$ em $\mathcal{P}(\mathcal{B})$,

$$
b_{1}^{2}=\frac{\beta^{2}}{\lambda_{1}^{n-2}} \alpha a_{1}^{n-2}-\frac{\beta^{2} \lambda_{2}(n-2)}{\lambda_{1}^{n}} \alpha a_{1}^{n-1} \quad \text { e } a_{1}^{2} b_{1}=\frac{\beta_{n-3}}{\lambda_{1}^{n-3}} a_{1}^{n-1} .
$$

Prova: Seja $\alpha$ não nulo e $(a, b)$ em $\mathcal{P}(B)$ tal que $b^{2}=\alpha a^{n-2}, a^{2} b=0$ e os outros produtos não triviais são zero. Seja $\left(a_{1}, b_{1}\right)$ em $\mathcal{P}(B)$. Então por (4.24) temos que $\beta_{n-4}=0$ e assim,

$$
b_{1}=\beta b+\beta_{n-3} a^{n-3}+\beta_{n-2} a^{n-2}+\beta_{n-1} a^{n-1} \text { com } \beta \neq 0 .
$$

Logo

$$
b_{1}^{2}=\beta^{2} \alpha a^{n-2}=\frac{\beta^{2}}{\lambda_{1}^{n-2}} \alpha a_{1}^{n-2}-\frac{\beta^{2} \lambda_{2}(n-2)}{\lambda_{1}^{n}} \alpha a_{1}^{n-1}
$$

$\mathrm{e}$

$$
a_{1}^{2} b_{1}=\lambda_{1}^{2} \beta_{n-3} a^{n-1}=\frac{\beta_{n-3}}{\lambda_{1}^{n-3}} a_{1}^{n-1} .
$$

Do lema é claro que $\mathcal{B}_{6}(\alpha) \varsubsetneqq \mathcal{B}_{2}(\alpha)$. Portanto

$$
\mathcal{B}_{6} ¥ \mathcal{B}_{i} \text { e } \mathcal{B}_{2} \not \mathcal{B}_{j} \text { para } i \neq 6 \text { e } j \neq 2 \text {. }
$$

Como uma consequência do lema anterior temos o seguinte corolário.

Corolário 4.58 Seja $\alpha$ não nulo e $\alpha^{\prime}$ em F. Então

$$
\mathcal{B}_{6}(\alpha) \cong \mathcal{B}_{6}\left(\alpha^{\prime}\right)
$$

se, e somente se, existirem $\lambda$ e $\beta$ em $F^{*}$, tais que $\alpha^{\prime}=\frac{\beta^{2}}{\lambda^{n-2}} \alpha$.

Podemos concluir de todo o anterior (excluindo a álgebra $\mathcal{B}_{4}$ ), que

$$
\mathcal{B}_{i} \not \mathcal{B}_{j} \text { para } i \neq j \text {. }
$$

Estudemos agora as classes dadas por $\mathcal{B}_{1}, \mathcal{B}_{2}, \mathcal{B}_{3}$ e $\mathcal{B}_{5}$. 
Lema 4.59 Seja $\mathcal{B}$ a álgebra $\mathcal{B}_{5}(\alpha)$. Então para cada par $\left(a_{1}, b_{1}\right)$ em $\mathcal{P}(\mathcal{B})$,

$$
b_{1}^{2}=\frac{\beta^{2}}{\lambda_{1}^{n-1}} \alpha a_{1}^{n-1} \quad \text { e } a_{1}^{2} b_{1}=\frac{\beta}{\lambda_{1}^{n-3}} a_{1}^{n-1} .
$$

Prova: Seja $\alpha$ em $F$ e $(a, b)$ em $\mathcal{P}(B)$ tal que $b^{2}=\alpha a^{n-1}, a^{2} b=a^{n-1}$ e os outros produtos não triviais são zero. Seja $\left(a_{1}, b_{1}\right)$ em $\mathcal{P}(B)$, então por (4.24) temos que $\beta_{n-4}=\beta_{n-3}=0, \operatorname{logo}$

$$
b_{1}=\beta b+\beta_{n-2} a^{n-2}+\beta_{n-1} a^{n-1} \quad \text { com } \beta \neq 0,
$$

e assim

$$
b_{1}^{2}=\beta^{2} \alpha a^{n-1}=\frac{\beta^{2}}{\lambda_{1}^{n-1}} \alpha a_{1}^{n-1} \text { e } a_{1}^{2} b_{1}=\lambda_{1}^{2} \beta a^{n-1}=\frac{\beta}{\lambda_{1}^{n-3}} a_{1}^{n-1} .
$$

Notemos que para $\alpha^{\prime}$ em $F$, a álgebra $\mathcal{B}_{5}\left(\alpha^{\prime}\right)$ é isomorfa a álgebra $\mathcal{B}_{5}(\alpha)$ se, e somente se, $\beta=\lambda_{1}^{n-3}$ e $\alpha^{\prime}=\frac{\beta^{2}}{\lambda_{1}^{n-1}} \alpha=\lambda_{1}^{n-5} \alpha$.

Corolário 4.60 Sejam $\alpha$ e $\alpha^{\prime}$ em F. Então

$$
\mathcal{B}_{5}(\alpha) \cong \mathcal{B}_{5}\left(\alpha^{\prime}\right)
$$

se, e somente se, existir $\lambda$ em $F^{*}$, tal que $\alpha^{\prime}=\lambda^{n-5} \alpha$.

Lema 4.61 Seja $\mathcal{B}$ a álgebra $\mathcal{B}_{3}(\alpha)$. Então para cada par $\left(a_{1}, b_{1}\right)$ em $\mathcal{P}(\mathcal{B})$,

$$
b_{1}^{2}=\frac{\beta^{2}}{\lambda_{1}^{n-1}} \alpha a_{1}^{n-1} \quad \text { e } a_{1}^{2} b_{1}=\frac{\beta}{\lambda_{1}^{n-4}} a^{n-2}+\frac{\lambda_{1} \beta_{n-3}-n \lambda_{2} \beta}{\lambda_{1}^{n-2}} a_{1}^{n-1} .
$$

Prova: Seja $\alpha$ em $F$ e $(a, b)$ em $\mathcal{P}(B)$ tal que $b^{2}=\alpha a^{n-1}, a^{2} b=a^{n-2}, a^{3} b=$ $-a^{n-1}$ e os outros produtos não triviais são zero. Seja $\left(a_{1}, b_{1}\right)$ em $\mathcal{P}(B)$, então por (4.24) temos que $\beta_{n-4}=0$ e assim,

$$
b_{1}=\beta b+\beta_{n-3} a^{n-3}+\beta_{n-2} a^{n-2}+\beta_{n-1} a^{n-1} \quad \text { com } \beta \neq 0,
$$


portanto

$$
b_{1}^{2}=\beta^{2} \alpha a^{n-1}=\frac{\beta^{2}}{\lambda_{1}^{n-1}} \alpha a_{1}^{n-1}
$$

$\mathrm{e}$

$a_{1}^{2} b_{1}=\lambda_{1}^{2} \beta a^{n-2}+\left(\lambda_{1}^{2} \beta_{n-3}-2 \lambda_{1} \lambda_{2} \beta\right) a^{n-1}=\frac{\beta}{\lambda_{1}^{n-4}} a^{n-2}+\frac{\lambda_{1} \beta_{n-3}-n \lambda_{2} \beta}{\lambda_{1}^{n-2}} a_{1}^{n-1}$.

Temos do lema anterior, que para $\alpha^{\prime}$ em $F$, a álgebra $\mathcal{B}_{3}\left(\alpha^{\prime}\right)$ é isomorfa a álgebra $\mathcal{B}_{3}(\alpha)$ se, e somente se, $\beta_{n-3}=\frac{n \lambda_{2} \beta}{\lambda_{1}}, \beta=\lambda_{1}^{n-4}$ e $\alpha^{\prime}=\frac{\beta^{2}}{\lambda_{1}^{n-1}} \alpha=$ $\lambda_{1}^{n-7} \alpha$.

Corolário 4.62 Para $\alpha$ e $\alpha^{\prime}$ em $F$

$$
\mathcal{B}_{3}(\alpha) \cong \mathcal{B}_{3}\left(\alpha^{\prime}\right)
$$

se, e somente se, existir $\lambda$ não nulo tal que $\alpha^{\prime}=\lambda^{n-7} \alpha$.

Lema 4.63 Seja $\alpha$ não nulo e $\mathcal{B}$ a álgebra $\mathcal{B}_{2}(\alpha)$. Então para cada par $\left(a_{1}, b_{1}\right)$ em $\mathcal{P}(\mathcal{B})$,

$$
b_{1}^{2}=\frac{\beta^{2}}{\lambda_{1}^{n-2}} \alpha a_{1}^{n-2}-\frac{\beta^{2}(n-2) \lambda_{2}}{\lambda_{1}^{n}} \alpha a_{1}^{n-1}
$$

$e$

$$
a_{1}^{2} b_{1}=\frac{\beta}{\lambda_{1}^{n-4}} a_{1}^{n-2}-\frac{\beta \lambda_{2} n-\beta_{n-3} \lambda_{1}}{\lambda_{1}^{n-2}} a_{1}^{n-1} .
$$

Prova: Seja $\alpha$ não nulo e $(a, b)$ em $\mathcal{P}(B)$ tal que $b^{2}=\alpha a^{n-2}, a^{2} b=a^{n-2}, a^{3} b=$ $-a^{n-1}$ e os outros produtos não triviais são zero. Seja $\left(a_{1}, b_{1}\right)$ em $\mathcal{P}(B)$, então por (4.24) temos que $\beta_{n-4}=0$ e assim,

$$
b_{1}=\beta b+\beta_{n-3} a^{n-3}+\beta_{n-2} a^{n-2}+\beta_{n-1} a^{n-1} \quad \text { com } \beta \neq 0,
$$

portanto

$$
b_{1}^{2}=\beta^{2} \alpha a^{n-2}=\frac{\beta^{2}}{\lambda_{1}^{n-2}} \alpha a_{1}^{n-2}-\frac{\beta^{2}(n-2) \lambda_{2}}{\lambda_{1}^{n}} \alpha a_{1}^{n-1}
$$


e

$$
\begin{aligned}
a_{1}^{2} b_{1} & =\lambda_{1}^{2} \beta a^{n-2}+\left(\lambda_{1}^{2} \beta_{n-3}-2 \lambda_{1} \lambda_{2} \beta\right) a^{n-1} \\
& =\frac{\beta}{\lambda_{1}^{n-4}} a_{1}^{n-2}-\frac{\beta \lambda_{2} n-\beta_{n-3} \lambda_{1}}{\lambda_{1}^{n-2}} a_{1}^{n-1}
\end{aligned}
$$

Assim, temos do lema anterior que para $\alpha^{\prime}$ em $F^{*}, \mathcal{B}_{2}\left(\alpha^{\prime}\right) \cong \mathcal{B}_{2}(\alpha)$ se, e somente se, $\lambda_{2}=\beta_{n-3}=0, \beta=\lambda_{1}^{n-4}$ e $\alpha^{\prime}=\frac{\beta^{2}}{\lambda_{1}^{n-2}} \alpha=\lambda_{1}^{n-6} \alpha$.

Corolário 4.64 Se $\alpha$ e $\alpha^{\prime}$ são não nulos, então

$$
\mathcal{B}_{2}(\alpha) \cong \mathcal{B}_{2}\left(\alpha^{\prime}\right)
$$

se, e somente se, existir $\lambda$ em $F^{*}$, tal que $\alpha^{\prime}=\lambda^{n-6} \alpha$.

Lema 4.65 Seja $\mathcal{B}$ a álgebra $\mathcal{B}_{1}(\alpha, \theta)$. Então para cada par $\left(a_{1}, b_{1}\right)$ em $\mathcal{P}(\mathcal{B})$,

$$
b_{1}^{2}=\frac{\beta^{2}}{\lambda_{1}^{n-2}} \alpha a_{1}^{n-2}+\frac{\beta^{2}\left(\theta-(n-2) \lambda_{2} \alpha\right)}{\lambda_{1}^{n}} a_{1}^{n-1}
$$

$e$

$$
\begin{aligned}
a_{1}^{2} b_{1}= & \frac{\beta}{\lambda_{1}^{n-5}} a_{1}^{n-3}+\frac{\left(\lambda_{1} \beta_{n-4}-(n-1) \lambda_{2} \beta\right)}{\lambda_{1}^{n-3}} a_{1}^{n-2}+ \\
& \frac{\left[-2(n-5) \lambda_{1} \lambda_{3} \beta-2(n-4) \lambda_{1} \lambda_{2} \beta_{n-4}+\lambda_{2}^{2} \beta(n+3)(n-2)+2 \lambda_{1}^{2} \beta_{n-3}\right]}{2 \lambda_{1}^{n-1}} a_{1}^{n-1} .
\end{aligned}
$$

Prova: Seja $\alpha$ em $F$ e $(a, b)$ em $\mathcal{P}(B)$ tal que $b^{2}=\alpha a^{n-2}+\theta a^{n-1}, a^{2} b=$ $a^{n-3}, a^{3} b=-a^{n-2}, a^{4} b=a^{n-1}$ e os outros produtos não triviais são zero. Seja $\left(a_{1}, b_{1}\right)$ em $\mathcal{P}(B)$, então por (4.24) temos que

$$
b_{1}=\beta b+\beta_{n-4} a^{n-4}+\beta_{n-3} a^{n-3}+\beta_{n-2} a^{n-2}+\beta_{n-1} a^{n-1} \quad \text { com } \beta \neq 0,
$$

portanto

$$
b_{1}^{2}=\beta^{2} \alpha a^{n-2}+\beta^{2} \theta a^{n-1}=\frac{\beta^{2}}{\lambda_{1}^{n-2}} \alpha a_{1}^{n-2}+\frac{\beta^{2}\left(\theta-(n-2) \lambda_{2} \alpha\right)}{\lambda_{1}^{n}} a_{1}^{n-1}
$$


e

$$
\begin{aligned}
& a_{1}^{2} b_{1}=\lambda_{1}^{2} \beta a^{n-3}+\lambda_{1}\left(\lambda_{1} \beta_{n-4}-2 \lambda_{2} \beta\right) a^{n-2}+\left(2 \lambda_{1} \lambda_{3} \beta+2 \lambda_{1} \lambda_{2} \beta_{n-4}+\lambda_{1}^{2} \beta_{n-3}\right. \\
& \left.+\lambda_{2}^{2} \beta\right) a^{n-1} \\
& =\frac{\beta}{\lambda_{1}^{n-5}} a_{1}^{n-3}+\frac{\left(\lambda_{1} \beta_{n-4}-(n-1) \lambda_{2} \beta\right)}{\lambda_{1}^{n-3}} a_{1}^{n-2}+ \\
& \frac{\left[-2(n-5) \lambda_{1} \lambda_{3} \beta-2(n-4) \lambda_{1} \lambda_{2} \beta_{n-4}+\lambda_{2}^{2} \beta(n+3)(n-2)+2 \lambda_{1}^{2} \beta_{n-3}\right]}{2 \lambda_{1}^{n-1}} a_{1}^{n-1} .
\end{aligned}
$$

Queremos ressaltar que no lema anterior tínhamos que

$$
b_{1}=\beta b+\beta_{n-4} a^{n-4}+\beta_{n-3} a^{n-3}+\beta_{n-2} a^{n-2}+\beta_{n-1} a^{n-1} \text { com } \beta \neq 0,
$$

e uma vez que $a_{1} b_{1}=0$, temos pela equação (4.24) que

$$
\begin{aligned}
\left(\lambda_{2} \beta+\right. & \left.\lambda_{1} \beta_{n-4}\right) a^{n-3}+\left(\lambda \beta \alpha-\lambda_{3} \beta+\lambda_{1} \beta_{n-3}+\lambda_{2} \beta_{n-4}\right) a^{n-2} \\
& +\left(\lambda \beta \theta+\lambda_{4} \beta+\lambda_{1} \beta_{n-2}+\lambda_{2} \beta_{n-3}+\lambda_{3} \beta_{n-4}\right) a^{n-1}=0
\end{aligned}
$$

portanto $\beta_{n-4}=-\frac{\lambda_{2} \beta}{\lambda_{1}}$ e $\lambda_{1} \beta_{n-4}-(n-1) \lambda_{2} \beta=-n \lambda_{2} \beta$. Assim utilizando esta ultima igualdade, temos que para um $\alpha^{\prime}$ em $F$, a álgebra $\mathcal{B}_{1}\left(\alpha^{\prime}, \theta^{\prime}\right)$ é isomorfa à álgebra $\mathcal{B}_{1}(\alpha, \theta)$ se, e somente se, $\beta=\lambda_{1}^{n-5}, \lambda_{2}=\beta_{n-4}=0, \beta_{n-3}=\frac{(n-5) \lambda_{3} \beta}{\lambda_{1}}$ e em consequência, $\alpha^{\prime}=\frac{\beta^{2}}{\lambda_{1}^{n-2}} \alpha=\lambda_{1}^{n-8} \alpha$ e $\theta^{\prime}=\frac{\beta^{2}}{\lambda_{1}^{n}} \theta=\lambda_{1}^{n-10} \theta$.

Corolário 4.66 Sejam $\alpha, \theta, \alpha^{\prime}$ e $\theta^{\prime}$ em F. Então

$$
\mathcal{B}_{1}(\alpha, \theta) \cong \mathcal{B}_{1}\left(\alpha^{\prime}, \theta^{\prime}\right)
$$

se, e somente se, existirem $\lambda, \lambda_{1}$ em $F^{*}$, tal que $\alpha^{\prime}=\lambda^{n-8} \alpha \quad$ e $\theta^{\prime}=\lambda_{1}^{n-10} \theta$.

Podemos finalmente enunciar os seguintes dois teoremas que dão uma classificação para dimensão e nilíndice $n$, quando $n$ é par e quando $n$ é ímpar.

Teorema 4.67 Seja n par e maior ou igual a 8. Uma nilálgebra comutativa de p.a. de dimensão e nilíndice n, é isomorfa a uma e somente uma álgebra 
com base $\left\{b, a, a^{2}, \ldots, a^{n-1}\right\}$, e produtos não triviais e não zero conforme um dos casos que aparecem na seguinte lista:

$$
\begin{aligned}
& \mathcal{A}_{1}(\alpha, \theta): \quad b^{2}=\alpha a^{n-2}+\theta a^{n-1}, \quad a^{2} b=a^{n-3}, \quad a^{3} b=-a^{n-2}, \quad a^{4} b=a^{n-1} ; \\
& \mathcal{A}_{2}(\alpha): \quad b^{2}=\alpha a^{n-2}, \quad a^{2} b=a^{n-2}, \quad a^{3} b=-a^{n-1}, \quad\left[\rho^{n-6}\right] ; \\
& \mathcal{A}_{3}(\alpha): \quad b^{2}=\alpha a^{n-1}, \quad a^{2} b=a^{n-2}, \quad a^{3} b=-a^{n-1}, \quad\left[\rho^{n-7}\right] ; \\
& \mathcal{A}_{4}(\alpha): \quad b^{2}=\alpha a^{n-1}, \quad a^{2} b=a^{n-1}, \quad\left[\rho^{n-5}\right] ; \\
& \mathcal{A}_{5}(\alpha): \quad b^{2}=\alpha a^{n-2}, \\
& \mathcal{A}_{6}(\alpha): \quad b^{2}=a^{n-1}
\end{aligned}
$$

e para todo $\alpha^{\prime}$ e $\theta^{\prime}$ em $F$, temos que $\mathcal{A}_{1}(\alpha, \theta) \cong \mathcal{A}_{1}\left(\alpha^{\prime}, \theta^{\prime}\right)$, se e somente se existirem $\rho, \lambda$ não nulos tais que $\alpha^{\prime}=\rho^{n-8} \alpha$ e $\theta^{\prime}=\lambda^{n-10} \theta$.

Teorema 4.68 Seja $n$ impar e maior ou igual a 9. Uma nilálgebra comutativa de p.a. de dimensão e nilíndice n, é isomorfa a uma e somente uma álgebra com base $\left\{b, a, a^{2}, \ldots, a^{n-1}\right\}$, e produtos não triviais e não zero conforme um dos casos que aparecem na seguinte lista:

$$
\begin{aligned}
& \mathcal{A}_{1}(\alpha, \theta): \quad b^{2}=\alpha a^{n-2}+\theta a^{n-1}, \quad a^{2} b=a^{n-3}, \quad a^{3} b=-a^{n-2}, \quad a^{4} b=a^{n-1} ; \\
& \mathcal{A}_{2}(\alpha): \quad b^{2}=\alpha a^{n-2}, \quad a^{2} b=a^{n-2}, \quad a^{3} b=-a^{n-1} ; \quad\left[\rho^{n-6}\right] ; \\
& \mathcal{A}_{3}(\alpha): \quad b^{2}=\alpha a^{n-1}, \quad a^{2} b=a^{n-2}, \quad a^{3} b=-a^{n-1}, \quad\left[\rho^{n-7}\right] ; \\
& \mathcal{A}_{4}(\alpha): \quad b^{2}=\alpha a^{n-1}, \quad a^{2} b=a^{n-1}, \quad\left[\rho^{n-5}\right] ; \\
& \mathcal{A}_{5}(\alpha): \quad b^{2}=a^{n-2} \\
& \mathcal{A}_{6}(\alpha): \quad b^{2}=\alpha a^{n-1}, \quad\left[\rho^{2}\right] ;
\end{aligned}
$$

e para todo $\alpha^{\prime}$ e $\theta^{\prime}$ em $F$, temos que $\mathcal{A}_{1}(\alpha, \theta) \cong \mathcal{A}_{1}\left(\alpha^{\prime}, \theta^{\prime}\right)$, se e somente se existirem $\rho, \lambda$ não nulos tais que $\alpha^{\prime}=\rho^{n-8} \alpha$ e $\theta^{\prime}=\lambda^{n-10} \theta$. 


\section{Referências Bibliográficas}

[1] Albert, A.A. On the Power-associativity of Rings. Summ. Bras. Math. 1948, 2 (2), 21-33.

[2] Albert, A.A. Power-associative Rings. Trans Am. Math. Soc. 1948, 64, 552-593.

[3] Correa, I. On the Index of Nilpotence of Jordan Nilalgebras. Int. J. Math. Game Theory Algebra. 2001, 11 (2), 73-79.

[4] Correa, I.; Hentzel, I.R. On Solvability of Noncommutative Powerassociative Nilalgebras. J. Algebra. 2001, 240, 98-102.

[5] Correa, I.; Hentzel, I.R.; Julca, P.P.; Peresi, L.A. Nilpotent Linear Transformations and the Solvability of Power-associative Nilalgebras. Linear Algebra Appl. 2005, 396, 35-53.

[6] Correa, I.; Hentzel, I.R.; Peresi, L.A. On the Solvability of the Commutative Power-associative Nilalgebras of Dimension 6. Linear Algebra Appl. 2003, 369, 185-192.

[7] Correa, I.; Peresi, L.A. On the Solvability of the Five Dimensional Commutative Power-associative Nilalgebras. Result. Math. 2001, 39, 23-27. 
[8] Correa, I.; Suazo, A. On a Class of Commutative Power-associative Nilalgebras. J. Algebra. 1999, 215, 412-417.

[9] Dedkov, A.I. Power-associative Algebras having a Nil-basis. Algebra and Logic. 1985, 24 (3), 163-170.

[10] Elgueta, L.; Suazo, A. Jordan Nilalgebras of Nilindex n and Dimension n+1. Comm. Algebra. 2002, 30, 5547-5561.

[11] Elgueta, L.; Suazo, A. Solvability of Commutative Power-associative Nilalgebras of Nilindex 4 and Dimension $\leq$ 8. Proyecciones. 2004, 23 (2), 123-129.

[12] Gerstenhaber, M.; Myung, H.C. On Commutative Power-associative Nilalgebras of Low Dimension. Proc. Amer. Math. Soc. 1975, 48, 29-32.

[13] Gerstenhaber, M. On Nilalgebras and Linear Varieties of Nilpotent Matrices. II, Duke Math. J. 1960, 27, 21-31.

[14] Gerstenhaber, M. On Nilalgebras and Linear Varieties of Nilpotent Matrices. III. Ann. of Math. 1959, 70 (1), 167-205.

[15] Gutiérrez Fernández, J.C. On Commutative Power-associative Nilalgebras. Comm. Algebra. 2004, 32 (6), 2243-2250.

[16] Gutiérrez Fernández, J.C.; Suazo, A. Commutative Power-associative Nilalgebras of Nilindex 5. Results Math. 2005, 47 (3-4), 296-304.

[17] Jacobson, N. Lie Algebras; Interscience: New York, 1962.

[18] Kuzmin, E.N.; Shestakov, I.P. Nonassociative structures, in: A.I. Kostrikin, I.R. Shafarevich (Eds.), Encyclopaedia of Mathematical Sciences, vol. 57, 197-280, 1994.

[19] Mathes, B.; Omladic, M.; Radjavi, H. Linear Spaces of Nilpotent Matrices. Linear Algebra Appl. 1991, 149, 215-225. 
[20] Schafer, R.D. Struture and Representation of Nonassociative Algebras; Source: Bull. Amer. Math. Soc. 1995, 61 (6), 469-484.

[21] Schafer, R.D. An introduction to Nonassociative Algebras; Academic Press: New York/London, 1966.

[22] Shestakov, I.P. Finite-dimensional Algebras with a Nil-basis. Algebra and Logic. 1971, 10, 58-65.

[23] Suttles, D. A Counterexample to a Conjecture of Albert. Notices Amer. Math. Soc. 1972, 19, A-566.

[24] Zelmanov, E.I. On Engel Lie Algebras. Siberian Math. J. 1988, 29 (5), 777-781.

[25] Zhevlakov, K.A; Slinko, A.M.; Shestakov, I.P; Shirshov, A.I. Rings that are Nearly Associative; Academic Press: New York/London, 1992. 


\section{Índice Remissivo}

A-Bimódulo, 7

Álgebra, 6

anulador de uma, 8

associativa, 6

comutativa, 6

de potências associativas, 10

Engel, 8

homomorfismo de, 8

Jordan, 8

não-associativa, 6

nilpotente, 9

quociente, 8

simples, 8

solúvel, 9

Índice

de nilpotência

de uma álgebra, 9

de uma transformação linear, 8

de solubilidade, 9

Bimódulo, 7 decomposição, 26

irredutível, 7, 24

subbimódulo, 7

Constantes de estrutura, 6

Ideal, 7

Identidade

de Jordan, 9

Nilálgebra, 10

Nilíndice, 10

Nilpotente

elemento, 10, 11

transformação linear, 8

Nilradical, 11

Operador

multiplicação à direita, 8

multiplicação à esquerda, 8

\section{Potências}

de um elemento, 9

de uma álgebra, 9 
Radical solúvel, 10

Soma direta, 8

Subálgebra, 7

Suttles

exemplo, 1 